\title{
Aryne-Mediated [2,3]-Sigmatropic Rearrangement of Tertiary Allylic Amines
}

Juan Zhang, Zhi-Xiong Chen, Ting Du, Bing Li, Yonghong Gu,* and Shi-Kai Tian*

Department of Chemistry, University of Science and Technology of China, Hefei, Anhui 230026, China

\section{Supporting Information}

\section{Table of Contents}

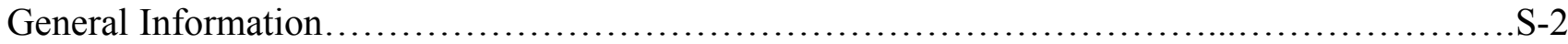

Preparation of Tertiary Allylic Amines....................................................

Optimization of the Reaction Conditions.................................................

General Procedure for the Aryne-Mediated [2,3]-Sigmatropic Rearrangement of Tertiary Allylic

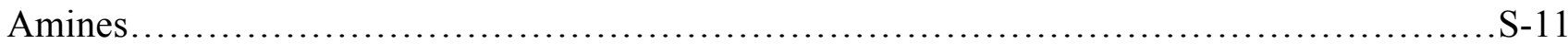

Analytical Data for the Products........................................................ 12

Chemical Transformations of the Products..................................................

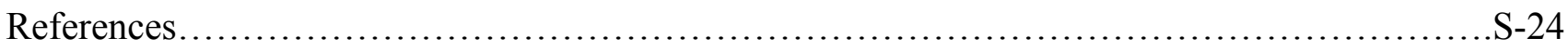

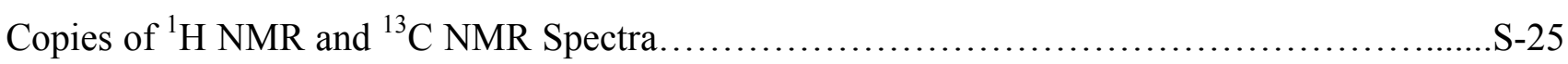

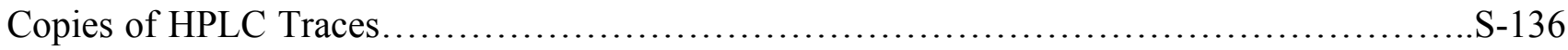

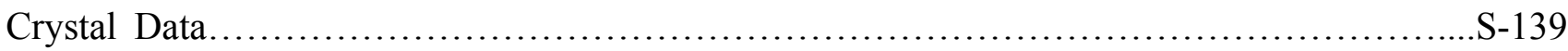




\section{General Information}

${ }^{1} \mathrm{H}$ NMR and ${ }^{13} \mathrm{C}$ NMR spectra were recorded on a Bruker AC-400 FT spectrometer $(400 \mathrm{MHz}$ and $100 \mathrm{MHz}$, respectively) using tetramethylsilane as an internal reference, and chemical shifts $(\delta)$ and coupling constants $(J)$ were expressed in ppm and $\mathrm{Hz}$, respectively. High resolution mass spectra (HRMS) were recorded on a LC-TOF spectrometer (Micromass). ESI-mass data were acquired using a Thermo LTQ Orbitrap XL instrument equipped with an ESI source and controlled by Xcalibur software. High pressure liquid chromatography (HPLC) analyses were performed on a Hewlett-Packard 1200 Series instrument equipped with anisostatic pump, using a Daicel Chiralpak column (OJ, OD, $250 \times 4.6 \mathrm{~mm}$ ) with isopropanol/hexane as mobile phase, and the UV detection was monitored at $254 \mathrm{~nm}$. Optical rotations were measured on a Perkin-Elmer 343 polarimeter with a sodium lamp at $\lambda=589 \mathrm{~nm}$ and reported as $[\alpha]_{\mathrm{D}}{ }^{\mathrm{T}}{ }^{\circ} \mathrm{C}(c=\mathrm{g} / 100 \mathrm{~mL}$, solvent $)$. Melting points were uncorrected.

Chemicals were purchased from the Energy chemical, Acros, Accela, Alfa Aesar, and TCI, and used as received. Amines $\mathbf{1 c},{ }^{1} \mathbf{1} \mathbf{p},{ }^{2}$ and $\mathbf{1} \mathbf{v}^{3}$ and 2-(trimethylsilyl)aryl triflates $\mathbf{2 a}-\mathbf{f}^{4}$ were prepared according to literature procedures.

Abbreviations: $\mathrm{DCE}=1$-2-dichloroethane, $\mathrm{DME}=1,2$-dimethoxyethane, $\mathrm{DMF}=N$, $N$-dimethylformamide, $\mathrm{DMSO}=$ dimethylsulfoxide, $\mathrm{EDCI}=1$-(3-dimethylaminopropyl)3-ethylcarbodiimide hydrochloride, $\mathrm{HOBt}=1$-hydroxybenzotriazole, $\mathrm{TBAF}=$ tetrabutylammonium fluoride, $\mathrm{Tf}=$ trifluoromethanesulfonyl, $\mathrm{THF}=$ tetrahydrofuran, $\mathrm{TMS}=$ trimethylsilyl.

\section{Preparation of Tertiary Allylic Amines}

(1) $N$-Alkylation of Secondary Amines with Alkyl Halides

(1.1) Preparation of Secondary Amines

2-Bromo-1-morpholinoethanone, ${ }^{5}$ N,2-dimethylallylamine, N,3-dimethylbut-2-en-1-amine, ${ }^{6}$ and $N$-bromoacetyl-( $1 S, 2 R)$-bornane-10,2-sultam ${ }^{7}$ were prepared according to literature procedures. The preparation of $(E)-N$-methyl-3-phenylprop-2-en-1-amine and $N$-methylcyclohex-2-enamine is shown below.

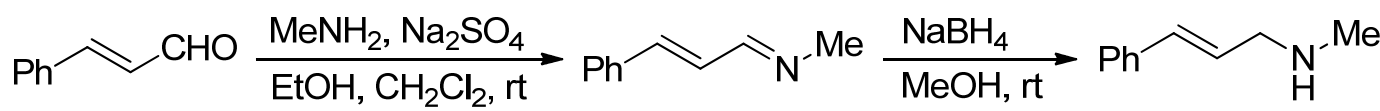

A mixture of cinnamaldehyde $(1.32 \mathrm{~g}, 10.0 \mathrm{mmol})$, methylamine $(1.65 \mathrm{~g}, 33 \% \mathrm{wt}$. in ethanol, $5.0 \mathrm{~mL}, 53.0 \mathrm{mmol})$, and sodium sulfate $(2.0 \mathrm{~g})$ in dichloromethane $(20 \mathrm{~mL})$ was stirred at room temperature for $14 \mathrm{~h}$. The mixture was filtered and concentrated. The residue was dissolved in methanol $(20 \mathrm{~mL})$ and added portionwise sodium borohydride $(454 \mathrm{mg}, 12.0 \mathrm{mmol})$ during $15 \mathrm{~min}$. The mixture was stirred at room temperature for $4 \mathrm{~h}$, concentrated, cooled with ice bath, and added slowly saturated aqueous ammonium chloride $(15 \mathrm{~mL})$. The residue was extracted with ethyl acetate $(3 \times 10 \mathrm{~mL})$, and to the organic layer was added hydrochloric acid $(1.0 \mathrm{M})$ until $\mathrm{pH}=2.0$. The aqueous layer was extracted with diethyl ether $(4 \times 10 \mathrm{~mL})$ and added aqueous sodium hydroxide $(1.0 \mathrm{M})$ until $\mathrm{pH}=13.0$. The mixture was extracted with ethyl acetate $(3 \times 10 \mathrm{~mL})$, and the organic layer was washed with brine, dried over anhydrous sodium sulfate, and concentrated to give crude (E)- $N$-methyl-3-phenylprop-2-en-1-amine as a colorless oil, which was subjected to the following $N$-alkylation reaction (see below). 


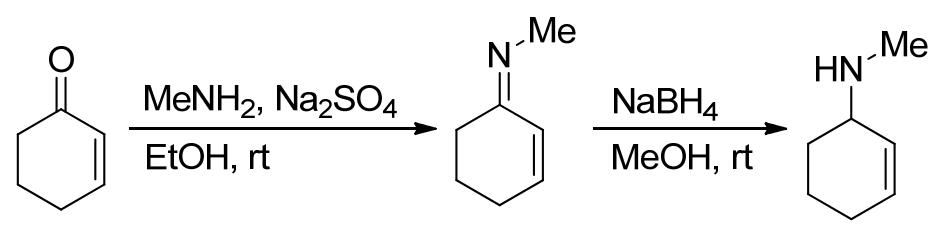

Crude $N$-methylcyclohex-2-enamine was prepared from cyclohex-2-enone according to the above procedure and subjected to the following $N$-alkylation reaction (see below).

(1.2) Condition A

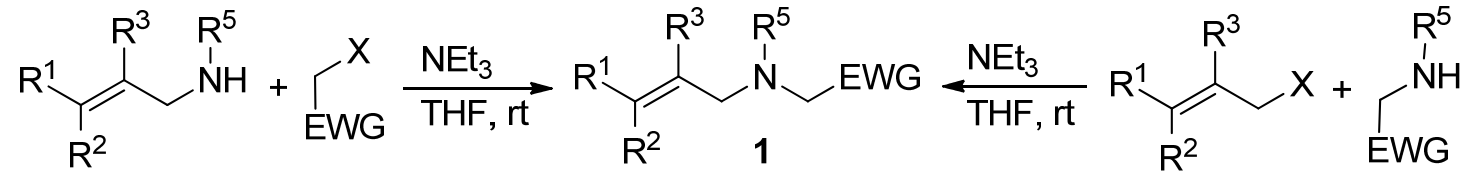

entry secondary amine $\quad$ alkyl halide $\quad \mathbf{1}$

1<smiles>C=CCNC(C)C</smiles>

$\mathrm{Br} \sim \mathrm{CO}_{2} \mathrm{Et}$<smiles>C=CCN(C)CCOOCC</smiles>

1a

2<smiles>C=CCNC</smiles><smiles>CC(=O)CCl</smiles><smiles>C=CCN(C)CC(C)=O</smiles>

1b

3<smiles>C=CCNC(C)C</smiles><smiles>NC(=O)CCl</smiles><smiles>C=CCN(C)CC(N)=O</smiles>
1d

4<smiles>C=CCNC</smiles><smiles>CNC(=O)CBr</smiles><smiles>C=CCN(C)CC(=O)NC</smiles>

5<smiles>C=CCNC(C)C</smiles><smiles>O=C(CBr)N1CCOCC1</smiles><smiles>C=CCN(C)CC(=O)N1CCOCC1</smiles>

\section{$1 f$}

6<smiles>C=CCNC(C)C</smiles>

$\mathrm{Br} \smile \mathrm{CN}$<smiles>C=CCN(C)CC#N</smiles>

$1 \mathrm{~g}$

7<smiles>C=CCNC(C)C</smiles><smiles>ClCc1nc2ccccc2o1</smiles>

8<smiles>C=C(C)CNC</smiles>

$\mathrm{Br} \sim \mathrm{CO}_{2} \mathrm{Et}$<smiles>C=CCN(C)Cc1nc2ccccc2o1</smiles><smiles>C=C(C)CN(C)COOCC</smiles>

1k 
9<smiles>CNCC=C(C)C</smiles>

10<smiles>CNC/C=C/c1ccccc1</smiles>

11<smiles>CNC1C=CCCC1</smiles>

12<smiles>C=CCNCCC</smiles>

13<smiles>C#CCNCC=C</smiles>

14<smiles>C=CCN</smiles><smiles>O=C1CNCCN1</smiles>

16<smiles>C=CCNCC=C</smiles><smiles>CCOC(=O)CBr</smiles>

$\mathrm{Br} \sim \mathrm{CO}_{2} \mathrm{Et}$<smiles>CCOC(=O)CBr</smiles><smiles>CCOC(=O)CBr</smiles><smiles>CCOC(=O)CBr</smiles><smiles>CCOC(=O)CBr</smiles><smiles>C=CCBr</smiles><smiles>CC12CCCC(C(=O)N(C(=O)CBr)C1=O)C2(C)C</smiles><smiles>CCOC(=O)CN(C)CC=C(C)C</smiles><smiles>CCOC(=O)CN(C)C/C=C/c1ccccc1</smiles><smiles>CCOC(=O)CN(C)C1C=CCCC1</smiles>

$1 n$<smiles>C=CCN(CCC)CC(=O)OCC</smiles>

10<smiles>C#CCN(CC=C)CC(=O)OCC</smiles>

19<smiles>C=CCN(CCOCC)CCOC(=O)OCC</smiles>

$1 \mathrm{r}$<smiles>C=CCN1CCNC(=O)C1</smiles>

1t<smiles>C=CCN(CC=C)CC(=O)N1CC2CCC(C)(C1)C2(C)C</smiles>

To a solution of the secondary amine $(5.0 \mathrm{mmol})$, triethylamine $(556 \mathrm{mg}, 0.76 \mathrm{~mL}, 5.5 \mathrm{mmol})$ in tetrahydrofuran $(10 \mathrm{~mL})$ at room temperature was added the alkyl halide $(5.0 \mathrm{mmol})$. The mixture was stirred for $14 \mathrm{~h}$, filtered, and concentrated.

For the preparation of amines $\mathbf{1 b}, \mathbf{1 d}-\mathbf{g}, \mathbf{1 i}$, and $\mathbf{1 t}$ : The resulting residue was acidized with hydrochloric acid $(6.0 \mathrm{M})$ until $\mathrm{pH}=2.0$ and extracted with diethyl ether $(4 \times 10 \mathrm{~mL})$. The aqueous acidic layer was treated with aqueous sodium hydroxide $(1.0 \mathrm{M})$ until $\mathrm{pH}=13.0$ and extracted with ethyl acetate $(3 \times 10 \mathrm{~mL})$. The organic layer was washed with brine, dried over anhydrous sodium sulfate, and concentrated to give the tertiary allylic amine. 
For the preparation of amines 1a, 1k-o, 1q, 1r, and 1x: The resulting residue was subjected to silica gel chromatography, using a mixture of ethyl acetate and petroleum ether $(1: 30 \sim 1: 4)$ as the eluent, to give the tertiary allylic amine.

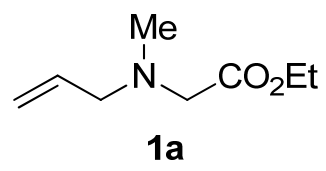

Ethyl 2-(allyl(methyl)amino)acetate (1a) was obtained (628 $\mathrm{mg}, 80 \%$ yield) as a pale yellow oil. ${ }^{1} \mathrm{H}$ NMR (400 MHz, $\left.\mathrm{CDCl}_{3}\right) \delta 5.94-5.82(\mathrm{~m}, 1 \mathrm{H}), 5.24-5.13(\mathrm{~m}, 2 \mathrm{H}), 4.19$ (q, $\left.J=7.2 \mathrm{~Hz}, 2 \mathrm{H}\right), 3.23$ $(\mathrm{s}, 2 \mathrm{H}), 3.14(\mathrm{~d}, J=6.4 \mathrm{~Hz}, 2 \mathrm{H}), 2.37(\mathrm{~s}, 3 \mathrm{H}), 1.28(\mathrm{t}, J=7.2 \mathrm{~Hz}, 3 \mathrm{H}) ;{ }^{13} \mathrm{C} \mathrm{NMR}\left(100 \mathrm{MHz}, \mathrm{CDCl}_{3}\right)$ $\delta 171.0,135.2,118.3,60.5,60.4,57.7,42.5,14.3$; HRMS (ESI) calcd for $\mathrm{C}_{8} \mathrm{H}_{16} \mathrm{NO}_{2}{ }^{+}(\mathrm{M}+\mathrm{H})^{+}$ 158.1176 , found 158.1175 .

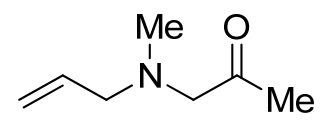

$1 \mathrm{~b}$

1-(Allyl(methyl)amino)propan-2-one (1b) ${ }^{2}$ was obtained $(318 \mathrm{mg}, 50 \%$ yield) as a yellow oil. ${ }^{1} \mathrm{H}$ NMR $\left(400 \mathrm{MHz}, \mathrm{CDCl}_{3}\right) \delta 5.92-5.81(\mathrm{~m}, 1 \mathrm{H}), 5.22-5.13(\mathrm{~m}, 2 \mathrm{H}), 3.19(\mathrm{~s}, 2 \mathrm{H}), 3.06(\mathrm{dt}, J=6.8$, $1.2 \mathrm{~Hz}, 2 \mathrm{H}), 2.28$ (s, 3H), 2.15 (s, 3H); ${ }^{13} \mathrm{C} \mathrm{NMR}\left(100 \mathrm{MHz}, \mathrm{CDCl}_{3}\right) \delta 207.5,135.2,118.3,66.6$, 60.9, 42.9, 27.7; HRMS (ESI) calcd for $\mathrm{C}_{7} \mathrm{H}_{14} \mathrm{NO}^{+}(\mathrm{M}+\mathrm{H})^{+}$128.1070, found 128.1068.

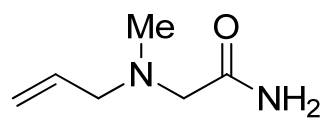

$1 d$

2-(Allyl(methyl)amino)acetamide (1d) was obtained (256 mg, 40\% yield) as a white solid. m.p. 45-47 ${ }^{\circ} \mathrm{C} ;{ }^{1} \mathrm{H}$ NMR $\left(400 \mathrm{MHz}, \mathrm{CDCl}_{3}\right) \delta 7.06(\mathrm{~s}, \mathrm{br}, 1 \mathrm{H}), 6.00(\mathrm{~s}, \mathrm{br}, 1 \mathrm{H}), 5.88-5.76(\mathrm{~m}, 1 \mathrm{H})$, 5.24-5.15 (m, 2H), $3.08(\mathrm{~d}, J=6.4 \mathrm{~Hz}, 2 \mathrm{H}), 3.01(\mathrm{~s}, 2 \mathrm{H}), 2.31(\mathrm{~s}, 3 \mathrm{H}) ;{ }^{13} \mathrm{C} \mathrm{NMR}\left(100 \mathrm{MHz}, \mathrm{CDCl}_{3}\right)$ $\delta$ 174.3, 134.8, 118.5, 61.1, 60.4, 43.3; HRMS (ESI) calcd for $\mathrm{C}_{6} \mathrm{H}_{13} \mathrm{~N}_{2} \mathrm{O}^{+}(\mathrm{M}+\mathrm{H})^{+} 129.1022$, found 129.1021.

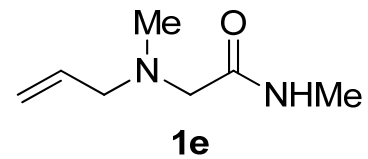

2-(Allyl(methyl)amino)- $N$-methylacetamide (1e) was obtained (320 $\mathrm{mg}, 45 \%$ yield) as a yellow oil. ${ }^{1} \mathrm{H}$ NMR $\left(400 \mathrm{MHz}, \mathrm{CDCl}_{3}\right) \delta 7.19(\mathrm{~s}, \mathrm{br}, 1 \mathrm{H}), 5.87-5.75(\mathrm{~m}, 1 \mathrm{H}), 5.24-5.15(\mathrm{~m}, 2 \mathrm{H}), 3.06(\mathrm{~d}, J$ $=6.4 \mathrm{~Hz}, 2 \mathrm{H}), 3.02(\mathrm{~s}, 2 \mathrm{H}), 2.85(\mathrm{~d}, J=4.8 \mathrm{~Hz}, 3 \mathrm{H}), 2.28(\mathrm{~s}, 3 \mathrm{H}) ;{ }^{13} \mathrm{C} \mathrm{NMR}\left(100 \mathrm{MHz}, \mathrm{CDCl}_{3}\right) \delta$ 171.6, 134.8, 118.4, 61.0, 60.4, 43.3, 25.8; HRMS (ESI) calcd for $\mathrm{C}_{7} \mathrm{H}_{15} \mathrm{~N}_{2} \mathrm{O}^{+}(\mathrm{M}+\mathrm{H})^{+} 143.1179$, found 143.1178 . 


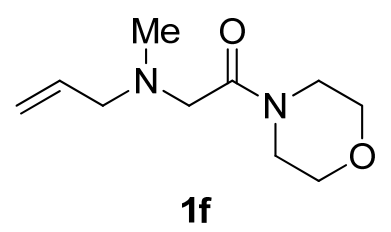

2-(Allyl(methyl)amino)-1-morpholinoethanone (1f) was obtained (446 $\mathrm{mg}, 45 \%$ yield) as a yellow oil. ${ }^{1} \mathrm{H}$ NMR $\left(400 \mathrm{MHz}, \mathrm{CDCl}_{3}\right) \delta$ 5.89-5.77 (m, $\left.1 \mathrm{H}\right), 5.23-5.14(\mathrm{~m}, 2 \mathrm{H}), 3.70-3.58(\mathrm{~m}, 8 \mathrm{H})$, $3.17(\mathrm{~s}, 2 \mathrm{H}), 3.04(\mathrm{~d}, J=6.0 \mathrm{~Hz}, 2 \mathrm{H}), 2.27(\mathrm{~s}, 3 \mathrm{H}) ;{ }^{13} \mathrm{C} \mathrm{NMR}\left(100 \mathrm{MHz}, \mathrm{CDCl}_{3}\right) \delta 168.9,134.9$, 118.2, 66.9, 60.6, 60.0, 46.1, 42.3, 42.1; HRMS (ESI) calcd for $\mathrm{C}_{10} \mathrm{H}_{19} \mathrm{~N}_{2} \mathrm{O}_{2}{ }^{+}(\mathrm{M}+\mathrm{H})^{+}$199.1441, found 199.1440 .

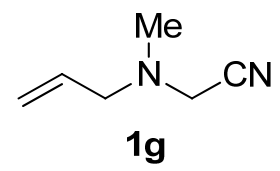

2-(Allyl(methyl)amino)acetonitrile (1g) was obtained (386 mg, 70\% yield) as a colorless oil. ${ }^{1} \mathrm{H}$ NMR (400 MHz, $\left.\mathrm{CDCl}_{3}\right) \delta$ 5.84-5.72 (m, 1H), 5.34-5.20 (m, 2H), $3.54(\mathrm{~s}, 2 \mathrm{H}), 3.10(\mathrm{~d}, J=6.4 \mathrm{~Hz}$, 2H), 2.39 (s, 3H); $\left.{ }^{13} \mathrm{C} \mathrm{NMR} \mathrm{(100} \mathrm{MHz,} \mathrm{CDCl}_{3}\right) \delta 134.0,119.5,114.6,58.9,44.2,42.1$; HRMS (ESI) calcd for $\mathrm{C}_{6} \mathrm{H}_{11} \mathrm{~N}_{2}^{+}(\mathrm{M}+\mathrm{H})^{+}$111.0917, found 111.0916.

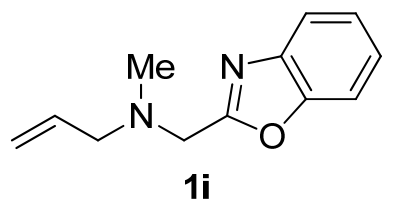

$N$-(Benzo $[d]$ oxazol-2-ylmethyl)- $N$-methylprop-2-en-1-amine (1i) was obtained $(607 \mathrm{mg}, 60 \%$ yield) as an orange oil. ${ }^{1} \mathrm{H}$ NMR $\left(400 \mathrm{MHz} \mathrm{CDCl}_{3}\right) \delta 7.75-7.69(\mathrm{~m}, 1 \mathrm{H}), 7.56-7.51(\mathrm{~m}, 1 \mathrm{H})$, 7.37-7.30 (m, 2H), 6.00-5.88 (m, 1H), 5.30-5.18 (m, 2H), $3.89(\mathrm{~s}, 2 \mathrm{H}), 3.21(\mathrm{~d}, J=6.8 \mathrm{~Hz}, 2 \mathrm{H}), 2.40$ $(\mathrm{s}, 3 \mathrm{H}) ;{ }^{13} \mathrm{C} \mathrm{NMR}\left(100 \mathrm{MHz}, \mathrm{CDCl}_{3}\right) \delta 163.9,151.1,141.1,134.9,125.1,124.4,120.1,118.8,110.8$, 60.6, 53.7, 42.5; HRMS (ESI) calcd for $\mathrm{C}_{12} \mathrm{H}_{15} \mathrm{~N}_{2} \mathrm{O}^{+}(\mathrm{M}+\mathrm{H})^{+}$203.1179, found 203.1180.<smiles>C=C(C)CN(C)CCOCC</smiles>

$1 \mathrm{k}$

Ethyl 2-(methyl(2-methylallyl)amino)acetate (1k) was obtained (385 $\mathrm{mg}, 45 \%$ yield) as a colorless oil. ${ }^{1} \mathrm{H}$ NMR (400 MHz, $\left.\mathrm{CDCl}_{3}\right) \delta 4.91-4.85(\mathrm{~m}, 2 \mathrm{H}), 4.18(\mathrm{q}, J=7.2 \mathrm{~Hz}, 2 \mathrm{H}), 3.21$ (s, 2H), $3.03(\mathrm{~s}, 2 \mathrm{H}), 2.35(\mathrm{~s}, 3 \mathrm{H}), 1.77(\mathrm{~s}, 3 \mathrm{H}), 1.28(\mathrm{t}, J=7.2 \mathrm{~Hz}, 3 \mathrm{H}) ;{ }^{13} \mathrm{C} \mathrm{NMR}\left(100 \mathrm{MHz}, \mathrm{CDCl}_{3}\right) \delta$ 171.2, 143.1, 113.6, 64.0, 60.4, 57.8, 42.6, 20.7, 14.4; HRMS (ESI) calcd for $\mathrm{C}_{9} \mathrm{H}_{18} \mathrm{NO}_{2}{ }^{+}(\mathrm{M}+\mathrm{H})^{+}$ 172.1332 , found 172.1332 .<smiles>CCOC(=O)CN(C)CC=C(C)C</smiles> 
Ethyl 2-(methyl(3-methylbut-2-en-1-yl)amino)acetate (11) was obtained (556 $\mathrm{mg}, 60 \%$ yield) as a yellow oil. ${ }^{1} \mathrm{H}$ NMR (400 MHz, $\left.\mathrm{CDCl}_{3}\right) \delta 5.26(\mathrm{t}, J=7.2 \mathrm{~Hz}, 1 \mathrm{H}), 4.19$ (q, $\left.J=7.2 \mathrm{~Hz}, 2 \mathrm{H}\right), 3.21(\mathrm{~s}$, 2H), $3.09(\mathrm{~d}, J=7.2 \mathrm{~Hz}, 2 \mathrm{H}), 2.35(\mathrm{~s}, 3 \mathrm{H}), 1.74(\mathrm{~s}, 3 \mathrm{H}), 1.65(\mathrm{~s}, 3 \mathrm{H}), 1.28(\mathrm{t}, J=7.2 \mathrm{~Hz}, 3 \mathrm{H}) ;{ }^{13} \mathrm{C}$ NMR $\left(100 \mathrm{MHz}, \mathrm{CDCl}_{3}\right) \delta 171.2,136.0,121.1,60.5,57.8,54.7,42.6,26.0,18.0,14.4$; HRMS (ESI) calcd for $\mathrm{C}_{10} \mathrm{H}_{20} \mathrm{NO}_{2}^{+}(\mathrm{M}+\mathrm{H})^{+}$186.1489, found 186.1487 .

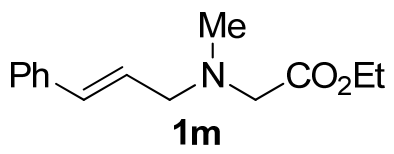

Ethyl 2-(cinnamyl(methyl)amino)acetate (1m) was obtained [816 $\mathrm{mg}, 35 \%$ yield (3 steps from cinnamaldehyde)] as a yellow oil. ${ }^{1} \mathrm{H}$ NMR $\left(400 \mathrm{MHz}, \mathrm{CDCl}_{3}\right) \delta 7.41-7.20(\mathrm{~m}, 5 \mathrm{H}), 6.53(\mathrm{~d}, J=16.0$ $\mathrm{Hz}, 1 \mathrm{H}), 6.29$ (dt, $J=16.0,6.8 \mathrm{~Hz}, 1 \mathrm{H}), 4.18$ (q, $J=7.2 \mathrm{~Hz}, 2 \mathrm{H}), 3.33-3.25(\mathrm{~m}, 4 \mathrm{H}), 2.42(\mathrm{~s}, 3 \mathrm{H})$, $1.26(\mathrm{t}, J=7.2 \mathrm{~Hz}, 3 \mathrm{H}) ;{ }^{13} \mathrm{C}$ NMR $\left(100 \mathrm{MHz}, \mathrm{CDCl}_{3}\right) \delta 171.0,136.9,133.3,128.7,127.7,126.8$, 126.4, 60.6, 59.8, 57.9, 42.7, 14.4; HRMS (ESI) calcd for $\mathrm{C}_{14} \mathrm{H}_{20} \mathrm{NO}_{2}^{+}(\mathrm{M}+\mathrm{H})^{+} 234.1489$, found 234.1487.<smiles>CCOC(=O)CN(C)C1C=CCCC1</smiles>

1n

Ethyl 2-(cyclohex-2-en-1-yl(methyl)amino)acetate (1n) was obtained [395 mg, 20\% yield (3 steps from cyclohex-2-enone)] as a yellow oil. ${ }^{1} \mathrm{H} \mathrm{NMR}\left(400 \mathrm{MHz}, \mathrm{CDCl}_{3}\right) \delta 5.88-5.81(\mathrm{~m}, 1 \mathrm{H})$, 5.68-5.62 (m, 1H), 4.19 (q, $J=7.2 \mathrm{~Hz}, 2 \mathrm{H}), 3.41-3.31(\mathrm{~m}, 2 \mathrm{H}), 3.18(\mathrm{~d}, J=16.4 \mathrm{~Hz}, 1 \mathrm{H}), 2.40$ (s, $3 \mathrm{H}), 1.94-2.01(\mathrm{~m}, 2 \mathrm{H}), 1.87-1.75(\mathrm{~m}, 2 \mathrm{H}), 1.60-1.36(\mathrm{~m}, 2 \mathrm{H}), 1.27(\mathrm{t}, J=7.2 \mathrm{~Hz}, 3 \mathrm{H}) ;{ }^{13} \mathrm{C}$ NMR $\left(100 \mathrm{MHz}, \mathrm{CDCl}_{3}\right) \delta 171.9,130.9,129.4,60.7,59.9,54.6,39.3,25.3,23.2,21.5,14.4$; HRMS (ESI) calcd for $\mathrm{C}_{11} \mathrm{H}_{20} \mathrm{NO}_{2}^{+}(\mathrm{M}+\mathrm{H})^{+}$198.1489, found 198.1487.

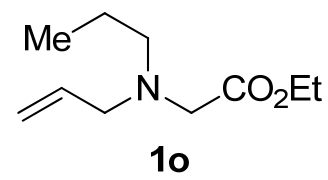

Ethyl 2-(allyl(propyl)amino)acetate (10) was obtained (371 mg, 40\% yield) as a yellow oil. ${ }^{1} \mathrm{H}$ NMR (400 MHz, $\left.\mathrm{CDCl}_{3}\right) \delta 5.93-5.81(\mathrm{~m}, 1 \mathrm{H}), 5.23-5.11(\mathrm{~m}, 2 \mathrm{H}), 4.17(\mathrm{q}, J=7.2 \mathrm{~Hz}, 2 \mathrm{H}), 3.32(\mathrm{~s}$, $2 \mathrm{H}), 3.25(\mathrm{~d}, J=6.4 \mathrm{~Hz}, 2 \mathrm{H}), 2.59-2.51(\mathrm{~m}, 2 \mathrm{H}), 1.55-1.44(\mathrm{~m}, 2 \mathrm{H}), 1.27(\mathrm{t}, J=7.2 \mathrm{~Hz}, 3 \mathrm{H}), 0.89(\mathrm{t}$, $J=7.2 \mathrm{~Hz}, 3 \mathrm{H}) ;{ }^{13} \mathrm{C} \mathrm{NMR}\left(100 \mathrm{MHz}, \mathrm{CDCl}_{3}\right) \delta 171.7,135.7,117.9,60.4,57.7,56.3,54.7,20.7$, 14.4, 11.9; HRMS (ESI) calcd for $\mathrm{C}_{10} \mathrm{H}_{20} \mathrm{NO}_{2}{ }^{+}(\mathrm{M}+\mathrm{H})^{+}$186.1489, found 186.1488.

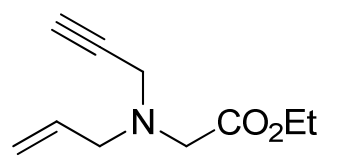

1q 
Ethyl 2-(allyl(prop-2-yn-1-yl)amino)acetate (1q) was obtained (453 $\mathrm{mg}, 50 \%$ yield) as a colorless oil. ${ }^{1} \mathrm{H}$ NMR (400 MHz, $\left.\mathrm{CDCl}_{3}\right) \delta 5.94-5.82(\mathrm{~m}, 1 \mathrm{H}), 5.30-5.17(\mathrm{~m}, 2 \mathrm{H}), 4.19$ (q, $J=7.2$ $\mathrm{Hz}, 2 \mathrm{H}), 3.57(\mathrm{~d}, J=2.4 \mathrm{~Hz}, 2 \mathrm{H}), 3.39(\mathrm{~s}, 2 \mathrm{H}), 3.24(\mathrm{~d}, J=6.8 \mathrm{~Hz}, 2 \mathrm{H}), 2.24(\mathrm{t}, J=2.4 \mathrm{~Hz}, 1 \mathrm{H})$, $1.28(\mathrm{t}, J=7.2 \mathrm{~Hz}, 3 \mathrm{H}) ;{ }^{13} \mathrm{C} \mathrm{NMR}\left(100 \mathrm{MHz}, \mathrm{CDCl}_{3}\right) \delta 170.9,134.9,119.0,78.2,73.7,60.8,57.2$, 53.9, 42.6, 14.3; HRMS (ESI) calcd for $\mathrm{C}_{10} \mathrm{H}_{16} \mathrm{NO}_{2}{ }^{+}(\mathrm{M}+\mathrm{H})^{+}$182.1776, found 182.1776.

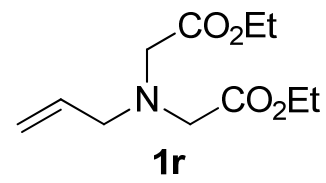

Diethyl 2,2'-(allylazanediyl)diacetate (1r) was obtained (344 $\mathrm{mg}, 30 \%$ yield) as a pale yellow oil. ${ }^{1} \mathrm{H}$ NMR (400 MHz, $\left.\mathrm{CDCl}_{3}\right) \delta 5.94-5.83(\mathrm{~m}, 1 \mathrm{H}), 5.26-5.14(\mathrm{~m}, 2 \mathrm{H}), 4.17(\mathrm{q}, J=7.2 \mathrm{~Hz}, 4 \mathrm{H})$, $3.56(\mathrm{~s}, 4 \mathrm{H}), 3.39(\mathrm{~d}, J=6.4 \mathrm{~Hz}, 2 \mathrm{H}), 1.27(\mathrm{t}, J=7.2 \mathrm{~Hz}, 6 \mathrm{H}) ;{ }^{13} \mathrm{C} \mathrm{NMR}\left(100 \mathrm{MHz}, \mathrm{CDCl}_{3}\right) \delta 171.2$, 135.2, 118.8, 60.6, 57.6, 54.4, 14.4; HRMS (ESI) calcd for $\mathrm{C}_{11} \mathrm{H}_{20} \mathrm{NO}_{4}{ }^{+}(\mathrm{M}+\mathrm{H})^{+} 230.1387$, found 230.1386 .

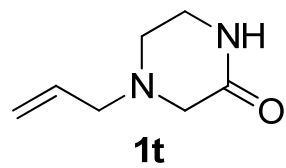

4-Allylpiperazin-2-one (1t) was obtained (456 mg, 65\% yield) as a white solid. m.p. $77-79{ }^{\circ} \mathrm{C}$; ${ }^{1} \mathrm{H}$ NMR $\left(400 \mathrm{MHz}, \mathrm{CDCl}_{3}\right) \delta 7.18(\mathrm{~s}, \mathrm{br}, 1 \mathrm{H}), 5.89-5.77(\mathrm{~m}, 1 \mathrm{H})$, 5.27-5.18 (m, 2H), 3.39-3.33 (m, $2 \mathrm{H}), 3.13(\mathrm{~s}, 2 \mathrm{H}), 3.07(\mathrm{dd}, J=6.4,0.8 \mathrm{~Hz}, 2 \mathrm{H}), 2.65(\mathrm{t}, J=5.2 \mathrm{~Hz}, 2 \mathrm{H}) ;{ }^{13} \mathrm{C} \mathrm{NMR}(100 \mathrm{MHz}$, $\left.\mathrm{CDCl}_{3}\right) \delta 170.0,133.9,118.9,60.5,56.8,48.6,41.3$; HRMS (ESI) calcd for $\mathrm{C}_{7} \mathrm{H}_{13} \mathrm{~N}_{2} \mathrm{O}^{+}(\mathrm{M}+\mathrm{H})^{+}$ 141.1022, found 141.1022 .

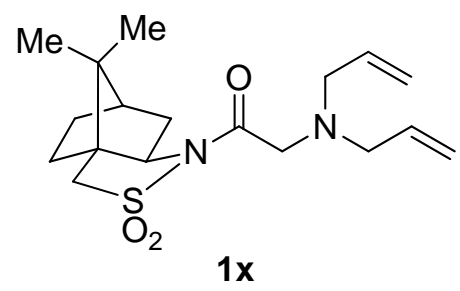

2-(Diallylamino)-1-((6R,7a $R)-8,8$-dimethyl-2,2-dioxidohexahydro-1H-3a,6-methanobenzo[c]iso thiazol-1-yl)ethanone $(\mathbf{1 x})$ was obtained $(1.14 \mathrm{~g}, 65 \%$ yield $)$ as a white solid. m.p. $69-71{ }^{\circ} \mathrm{C} ;[\alpha]_{\mathrm{D}}{ }^{20}=$ -80.5 ( $c=0.80$, EtOAc); ${ }^{1} \mathrm{H}$ NMR (400 MHz, $\left.\mathrm{CDCl}_{3}\right) \delta 5.92-5.80(\mathrm{~m}, 2 \mathrm{H}), 5.23-5.10(\mathrm{~m}, 4 \mathrm{H}), 3.88$ $(\mathrm{dd}, J=7.2,5.6 \mathrm{~Hz}, 1 \mathrm{H}), 3.78-3.66(\mathrm{~m}, 2 \mathrm{H}), 3.48(\mathrm{~d}, J=13.6 \mathrm{~Hz}, 1 \mathrm{H}), 3.41(\mathrm{~d}, J=13.6 \mathrm{~Hz}, 1 \mathrm{H})$, 3.35-3.21 (m, 4H), 2.17-2.03 (m, 2H), 1.97-1.82 (m, 3H), 1.45-1.30 (m, 2H), $1.13(\mathrm{~s}, 3 \mathrm{H}), 0.97$ (s, $3 \mathrm{H}) ;{ }^{13} \mathrm{C} \mathrm{NMR}\left(100 \mathrm{MHz}, \mathrm{CDCl}_{3}\right) \delta 170.4,135.4,118.2,65.2,57.3,54.9,53.0,48.9,47.8,44.7,38.5$, 32.9, 26.5, 20.9, 20.0; HRMS (ESI) calcd for $\mathrm{C}_{18} \mathrm{H}_{29} \mathrm{~N}_{2} \mathrm{O}_{3} \mathrm{~S}^{+}(\mathrm{M}+\mathrm{H})^{+}$353.1893, found 353.1894.

(1.3) Condition B 


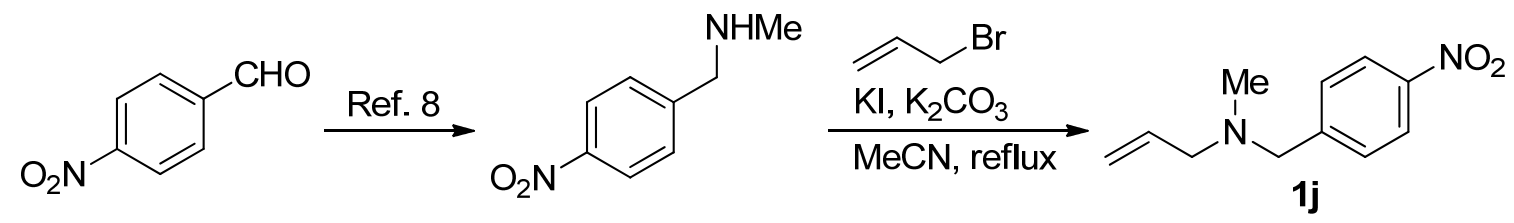

To a solution of $N$-methyl-1-(4-nitrophenyl)methanamine ${ }^{8}(665 \mathrm{mg}, 4.0 \mathrm{mmol})$ in acetonitrile $(10 \mathrm{~mL})$ under nitrogen was added allyl bromide $(532 \mathrm{mg}, 0.38 \mathrm{~mL}, 4.4 \mathrm{mmol})$, potassium carbonate (663 mg, $4.8 \mathrm{mmol}$ ), and potassium iodide $(133 \mathrm{mg}, 0.80 \mathrm{mmol}$ ). The mixture was heated to reflux for $14 \mathrm{~h}$, cooled to room temperature, and added water $(15 \mathrm{~mL})$. The mixture was extracted with ethyl acetate $(3 \times 10 \mathrm{~mL})$, washed with brine, dried over anhydrous sodium sulfate, and concentrated. The residue was subjected to silica gel chromatography, using a mixture of ethyl acetate and petroleum ether (1:10) as the eluent, to give $N$-methyl- $N$-(4-nitrobenzyl)prop-2-en-1-amine (1j) (247 $\mathrm{mg}, 30 \%$ yield) as a yellow oil. ${ }^{1} \mathrm{H}$ NMR $\left(400 \mathrm{MHz}, \mathrm{CDCl}_{3}\right) \delta 8.20-8.15(\mathrm{~m}, 2 \mathrm{H}), 7.51(\mathrm{~d}, J=8.8 \mathrm{~Hz}$, $2 \mathrm{H}), 5.95-5.84(\mathrm{~m}, 1 \mathrm{H}), 5.26-5.16(\mathrm{~m}, 2 \mathrm{H}), 3.58(\mathrm{~s}, 2 \mathrm{H}), 3.05(\mathrm{~d}, J=6.4 \mathrm{~Hz}, 2 \mathrm{H}), 2.21(\mathrm{~s}, 3 \mathrm{H}) ;{ }^{13} \mathrm{C}$ NMR (100 MHz, $\left.\mathrm{CDCl}_{3}\right) \delta 147.4,147.2,135.4,129.5,123.6,118.1,60.9,60.8,42.4$; HRMS (ESI) calcd for $\mathrm{C}_{11} \mathrm{H}_{15} \mathrm{~N}_{2} \mathrm{O}_{2}^{+}(\mathrm{M}+\mathrm{H})^{+} 207.1128$, found 207.1129.

(1.4) Condition C

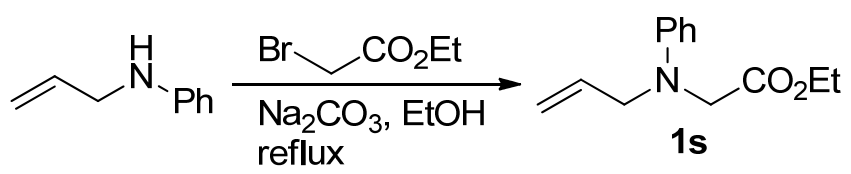

To a mixture of $N$-allylaniline $(599 \mathrm{mg}, 0.61 \mathrm{~mL}, 4.5 \mathrm{mmol})$ and sodium carbonate $(572 \mathrm{mg}, 5.4$ $\mathrm{mmol})$ in ethanol $(20 \mathrm{~mL})$ was added ethyl bromoacetate $(751 \mathrm{mg}, 0.50 \mathrm{~mL}, 4.5 \mathrm{mmol})$. The mixture was heated to reflux for $16 \mathrm{~h}$, cooled to room temperature, filtered, and concentrated. The residue was subjected to silica gel chromatography, using a mixture of ethyl acetate and petroleum ether (1:100) as the eluent, to give ethyl 2-(allyl(phenyl)amino)acetate (1s) (789 $\mathrm{mg}, 80 \%$ yield) as a yellow oil. ${ }^{1} \mathrm{H}$ NMR $\left(400 \mathrm{MHz}, \mathrm{CDCl}_{3}\right) \delta$ 7.24-7.18 (m, 2H), 6.76-6.71 (m, 1H), 6.69-6.64 (m, 2H), 5.95-5.84 (m, 1H), 5.27-5.16 (m, 2H), $4.20(\mathrm{q}, J=7.2 \mathrm{~Hz}, 2 \mathrm{H}), 4.05-4.00(\mathrm{~m}, 4 \mathrm{H}), 1.26(\mathrm{t}, J=7.2 \mathrm{~Hz}$, $3 \mathrm{H}) ;{ }^{13} \mathrm{C}$ NMR $\left(100 \mathrm{MHz}, \mathrm{CDCl}_{3}\right) \delta 171.4,148.4,134.0,129.3,117.5,116.6,112.5,61.1,54.4,52.3$, 14.4; HRMS (ESI) calcd for $\mathrm{C}_{13} \mathrm{H}_{18} \mathrm{NO}_{2}^{+}(\mathrm{M}+\mathrm{H})^{+}$220.1332, found 220.1332.

\section{(1.5) Condition D}

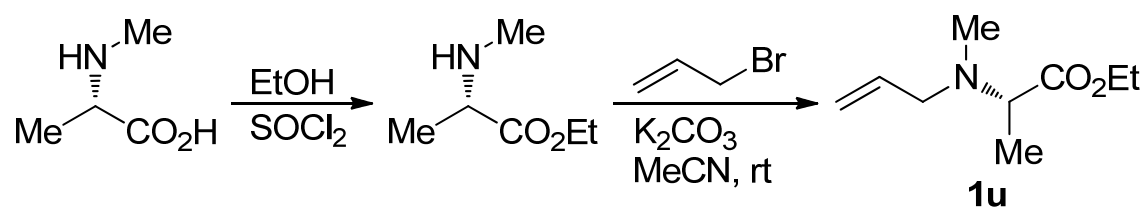

To a solution of $N$-methyl- $L$-alanine $(516 \mathrm{mg}, 5.0 \mathrm{mmol})$ in ethanol $(10 \mathrm{~mL})$ at $0{ }^{\circ} \mathrm{C}$ was added dropwise thionyl chloride $(1.78 \mathrm{~g}, 1.1 \mathrm{~mL}, 15.0 \mathrm{mmol})$. The mixture was heated at $40{ }^{\circ} \mathrm{C}$ for $5 \mathrm{~h}$ and concentrated. The resulting crude $N$-methyl- $L$-alanine ethyl ester was dissolved in acetonitrile (20 $\mathrm{mL})$ and added potassium carbonate $(1.70 \mathrm{~g}, 12.3 \mathrm{mmol})$ and allylbromide $(762 \mathrm{mg}, 0.55 \mathrm{~mL}, 6.3$ $\mathrm{mmol})$. The mixture was stirred at room temperature for $24 \mathrm{~h}$ and poured into saturated sodium 
bicarbonate. The mixture was extracted with dichloromethane $(3 \times 10 \mathrm{~mL})$, dried over anhydrous sodium sulfate, and concentrated. The residue was subjected to silica gel chromatography, using a mixture of ethyl acetate and petroleum ether (1:30) as the eluent, to give (S)-ethyl 2-(allyl(methyl)amino)propanoate (1u) $\left(514 \mathrm{mg}, 60 \%\right.$ yield) as a pale yellow oil. $[\alpha]_{\mathrm{D}}{ }^{20}=-55.8(c=$ 1.2, EtOAc); ${ }^{1} \mathrm{H}$ NMR $\left(400 \mathrm{MHz}, \mathrm{CDCl}_{3}\right) \delta 5.90-5.78(\mathrm{~m}, 1 \mathrm{H}), 5.23-5.11(\mathrm{~m}, 2 \mathrm{H}), 4.22-4.14(\mathrm{~m}, 2 \mathrm{H})$, $3.43(\mathrm{q}, J=6.8 \mathrm{~Hz}, 1 \mathrm{H}), 3.23-3.10(\mathrm{~m}, 2 \mathrm{H}), 2.31(\mathrm{~s}, 3 \mathrm{H}), 1.31-1.26(\mathrm{~m}, 6 \mathrm{H}) ;{ }^{13} \mathrm{C} \mathrm{NMR}(100 \mathrm{MHz}$, $\left.\mathrm{CDCl}_{3}\right) \delta 173.5,136.1,117.6,60.8,60.3,57.7,37.8,14.8,14.5$; HRMS (ESI) calcd for $\mathrm{C}_{9} \mathrm{H}_{18} \mathrm{NO}_{2}{ }^{+}$ $(\mathrm{M}+\mathrm{H})^{+}$172.1332, found 172.1331 .

\section{(1.6) Condition E}

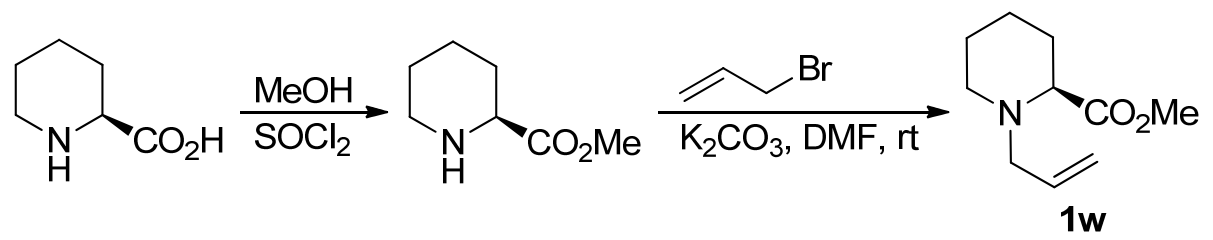

To a solution of $L$-pipecolinic acid $(1.29 \mathrm{~g}, 10.0 \mathrm{mmol})$ in methanol $(20 \mathrm{~mL})$ at $0{ }^{\circ} \mathrm{C}$ was added dropwise thionyl chloride $(3.57 \mathrm{~g}, 2.2 \mathrm{~mL}, 30.0 \mathrm{mmol})$. The mixture was heated at $70{ }^{\circ} \mathrm{C}$ for $12 \mathrm{~h}$, cooled to room temperature, and concentrated. The resulting crude (S)-methyl piperidine-2-carboxylate was dissolved in $N, N$-dimethylformamide $(20 \mathrm{~mL})$ and added allylbromide $(1.21 \mathrm{~g}, 0.86 \mathrm{~mL}, 10.0 \mathrm{mmol})$ and potassium carbonate $(4.15 \mathrm{~g}, 30.0 \mathrm{mmol})$. The mixture was stirred at room temperature for $12 \mathrm{~h}$. To the mixture was added water $(10 \mathrm{~mL})$ and ethyl acetate $(10 \mathrm{~mL})$. After separation, the aqueous layer was extracted with ethyl acetate $(3 \times 10 \mathrm{~mL})$. The organic phase was washed with brine, dried over anhydrous sodium sulfate, and concentrated. The residue was subjected to silica gel chromatography, using a mixture of ethyl acetate and petroleum ether $(1: 8)$ as the eluent, to give $(S)$-methyl 1-allylpiperidine-2-carboxylate $(\mathbf{1 w})(1.12 \mathrm{~g}, 61 \%$ yield) as a pale yellow oil. $[\alpha]_{\mathrm{D}}{ }^{20}=-50.2\left(c=0.90\right.$, EtOAc); ${ }^{1} \mathrm{H}$ NMR $\left(400 \mathrm{MHz}, \mathrm{CDCl}_{3}\right) \delta 5.94-5.83(\mathrm{~m}, 1 \mathrm{H})$, 5.19-5.11 (m, 2H), $3.72(\mathrm{~s}, 3 \mathrm{H}), 3.27-3.20(\mathrm{~m}, 1 \mathrm{H}), 3.11-3.00(\mathrm{~m}, 2 \mathrm{H}), 2.93(\mathrm{dd}, J=13.6,7.6 \mathrm{~Hz}$, $1 \mathrm{H}), 2.21-2.11(\mathrm{~m}, 1 \mathrm{H}), 1.89-1.58(\mathrm{~m}, 5 \mathrm{H}), 1.40-1.29(\mathrm{~m}, 1 \mathrm{H}) ;{ }^{13} \mathrm{C} \mathrm{NMR}\left(100 \mathrm{MHz}, \mathrm{CDCl}_{3}\right) \delta 174.2$, 134.8, 118.2, 65.0, 59.8, 51.7, 50.9, 29.8, 25.3, 22.8; HRMS (ESI) calcd for $\mathrm{C}_{10} \mathrm{H}_{18} \mathrm{NO}_{2}{ }^{+}(\mathrm{M}+\mathrm{H})^{+}$ 184.1332, found 184.1331 .

(2) Addition to Iminiums ${ }^{9}$

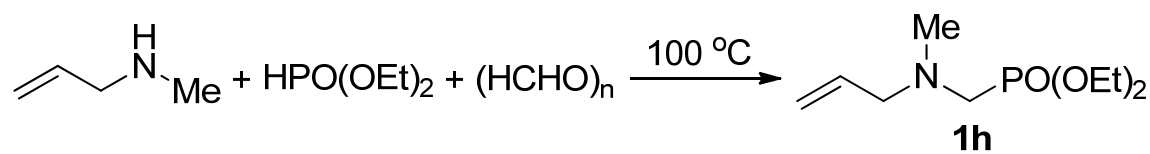

A mixture of $N$-allylmethylamine $(711 \mathrm{mg}, 0.96 \mathrm{~mL}, 10.0 \mathrm{mmol})$, diethyl phosphate $(1.38 \mathrm{~g}, 1.3$ $\mathrm{mL}, 10.0 \mathrm{mmol})$, and paraformaldehyde $(300 \mathrm{mg}, 10.0 \mathrm{mmol})$ was stirred at $100{ }^{\circ} \mathrm{C}$ for $2 \mathrm{~h}$. The mixture was cooled to room temperature and added diethyl ether $(20 \mathrm{~mL})$ and potassium carbonate (967 mg, $7.0 \mathrm{mmol}$ ). The mixture was stirred at room temperature for $2 \mathrm{~h}$, and the organic layer was separated, dried over anhydrous sodium sulfate, and concentrated. The residue was subjected to silica 
gel chromatography, using a mixture of ethyl acetate and petroleum ether (1:3) as the eluent, to give diethyl ((allyl(methyl)amino)methyl)phosphonate (1h) (664 mg, 30\% yield) as a yellow oil. ${ }^{1} \mathrm{H}$ NMR $\left(400 \mathrm{MHz}, \mathrm{CDCl}_{3}\right) \delta 5.90-5.79(\mathrm{~m}, 1 \mathrm{H}), 5.23-5.15(\mathrm{~m}, 2 \mathrm{H}), 4.20-4.10(\mathrm{~m}, 4 \mathrm{H}), 3.14$ (dd, $J=6.4,0.8$ $\mathrm{Hz}, 2 \mathrm{H}), 2.80(\mathrm{~d}, J=11.2 \mathrm{~Hz}, 2 \mathrm{H}), 2.43(\mathrm{~s}, 3 \mathrm{H}), 1.34(\mathrm{t}, J=7.2 \mathrm{~Hz}, 6 \mathrm{H}) ;{ }^{13} \mathrm{C} \mathrm{NMR}(100 \mathrm{MHz}$, $\left.\mathrm{CDCl}_{3}\right) \delta 135.1,118.4,62.5(\mathrm{~d}, J=13.8 \mathrm{~Hz}), 62.1(\mathrm{~d}, J=6.7 \mathrm{~Hz}), 52.0(\mathrm{~d}, J=162.4 \mathrm{~Hz}), 44.3,44.2$, $16.6(\mathrm{~d}, J=5.8 \mathrm{~Hz})$; HRMS (ESI) calcd for $\mathrm{C}_{9} \mathrm{H}_{21} \mathrm{NO}_{3} \mathrm{P}^{+}(\mathrm{M}+\mathrm{H})^{+} 222.1254$, found 222.1254 .

\section{Optimization of the Reaction Conditions}

For the procedure, see below. The results were summarized in the following table.

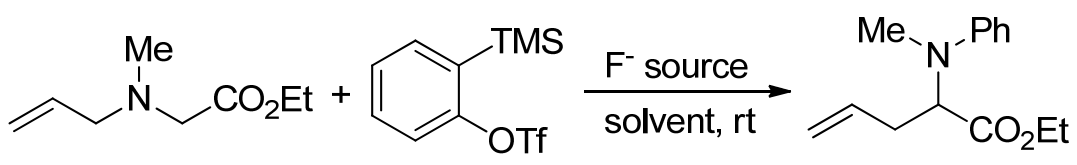

$1 \mathrm{2a}$

3a

$\begin{array}{llllllll}\text { entry } & \text { fluoride source } & \text { solvent } & \text { yield }(\%)^{b} & \text { entry } & \text { fluoride source } & \text { solvent } & \text { yield }(\%)^{b} \\ 1 & \text { CsF } & \text { MeCN } & 56 & 7 & \text { TBAF } & \text { EtOAc } & 74 \\ 2 & \text { KF } & \text { MeCN } & \text { trace } & 8 & \text { TBAF } & \text { PhMe } & 72 \\ 3 & \text { KF/18-crown-6 } & \text { MeCN } & 43 & 9 & \text { TBAF } & \text { DCE } & 75 \\ 4 & \text { TBAF } & \text { MeCN } & 76 & 10 & \text { TBAF } & \text { DMF } & 60 \\ 5 & \text { TBAF } & \text { THF } & 85 & 11 & \text { TBAF } & \text { DMSO } & 37 \\ 6 & \text { TBAF } & \text { DME } & 77 & & & & \end{array}$

${ }^{a}$ Reaction conditions: $1 \mathrm{a}(0.20 \mathrm{mmol}), 2 \mathrm{a}(0.28 \mathrm{mmol}), \mathrm{F}^{-}$source $(0.30 \mathrm{mmol})$, solvent $(1.0 \mathrm{~mL}), \mathrm{rt}$, 3 h. ${ }^{b}$ Isolated yields.

General Procedure for the Aryne-Mediated [2,3]-Sigmatropic Rearrangement of Tertiary Allylic Amines

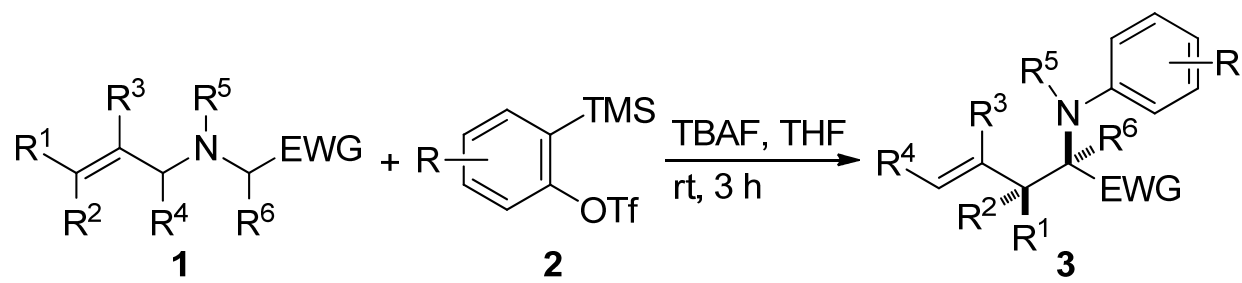

A mixture of amine $1(0.20 \mathrm{mmol})$, TBAF $(78.4 \mathrm{mg}, 0.30 \mathrm{mmol}, 1.0 \mathrm{M}$ in THF, $0.30 \mathrm{~mL})$ in tetrahydrofuran $(1.0 \mathrm{~mL})$ was added 2-(trimethylsilyl)aryl triflate $2(0.28 \mathrm{mmol})$. The mixture was stirred at room temperature for $3 \mathrm{~h}$. The mixture was concentrated and purified by silica gel chromatography, using a mixture of ethyl acetate and petroleum ether (1:2 to 1:60) as the eluent, to give homoallylic amine 3 .

The absolute configuration of compounds 3aa and 3ac was assigned by single crystal X-ray analysis of their derivatives (see below). The absolute configuration of compound 3ab was assigned by analogy. The relative stereochemistry of compounds $\mathbf{3 m}$ and $\mathbf{3 n}$ was determined by ${ }^{1} \mathrm{H}$ NMR spectroscopic analysis (see below). 


\section{Analytical Data for the Products}<smiles>C=CCC(C(=O)OCC)N(C)c1ccccc1</smiles>

3a

Ethyl 2-(methyl(phenyl)amino)pent-4-enoate (3a) ${ }^{10}$ was obtained $(39.6 \mathrm{mg}, 85 \%$ yield) as a yellow oil. ${ }^{1} \mathrm{H}$ NMR $\left(400 \mathrm{MHz}, \mathrm{CDCl}_{3}\right) \delta$ 7.26-7.20 (m, 2H), 6.83-6.72 (m, 3H), 5.83-5.72 (m, 1H), 5.18-5.03 (m, 2H), $4.43(\mathrm{dd}, J=8.8,6.4 \mathrm{~Hz}, 1 \mathrm{H}), 4.19-4.11(\mathrm{~m}, 2 \mathrm{H}), 2.90(\mathrm{~s}, 3 \mathrm{H}), 2.77-2.68(\mathrm{~m}, 1 \mathrm{H})$, 2.65-2.56 (m, 1H), $1.22(\mathrm{t}, J=7.2 \mathrm{~Hz}, 3 \mathrm{H}) ;{ }^{13} \mathrm{C} \mathrm{NMR}\left(100 \mathrm{MHz}, \mathrm{CDCl}_{3}\right) \delta 172.3,150.2,134.4$, 129.2, 117.7, 113.6, 61.8, 60.9, 34.3, 33.0, 14.4; HRMS (ESI) calcd for $\mathrm{C}_{14} \mathrm{H}_{20} \mathrm{NO}_{2}{ }^{+}(\mathrm{M}+\mathrm{H})^{+}$ 234.1489 , found 234.1488 .

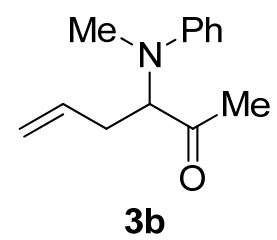

3-(Methyl(phenyl)amino)hex-5-en-2-one (3b) was obtained $(25.2 \mathrm{mg}, 62 \%$ yield) as a yellow oil. ${ }^{1} \mathrm{H}$ NMR $\left(400 \mathrm{MHz}, \mathrm{CDCl}_{3}\right) \delta$ 7.29-7.24 (m, 2H), 6.82-6.76 (m, 3H), 5.79-5.68 (m, $\left.1 \mathrm{H}\right)$, 5.12-4.97 (m, 2H), $4.33(\mathrm{dd}, J=8.0,6.0 \mathrm{~Hz}, 1 \mathrm{H}), 2.79(\mathrm{~s}, 3 \mathrm{H}), 2.72-2.64(\mathrm{~m}, 1 \mathrm{H}), 2.48-2.39(\mathrm{~m}, 1 \mathrm{H})$, $2.11(\mathrm{~s}, 3 \mathrm{H}) ;{ }^{13} \mathrm{C}$ NMR $\left(100 \mathrm{MHz}, \mathrm{CDCl}_{3}\right) \delta 208.2,149.3,134.9,129.5,117.7,117.5,113.0,68.1$, 33.5, 31.8, 27.9; HRMS (ESI) calcd for $\mathrm{C}_{13} \mathrm{H}_{18} \mathrm{NO}^{+}(\mathrm{M}+\mathrm{H})^{+}$204.1383, found 204.1382.<smiles>C=CCC(C(=O)c1ccccc1)N(C)c1ccccc1</smiles>

2-(Methyl(phenyl)amino)-1-phenylpent-4-en-1-one (3c) was obtained (39.3 $\mathrm{mg}, 74 \%$ yield) as a yellow oil. ${ }^{1} \mathrm{H}$ NMR $\left(400 \mathrm{MHz}, \mathrm{CDCl}_{3}\right) \delta$ 7.87-7.82 (m, 2H), 7.52-7.47 (m, 1H), 7.38-7.24 (m, 4H), 6.88-6.78 (m, 3H), 5.92-5.80 (m, 1H), 5.23-5.13 (m, 2H), 5.07-5.02 (m, 1H), 2.89-2.80 (m, 1H), 2.71 $(\mathrm{s}, 3 \mathrm{H}), 2.59-2.49(\mathrm{~m}, 1 \mathrm{H}) ;{ }^{13} \mathrm{C} \mathrm{NMR}\left(100 \mathrm{MHz}, \mathrm{CDCl}_{3}\right) \delta 198.2,148.7,136.2,135.1,133.4,129.7$, 128.7, 128.4, 117.9, 117.7, 113.1, 62.9, 32.9, 32.0; HRMS (ESI) calcd for $\mathrm{C}_{18} \mathrm{H}_{20} \mathrm{NO}^{+}(\mathrm{M}+\mathrm{H})^{+}$ 266.1539 , found 266.1539 .<smiles>C=CCC(C(N)=O)N(C)c1ccccc1</smiles> 
2-(Methyl(phenyl)amino)pent-4-enamide (3d) was obtained $(28.2 \mathrm{mg}, 69 \%$ yield) as a pale yellow oil. ${ }^{1} \mathrm{H}$ NMR $\left(400 \mathrm{MHz}, \mathrm{CDCl}_{3}\right) \delta$ 7.29-7.23 (m, 2H), 6.85-6.78 (m, 3H), 6.32 (s, br, 1H), 5.85 (s, br, 1H), 5.76-5.65 (m, 1H), 5.09-4.94 (m, 2H), 4.35 (dd, $J=10.0,5.2 \mathrm{~Hz}, 1 \mathrm{H}), 2.96-2.89$ (m, $1 \mathrm{H}), 2.88(\mathrm{~s}, 3 \mathrm{H}), 2.61-2.51(\mathrm{~m}, 1 \mathrm{H}) ;{ }^{13} \mathrm{C} \mathrm{NMR}\left(100 \mathrm{MHz}, \mathrm{CDCl}_{3}\right) \delta 175.0,149.6,135.3,129.5$, 118.5, 117.3, 113.9, 64.0, 34.0, 32.7; HRMS (ESI) calcd for $\mathrm{C}_{12} \mathrm{H}_{17} \mathrm{~N}_{2} \mathrm{O}^{+}(\mathrm{M}+\mathrm{H})^{+}$205.1335, found 205.1334 .<smiles>C=CCC(C(=O)NC)N(C)c1ccccc1</smiles>

$\mathrm{N}$-Methyl-2-(methyl(phenyl)amino)pent-4-enamide (3e) was obtained (23.1 $\mathrm{mg}, 53 \%$ yield) as a yellow solid. m.p. 90-92 ${ }^{\circ} \mathrm{C}$; ${ }^{1} \mathrm{H}$ NMR $\left(400 \mathrm{MHz}, \mathrm{CDCl}_{3}\right) \delta$ 7.29-7.22 (m, 2H), 6.84-6.74 (m, 3H), 6.49 (s, br, 1H), 5.75-5.64 (m, 1H), 5.07-4.91 (m, 2H), 4.30 (dd, $J=10.0,4.8 \mathrm{~Hz}, 1 \mathrm{H}), 3.03-2.95$ (m, $1 \mathrm{H}), 2.85(\mathrm{~s}, 3 \mathrm{H}), 2.82(\mathrm{~d}, J=4.8 \mathrm{~Hz}, 3 \mathrm{H}), 2.60-2.50(\mathrm{~m}, 1 \mathrm{H}) ;{ }^{13} \mathrm{C} \mathrm{NMR}\left(100 \mathrm{MHz}, \mathrm{CDCl}_{3}\right) \delta 172.6$, 149.7, 135.5, 129.5, 118.4, 117.2, 113.9, 64.4, 34.0, 32.9, 26.3; HRMS (ESI) calcd for $\mathrm{C}_{13} \mathrm{H}_{19} \mathrm{~N}_{2} \mathrm{O}^{+}$ $(\mathrm{M}+\mathrm{H})^{+}$219.1492, found 219.1493 .<smiles>C=CCC(C(=O)N1CCOCC1)N(C)c1ccccc1</smiles>

2-(Methyl(phenyl)amino)-1-morpholinopent-4-en-1-one (3f) was obtained (28.0 mg, 51\% yield) as a yellow oil. ${ }^{1} \mathrm{H} \mathrm{NMR}\left(400 \mathrm{MHz}, \mathrm{CDCl}_{3}\right) \delta 7.31-7.23(\mathrm{~m}, 2 \mathrm{H}), 6.81-6.74(\mathrm{~m}, 3 \mathrm{H}), 5.90-5.78(\mathrm{~m}$, $1 \mathrm{H}), 5.18-5.02(\mathrm{~m}, 2 \mathrm{H}), 4.46(\mathrm{dd}, J=8.4,5.6 \mathrm{~Hz}, 1 \mathrm{H}), 3.87-3.79(\mathrm{~m}, 1 \mathrm{H}), 3.76-3.69(\mathrm{~m}, 1 \mathrm{H})$, 3.62-3.44 (m, 3H), 3.39-3.18 (m, 3H), $2.83(\mathrm{~s}, 3 \mathrm{H}), 2.81-2.75(\mathrm{~m}, 1 \mathrm{H}), 2.44-2.36(\mathrm{~m}, 1 \mathrm{H}) ;{ }^{13} \mathrm{C} \mathrm{NMR}$ $\left(100 \mathrm{MHz}, \mathrm{CDCl}_{3}\right) \delta 169.0,148.3,135.4,129.7,117.8,117.5,112.6,67.2,66.9,59.1,46.1,42.7$, 32.8, 32.5; HRMS (ESI) calcd for $\mathrm{C}_{16} \mathrm{H}_{23} \mathrm{~N}_{2} \mathrm{O}_{2}{ }^{+}(\mathrm{M}+\mathrm{H})^{+}$275.1754, found 275.1755.

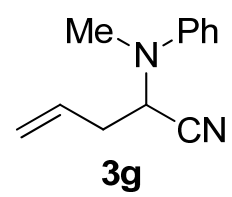

2-(Methyl(phenyl)amino)pent-4-enenitrile $(3 \mathbf{g})^{11}$ was obtained $(29.8 \mathrm{mg}, 88 \%$ yield) as a yellow oil. ${ }^{1} \mathrm{H}$ NMR (400 MHz, $\left.\mathrm{CDCl}_{3}\right) \delta$ 7.33-7.27 (m, 2H), 6.97-6.91 (m, 3H), 5.88-5.77 (m, 1H), 5.31-5.21 (m, 2H), $4.52(\mathrm{t}, J=7.6 \mathrm{~Hz}, 1 \mathrm{H}), 2.92(\mathrm{~s}, 3 \mathrm{H}), 2.69-2.63(\mathrm{~m}, 2 \mathrm{H}) ;{ }^{13} \mathrm{C} \mathrm{NMR}(100 \mathrm{MHz}$, $\left.\mathrm{CDCl}_{3}\right) \delta 149.2,131.8,129.5,121.1,119.9,117.5,117.0,54.4,36.2$, 34.6; HRMS (ESI) calcd for $\mathrm{C}_{12} \mathrm{H}_{28} \mathrm{~N}^{+}(\mathrm{M}+\mathrm{H})^{+}$186.2216, found 186.2218. 
<smiles>C=CCC(POCC)N(C)c1ccccc1</smiles>

$3 \mathrm{~h}$

Diethyl (1-(methyl(phenyl)amino)but-3-en-1-yl)phosphonate (3h) was obtained $(35.7 \mathrm{mg}, 60 \%$ yield) as a colorless oil. ${ }^{1} \mathrm{H}$ NMR $\left(400 \mathrm{MHz}, \mathrm{CDCl}_{3}\right) \delta 7.25-7.19(\mathrm{~m}, 2 \mathrm{H}), 6.81(\mathrm{~d}, J=8.4 \mathrm{~Hz}, 2 \mathrm{H})$, $6.74(\mathrm{t}, J=7.2 \mathrm{~Hz}, 1 \mathrm{H}), 5.74-5.62(\mathrm{~m}, 1 \mathrm{H}), 5.14-4.96(\mathrm{~m}, 2 \mathrm{H}), 4.22(\mathrm{ddd}, J=15.6,11.2,4.4 \mathrm{~Hz}, 1 \mathrm{H})$, 4.12-3.94 (m, 4H), 2.97 (d, $J=1.2 \mathrm{~Hz}, 3 \mathrm{H}), 2.82-2.69(\mathrm{~m}, 1 \mathrm{H}), 2.65-2.56(\mathrm{~m}, 1 \mathrm{H}), 1.27$ (t, $J=7.2 \mathrm{~Hz}$, $3 \mathrm{H}), 1.19(\mathrm{t}, J=7.2 \mathrm{~Hz}, 3 \mathrm{H}) ;{ }^{13} \mathrm{C} \mathrm{NMR}\left(100 \mathrm{MHz}, \mathrm{CDCl}_{3}\right) \delta 150.5(\mathrm{~d}, J=3.6 \mathrm{~Hz}), 134.6(\mathrm{~d}, J=16.3$ $\mathrm{Hz}), 129.2,117.7(\mathrm{~d}, J=1.4 \mathrm{~Hz}), 117.6,113.5(\mathrm{~d}, J=1.2 \mathrm{~Hz}), 62.7(\mathrm{~d}, J=7.1 \mathrm{~Hz}), 61.8(\mathrm{~d}, J=7.3$ $\mathrm{Hz}), 56.7(\mathrm{~d}, J=154.0 \mathrm{~Hz}), 32.8,32.1(\mathrm{~d}, J=7.0 \mathrm{~Hz}), 16.7(\mathrm{~d}, J=5.5 \mathrm{~Hz}), 16.6(\mathrm{~d}, J=5.7 \mathrm{~Hz})$; HRMS (ESI) calcd for $\mathrm{C}_{15} \mathrm{H}_{25} \mathrm{NO}_{3} \mathrm{P}^{+}(\mathrm{M}+\mathrm{H})^{+} 298.1567$, found 298.1568.

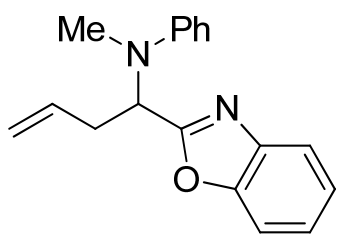

3i

$N$-(1-(Benzo[d]oxazol-2-yl)but-3-en-1-yl)- $N$-methylaniline (3i) was obtained $(43.4 \mathrm{mg}, 78 \%$ yield) as a yellow oil. ${ }^{1} \mathrm{H}$ NMR $\left(400 \mathrm{MHz}, \mathrm{CDCl}_{3}\right) \delta 7.77-7.69(\mathrm{~m}, 1 \mathrm{H}), 7.51-7.44(\mathrm{~m}, 1 \mathrm{H})$, 7.36-7.22 (m, 4H), $6.95(\mathrm{~d}, J=8.0 \mathrm{~Hz}, 2 \mathrm{H}), 6.80(\mathrm{t}, J=7.2 \mathrm{~Hz}, 1 \mathrm{H}), 5.93-5.80(\mathrm{~m}, 1 \mathrm{H}), 5.26-5.16(\mathrm{~m}$, 2H), $5.08(\mathrm{~d}, J=10.0 \mathrm{~Hz}, 1 \mathrm{H}), 3.08-2.87(\mathrm{~m}, 5 \mathrm{H}) ;{ }^{13} \mathrm{C} \mathrm{NMR}\left(100 \mathrm{MHz}, \mathrm{CDCl}_{3}\right) \delta 165.5,150.8$, 149.9, 140.9, 134.0, 129.4, 125.2, 124.4, 120.2, 118.2, 114.0, 110.9, 57.6, 35.0, 32.6; HRMS (ESI) calcd for $\mathrm{C}_{18} \mathrm{H}_{19} \mathrm{~N}_{2} \mathrm{O}^{+}(\mathrm{M}+\mathrm{H})^{+} 279.1492$, found 279.1492 .<smiles>C=CCC(c1ccc([N+](=O)[O-])cc1)N(C)c1ccccc1</smiles>

3j

$N$-Methyl- $N$-(1-(4-nitrophenyl)but-3-en-1-yl)aniline (3j) was obtained (24.3 $\mathrm{mg}, 43 \%$ yield) as a yellow oil. ${ }^{1} \mathrm{H}$ NMR $\left(400 \mathrm{MHz}, \mathrm{CDCl}_{3}\right) \delta 8.15(\mathrm{~d}, J=8.8 \mathrm{~Hz}, 2 \mathrm{H}), 7.42(\mathrm{~d}, J=8.8 \mathrm{~Hz}, 2 \mathrm{H})$, 7.28-7.21 (m, 2H), 6.85-6.73 (m, 3H), 5.87-5.75 (m, 1H), 5.21-5.04 (m, 3H), 2.88-2.71 (m, 5H); ${ }^{13} \mathrm{C}$ NMR $\left(100 \mathrm{MHz}, \mathrm{CDCl}_{3}\right) \delta 150.1,149.1,147.1,134.9,129.4,128.1,123.8,117.7,113.5,61.5,36.3$, 32.3; HRMS (ESI) calcd for $\mathrm{C}_{17} \mathrm{H}_{19} \mathrm{~N}_{2} \mathrm{O}_{2}^{+}(\mathrm{M}+\mathrm{H})^{+}$283.1441, found 283.1447.

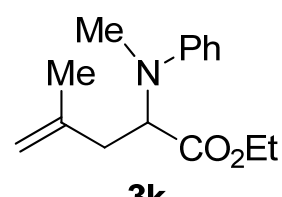

3k

Ethyl 4-methyl-2-(methyl(phenyl)amino)pent-4-enoate (3k) was obtained (31.2 $\mathrm{mg}, 63 \%$ yield) as a yellow oil. ${ }^{1} \mathrm{H}$ NMR $\left(400 \mathrm{MHz}, \mathrm{CDCl}_{3}\right) \delta 7.27-7.20(\mathrm{~m}, 2 \mathrm{H}), 6.82(\mathrm{~d}, J=8.4 \mathrm{~Hz}, 2 \mathrm{H}), 6.75(\mathrm{t}, J$ 
$=7.2 \mathrm{~Hz}, 1 \mathrm{H}), 4.81-4.77(\mathrm{~m}, 2 \mathrm{H}), 4.57(\mathrm{dd}, J=8.8,6.4 \mathrm{~Hz}, 1 \mathrm{H}), 4.19-4.10(\mathrm{~m}, 2 \mathrm{H}), 2.91(\mathrm{~s}, 3 \mathrm{H})$, $2.69(\mathrm{dd}, J=14.8,6.4 \mathrm{~Hz}, 1 \mathrm{H}), 2.57(\mathrm{dd}, J=14.8,8.8 \mathrm{~Hz}, 1 \mathrm{H}), 1.73(\mathrm{~s}, 3 \mathrm{H}), 1.22(\mathrm{t}, J=7.2 \mathrm{~Hz}, 3 \mathrm{H})$; ${ }^{13} \mathrm{C} \mathrm{NMR}\left(100 \mathrm{MHz}, \mathrm{CDCl}_{3}\right) \delta 172.6,150.0,141.7,129.3,117.7,113.6,113.3,60.9,60.1,38.1,32.9$, 22.3, 14.4; HRMS (ESI) calcd for $\mathrm{C}_{15} \mathrm{H}_{22} \mathrm{NO}_{2}^{+}(\mathrm{M}+\mathrm{H})^{+} 248.1645$, found 248.1647.

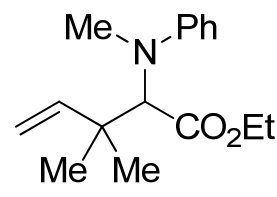

3I

Ethyl 3,3-dimethyl-2-(methyl(phenyl)amino)pent-4-enoate (3I) was obtained $(29.8 \mathrm{mg}, 57 \%$ yield) as a yellow oil. ${ }^{1} \mathrm{H} \mathrm{NMR}\left(400 \mathrm{MHz}, \mathrm{CDCl}_{3}\right) \delta 7.28-7.20(\mathrm{~m}, 2 \mathrm{H}), 6.84(\mathrm{~d}, J=8.4 \mathrm{~Hz}, 2 \mathrm{H})$, $6.74(\mathrm{t}, J=7.2 \mathrm{~Hz}, 1 \mathrm{H}), 6.26-6.15(\mathrm{~m}, 1 \mathrm{H}), 5.13-5.01(\mathrm{~m}, 2 \mathrm{H}), 4.34(\mathrm{~s}, 1 \mathrm{H}), 4.18-4.10(\mathrm{~m}, 2 \mathrm{H}), 2.97$ $(\mathrm{s}, 3 \mathrm{H}), 1.28-1.18(\mathrm{~m}, 9 \mathrm{H}) ;{ }^{13} \mathrm{C}$ NMR $\left(100 \mathrm{MHz}, \mathrm{CDCl}_{3}\right) \delta 170.7,151.1,145.2,129.2,117.4,113.3$, 112.7, 69.3, 60.4, 41.9, 35.6, 27.2, 24.6, 14.5; HRMS (ESI) calcd for $\mathrm{C}_{16} \mathrm{H}_{24} \mathrm{NO}_{2}^{+}(\mathrm{M}+\mathrm{H})^{+}$ 262.1802 , found 262.1801 .<smiles>C=CC(c1ccccc1)C(C(=O)OCC)N(C)c1ccccc1</smiles>

$3 m$

Ethyl 2-(methyl(phenyl)amino)-3-phenylpent-4-enoate (3m) was obtained (40.8 $\mathrm{mg}, 66 \%$ yield) as a 94:6 mixture of syn:anti stereomers. The syn stereoisomer was identified according to the coupling constant of the hydrogen with $\delta$ 4.68. ${ }^{12}$ Yellow oil; ${ }^{1} \mathrm{H}$ NMR $\left(400 \mathrm{MHz}, \mathrm{CDCl}_{3}\right) \delta$ 7.34-7.19 (m, 7H), $6.93(\mathrm{~d}, J=8.4 \mathrm{~Hz}, 2 \mathrm{H}), 6.81-6.76(\mathrm{~m}, 1 \mathrm{H}), 6.04-5.93(\mathrm{~m}, 1 \mathrm{H}), 5.11-5.02(\mathrm{~m}, 2 \mathrm{H})$, $4.68(\mathrm{~d}, J=11.2 \mathrm{~Hz}, 1 \mathrm{H}), 4.07(\mathrm{dd}, J=11.2,8.0 \mathrm{~Hz}, 1 \mathrm{H}), 3.92-3.83(\mathrm{~m}, 2 \mathrm{H}), 3.03(\mathrm{~s}, 3 \mathrm{H}), 0.92(\mathrm{t}, J$ $=7.2 \mathrm{~Hz}, 3 \mathrm{H})$; Partial ${ }^{1} \mathrm{H}$ NMR for the anti stereoisomer, $\delta 4.21-4.13(\mathrm{~m}, 2 \mathrm{H}), 2.77(\mathrm{~s}, 3 \mathrm{H}), 1.24(\mathrm{t}, J$ $=7.2 \mathrm{~Hz}, 3 \mathrm{H})$; HRMS $(\mathrm{ESI})$ calcd for $\mathrm{C}_{20} \mathrm{H}_{24} \mathrm{NO}_{2}^{+}(\mathrm{M}+\mathrm{H})^{+} 310.1802$, found 310.1801 .<smiles>CCOC(=O)C(C1C=CCCC1)N(C)c1ccccc1</smiles>

$3 n$

Ethyl 2-(cyclohex-2-en-1-yl)-2-(methyl(phenyl)amino)acetate (3n) was obtained (32.3 mg, 59\% yield) as 56:44 mixture of syn:anti stereomers. The syn stereoisomer was identified according to the coupling constant of the hydrogen with $\delta 4.06$. Yellow oil; ${ }^{1} \mathrm{H}$ NMR $\left(400 \mathrm{MHz}, \mathrm{CDCl}_{3}\right) \delta 7.26-7.20$ $(\mathrm{m}, 2 \mathrm{H}), 6.90-6.83(\mathrm{~m}, 2 \mathrm{H}), 6.74(\mathrm{t}, J=7.2 \mathrm{~Hz}, 1 \mathrm{H}), 5.76-5.70(\mathrm{~m}, 1 \mathrm{H}), 5.58-5.48(\mathrm{~m}, 1 \mathrm{H}), 4.19-4.09$ $(\mathrm{m}, 2 \mathrm{H}), 4.06(\mathrm{~d}, J=11.2 \mathrm{~Hz}, 1 \mathrm{H}), 2.97(\mathrm{~s}, 3 \mathrm{H}), 2.89-2.80(\mathrm{~m}, 1 \mathrm{H}), 2.05-1.96(\mathrm{~m}, 2 \mathrm{H}), 1.85-1.75(\mathrm{~m}$, $1 \mathrm{H}), 1.73-1.45(\mathrm{~m}, 2 \mathrm{H}), 1.38-1.19(\mathrm{~m}, 4 \mathrm{H})$; Partial ${ }^{1} \mathrm{H}$ NMR for the anti stereoisomer, $\delta$ 5.85-5.79 (m, $1 \mathrm{H}), 2.91(\mathrm{~s}, 3 \mathrm{H})$; HRMS (ESI) calcd for $\mathrm{C}_{17} \mathrm{H}_{24} \mathrm{NO}_{2}{ }^{+}(\mathrm{M}+\mathrm{H})^{+} 274.1802$, found 274.1800. 
<smiles>C=CCC(C(=O)OCC)N(CCC)c1ccccc1</smiles>

Ethyl 2-(phenyl(propyl)amino)pent-4-enoate (3o) was obtained (37.1 $\mathrm{mg}, 71 \%$ yield) as a colorless oil. ${ }^{1} \mathrm{H}$ NMR $\left(400 \mathrm{MHz}, \mathrm{CDCl}_{3}\right) \delta$ 7.26-7.18 (m, 2H), 6.81-6.72 (m, 3H), 5.87-5.75 (m, 1H), 5.17-5.03 (m, 2H), $4.31(\mathrm{dd}, J=8.0,7.2 \mathrm{~Hz}, 1 \mathrm{H}), 4.14(\mathrm{q}, J=7.2 \mathrm{~Hz}, 2 \mathrm{H}), 3.31-3.15(\mathrm{~m}, 2 \mathrm{H})$, 2.78-2.69 (m, 1H), 2.65-2.55 (m, 1H), 1.67-1.51 (m, 2H), $1.21(\mathrm{t}, J=7.2 \mathrm{~Hz}, 3 \mathrm{H}), 0.90(\mathrm{t}, J=7.2 \mathrm{~Hz}$, $3 \mathrm{H}) ;{ }^{13} \mathrm{C} \mathrm{NMR}\left(100 \mathrm{MHz}, \mathrm{CDCl}_{3}\right) \delta 172.6,148.6,134.7,129.2,117.9,117.6,114.8,63.0,60.9,49.2$, 34.5, 21.4, 14.4, 11.5; HRMS (ESI) calcd for $\mathrm{C}_{16} \mathrm{H}_{24} \mathrm{NO}_{2}^{+}(\mathrm{M}+\mathrm{H})^{+}$262.1802, found 262.1802.

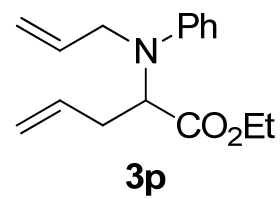

Ethyl 2-(allyl(phenyl)amino)pent-4-enoate (3p) was obtained (40.5 $\mathrm{mg}, 78 \%$ yield) as a pale yellow oil. ${ }^{1} \mathrm{H}$ NMR (400 MHz, $\left.\mathrm{CDCl}_{3}\right) \delta$ 7.25-7.17 (m, 2H), 6.84-6.72 (m, 3H), 5.93-5.75 (m, 2H), 5.27-5.04 (m, 4H), $4.40(\mathrm{t}, J=7.2 \mathrm{~Hz}, 1 \mathrm{H}), 4.14(\mathrm{q}, J=7.2 \mathrm{~Hz}, 2 \mathrm{H}), 4.08-3.92(\mathrm{~m}, 2 \mathrm{H}), 2.78-2.69(\mathrm{~m}$, $1 \mathrm{H}), 2.66-2.56(\mathrm{~m}, 1 \mathrm{H}), 1.22(\mathrm{t}, J=7.2 \mathrm{~Hz}, 3 \mathrm{H}) ;{ }^{13} \mathrm{C} \mathrm{NMR}\left(100 \mathrm{MHz}, \mathrm{CDCl}_{3}\right) \delta 172.5,148.8,136.0$, 134.5, 129.1, 118.0, 117.8, 116.1, 114.4, 61.8, 61.0, 50.4, 34.6, 14.3; HRMS (ESI) calcd for $\mathrm{C}_{16} \mathrm{H}_{22} \mathrm{NO}_{2}^{+}(\mathrm{M}+\mathrm{H})^{+} 260.1645$, found 260.1643 .

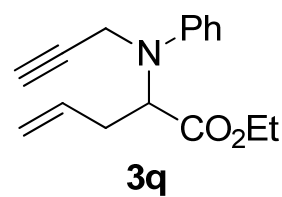

Ethyl 2-(phenyl(prop-2-yn-1-yl)amino)pent-4-enoate (3q) was obtained (25.7 $\mathrm{mg}, 50 \%$ yield) as a yellow oil. ${ }^{1} \mathrm{H}$ NMR $\left(400 \mathrm{MHz}, \mathrm{CDCl}_{3}\right) \delta 7.29-7.22(\mathrm{~m}, 2 \mathrm{H}), 6.94(\mathrm{~d}, J=8.0 \mathrm{~Hz}, 2 \mathrm{H}), 6.82(\mathrm{t}, J=$ $7.2 \mathrm{~Hz}, 1 \mathrm{H}), 5.92-5.80(\mathrm{~m}, 1 \mathrm{H}), 5.20-5.05(\mathrm{~m}, 2 \mathrm{H}), 4.39(\mathrm{t}, J=7.6 \mathrm{~Hz}, 1 \mathrm{H}), 4.27-4.02(\mathrm{~m}, 4 \mathrm{H})$, 2.81-2.67 (m, 2H), $2.23(\mathrm{t}, J=2.4 \mathrm{~Hz}, 1 \mathrm{H}), 1.23(\mathrm{t}, J=7.2 \mathrm{~Hz}, 3 \mathrm{H}) ;{ }^{13} \mathrm{C} \mathrm{NMR}\left(100 \mathrm{MHz}, \mathrm{CDCl}_{3}\right) \delta$ 172.2, 148.3, 134.1, 129.3, 119.0, 118.0, 114.6, 81.3, 71.9, 61.6, 61.1, 37.3, 34.9, 14.3; HRMS (ESI) calcd for $\mathrm{C}_{16} \mathrm{H}_{20} \mathrm{NO}_{2}^{+}(\mathrm{M}+\mathrm{H})^{+} 258.1489$, found 258.1488 .<smiles>C=CCC(C(=O)OCC)N(CC(=O)OCC)c1ccccc1</smiles>

Ethyl 2-((2-ethoxy-2-oxoethyl)(phenyl)amino)pent-4-enoate (3r) was obtained (25.6 mg, 42\% yield) as a yellow oil. ${ }^{1} \mathrm{H}$ NMR $\left(400 \mathrm{MHz}, \mathrm{CDCl}_{3}\right) \delta 7.25-7.20(\mathrm{~m}, 2 \mathrm{H}), 6.82-6.73(\mathrm{~m}, 3 \mathrm{H})$, 5.90-5.79 (m, 1H), 5.18-5.06 (m, 2H), $4.45(\mathrm{t}, J=7.2 \mathrm{~Hz}, 1 \mathrm{H}), 4.27-4.05(\mathrm{~m}, 6 \mathrm{H}), 2.70-2.61(\mathrm{~m}, 2 \mathrm{H})$, 1.29-1.22 (m, 6H); ${ }^{13} \mathrm{C}$ NMR (100 MHz, $\left.\mathrm{CDCl}_{3}\right) \delta 172.2,171.6,148.7,133.8,129.4,118.8,118.1$, 
113.9, 61.1, 61.0, 49.6, 35.1, 14.4, 14.3; HRMS (ESI) calcd for $\mathrm{C}_{17} \mathrm{H}_{24} \mathrm{NO}_{4}^{+}(\mathrm{M}+\mathrm{H})^{+} 306.1700$, found 306.1701 .

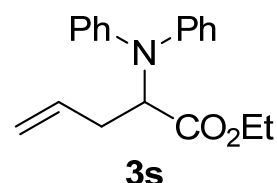

Ethyl 2-(diphenylamino)pent-4-enoate (3s) was obtained (37.2 $\mathrm{mg}, 63 \%$ yield) as a pale yellow oil. ${ }^{1} \mathrm{H}$ NMR (400 MHz, $\left.\mathrm{CDCl}_{3}\right) \delta$ 7.30-7.22 (m, 4H), 7.03-6.94 (m, 6H), 5.86-5.73 (m, 1H), 5.09-5.00 (m, 2H), $4.62(\mathrm{t}, J=7.2 \mathrm{~Hz}, 1 \mathrm{H}), 4.25-4.11(\mathrm{~m}, 2 \mathrm{H}), 2.63(\mathrm{t}, J=7.2 \mathrm{~Hz}, 2 \mathrm{H}), 1.22(\mathrm{t}, J=$ $7.2 \mathrm{~Hz}, 3 \mathrm{H}) ;{ }^{13} \mathrm{C}$ NMR $\left(100 \mathrm{MHz}, \mathrm{CDCl}_{3}\right) \delta 172.8,146.7,134.6,129.2,122.8,122.4,117.8,62.8$, 61.2, 34.5, 14.3; HRMS (ESI) calcd for $\mathrm{C}_{19} \mathrm{H}_{22} \mathrm{NO}_{2}{ }^{+}(\mathrm{M}+\mathrm{H})^{+}$296.1645, found 296.1646.<smiles>C=CCC1C(=O)NCCN1Pc1ccccc1</smiles>

3-Allyl-4-phenylpiperazin-2-one (3t) was obtained $\left(26.4 \mathrm{mg}, 61 \%\right.$ yield) as a yellow oil. ${ }^{1} \mathrm{H}$ NMR (400 MHz, $\left.\mathrm{CDCl}_{3}\right) \delta$ 7.38-7.14 (m, 3H), 6.89-6.81 (m, 3H), 5.96-5.83 (m, 1H), 5.14-5.02 (m, $2 \mathrm{H}), 4.31(\mathrm{t}, J=6.0 \mathrm{~Hz}, 1 \mathrm{H}), 3.64-3.48(\mathrm{~m}, 3 \mathrm{H}), 3.43-3.32(\mathrm{~m}, 1 \mathrm{H}), 2.72-2.64(\mathrm{~m}, 2 \mathrm{H}) ;{ }^{13} \mathrm{C} \mathrm{NMR}$ $\left(100 \mathrm{MHz} \mathrm{CDCl}_{3}\right) \delta 172.0,148.4,134.5,129.5,119.4,117.9,115.5,61.3,41.9,40.4,35.8$; HRMS (ESI) calcd for $\mathrm{C}_{13} \mathrm{H}_{16} \mathrm{~N}_{2} \mathrm{O}^{+}(\mathrm{M}+\mathrm{H})^{+} 217.1335$, found 217.1335 .
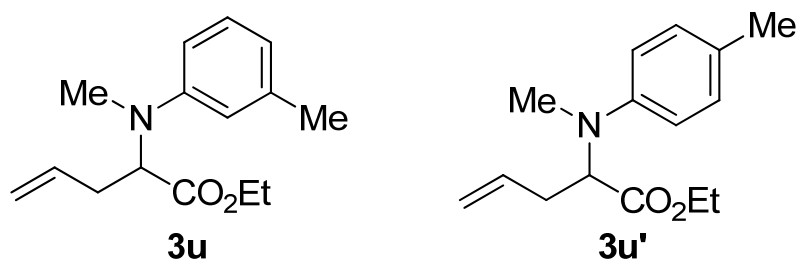

A 56:44 mixture of ethyl 2-(methyl(m-tolyl)amino)pent-4-enoate (3u) and ethyl 2-(methyl( $p$ tolyl)amino)pent-4-enoate (3u') was obtained (37.5 mg, 76\% yield) as a yellow oil. ${ }^{1} \mathrm{H}$ NMR (400 $\left.\mathrm{MHz}, \mathrm{CDCl}_{3}\right) \delta$ 7.15-7.09 (m, 1H), 6.64-6.56 (m, 3H), 5.84-5.71 (m, 1H), 5.18-5.02 (m, 2H), 4.45-4.34 (m, 1H), 4.21-4.08 (m, 2H), $2.89(\mathrm{~s}, 3 \mathrm{H}), 2.76-2.54(\mathrm{~m}, 2 \mathrm{H}), 2.31(\mathrm{~s}, 3 \mathrm{H}), 1.26-1.19(\mathrm{~m}$, $3 \mathrm{H})$; Partial ${ }^{1} \mathrm{H}$ NMR for amine 3u', $\delta 7.04(\mathrm{~d}, J=8.8 \mathrm{~Hz}, 2 \mathrm{H}), 6.74(\mathrm{~d}, J=8.8 \mathrm{~Hz}, 2 \mathrm{H}), 2.88(\mathrm{~s}, 3 \mathrm{H})$, 2.25 (s, 3H); HRMS (ESI) calcd for $\mathrm{C}_{15} \mathrm{H}_{22} \mathrm{NO}_{2}{ }^{+}(\mathrm{M}+\mathrm{H})^{+} 248.1645$, found 248.1644.<smiles>C=CCC(C(=O)OCC)N(C)c1ccc(C)c(C)c1</smiles> 
Ethyl 2-((3,4-dimethylphenyl)(methyl)amino)pent-4-enoate (3v) was obtained $(41.8 \mathrm{mg}, 80 \%$ yield) as a yellow oil. ${ }^{1} \mathrm{H}$ NMR $\left(400 \mathrm{MHz}, \mathrm{CDCl}_{3}\right) \delta 6.99(\mathrm{~d}, J=8.4 \mathrm{~Hz}, 1 \mathrm{H}), 6.66-6.56(\mathrm{~m}, 2 \mathrm{H})$, 5.83-5.72 (m, 1H), 5.18-5.02 (m, 2H), $4.37(\mathrm{dd}, J=8.8,6.8 \mathrm{~Hz}, 1 \mathrm{H}), 4.21-4.08(\mathrm{~m}, 2 \mathrm{H}), 2.88(\mathrm{~s}, 3 \mathrm{H})$, 2.74-2.65 (m, 1H), 2.63-2.54 (m, 1H), $2.22(\mathrm{~s}, 3 \mathrm{H}), 2.16(\mathrm{~s}, 3 \mathrm{H}), 1.22(\mathrm{t}, J=7.2 \mathrm{~Hz}, 3 \mathrm{H}) ;{ }^{13} \mathrm{C}$ NMR $\left(100 \mathrm{MHz} \mathrm{CDCl}_{3}\right) \delta 172.4,148.4,137.2,134.5,130.3,126.0,117.6,115.7,111.5,62.2,60.8,34.2$, 33.2, 20.5, 18.7, 14.4; HRMS (ESI) calcd for $\mathrm{C}_{16} \mathrm{H}_{24} \mathrm{NO}_{2}^{+}(\mathrm{M}+\mathrm{H})^{+}$262.1802, found 262.1803.

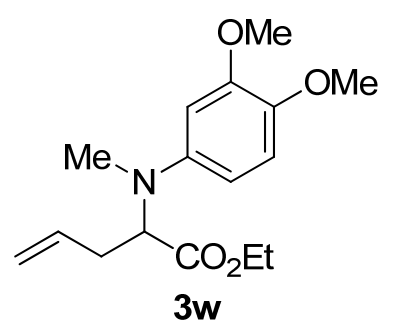

Ethyl 2-((3,4-dimethoxyphenyl)(methyl)amino)pent-4-enoate (3w) was obtained $(44.0 \mathrm{mg}, 75 \%$ yield) as a yellow oil. ${ }^{1} \mathrm{H}$ NMR $\left(400 \mathrm{MHz}, \mathrm{CDCl}_{3}\right) \delta 6.78(\mathrm{~d}, J=8.8 \mathrm{~Hz}, 1 \mathrm{H}), 6.48(\mathrm{~s}, 1 \mathrm{H}), 6.36$ (dd, $J=8.8,2.4 \mathrm{~Hz}, 1 \mathrm{H}), 5.86-5.74(\mathrm{~m}, 1 \mathrm{H}), 5.19-5.05(\mathrm{~m}, 2 \mathrm{H}), 4.28(\mathrm{dd}, J=8.8,6.8 \mathrm{~Hz}, 1 \mathrm{H}), 4.20-4.10$ $(\mathrm{m}, 2 \mathrm{H}), 3.87(\mathrm{~s}, 3 \mathrm{H}), 3.82(\mathrm{~s}, 3 \mathrm{H}), 2.88(\mathrm{~s}, 3 \mathrm{H}), 2.74-2.65(\mathrm{~m}, 1 \mathrm{H}), 2.64-2.54(\mathrm{~m}, 1 \mathrm{H}), 1.23(\mathrm{t}, J=$ $7.2 \mathrm{~Hz}, 3 \mathrm{H}) ;{ }^{13} \mathrm{C} \mathrm{NMR}\left(100 \mathrm{MHz}, \mathrm{CDCl}_{3}\right) \delta 172.3,149.7,145.4,142.2,134.5,117.7,112.7,106.3$, 100.6, 63.3, 60.8, 56.6, 55.9, 34.2, 33.8, 14.4; HRMS (ESI) calcd for $\mathrm{C}_{16} \mathrm{H}_{24} \mathrm{NO}_{4}{ }^{+}(\mathrm{M}+\mathrm{H})^{+}$ 294.1700 , found 294,1698 .<smiles>C=CCC(C(=O)OCC)N(C)c1ccc(F)c(F)c1</smiles>

Ethyl 2-((3,4-difluorophenyl)(methyl)amino)pent-4-enoate (3x) was obtained $(21.5 \mathrm{mg}, 40 \%$ yield) as a yellow oil. ${ }^{1} \mathrm{H} \mathrm{NMR}\left(400 \mathrm{MHz}, \mathrm{CDCl}_{3}\right) \delta 7.00(\mathrm{dd}, J=19.2,9.2 \mathrm{~Hz}, 1 \mathrm{H}), 6.59$ (ddd, $J=$ 9.6, 6.4, $2.8 \mathrm{~Hz}, 1 \mathrm{H}), 6.48-6.43(\mathrm{~m}, 1 \mathrm{H}), 5.80-5.69(\mathrm{~m}, 1 \mathrm{H}), 5.19-5.05(\mathrm{~m}, 2 \mathrm{H}), 4.28$ (dd, $J=9.2,6.4$ $\mathrm{Hz}, 1 \mathrm{H}), 4.21-4.12(\mathrm{~m}, 2 \mathrm{H}), 2.86(\mathrm{~s}, 3 \mathrm{H}), 2.76-2.67(\mathrm{~m}, 1 \mathrm{H}), 2.64-2.55(\mathrm{~m}, 1 \mathrm{H}), 1.23(\mathrm{t}, J=7.2 \mathrm{~Hz}$, $3 \mathrm{H}) ;{ }^{13} \mathrm{C} \mathrm{NMR}\left(100 \mathrm{MHz}, \mathrm{CDCl}_{3}\right) \delta 171.8,150.7$ (dd, $\left.J=242.8,13.0 \mathrm{~Hz}\right), 147.4(\mathrm{dd}, J=8.2,1.9$ $\mathrm{Hz}), 143.2(\mathrm{dd}, J=236.3,12.8 \mathrm{~Hz}), 134.0,118.0,117.3$ (dd, $J=17.6,1.9 \mathrm{~Hz}), 108.9$ (dd, $J=5.4,3.0$ $\mathrm{Hz}), 102.9(\mathrm{~d}, J=21.1 \mathrm{~Hz}), 62.3,61.1,34.2,33.4,14.4$; HRMS (ESI) calcd for $\mathrm{C}_{14} \mathrm{H}_{18} \mathrm{NO}_{2} \mathrm{~F}_{2}^{+}(\mathrm{M}+$ H) ${ }^{+} 270.1300$, found 270.1299 .<smiles>C=CCC(C(=O)OCC)N(C)c1ccc2ccccc2c1</smiles>

3y 
Ethyl 2-(methyl(naphthalen-2-yl)amino)pent-4-enoate (3y) was obtained ( $42.5 \mathrm{mg}, 75 \%$ yield) as a yellow oil. ${ }^{1} \mathrm{H}$ NMR $\left(400 \mathrm{MHz}, \mathrm{CDCl}_{3}\right) \delta 7.72-7.63(\mathrm{~m}, 3 \mathrm{H}), 7.39-7.33(\mathrm{~m}, 1 \mathrm{H}), 7.25-7.17(\mathrm{~m}$, $2 \mathrm{H}), 7.02(\mathrm{~d}, J=2.4 \mathrm{~Hz}, 1 \mathrm{H}), 5.86-5.74(\mathrm{~m}, 1 \mathrm{H}), 5.20-5.03(\mathrm{~m}, 2 \mathrm{H}), 4.57$ (dd, $J=8.8,6.4 \mathrm{~Hz}, 1 \mathrm{H})$, 4.21-4.11 (m, 2H), $3.00(\mathrm{~s}, 3 \mathrm{H}), 2.81-2.73(\mathrm{~m}, 1 \mathrm{H}), 2.70-2.61(\mathrm{~m}, 1 \mathrm{H}), 1.21(\mathrm{t}, J=7.2 \mathrm{~Hz}, 3 \mathrm{H}) ;{ }^{13} \mathrm{C}$ NMR $\left(100 \mathrm{MHz}, \mathrm{CDCl}_{3}\right) \delta 172.2,148.0,135.0,134.3,128.9,127.5,126.5,126.4,122.7,117.8$, 117.0, 108.1, 62.2, 61.0, 34.2, 33.3, 14.4; HRMS (ESI) calcd for $\mathrm{C}_{18} \mathrm{H}_{22} \mathrm{NO}_{2}{ }^{+}(\mathrm{M}+\mathrm{H})^{+} 284.1645$, found 284.1643 .

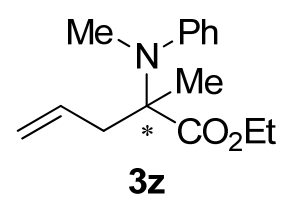

Ethyl 2-methyl-2-(methyl(phenyl)amino)pent-4-enoate (3z) was obtained (31.1 $\mathrm{mg}, 63 \%$ yield) as a yellow oil. $[\alpha]_{\mathrm{D}}{ }^{20}=-3.5\left(c=0.40\right.$, EtOAc); ${ }^{1} \mathrm{H}$ NMR $\left(400 \mathrm{MHz}, \mathrm{CDCl}_{3}\right) \delta 7.27-7.18(\mathrm{~m}, 2 \mathrm{H})$, 7.06-6.95 (m, 3H), 5.83-5.71 (m, 1H), 5.12-5.03 (m, 2H), 4.23-4.12 (m, 2H), $2.92(\mathrm{~s}, 3 \mathrm{H}), 2.66(\mathrm{dd}, J$ $=14.0,6.8 \mathrm{~Hz}, 1 \mathrm{H}), 2.56(\mathrm{dd}, J=14.0,7.6 \mathrm{~Hz}, 1 \mathrm{H}), 1.35(\mathrm{~s}, 3 \mathrm{H}), 1.22(\mathrm{t}, J=7.2 \mathrm{~Hz}, 3 \mathrm{H}) ;{ }^{13} \mathrm{C} \mathrm{NMR}$ $\left(100 \mathrm{MHz}, \mathrm{CDCl}_{3}\right) \delta 175.2,150.0,133.9,128.4,124.3,122.7,118.5,65.4,60.8,42.0,37.7,21.7$, 14.3; HRMS (ESI) calcd for $\mathrm{C}_{15} \mathrm{H}_{22} \mathrm{NO}_{2}{ }^{+}(\mathrm{M}+\mathrm{H})^{+}$248.1645, found 248.1644. The ee was determined to be $14 \%$ by HPLC analysis (Chiralpak OJ column, $\lambda=254 \mathrm{~nm}$, hexane/isopropanol $=$ $87 / 13$, flow rate $=0.7 \mathrm{~mL} / \mathrm{min}): \mathrm{t}_{\mathrm{R}}($ major $)=7.5 \mathrm{~min}, \mathrm{t}_{\mathrm{R}}($ minor $)=8.5 \mathrm{~min}$.

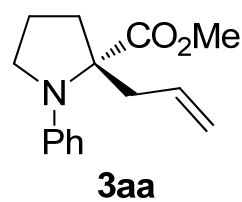

(S)-Methyl 2-allyl-1-phenylpyrrolidine-2-carboxylate (3aa) was obtained (29.4 $\mathrm{mg}, 60 \%$ yield) as a yellow oil. $[\alpha]_{\mathrm{D}}{ }^{20}=+6.3\left(c=0.70\right.$, EtOAc); ${ }^{1} \mathrm{H}$ NMR $\left(400 \mathrm{MHz}, \mathrm{CDCl}_{3}\right) \delta 7.21-7.16(\mathrm{~m}, 2 \mathrm{H})$, 6.71-6.66 (m, 1H), 6.56-6.51 (m, 2H), 5.62-5.50 (m, 1H), 5.07-5.00 (m, 2H), 3.68 (s, 3H), 3.53-3.45 $(\mathrm{m}, 2 \mathrm{H}), 3.14-3.06(\mathrm{~m}, 1 \mathrm{H}), 2.73-2.66(\mathrm{~m}, 1 \mathrm{H}), 2.31-2.23(\mathrm{~m}, 1 \mathrm{H}), 2.31-1.93(\mathrm{~m}, 3 \mathrm{H}) ;{ }^{13} \mathrm{C} \mathrm{NMR}$ $\left(100 \mathrm{MHz}, \mathrm{CDCl}_{3}\right) \delta 177.1,145.7,133.5,129.1,118.9,116.7,113.3,67.9,52.5,51.0,37.9,37.7$, 23.0; HRMS (ESI) calcd for $\mathrm{C}_{15} \mathrm{H}_{20} \mathrm{NO}_{2}{ }^{+}(\mathrm{M}+\mathrm{H})^{+}$246.1489, found 246.1487. The ee was determined to be $90 \%$ by HPLC analysis (Chiralpak OD column, $\lambda=254 \mathrm{~nm}$, hexane/isopropanol $=$ $95 / 5$, flow rate $=0.8 \mathrm{~mL} / \mathrm{min}): \mathrm{t}_{\mathrm{R}}($ minor $)=6.0 \mathrm{~min}, \mathrm{t}_{\mathrm{R}}($ major $)=6.5 \mathrm{~min}$.

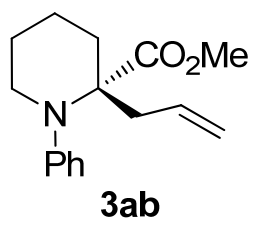

(S)-Methyl 2-allyl-1-phenylpiperidine-2-carboxylate (3ab) was obtained (33.2 $\mathrm{mg}, 64 \%$ yield) as a yellow oil; $[\alpha]_{\mathrm{D}}{ }^{20}=+60.1\left(c=1.9\right.$, EtOAc); ${ }^{1} \mathrm{H}$ NMR $\left(400 \mathrm{MHz}, \mathrm{CDCl}_{3}\right) \delta 7.26-7.19(\mathrm{~m}, 2 \mathrm{H})$, $7.11(\mathrm{~d}, J=7.6 \mathrm{~Hz}, 2 \mathrm{H}), 7.02(\mathrm{t}, J=7.2 \mathrm{~Hz}, 1 \mathrm{H}), 5.64-5.52(\mathrm{~m}, 1 \mathrm{H}), 4.99-4.89(\mathrm{~m}, 2 \mathrm{H}), 3.69$ (s, 3H), 3.49-3.38 (m, 1H), 3.25-3.15 (m, 1H), $2.62(\mathrm{dd}, J=14.0,6.8 \mathrm{~Hz}, 1 \mathrm{H}), 2.45(\mathrm{dd}, J=14.0,7.2 \mathrm{~Hz}$, 
$1 \mathrm{H}), 2.08-2.00(\mathrm{~m}, 1 \mathrm{H}), 1.75-1.54(\mathrm{~m}, 5 \mathrm{H}) ;{ }^{13} \mathrm{C} \mathrm{NMR}\left(100 \mathrm{MHz}, \mathrm{CDCl}_{3}\right) \delta 175.5,150.4,133.8$, 128.3, 126.6, 123.6, 118.1, 65.7, 51.5, 51.3, 41.5, 34.5, 26.4, 21.4; HRMS (ESI) calcd for $\mathrm{C}_{16} \mathrm{H}_{22} \mathrm{NO}_{2}^{+}(\mathrm{M}+\mathrm{H})^{+} 260.1645$, found 260.1644 . The ee was determined to be $90 \%$ by HPLC analysis (Chiralpak OJ column, $\lambda=254 \mathrm{~nm}$, hexane/isopropanol $=90 / 10$, flow rate $=1.0 \mathrm{~mL} / \mathrm{min}$ ): $\mathrm{t}_{\mathrm{R}}($ major $)=6.9 \mathrm{~min}, \mathrm{t}_{\mathrm{R}}($ minor $)=9.6 \mathrm{~min}$.

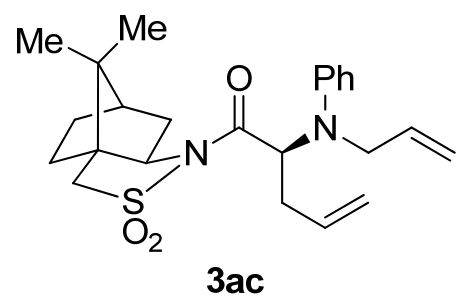

(2S)-2-(allyl(phenyl)amino)-1-((6R,7a R)-8,8-dimethyl-2,2-dioxidohexahydro-1H-3a,6-methano benzo[c]isothiazol-1-yl)pent-4-en-1-one (3ac) was obtained $(56.5 \mathrm{mg}, 66 \%$ yield) as an $84: 16$ mixture of diastereomers. Yellow oil; $[\alpha]_{\mathrm{D}}{ }^{20}=-31.2\left(c=0.80\right.$, EtOAc); ${ }^{1} \mathrm{H} \mathrm{NMR}\left(400 \mathrm{MHz}, \mathrm{CDCl}_{3}\right)$ $\delta$ 7.23-7.15 (m, 2H), 6.94-6.89 (m, 2H), 6.82-6.71 (m, 1H), 5.93-5.73 (m, 2H), 5.26-5.02 (m, 5H), 4.22-4.01 (m, 2H), 3.91-3.83 (m, 1H), 3.53-3.36 (m, 2H), 2.89-2.77 (m, 1H), 2.66-2.49 (m, 1H), 2.07-2.01 (m, 2H), 1.97-1.76 (m, 3H), 1.44-1.21 (m, 2H), $1.15(\mathrm{~s}, 3 \mathrm{H}), 0.96(\mathrm{~s}, 3 \mathrm{H})$; Partial ${ }^{1} \mathrm{H}$ NMR for the minor diastereoisomer, $\delta 0.91(\mathrm{~s}, 3 \mathrm{H})$; HRMS (ESI) calcd for $\mathrm{C}_{24} \mathrm{H}_{33} \mathrm{~N}_{2} \mathrm{O}_{3} \mathrm{~S}^{+}(\mathrm{M}+\mathrm{H})^{+}$ 429.2206, found 429.2206.

\section{Chemical Transformations of the Products}

(1) Preparation of Amine 5

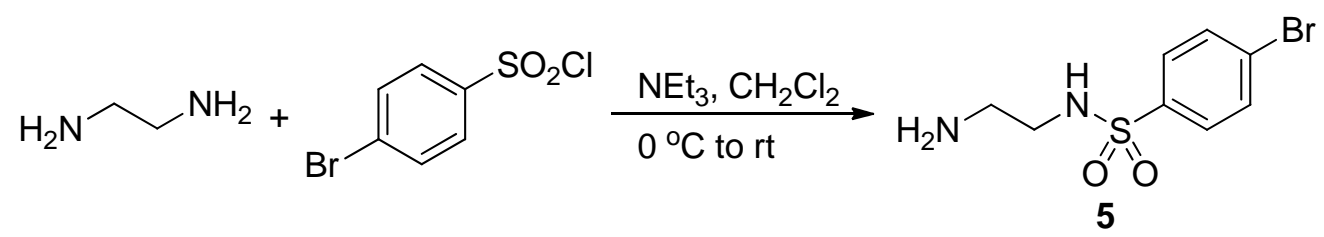

To a solution of ethylenediamine $(601 \mathrm{mg}, 0.67 \mathrm{~mL}, 10.0 \mathrm{mmol})$ in dichloromethane $(20 \mathrm{~mL})$ was added triethylamine $(3.04 \mathrm{~g}, 4.17 \mathrm{~mL}, 30.0 \mathrm{mmol})$. The mixture was cooled to $0{ }^{\circ} \mathrm{C}$ and added dropwise a solution of the 4-bromobenzenesulfonyl chloride $(1.28 \mathrm{~g}, 5.00 \mathrm{mmol})$ in dichloromethane $(2.0 \mathrm{~mL})$. The mixture was stirred at room temperature for $14 \mathrm{~h}$, washed with aqueous $10 \%$ sodium carbonate $(20 \mathrm{~mL})$, water $(3 \times 10 \mathrm{~mL})$, dried over anhydrous sodium sulfate, and concentrated. The residue was subjected to silica gel chromatography, using a mixture of methanol and dichloromethane (1:20) as the eluent, to give $N$-(2-aminoethyl)-4-bromobenzenesulfonamide (5) (2.50 g, 89\% yield) as white solid. m.p. $135-137{ }^{\circ} \mathrm{C} ;{ }^{1} \mathrm{H}$ NMR $\left(400 \mathrm{MHz}, \mathrm{CDCl}_{3}\right) \delta 7.78-7.58$ (m, $4 \mathrm{H}), 3.30-3.02(\mathrm{~m}, 3 \mathrm{H}), 3.01-2.92(\mathrm{~m}, 2 \mathrm{H}), 2.85-2.75(\mathrm{~m}, 2 \mathrm{H}) ;{ }^{13} \mathrm{C} \mathrm{NMR}\left(100 \mathrm{MHz}, \mathrm{CDCl}_{3}\right) \delta 139.1$, 132.5, 128.6, 127.6, 45.3, 41.0; HRMS (ESI) calcd for $\mathrm{C}_{8} \mathrm{H}_{12} \mathrm{O}_{2} \mathrm{~N}_{2} \mathrm{SBr}^{+}(\mathrm{M}+\mathrm{H})^{+}$278.9797, found 278.9805.

(2) Synthesis of Amide 6 


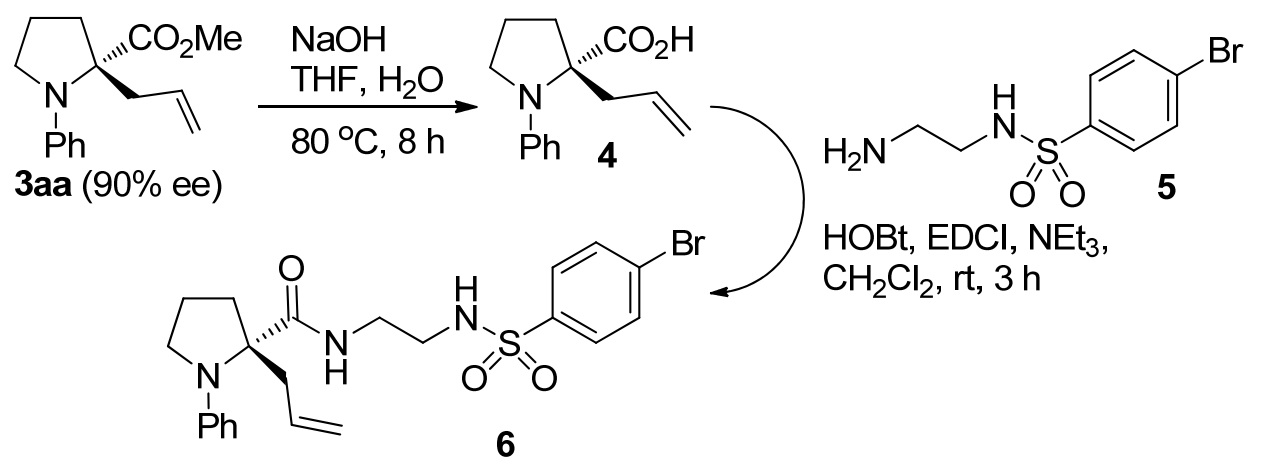

To a solution of ester 3aa $(245 \mathrm{mg}, 1.0 \mathrm{mmol})$ in tetrahydrofuran $(2.0 \mathrm{~mL})$ and methanol $(3.0$ $\mathrm{mL})$ were added sodium hydroxide $(200 \mathrm{mg}, 5.0 \mathrm{mmol})$ and water $(2.5 \mathrm{~mL})$. The mixture was heated at $80{ }^{\circ} \mathrm{C}$ for $8 \mathrm{~h}$, cooled to room temperature, added hydrochloric acid $(1.0 \mathrm{M})$ until $\mathrm{pH}=7.0$, and extracted with ethyl acetate $(3 \times 5 \mathrm{~mL})$. The organic layer was dried over anhydrous sodium sulfate and concentrated to give crude acid 4 . Crude acid 4 was transferred into a $10 \mathrm{~mL}$ round-bottom flask, to which were added amine 5 (332 mg, $1.2 \mathrm{mmol})$, HOBt (203 mg, $1.5 \mathrm{mmol})$, EDCI (288 mg, 1.5 $\mathrm{mmol})$, and triethylamine $(152 \mathrm{mg}, 0.21 \mathrm{~mL}, 1.5 \mathrm{mmol})$. The mixture was stirred at room temperature for $3 \mathrm{~h}$, concentrated, and subjected to silica gel chromatography, using a mixture of ethyl acetate and petroleum ether (1:2) as the eluent, to give (S)-2-allyl- $N$-(2-(4-bromophenylsulfonamido)ethyl)-1-phenylpyrrolidine-2-carboxamide (6) (383 mg, 78\% yield (2 steps)) as a white solid, which was subjected to recrystallization and subsequent single crystal X-ray analysis (see below). m.p. $137-139{ }^{\circ} \mathrm{C} ;[\alpha]_{\mathrm{D}}{ }^{20}=+18.3\left(c=0.70\right.$, EtOAc); ${ }^{1} \mathrm{H} \mathrm{NMR}\left(400 \mathrm{MHz}, \mathrm{CDCl}_{3}\right) \delta 7.63-7.60$ (m, 4H), 7.24-7.18 (m, 2H), $7.07(\mathrm{~s}, \mathrm{br}, 1 \mathrm{H}), 6.81(\mathrm{t}, J=7.2 \mathrm{~Hz}, 1 \mathrm{H}), 6.61(\mathrm{~d}, J=8.0 \mathrm{~Hz}, 2 \mathrm{H}), 5.50$ (s, br, 1H), 5.39-5.27 (m, 1H), 5.01-4.91 (m, 2H), $3.54(\mathrm{t}, J=8.0 \mathrm{~Hz}, 1 \mathrm{H}), 3.39-3.27(\mathrm{~m}, 3 \mathrm{H})$, 3.11-2.95 (m, 3H), $2.71(\mathrm{dd}, J=14.8,8.8 \mathrm{~Hz}, 1 \mathrm{H}), 2.38-2.27(\mathrm{~m}, 1 \mathrm{H}), 2.18-2.09(\mathrm{~m}, 1 \mathrm{H}), 2.03-1.85$ $(\mathrm{m}, 2 \mathrm{H}) ;{ }^{13} \mathrm{C} \mathrm{NMR}\left(100 \mathrm{MHz}, \mathrm{CDCl}_{3}\right) \delta 177.3,144.9,139.1,132.8,132.5,129.3,128.6,127.7$, $119.8,118.3,114.8,69.1,50.8,43.6,39.4,37.9,35.8,23.0$; HRMS (ESI) calcd for $\mathrm{C}_{22} \mathrm{H}_{27} \mathrm{~N}_{3} \mathrm{O}_{3} \mathrm{SBr}^{+}$ $(\mathrm{M}+\mathrm{H})^{+}$492.0951, found 492.0949 .

(3) Synthesis of Cyclic Allylic Amine 7 


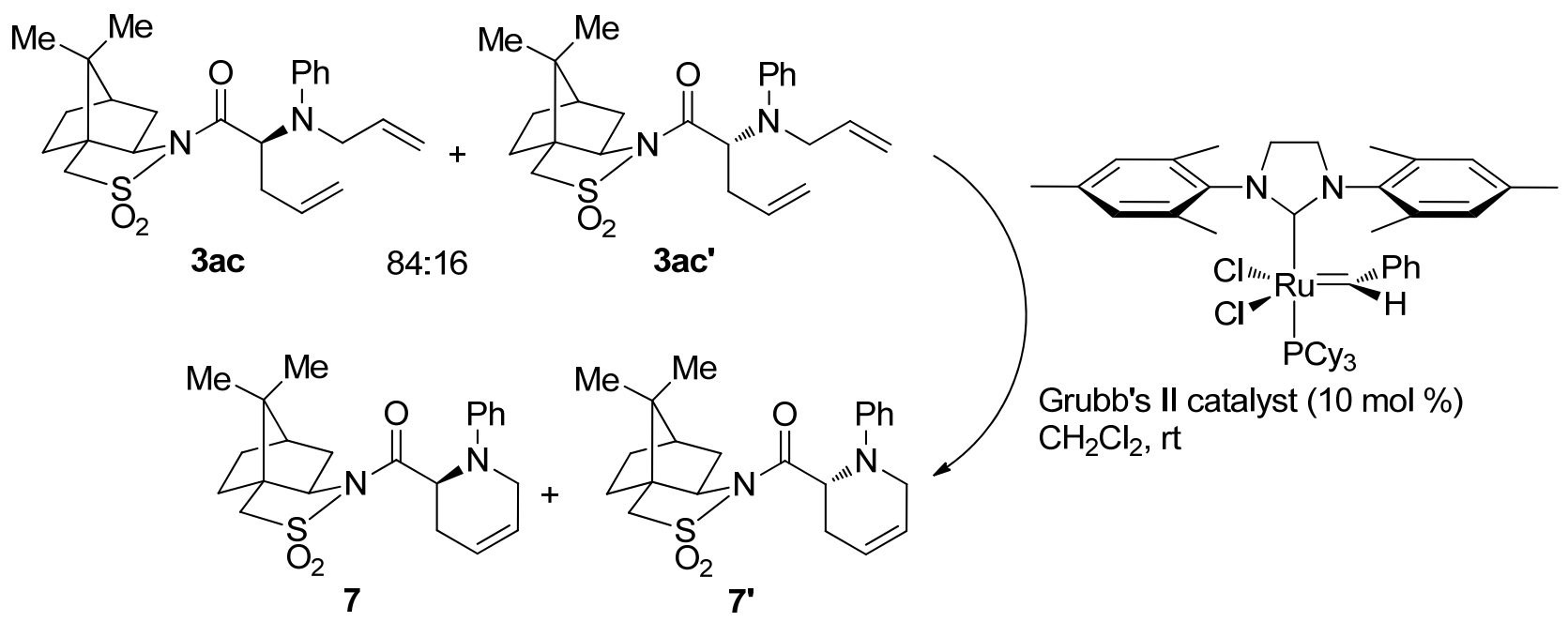

A mixture of diene 3ac (141 mg, $0.33 \mathrm{mmol})$ and Grubb's II catalyst (25.5 mg, $10 \mathrm{~mol} \%$ ) in dichloromethane $(2.0 \mathrm{~mL})$ was stirred under nitrogen at room temperature for $14 \mathrm{~h}$. The mixture was concentrated and purified by silica gel chromatography, using a mixture of ethyl acetate and petroleum ether (1:15) as the eluent, to give cyclic allylic amines 7 (99.2 mg, 75\% yield) and 7' (19.5 $\mathrm{mg}, 15 \%$ yield). Cyclic allylic amine 7 was subjected to single crystal X-ray analysis (see below).

$((6 R, 7 \mathrm{a} R)-8,8$-Dimethyl-2,2-dioxidohexahydro-1H-3a,6-methanobenzo[c]isothiazol-1-yl $)((S)-1$ phenyl-1,2,3,6-tetrahydropyridin-2-yl)methanone (7): white solid; m.p. 88-90 ${ }^{\circ} \mathrm{C} ;[\alpha]_{\mathrm{D}}{ }^{20}=-53.0(c=$ 0.60, EtOAc); ${ }^{1} \mathrm{H}$ NMR (400 MHz, $\left.\mathrm{CDCl}_{3}\right) \delta 7.29-7.20(\mathrm{~m}, 2 \mathrm{H}), 6.96(\mathrm{~d}, J=8.8 \mathrm{~Hz}, 2 \mathrm{H}), 6.78(\mathrm{t}, J=$ $7.2 \mathrm{~Hz}, 1 \mathrm{H}), 5.96-5.89(\mathrm{~m}, 1 \mathrm{H}), 5.79-5.70(\mathrm{~m}, 1 \mathrm{H}), 5.25(\mathrm{dd}, J=7.2,1.6 \mathrm{~Hz}, 1 \mathrm{H}), 4.12-3.97(\mathrm{~m}, 2 \mathrm{H})$, $3.84(\mathrm{dd}, J=6.8,5.6 \mathrm{~Hz}, 1 \mathrm{H}), 3.50(\mathrm{~d}, J=13.6 \mathrm{~Hz}, 1 \mathrm{H}), 3.45(\mathrm{~d}, J=13.6 \mathrm{~Hz}, 1 \mathrm{H}), 2.89-2.68(\mathrm{~m}$, $2 \mathrm{H}), 2.02-1.77(\mathrm{~m}, 5 \mathrm{H}), 1.43-1.23(\mathrm{~m}, 2 \mathrm{H}), 1.14(\mathrm{~s}, 3 \mathrm{H}), 0.95(\mathrm{~s}, 3 \mathrm{H}) ;{ }^{13} \mathrm{C} \mathrm{NMR}\left(100 \mathrm{MHz}, \mathrm{CDCl}_{3}\right) \delta$ $172.9,148.9,129.3,126.0,119.7,118.5,113.5,65.1,54.5,53.0,48.7,47.9,46.4,44.5,38.4,32.6$, 28.9, 26.6, 20.7, 19.9; HRMS (ESI) calcd for $\mathrm{C}_{22} \mathrm{H}_{29} \mathrm{~N}_{2} \mathrm{O}_{3} \mathrm{~S}^{+}(\mathrm{M}+\mathrm{H})^{+}$401.1893, found 401.1892.

$((6 R, 7 \mathrm{a} R)-8,8$-dimethyl-2,2-dioxidohexahydro-1 $H$-3a,6-methanobenzo[ $c]$ isothiazol-1-yl $)((R)-1$ phenyl-1,2,3,6-tetrahydropyridin-2-yl)methanone (7'): yellow oil; $[\alpha]_{\mathrm{D}}{ }^{20}=-40.4(c=0.50$, EtOAc); ${ }^{1} \mathrm{H}$ NMR (400 MHz, $\left.\mathrm{CDCl}_{3}\right) \delta 7.25-7.17(\mathrm{~m}, 2 \mathrm{H}), 7.03(\mathrm{~d}, J=8.8 \mathrm{~Hz}, 2 \mathrm{H}), 6.80(\mathrm{t}, J=7.2 \mathrm{~Hz}, 1 \mathrm{H})$, 5.99-5.92 (m, 1H), 5.82-5.75 (m, 1H), $5.28(\mathrm{t}, J=4.4 \mathrm{~Hz}, 1 \mathrm{H}), 4.02-3.90(\mathrm{~m}, 2 \mathrm{H}), 3.82(\mathrm{dd}, J=7.6$, $5.2 \mathrm{~Hz}, 1 \mathrm{H}), 3.53(\mathrm{~d}, J=13.6 \mathrm{~Hz}, 1 \mathrm{H}), 3.44(\mathrm{~d}, J=13.6 \mathrm{~Hz}, 1 \mathrm{H}), 2.79-2.68(\mathrm{~m}, 2 \mathrm{H}), 1.95-1.68(\mathrm{~m}$, $5 \mathrm{H}), 1.42-1.21(\mathrm{~m}, 2 \mathrm{H}), 1.04(\mathrm{~s}, 3 \mathrm{H}), 0.93(\mathrm{~s}, 3 \mathrm{H}) ;{ }^{13} \mathrm{C} \mathrm{NMR}\left(100 \mathrm{MHz}, \mathrm{CDCl}_{3}\right) \delta 172.3,148.9$, 129.3, 124.8, 120.0, 119.2, 114.7, 65.7, 53.1, 53.0, 48.8, 47.9, 46.4, 44.4, 38.5, 32.9, 26.5, 26.4, 20.5, 20.0; HRMS (ESI) calcd for $\mathrm{C}_{22} \mathrm{H}_{29} \mathrm{~N}_{2} \mathrm{O}_{3} \mathrm{~S}^{+}(\mathrm{M}+\mathrm{H})^{+} 401.1893$, found 401.1890 .

(4) Synthesis of Cyclopropane 9

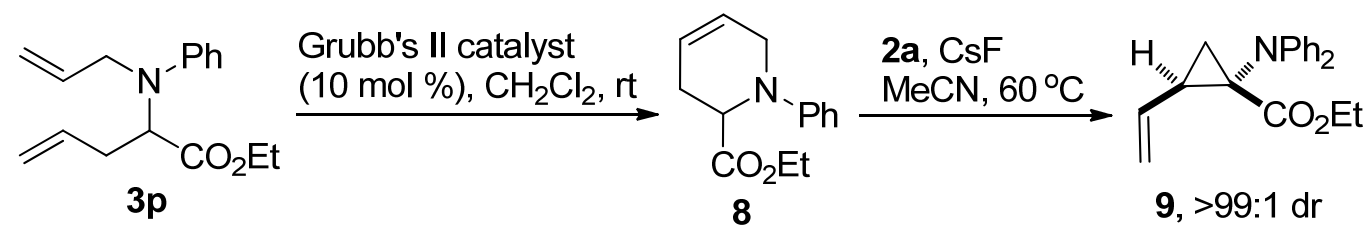


A mixture of diene 3p (130 mg, $0.50 \mathrm{mmol})$ and Grubb's II catalyst $(42.4 \mathrm{mg}, 10 \mathrm{~mol} \%)$ in dichloromethane $(3.0 \mathrm{~mL})$ was stirred under nitrogen at room temperature for $14 \mathrm{~h}$. The mixture was concentrated and purified by silica gel chromatography, using a mixture of ethyl acetate and petroleum ether (1:20) as the eluent, to give ethyl 1-phenyl-1,2,3,6-tetrahydropyridine-2- carboxylate (8) $\left(102 \mathrm{mg}, 88 \%\right.$ yield) as a yellow solid. m.p. $71-73{ }^{\circ} \mathrm{C} ;{ }^{1} \mathrm{H}$ NMR $\left(400 \mathrm{MHz}, \mathrm{CDCl}_{3}\right) \delta 7.31-7.22$ $(\mathrm{m}, 2 \mathrm{H}), 6.91-6.77(\mathrm{~m}, 3 \mathrm{H})$, 5.91-5.78 (m, 2H), 4.74-4.67 (m, 1H), 4.16-4.02 (m, 2H), 3.97-3.86 (m, 2H), 2.77-2.61 (m, 2H), $1.17(\mathrm{t}, J=7.2 \mathrm{~Hz}, 3 \mathrm{H}) ;{ }^{13} \mathrm{C} \mathrm{NMR}\left(100 \mathrm{MHz}, \mathrm{CDCl}_{3}\right) \delta 172.7,149.6,129.3$, 125.2, 121.9, 118.7, 113.9, 60.9, 54.6, 45.4, 28.3, 14.3; HRMS (ESI) calcd for $\mathrm{C}_{14} \mathrm{H}_{18} \mathrm{NO}_{2}{ }^{+}(\mathrm{M}+\mathrm{H})^{+}$ 232.1332 , found 232.1333 .

A mixture of cyclic allylic amine 8 (46.2 $\mathrm{mg}, 0.20 \mathrm{mmol})$, 2-(trimethylsilyl)phenyl triflate (2a) (83.6 mg, $0.28 \mathrm{mmol})$, and $\mathrm{CsF}(60.5 \mathrm{mg}, 0.40 \mathrm{mmol})$ in acetonitrile $(1.0 \mathrm{~mL})$ was heated at $60{ }^{\circ} \mathrm{C}$ (oil bath) for $12 \mathrm{~h}$. The mixture was cooled to room temperature, concentrated, and purified by silica gel chromatography, using a mixture of ethyl acetate and petroleum ether (1:30) as the eluent, to give $\left(1 S^{\prime}, 2 R^{\prime}\right)$-ethyl 1-(diphenylamino)-2-vinylcyclopropanecarboxylate (9) (39.9 $\mathrm{mg}, 65 \%$ yield) as a yellow oil. The relative stereochemistry of cyclopropane 9 was determined by 2D NOESY spectroscopic analysis (see below). ${ }^{1} \mathrm{H}$ NMR $\left(400 \mathrm{MHz}, \mathrm{CDCl}_{3}\right) \delta$ 7.41-7.34 (m, 2H), 7.30-7.23 (m, 2H), 7.23-7.16 (m, 1H), 7.14-7.07 (m, 2H), 6.81-6.72 (m, 3H), 5.61 (ddd, $J=16.8,10.0,9.2 \mathrm{~Hz}, 1 \mathrm{H})$, $5.31(\mathrm{dd}, J=16.8,1.2 \mathrm{~Hz}, 1 \mathrm{H}), 5.07$ (dd, $J=10.0,1.2 \mathrm{~Hz}, 1 \mathrm{H}), 4.20-4.05$ (m, 2H), 2.95-2.87 (m, $1 \mathrm{H}), 1.71(\mathrm{dd}, J=9.2,5.2 \mathrm{~Hz}, 1 \mathrm{H}), 1.12-1.00(\mathrm{~m}, 4 \mathrm{H}) ;{ }^{13} \mathrm{C} \mathrm{NMR}\left(100 \mathrm{MHz}, \mathrm{CDCl}_{3}\right) \delta 173.5,147.9$, $145.5,135.2$, 129.6, 128.6, 127.5, 125.3, 118.9, 117.3, 115.6, 61.5, 48.8, 36.0, 24.7, 14.2; HRMS (ESI) calcd for $\mathrm{C}_{20} \mathrm{H}_{22} \mathrm{NO}_{2}{ }^{+}(\mathrm{M}+\mathrm{H})^{+} 308.1465$, found 308.1467.

(5) Synthesis of Indole $\mathbf{1 1}^{13}$

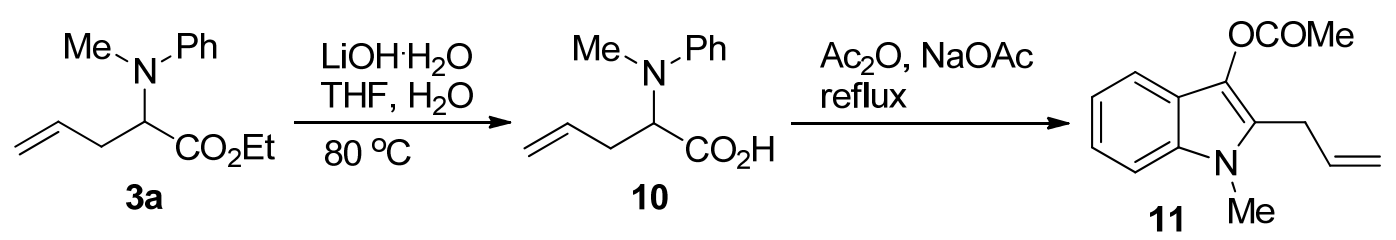

To a solution of ester 3a $(117 \mathrm{mg}, 0.50 \mathrm{mmol})$ in tetrahydrofuran $(1.0 \mathrm{~mL})$ were added water $(1.0 \mathrm{~mL})$ and $\mathrm{LiOH} \cdot \mathrm{H}_{2} \mathrm{O}(105 \mathrm{mg}, 2.5 \mathrm{mmol})$. The mixture was heated at $80{ }^{\circ} \mathrm{C}$ for $8 \mathrm{~h}$, cooled to room temperature, and added hydrochloric acid $(1.0 \mathrm{M})$ until $\mathrm{pH}=7.0$. The mixture was extracted with ethyl acetate $(3 \times 5 \mathrm{~mL})$, dried over anhydrous sodium sulfate, and concentrated to give crude acid 10. Crude acid 10 was transferred into a $10 \mathrm{~mL}$ round-bottom flask, to which were added sodium acetate $(410 \mathrm{mg}, 5.0 \mathrm{mmol})$ and acetic anhydride $(2.0 \mathrm{~mL})$. The mixture was heated at 120 ${ }^{\circ} \mathrm{C}$ for $5 \mathrm{~h}$. The residue was purified by silica gel chromatography, using a mixture of ethyl acetate and petroleum ether (1:10) as the eluent, to give 2-allyl-1-methyl- $1 H$-indol-3-yl acetate (11) (38.9 $\mathrm{mg}, 34 \%$ yield $)$ as a brown oil. ${ }^{1} \mathrm{H} \mathrm{NMR}\left(400 \mathrm{MHz}, \mathrm{CDCl}_{3}\right) \delta 7.36(\mathrm{~d}, J=8.0 \mathrm{~Hz}, 1 \mathrm{H}), 7.29-7.24(\mathrm{~m}$, $1 \mathrm{H}), 7.21-7.15(\mathrm{~m}, 1 \mathrm{H}), 7.11-7.06(\mathrm{~m}, 1 \mathrm{H}), 5.96-5.85(\mathrm{~m}, 1 \mathrm{H}), 5.14-5.02(\mathrm{~m}, 2 \mathrm{H}), 3.64(\mathrm{~s}, 3 \mathrm{H}), 3.46$ $(\mathrm{dt}, J=5.6,1.6 \mathrm{~Hz}, 2 \mathrm{H}), 2.36(\mathrm{~s}, 3 \mathrm{H}) ;{ }^{13} \mathrm{C} \mathrm{NMR}\left(100 \mathrm{MHz}, \mathrm{CDCl}_{3}\right) \delta 169.8,134.5,134.1,126.9$, 126.6, 121.7, 120.5, 119.7, 117.1, 116.8, 109.2, 29.8, 28.1, 20.7; HRMS (ESI) calcd for $\mathrm{C}_{14} \mathrm{H}_{16} \mathrm{NO}_{2}{ }^{+}$ $(\mathrm{M}+\mathrm{H})^{+} 230.1776$, found 230.1775 . 


\section{References}

(1) Doyle, L.; Heaney, F. Tetrahedron 2010, 66, 7041-7049.

(2) Hassner, A.; Maurya, R.; Friedman, O.; Gottlieb, H. E. J. Org. Chem. 1993, 58, 4539-4546.

(3) Sun, M.-R.; Lu, H.-T.; Wang, Y.-Z.; Yang, H.; Liu, H.-M. J. Org. Chem. 2009, 74, 2213-2216.

(4) Shaibu, B. S.; Kawade, R. K.; Liu, R.-S. Org. Biomol. Chem. 2012, 10, 6834-6839.

(5) Kong, H. I.; Crichton, J. E.; Manthorpe, J. M. Tetrahedron Lett. 2011, 52, 3714-3717.

(6) Hopkins, B. A.; Wolfe, J. P. Angew. Chem. Int. Ed. 2012, 51, 9886-9890.

(7) Shinada, T.; Oe, K.; Ohfune, Y. Tetrahedron Lett. 2012, 53, 3250-3253.

(8) Nakajima, M.; Oda, Y.; Wada, T.; Minamikawa, R.; Shirokane, K.; Sato, T.; Chida, N. Chem. Eur. J. 2014, 20, 17565-17571.

(9) Prishchenko, A. A.; Livantsov, M. V.; Novikova, O. P.; Livantsova, L. I.; Petrosyan, V. S. Heteroatom Chem. 2010, 21, 430-440.

(10) Zhou, C.-Y.; Yu, W.-Y.; Chan, P. W. H.; Che, C.-M. J. Org. Chem. 2004, 69, 7072-7082.

(11) Chen, C.-C.; Chen, S.-T.; Chuang, T.-H.; Fang, J.-M. J. Chem. Soc., Perkin Trans. 1 1994, 2217-2221.

(12) West, T. H.; Daniels, D. S. B.; Slawin, A. M. Z.; Smith, A. D. J. Am. Chem. Soc. 2014, 136, 4476-4479.

(13) Brady, W. T.; Gu, Y. Q. J. Org. Chem. 1989, 54, 2834-2838. 


$$
\sim^{\mathrm{Me}} \mathrm{CO}_{2} \mathrm{Et}
$$

$1 a$

${ }^{1} \mathrm{H} \mathrm{NMR}\left(400 \mathrm{MHz}, \mathrm{CDCl}_{3}\right)$

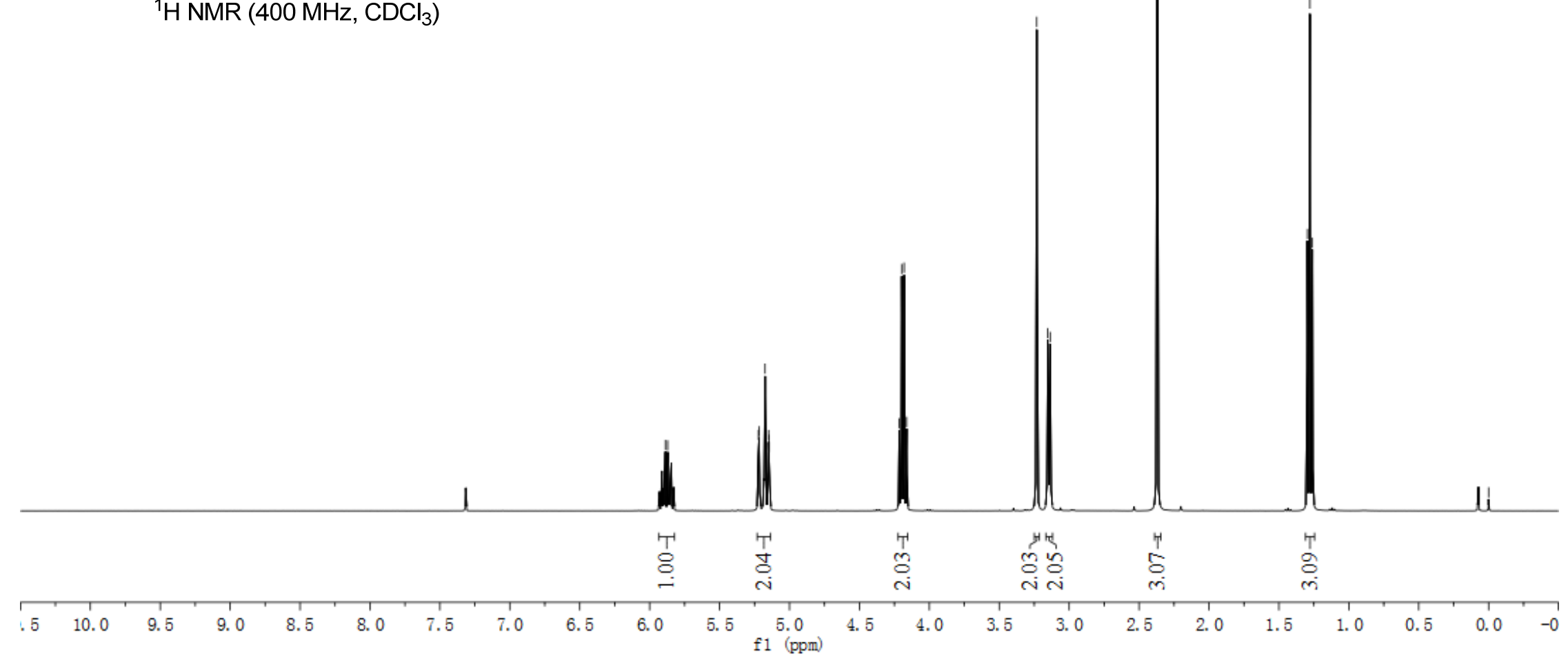




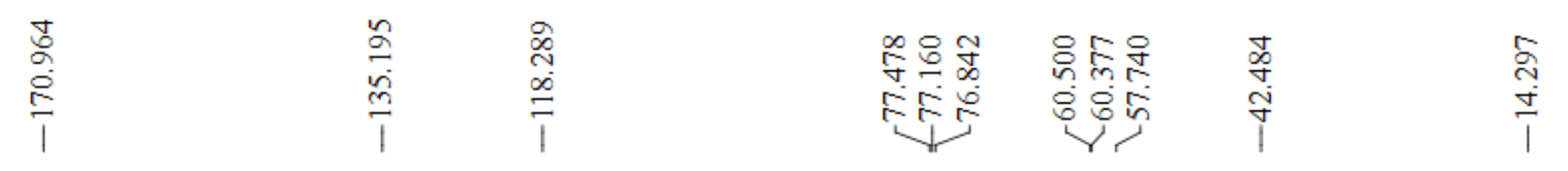

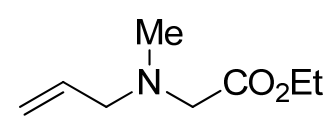

$1 \mathbf{a}$

${ }^{13} \mathrm{C}$ NMR $\left(100 \mathrm{MHz}, \mathrm{CDCl}_{3}\right)$

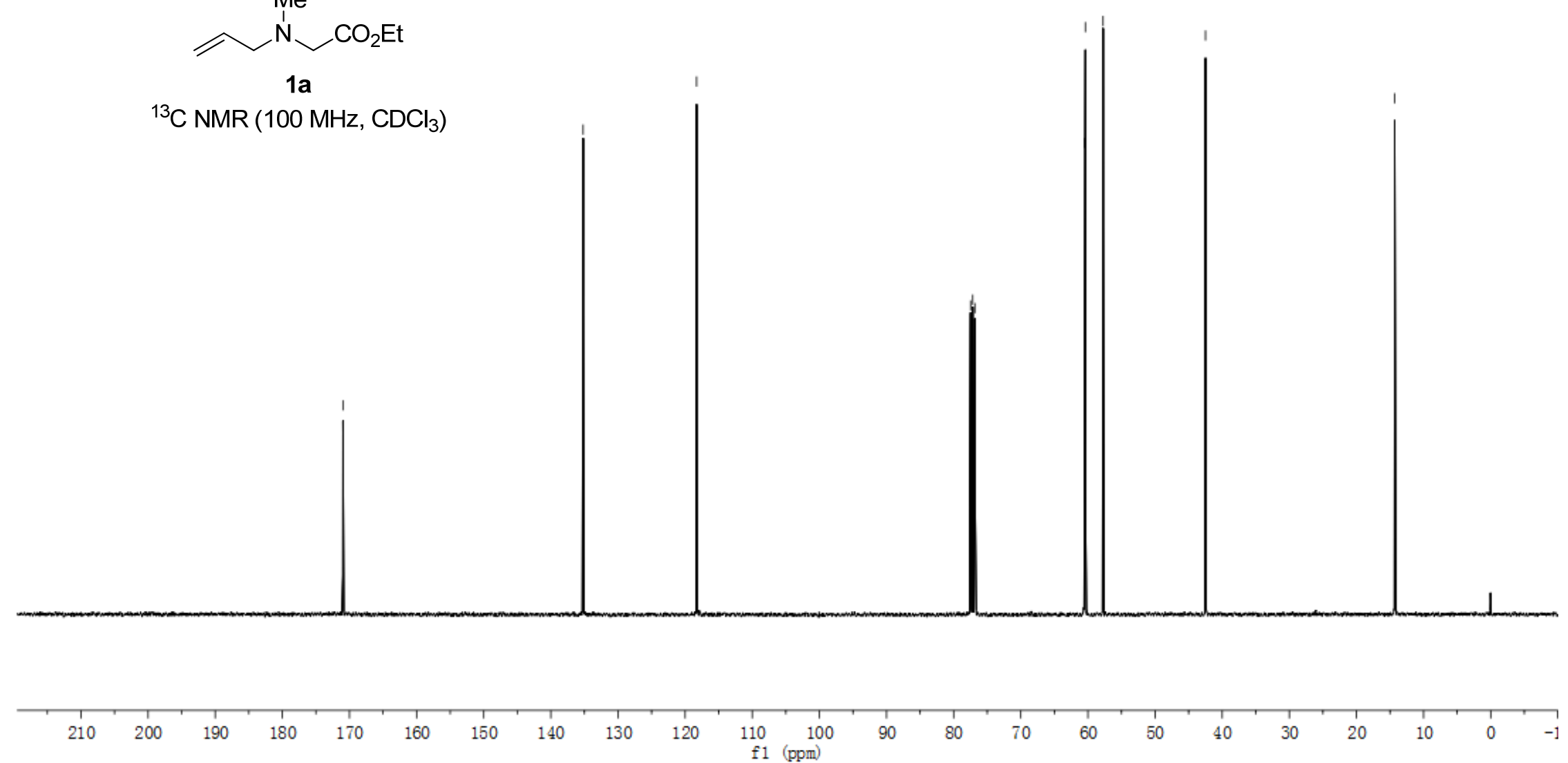

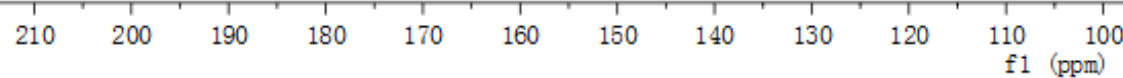

S-26 


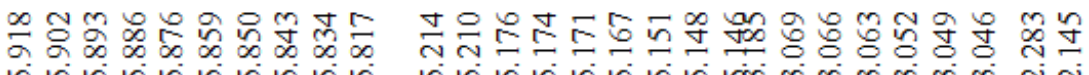

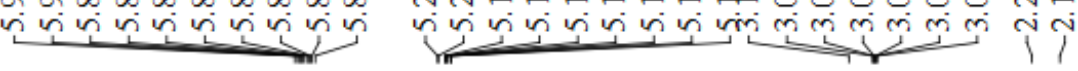

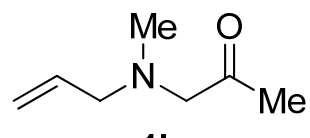

1b

${ }^{1} \mathrm{H} \mathrm{NMR}\left(400 \mathrm{MHz}, \mathrm{CDCl}_{3}\right)$

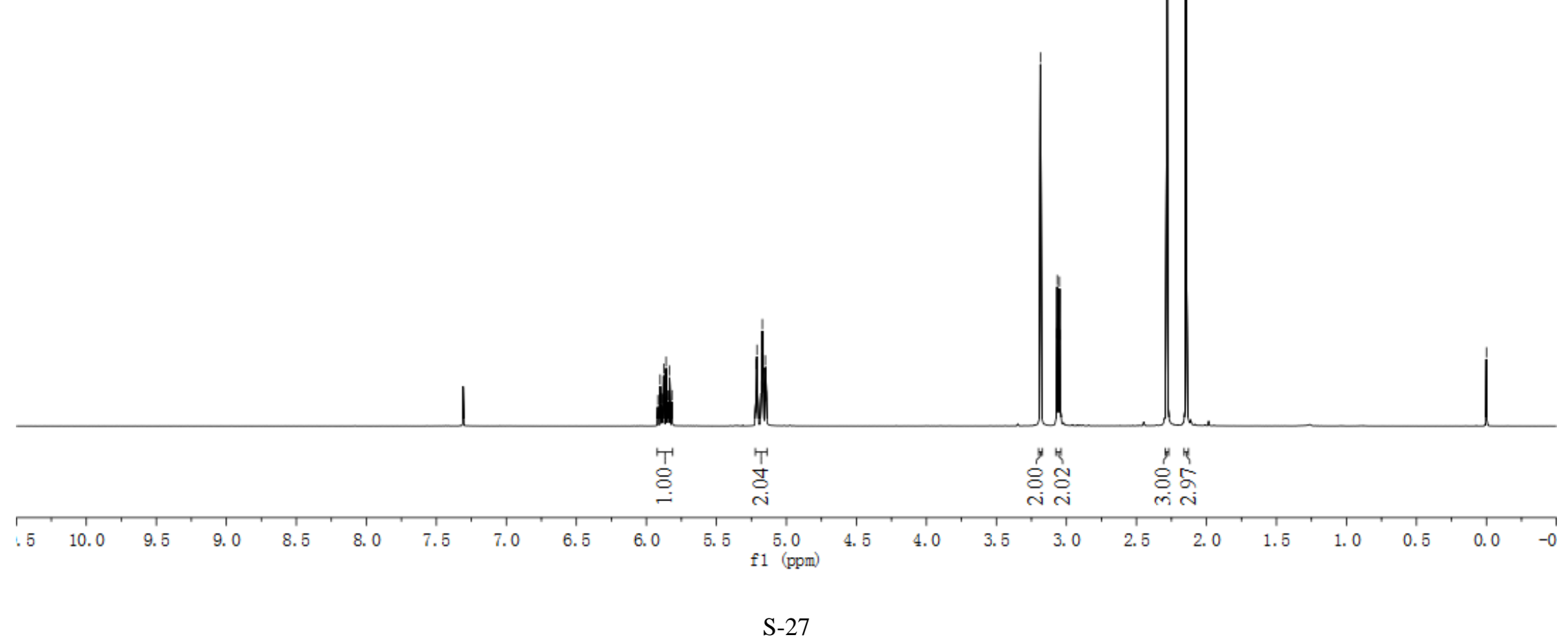




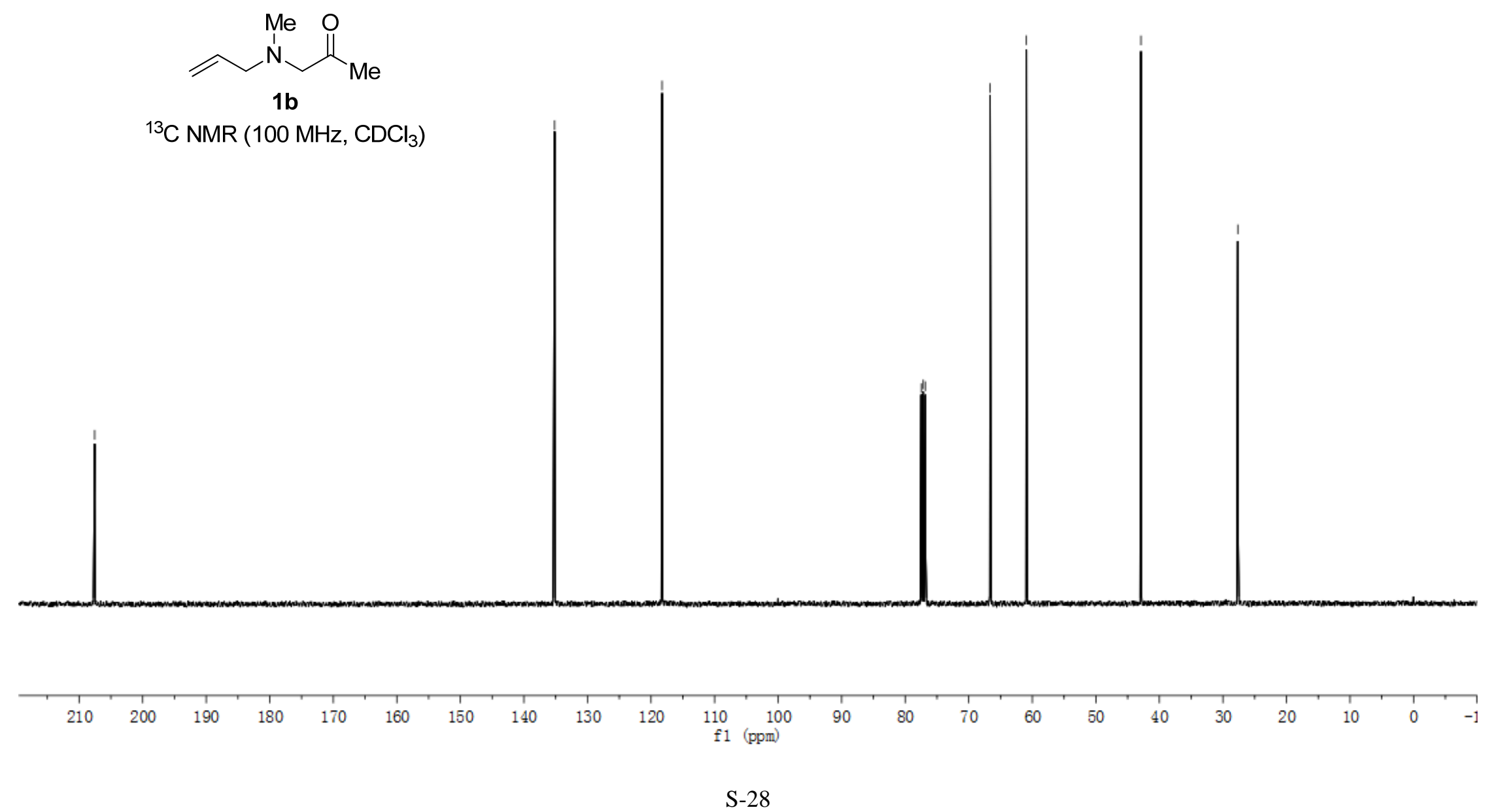




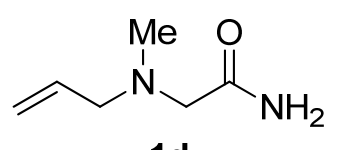

${ }^{1} \mathrm{H} \mathrm{NMR}\left(400 \mathrm{MHz}, \mathrm{CDCl}_{3}\right)$

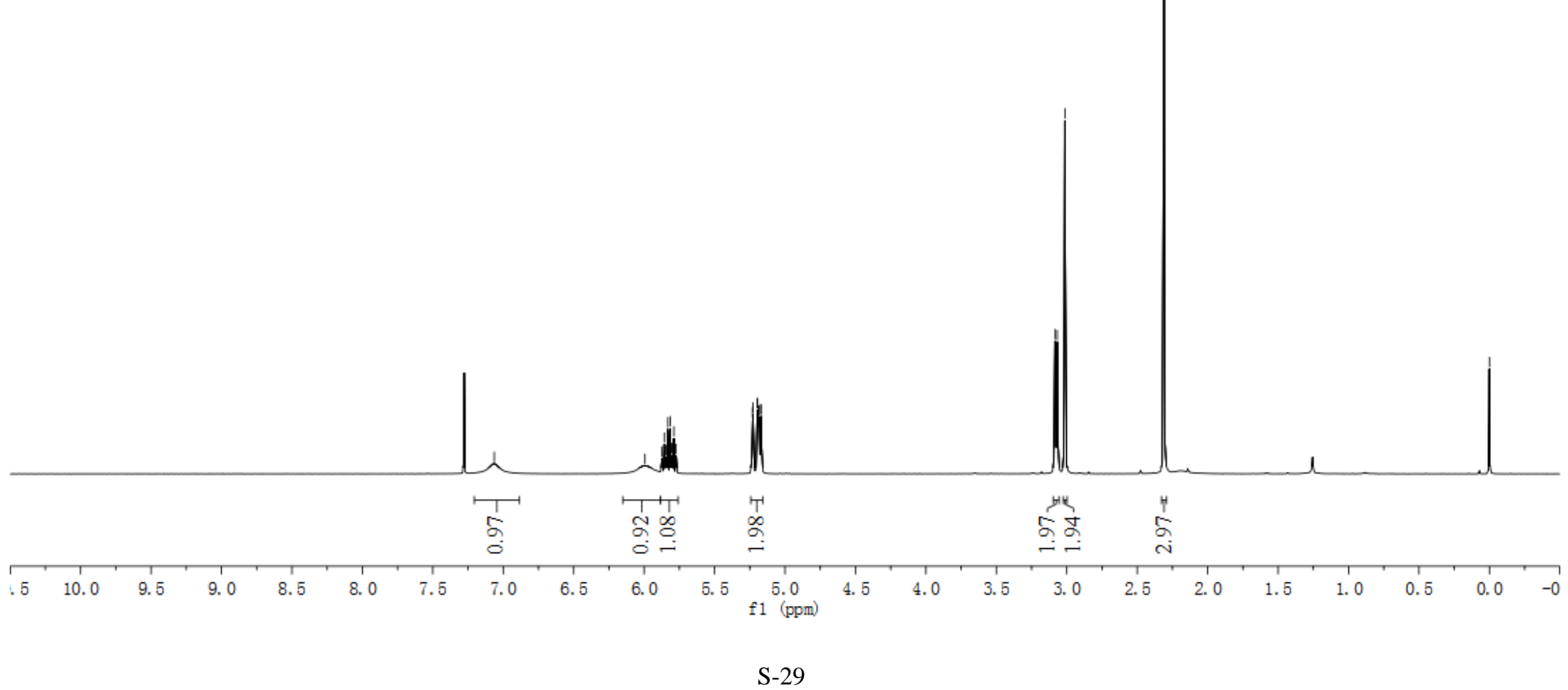




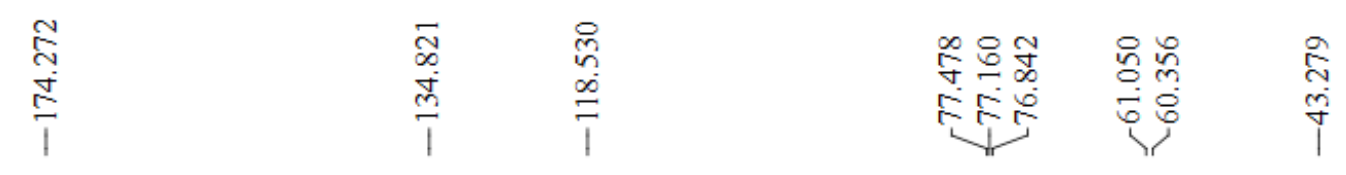

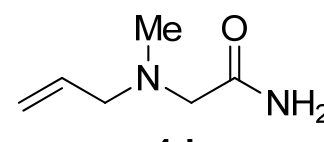

1d

${ }^{13} \mathrm{C}$ NMR $\left(100 \mathrm{MHz}, \mathrm{CDCl}_{3}\right)$

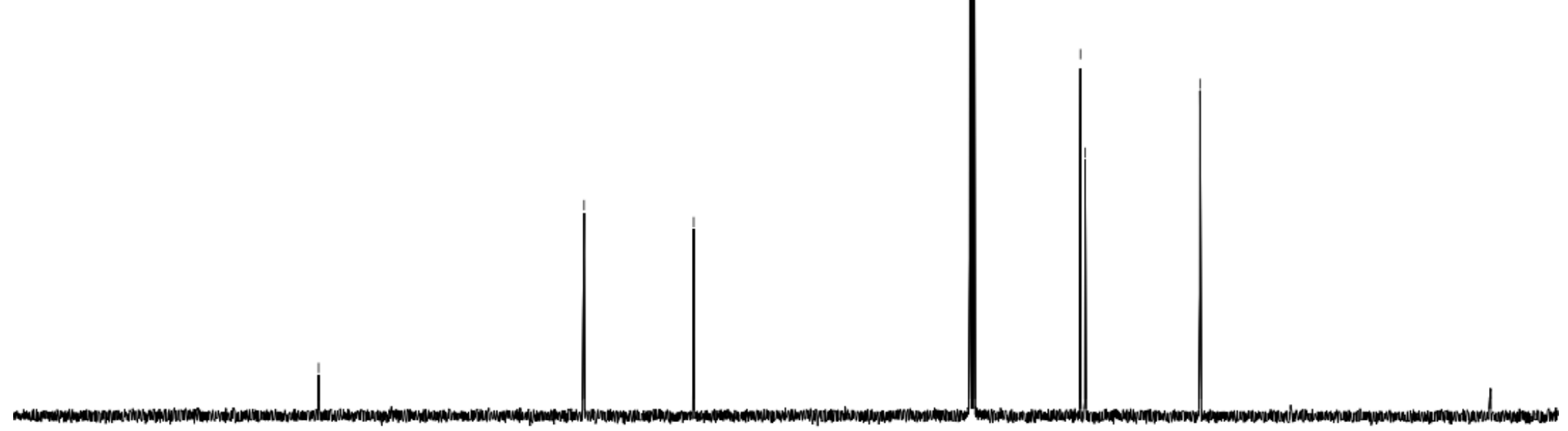

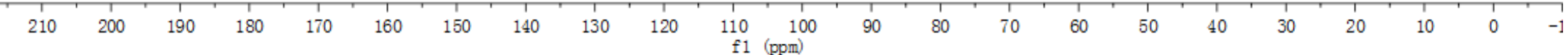

S-30 


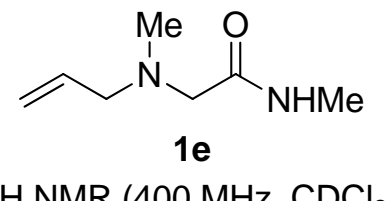

${ }^{1} \mathrm{H}$ NMR $\left(400 \mathrm{MHz}, \mathrm{CDCl}_{3}\right)$

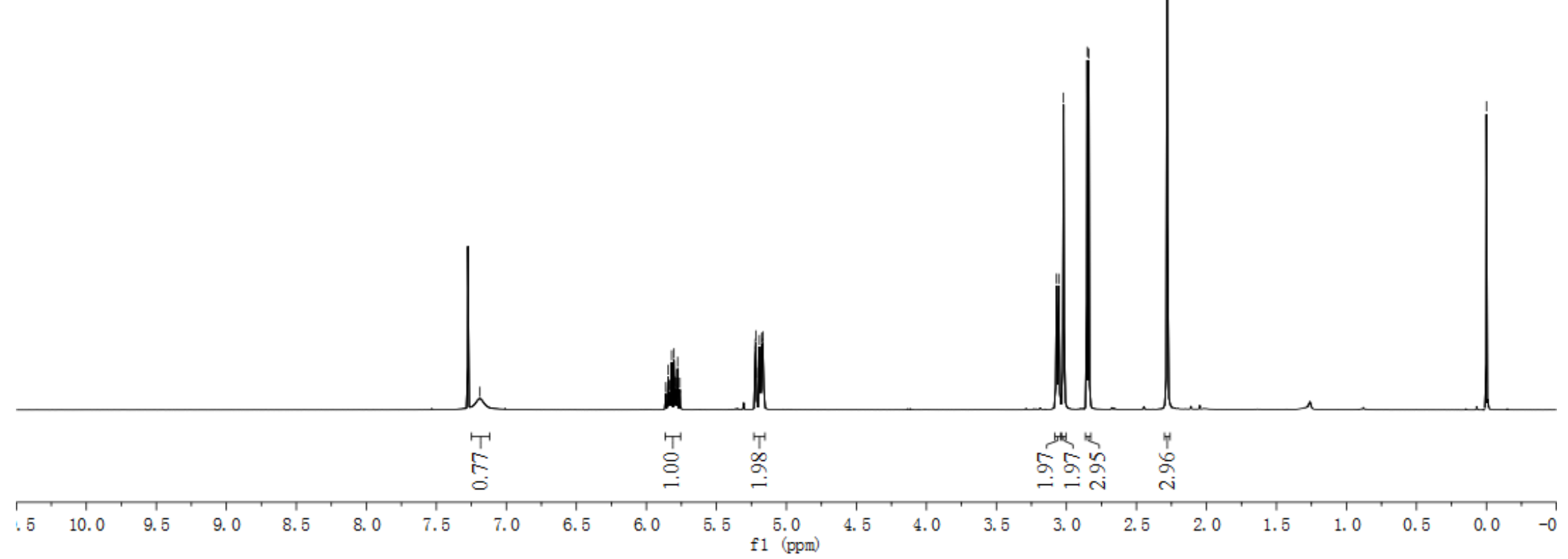



$\underset{\substack{\infty \\:}}{\stackrel{\infty}{=}}$

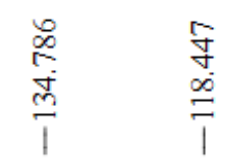

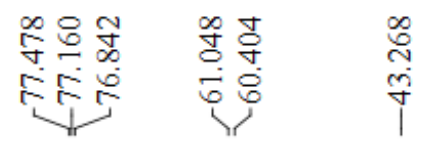
$\underset{\substack{0 \\ 0}}{\substack{0 \\ i}}$

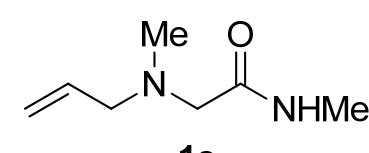

$1 e$

${ }^{13} \mathrm{C} \mathrm{NMR}\left(100 \mathrm{MHz}, \mathrm{CDCl}_{3}\right)$

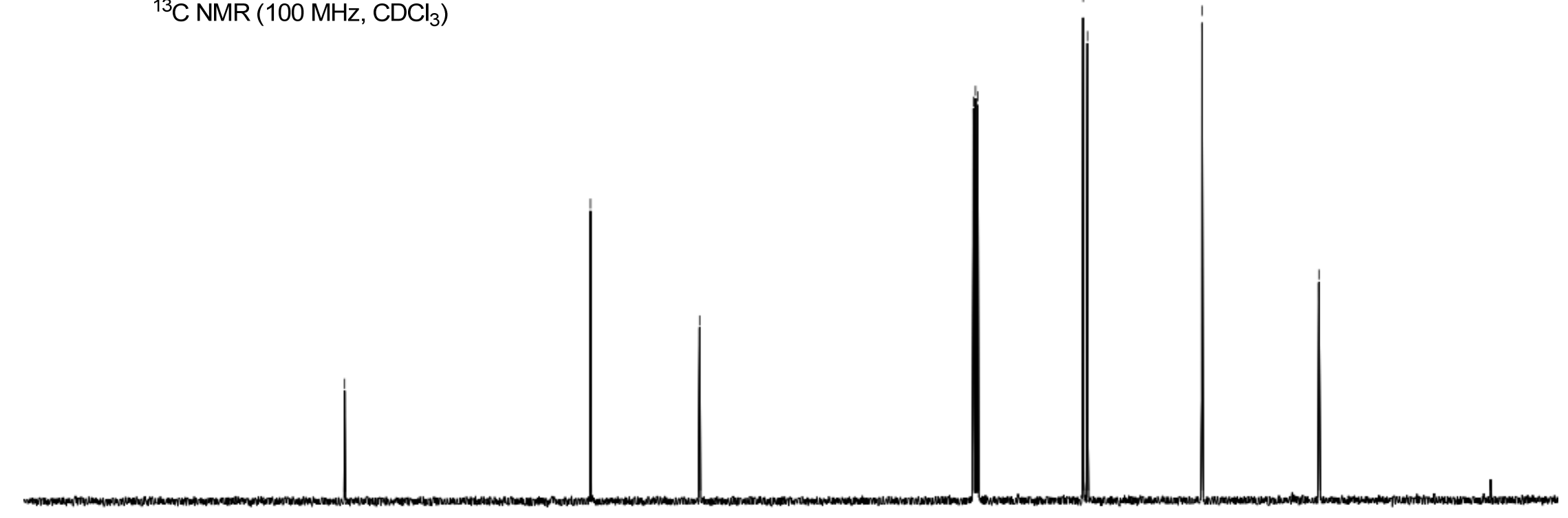

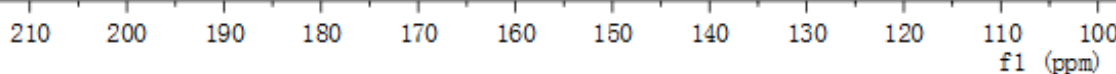




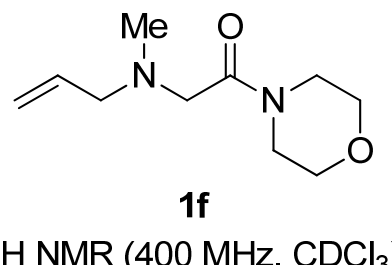

${ }^{1} \mathrm{H}$ NMR (400 MHz, $\left.\mathrm{CDCl}_{3}\right)$

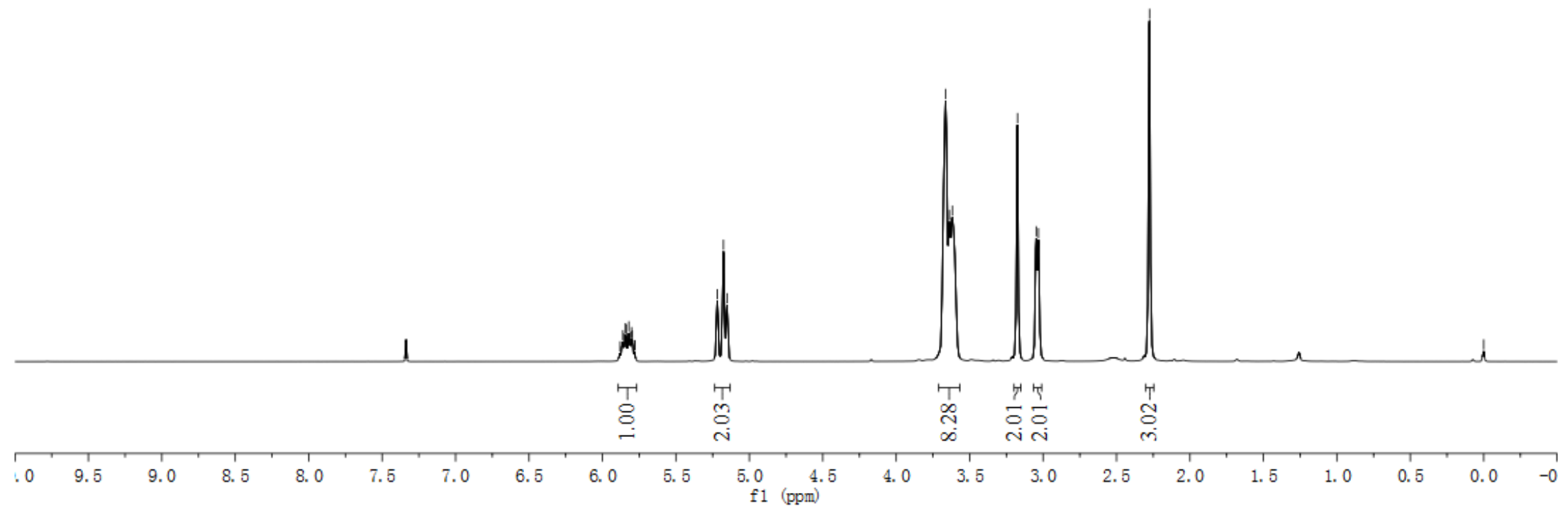




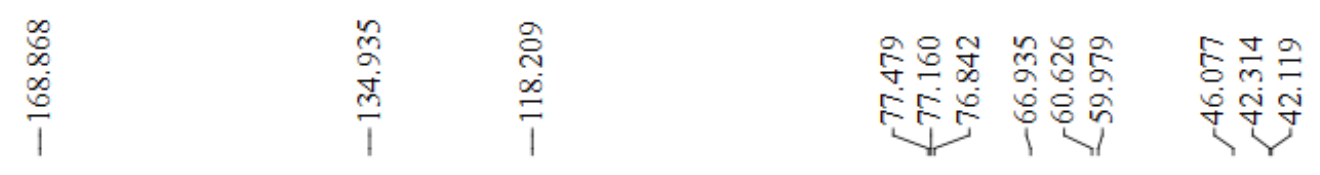
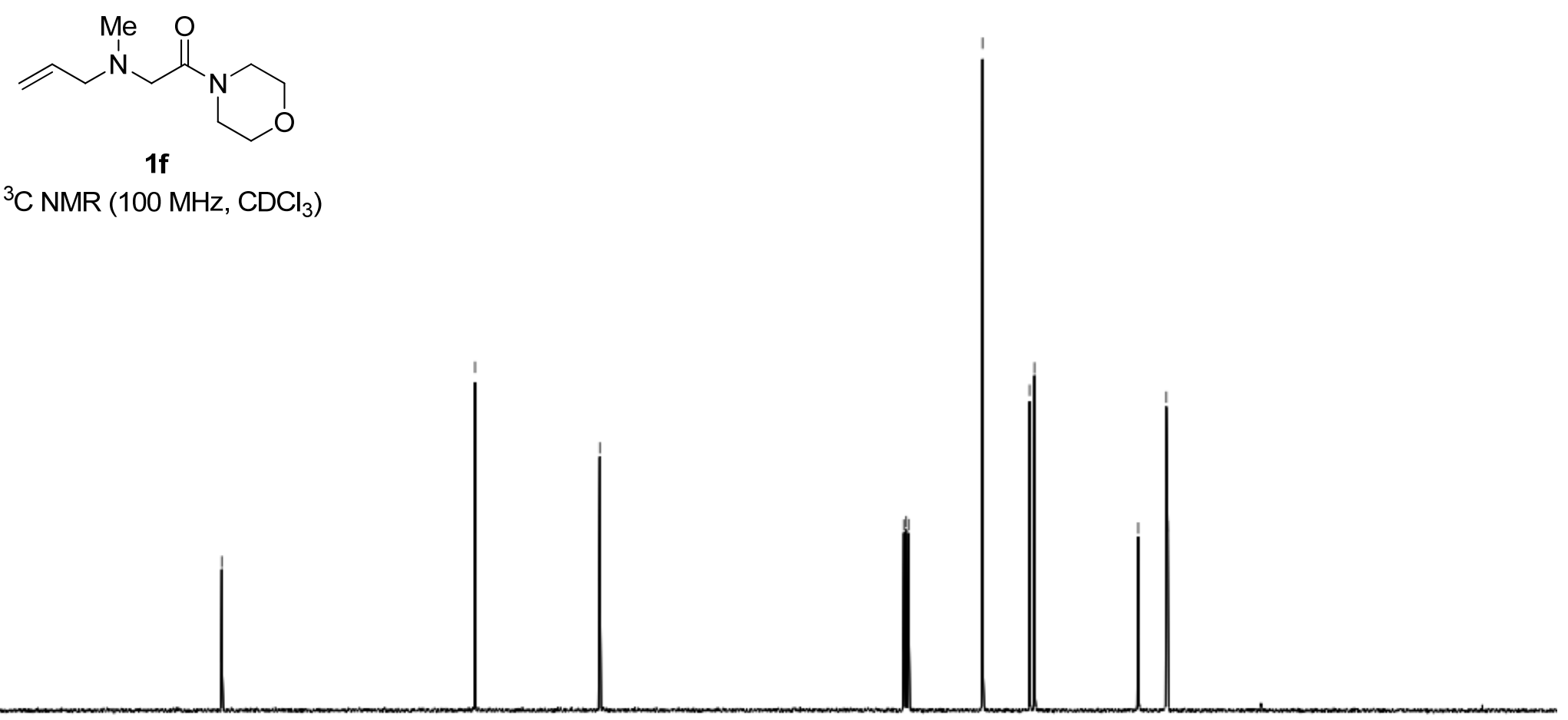

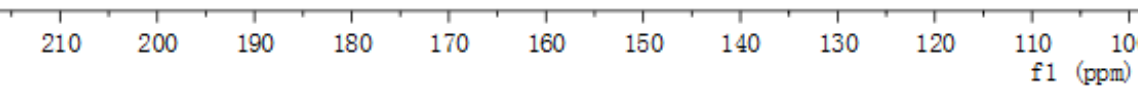




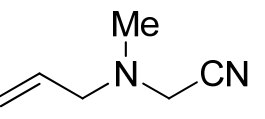

\section{$1 \mathrm{~g}$}

${ }^{1} \mathrm{H} \mathrm{NMR}\left(400 \mathrm{MHz}, \mathrm{CDCl}_{3}\right)$

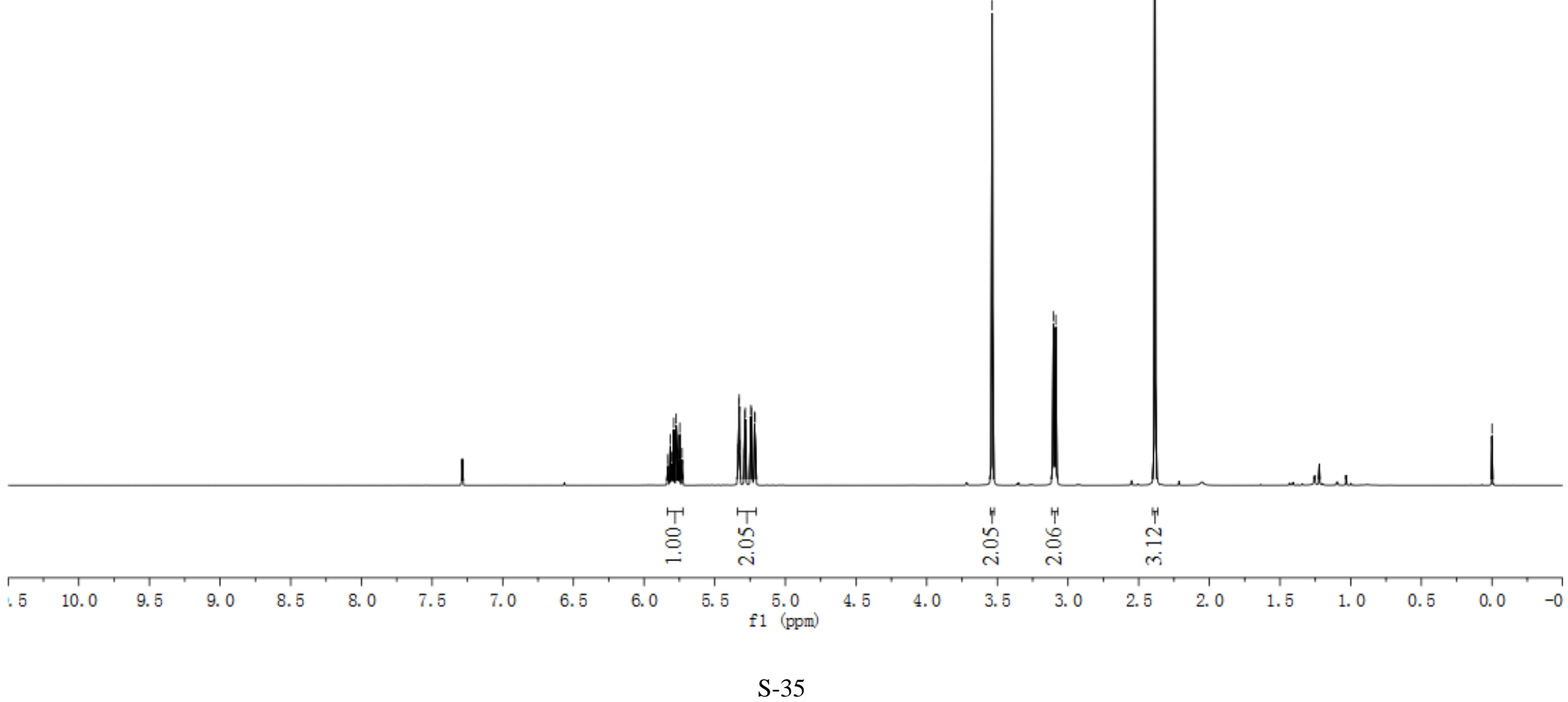




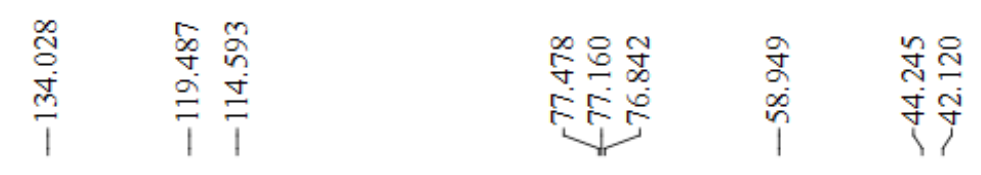

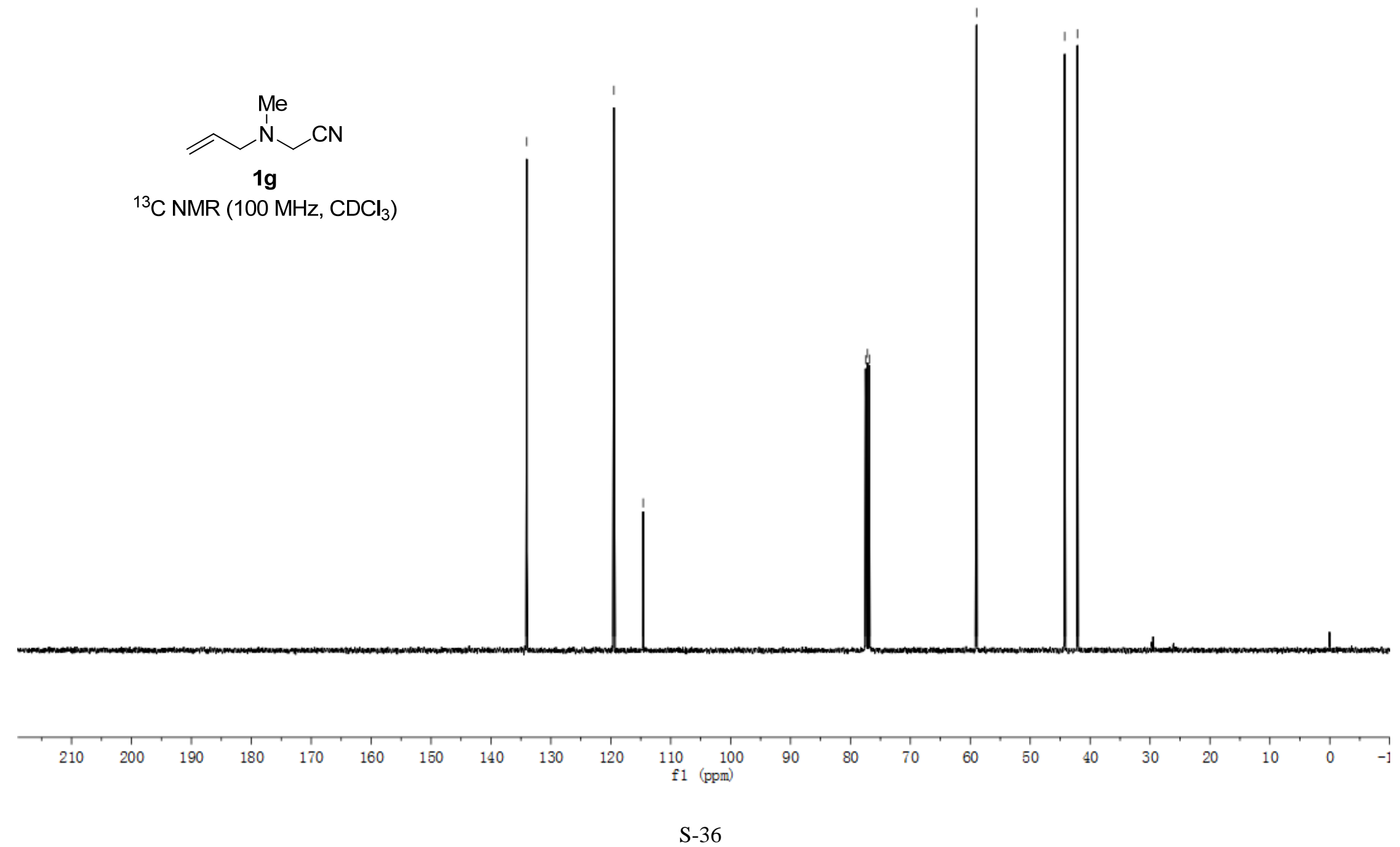




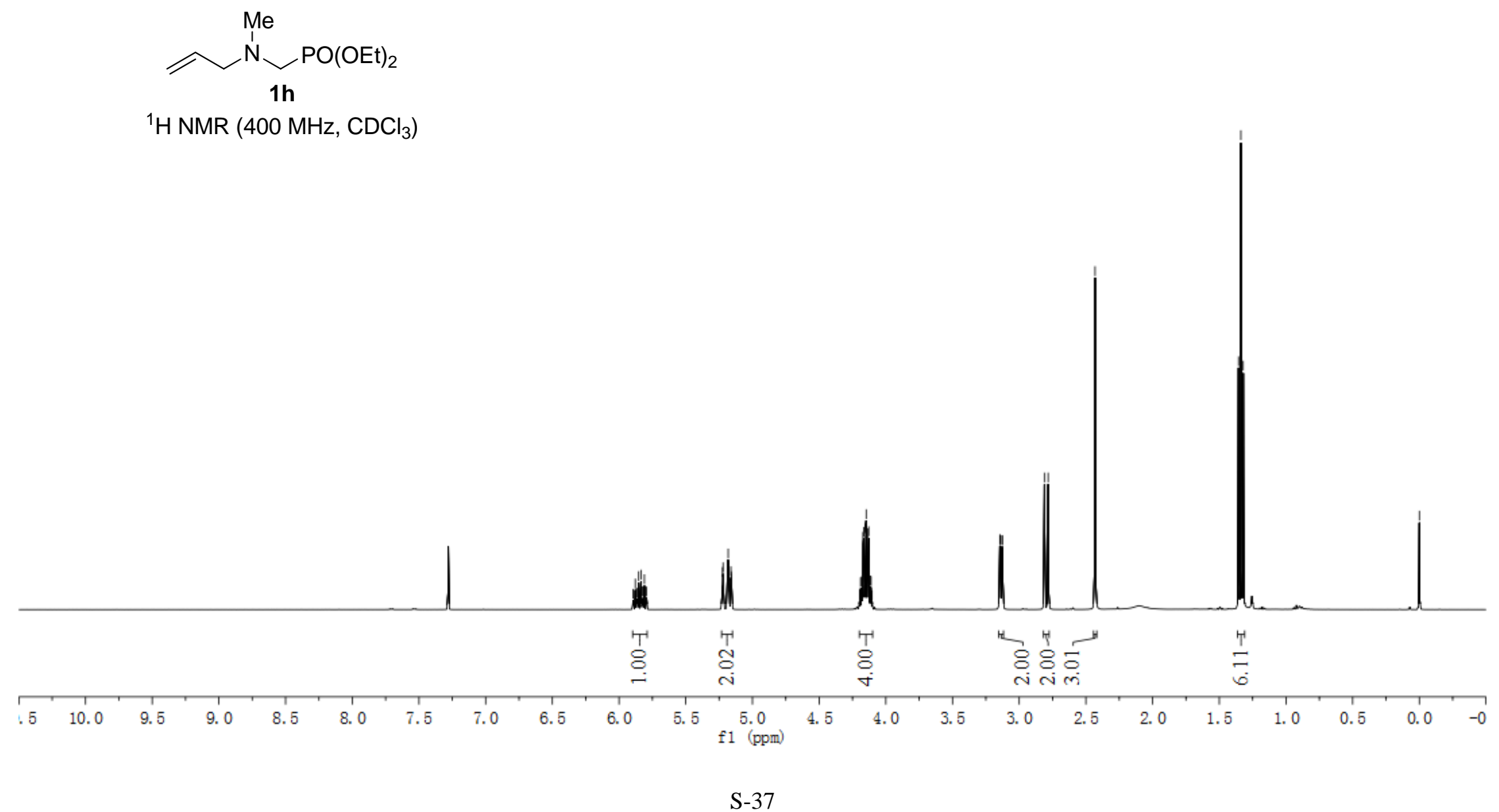




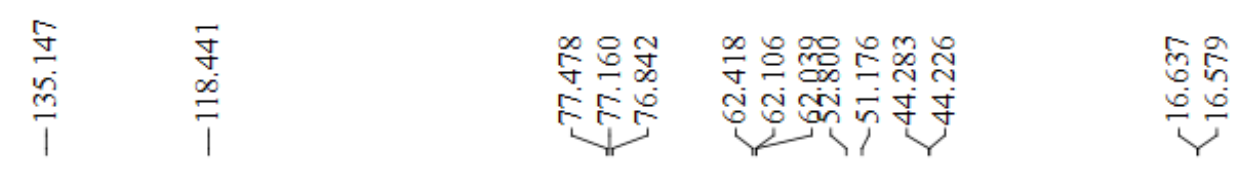

$\sim_{1 \mathrm{~h}}^{\mathrm{Me}} \mathrm{PO}(\mathrm{OEt})_{2}$

${ }^{13} \mathrm{C} \mathrm{NMR}\left(100 \mathrm{MHz}, \mathrm{CDCl}_{3}\right)$

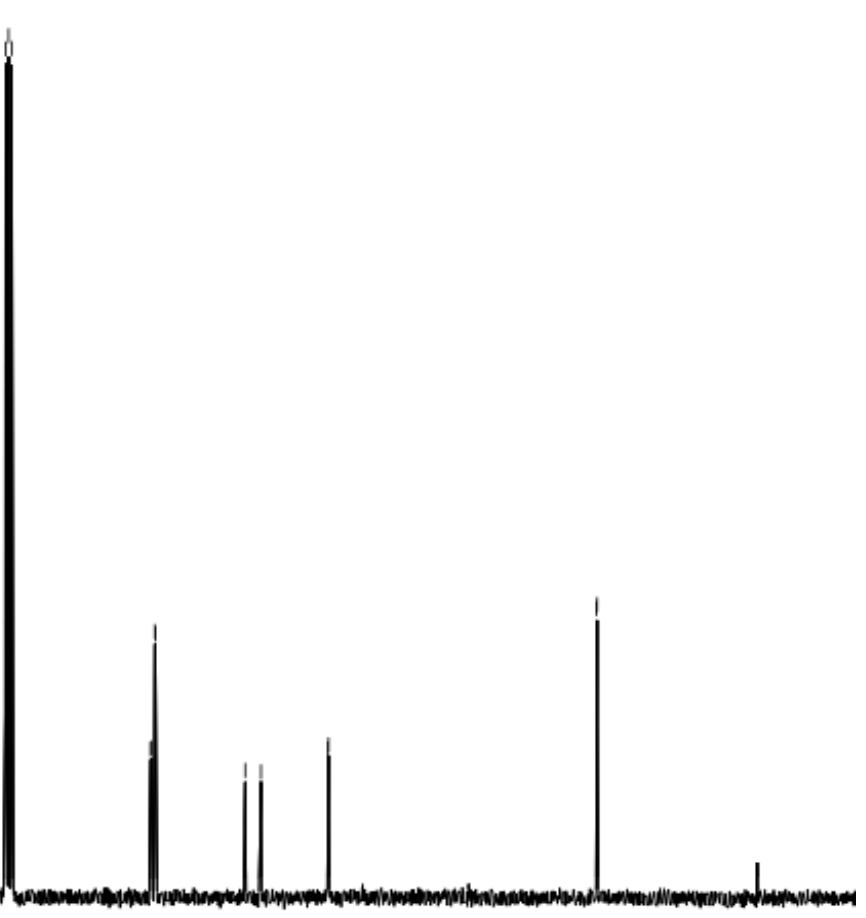

$\begin{array}{llllllllllll}210 & 200 & 190 & 180 & 170 & 160 & 150 & 140 & 130 & 120 & 110 & 100 \\ & & & & & & & & & & & \end{array}$ 


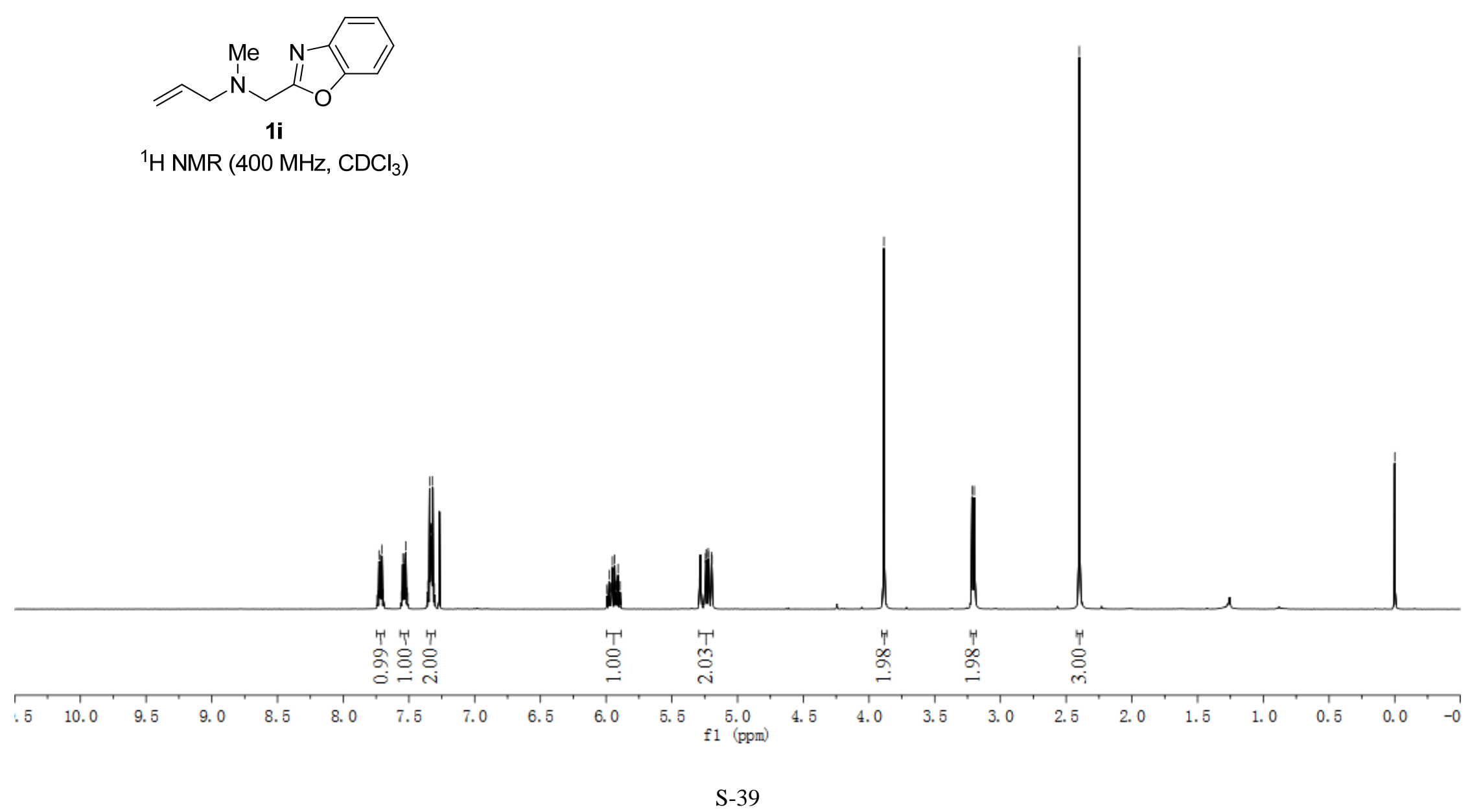




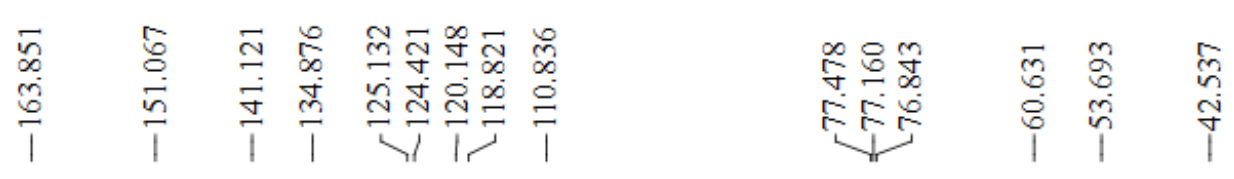

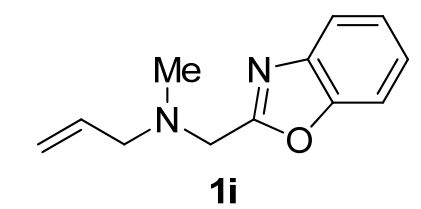

${ }^{13} \mathrm{C} \mathrm{NMR}\left(100 \mathrm{MHz}, \mathrm{CDCl}_{3}\right)$

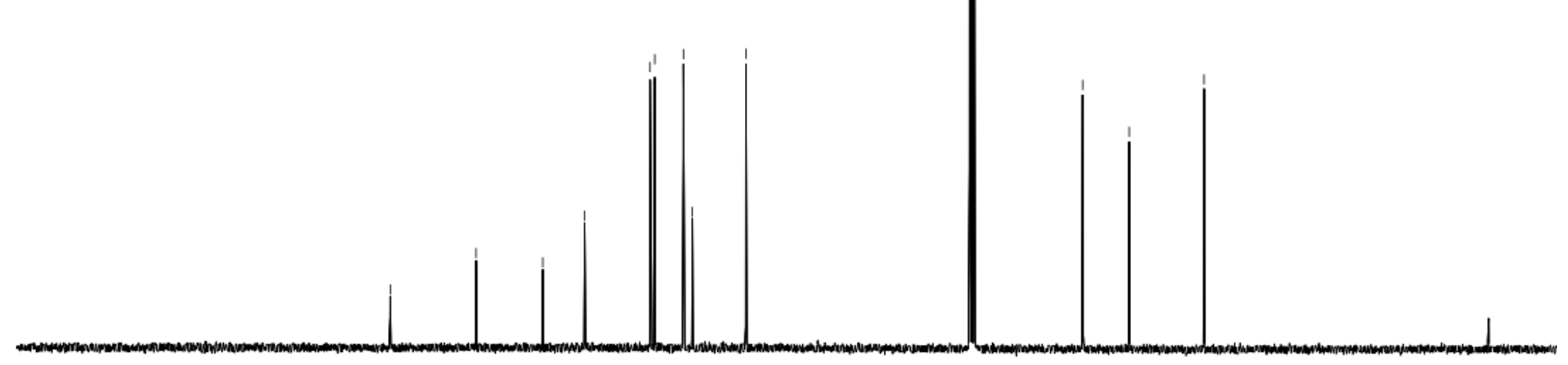

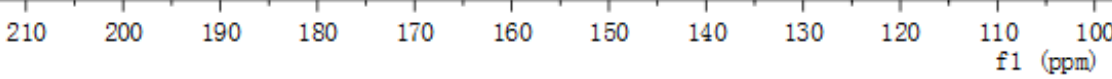




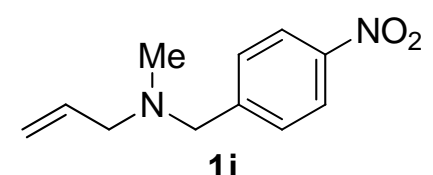

${ }^{1} \mathrm{H}$ NMR $\left(400 \mathrm{MHz}, \mathrm{CDCl}_{3}\right)$

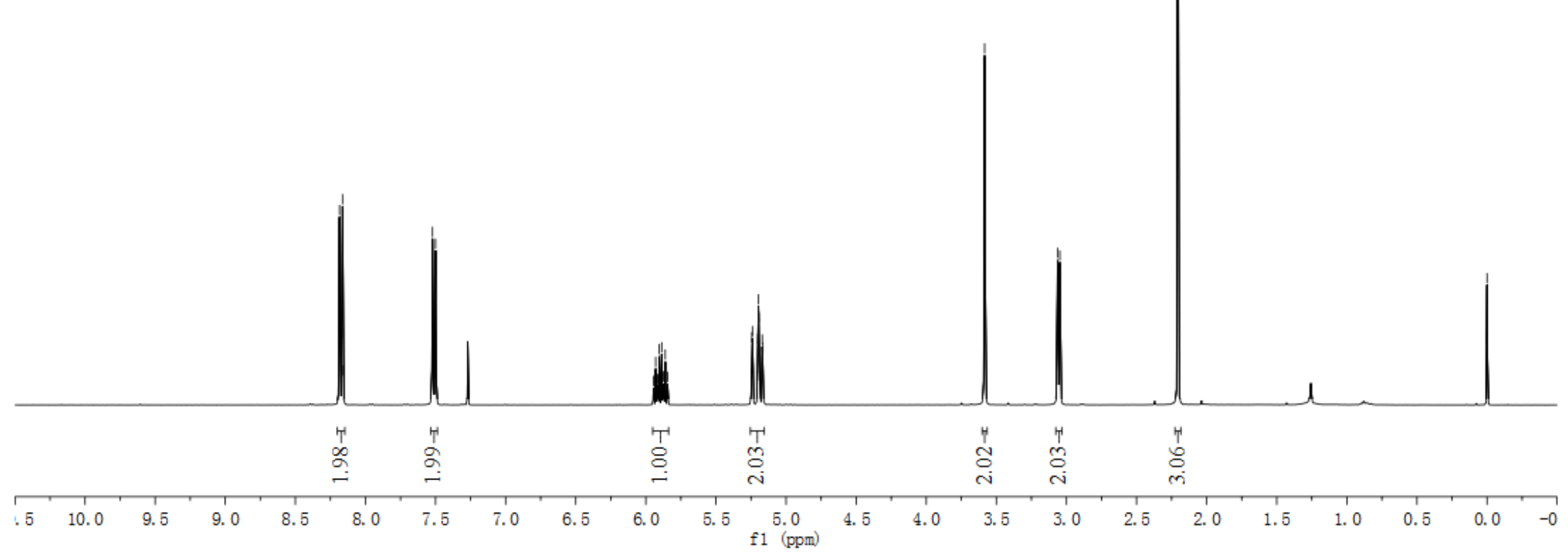




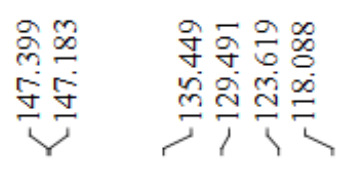

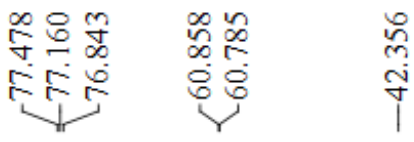

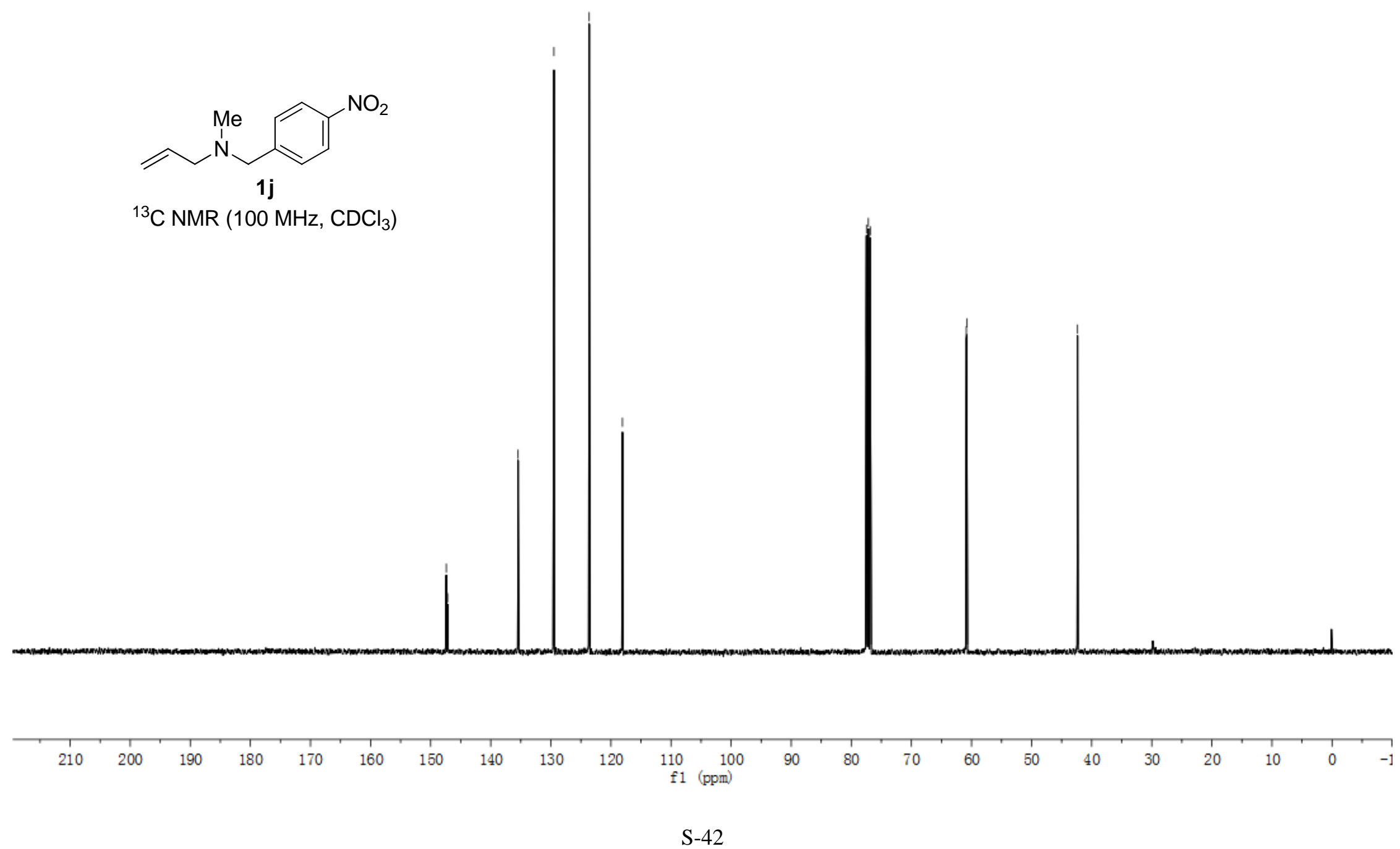




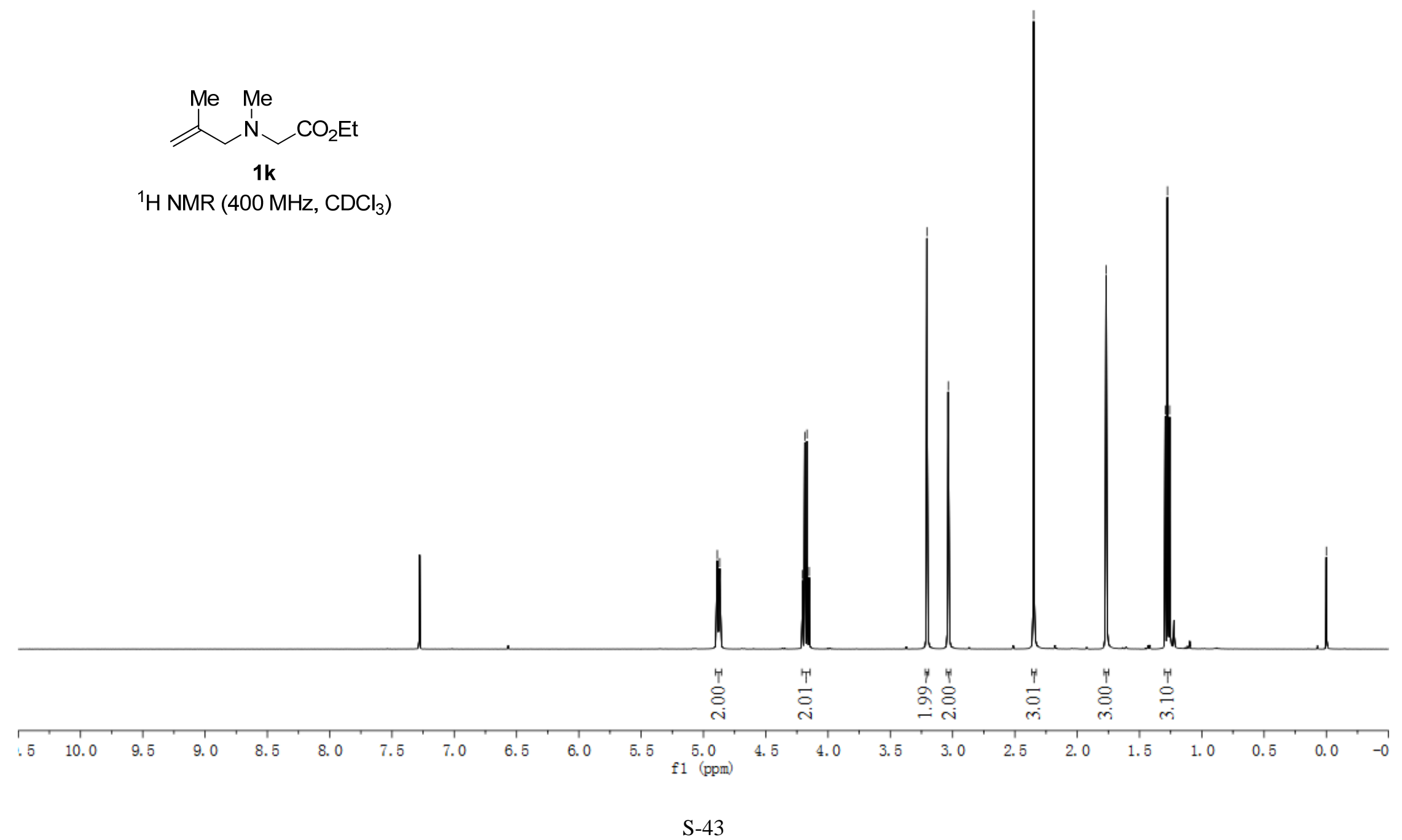




\begin{tabular}{|c|c|c|c|c|}
\hline $\begin{array}{l}\tilde{8} \\
\stackrel{8}{*} \\
\end{array}$ & $\begin{array}{l}\frac{8}{0} \\
\stackrel{0}{0} \\
\stackrel{1}{*}\end{array}$ & 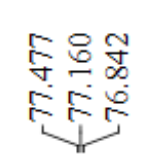 & 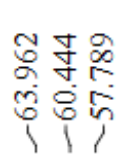 & $\not$ \\
\hline
\end{tabular}

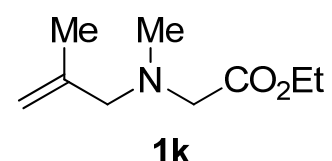

${ }^{13} \mathrm{C} \mathrm{NMR}\left(100 \mathrm{MHz}, \mathrm{CDCl}_{3}\right)$

$\begin{array}{lllllllllll}210 & 200 & 190 & 180 & 170 & 160 & 150 & 140 & 130 & 120 & 110 \\ f 1 & (\mathrm{ppm})\end{array}$ 


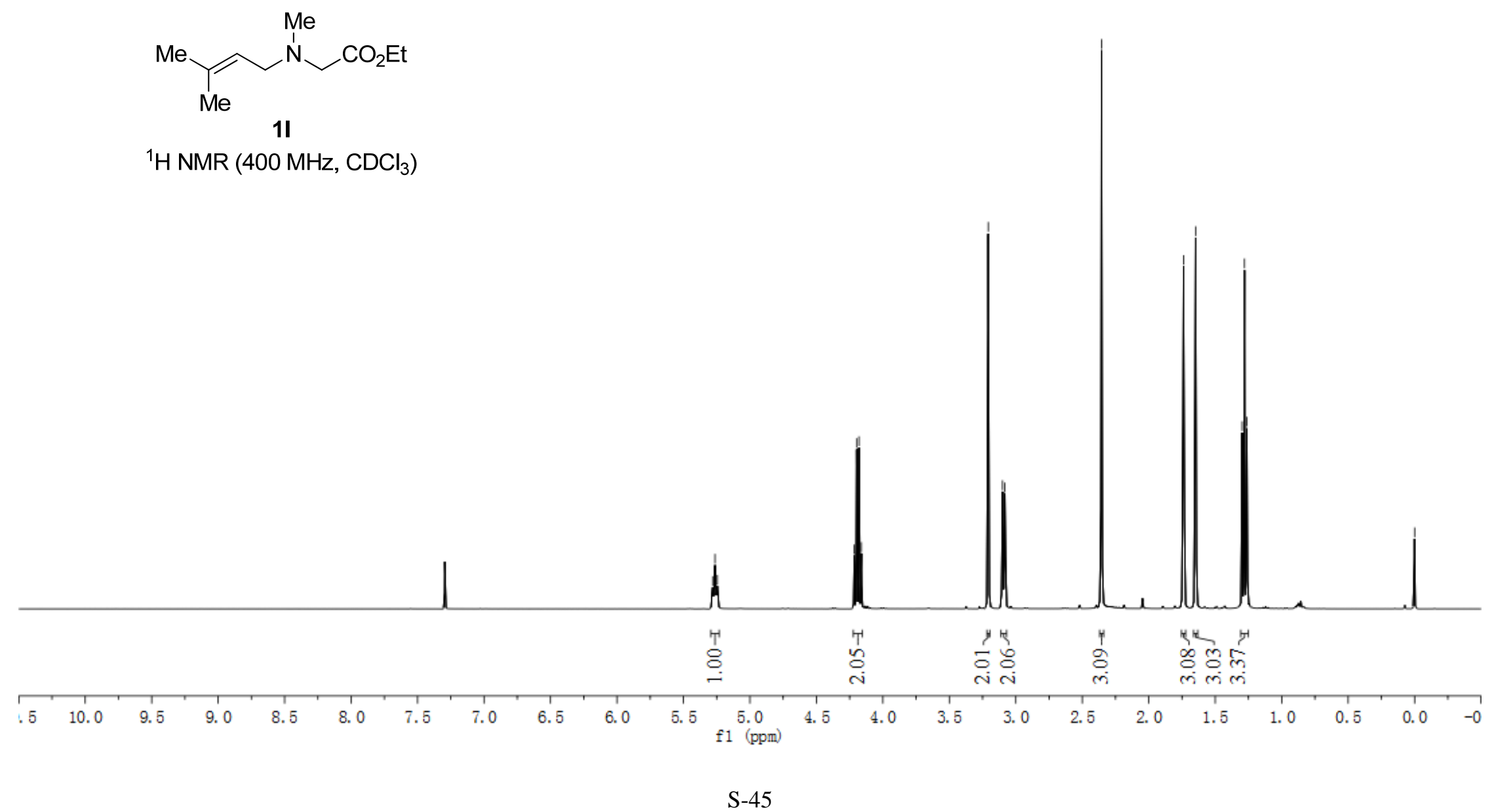




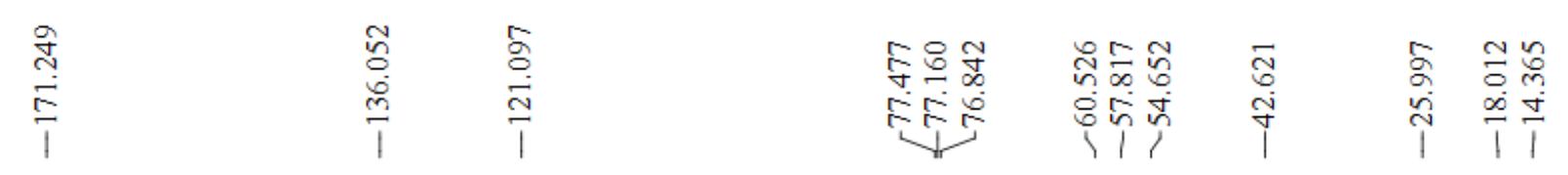

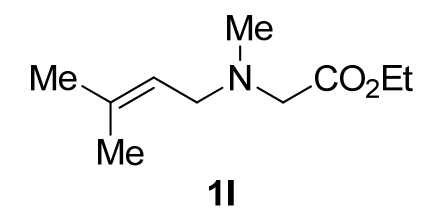

${ }^{13} \mathrm{C}$ NMR $\left(100 \mathrm{MHz}, \mathrm{CDCl}_{3}\right)$

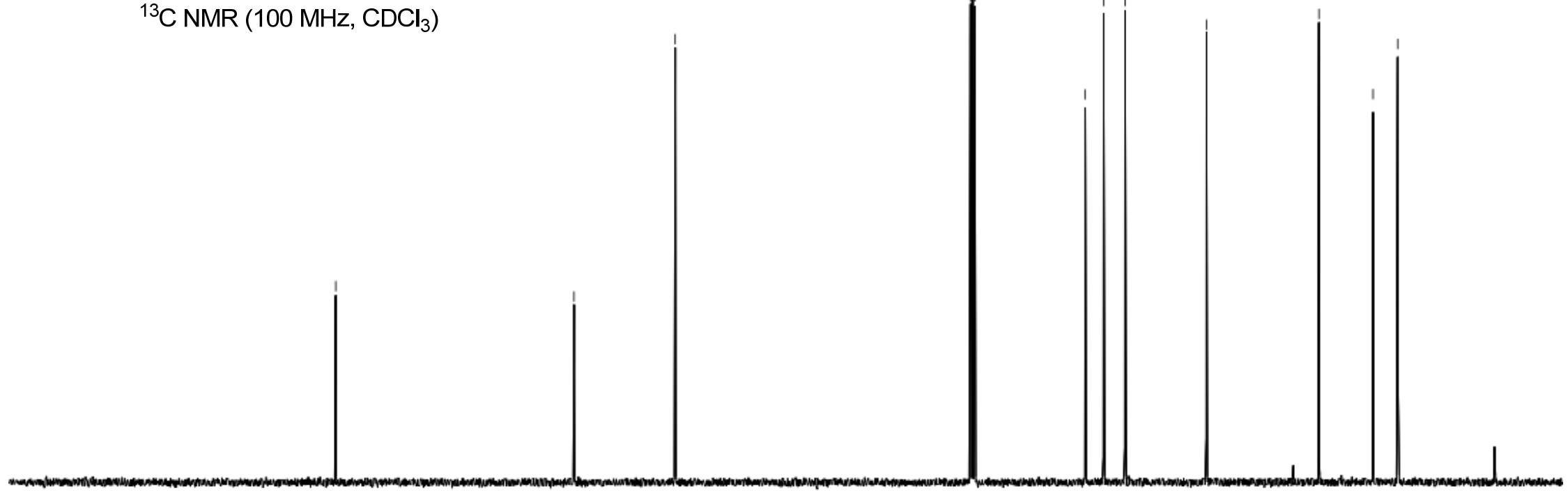

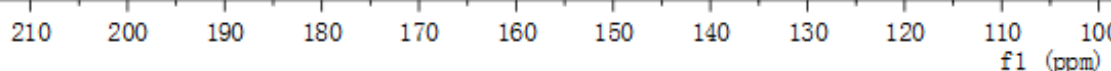

S-46 


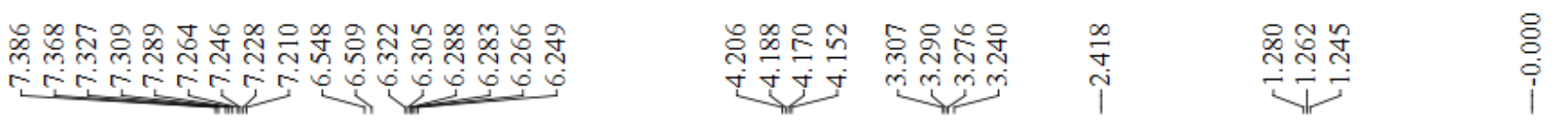

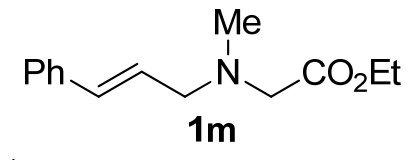

${ }^{1} \mathrm{H}$ NMR $\left(400 \mathrm{MHz}, \mathrm{CDCl}_{3}\right)$

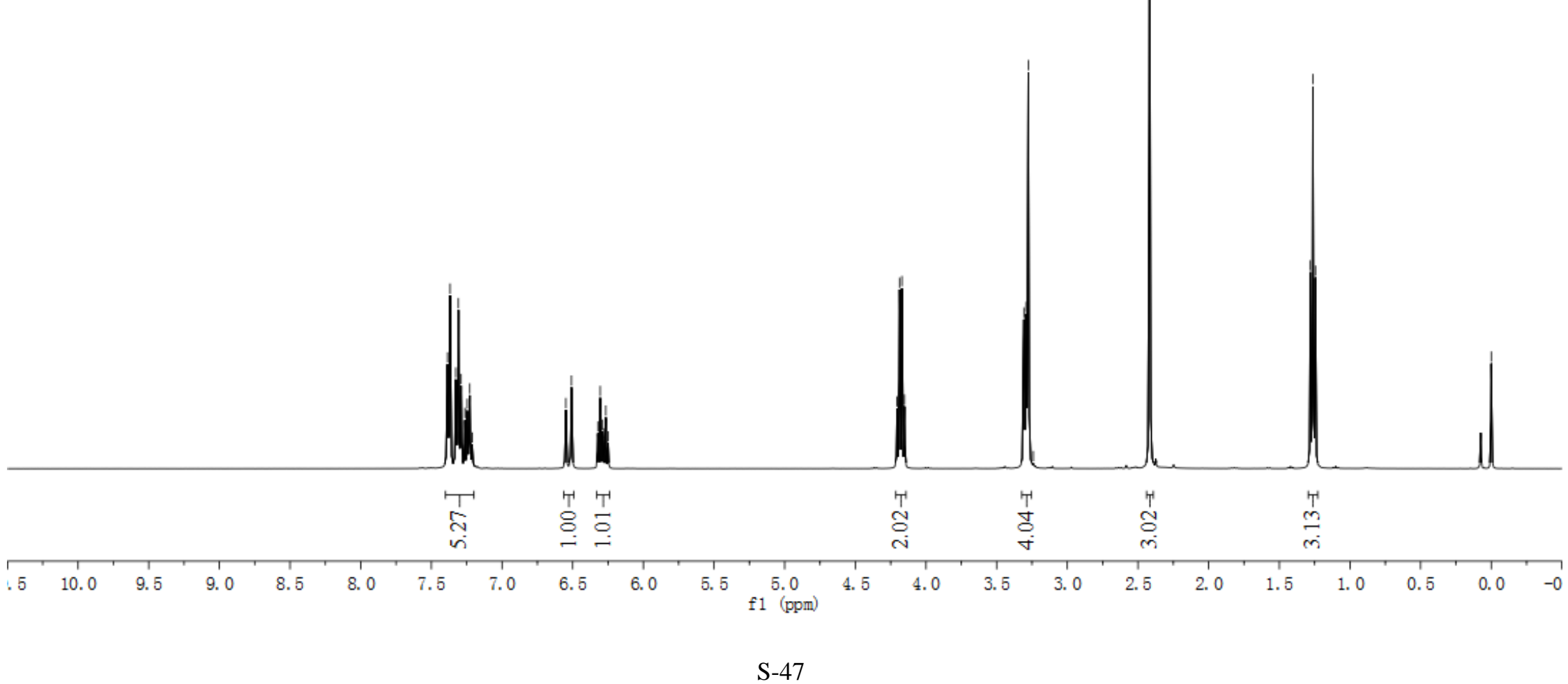




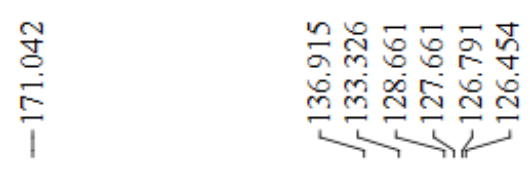

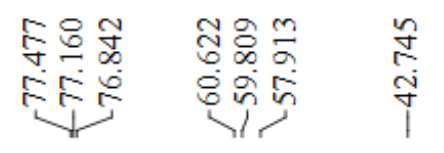

के

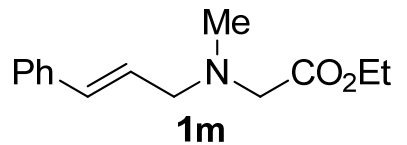

$\left.{ }^{13} \mathrm{C} \mathrm{NMR} \mathrm{(100} \mathrm{MHz,} \mathrm{CDCl}_{3}\right)$

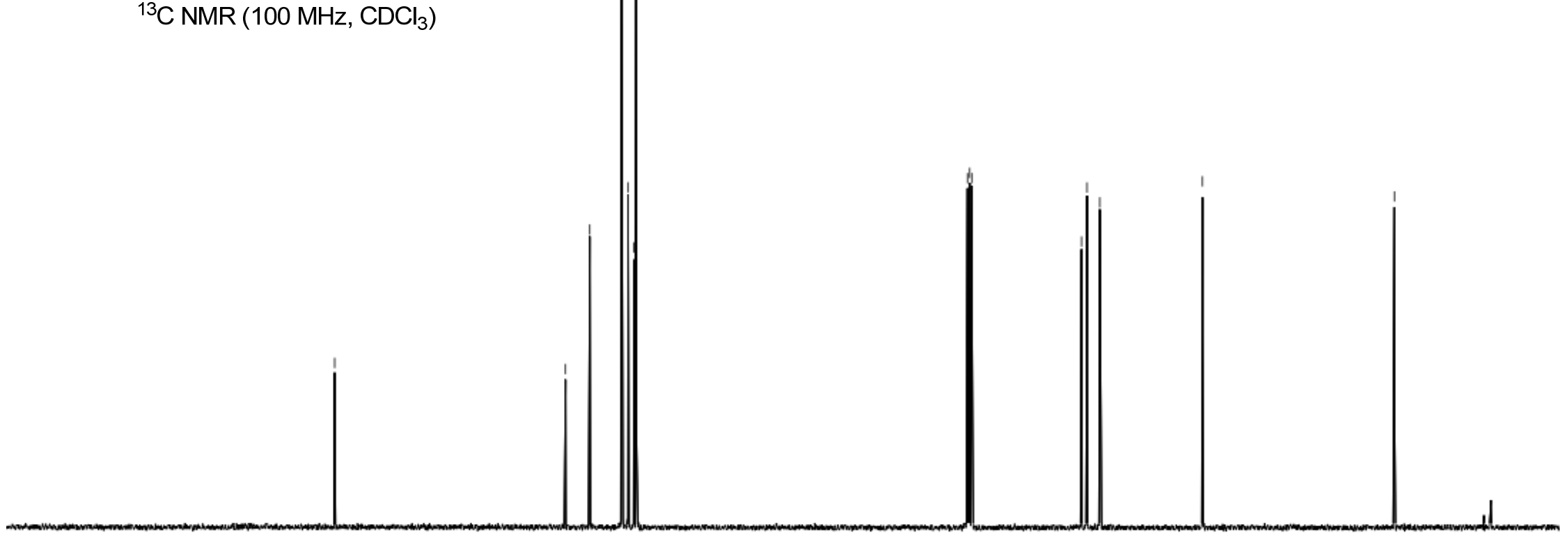

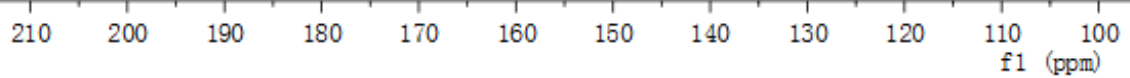




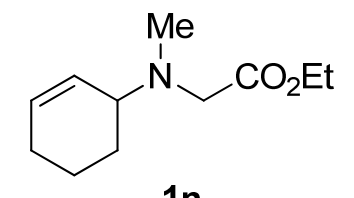

${ }^{1} \mathrm{HNMR}\left(400 \mathrm{MHz}, \mathrm{CDCl}_{3}\right)$

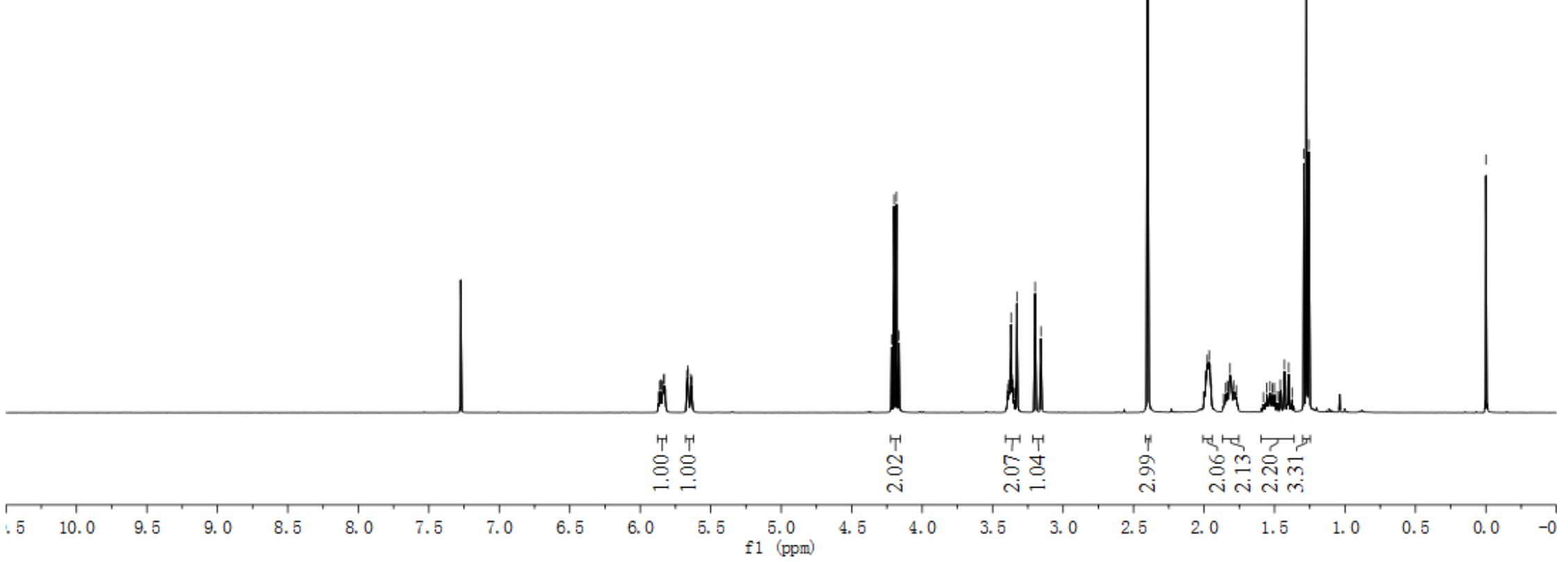




\begin{tabular}{|c|c|c|c|}
\hline$\frac{\stackrel{\circ}{\circ}}{\stackrel{\circ}{E}}$ & 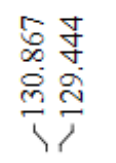 & 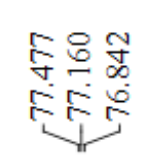 & 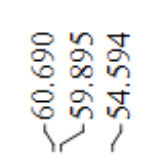 \\
\hline
\end{tabular}

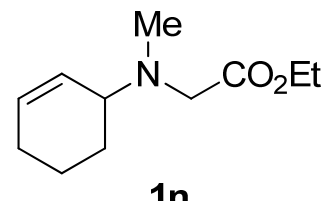

${ }^{13} \mathrm{C}$ NMR $\left(100 \mathrm{MHz}, \mathrm{CDCl}_{3}\right)$

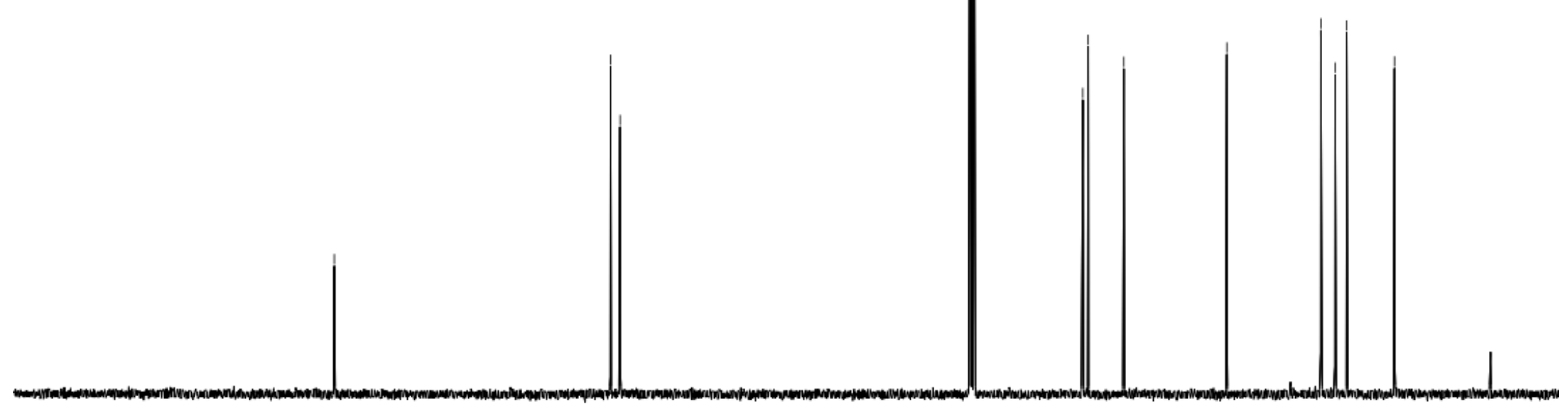

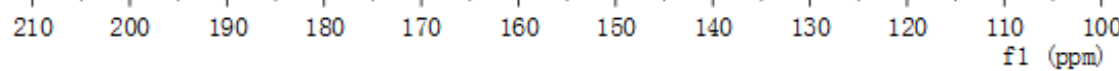




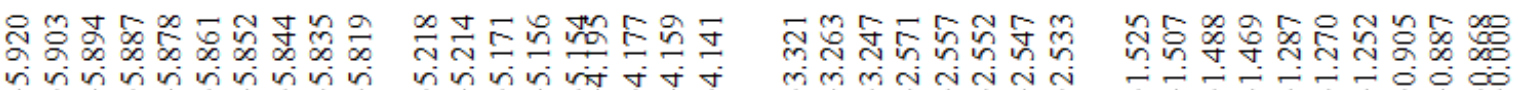

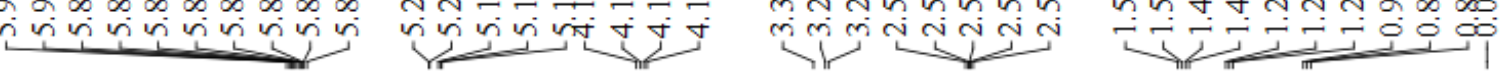

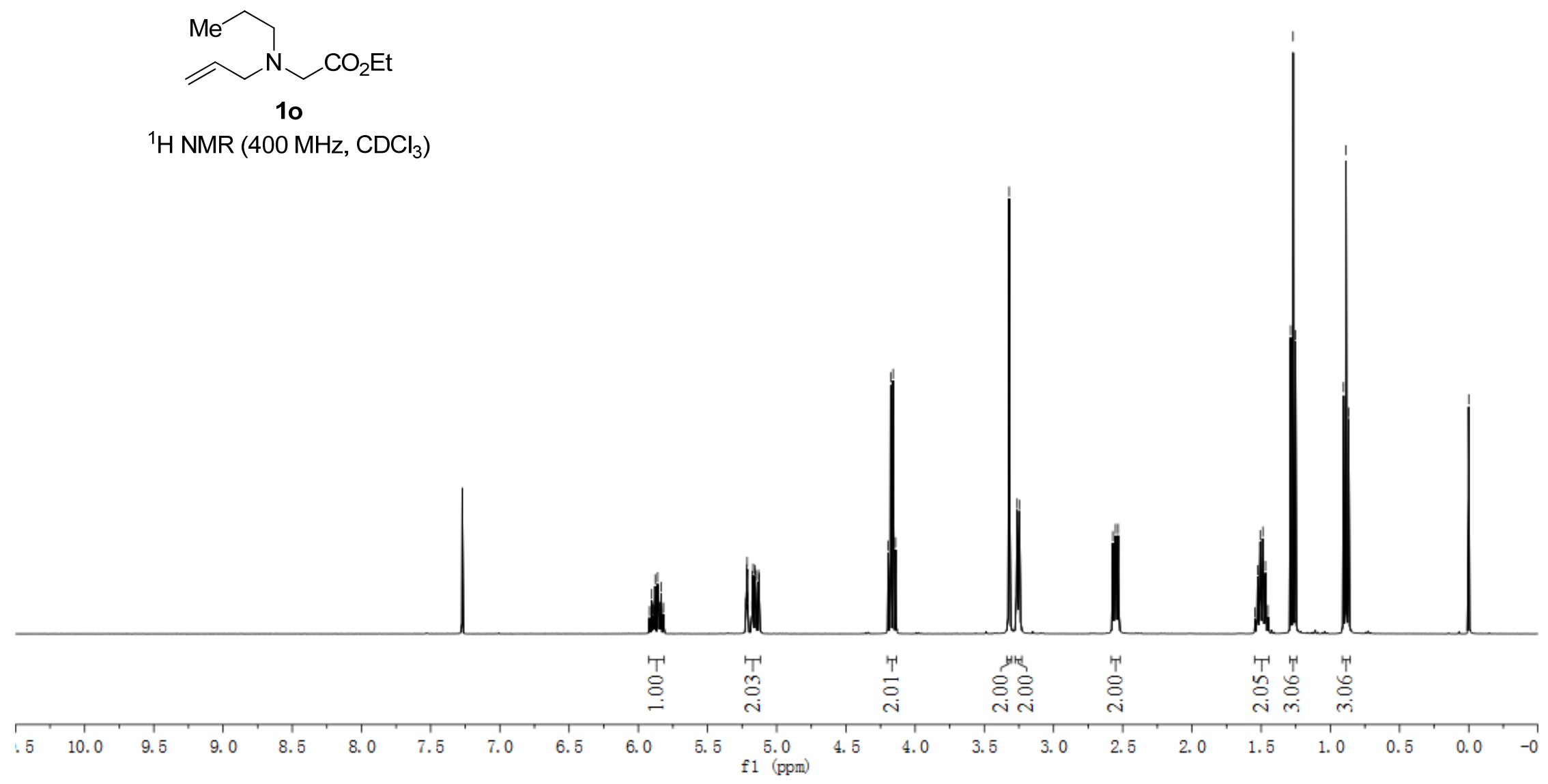



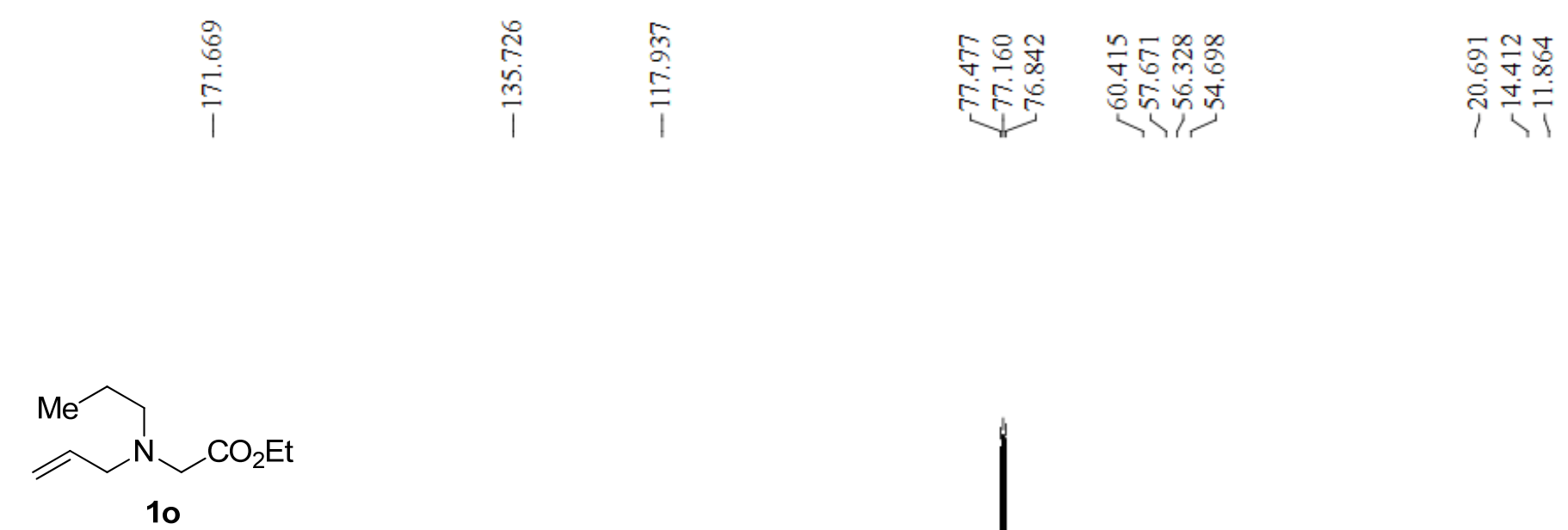

${ }^{13} \mathrm{C} \mathrm{NMR}\left(100 \mathrm{MHz}, \mathrm{CDCl}_{3}\right)$
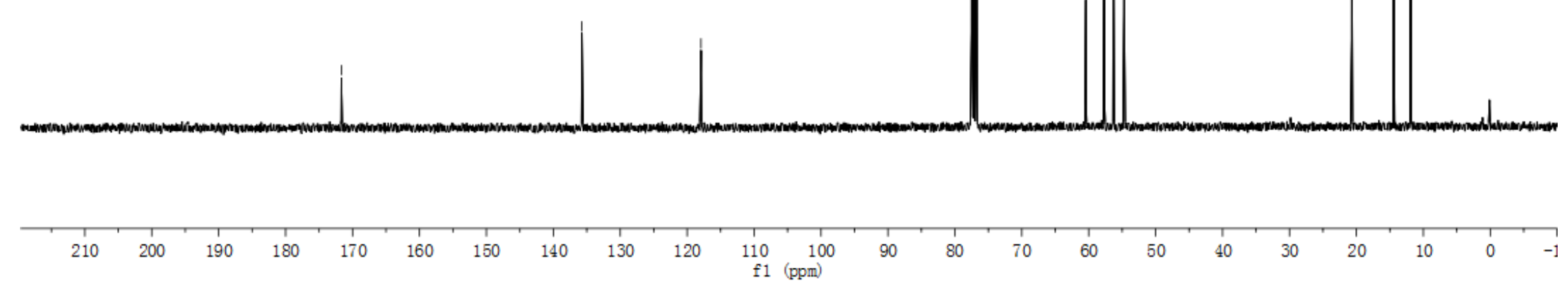


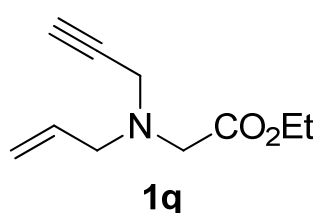

${ }^{1} \mathrm{H}$ NMR $\left(400 \mathrm{MHz}, \mathrm{CDCl}_{3}\right)$

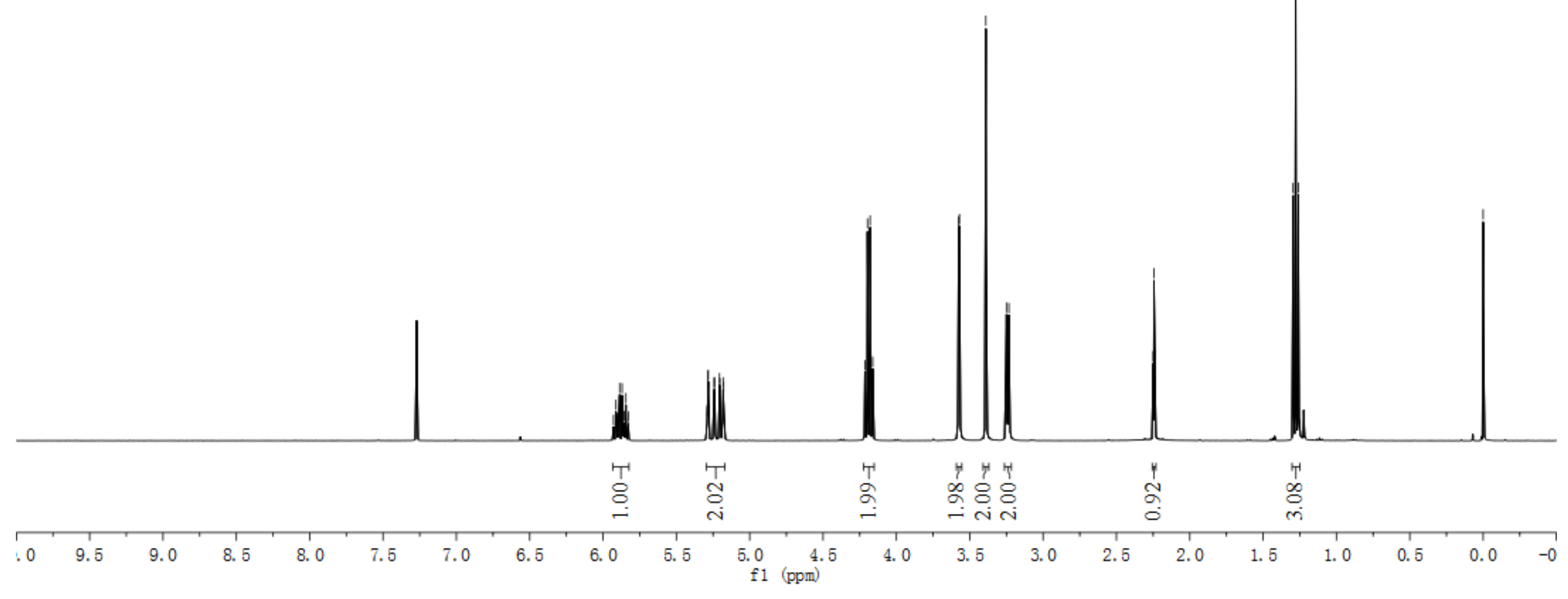




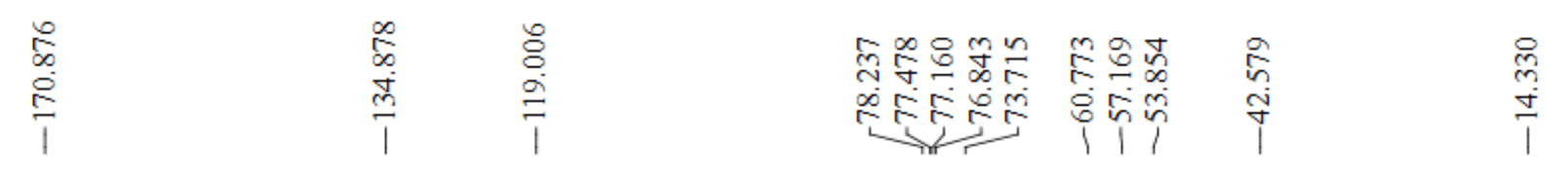

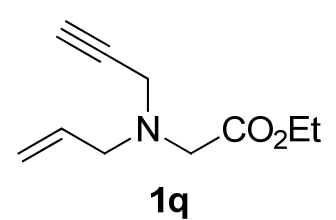

${ }^{13} \mathrm{C}$ NMR $\left(100 \mathrm{MHz}, \mathrm{CDCl}_{3}\right)$
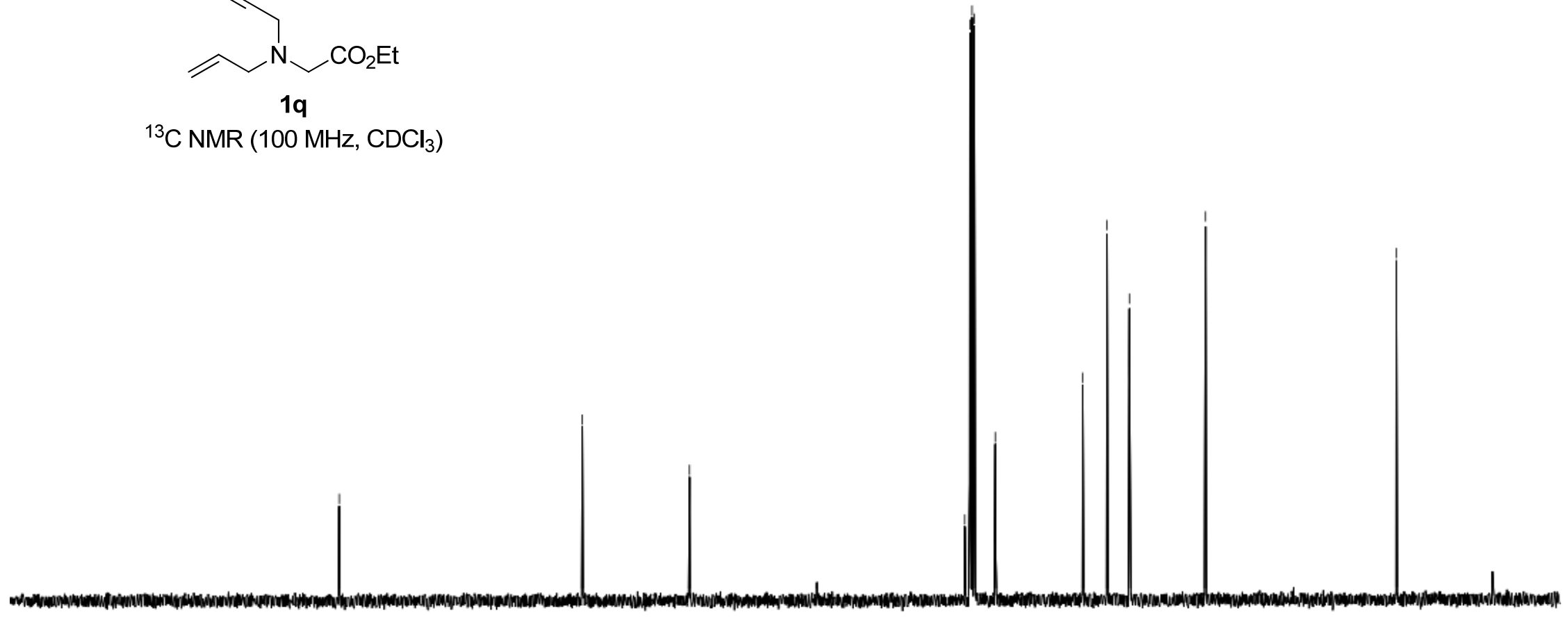

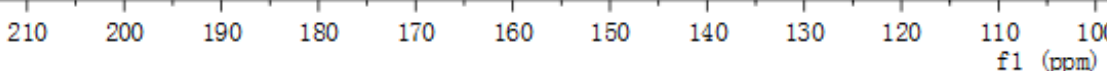




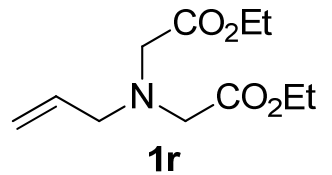

${ }^{1} \mathrm{H} \mathrm{NMR}\left(400 \mathrm{MHz}, \mathrm{CDCl}_{3}\right)$

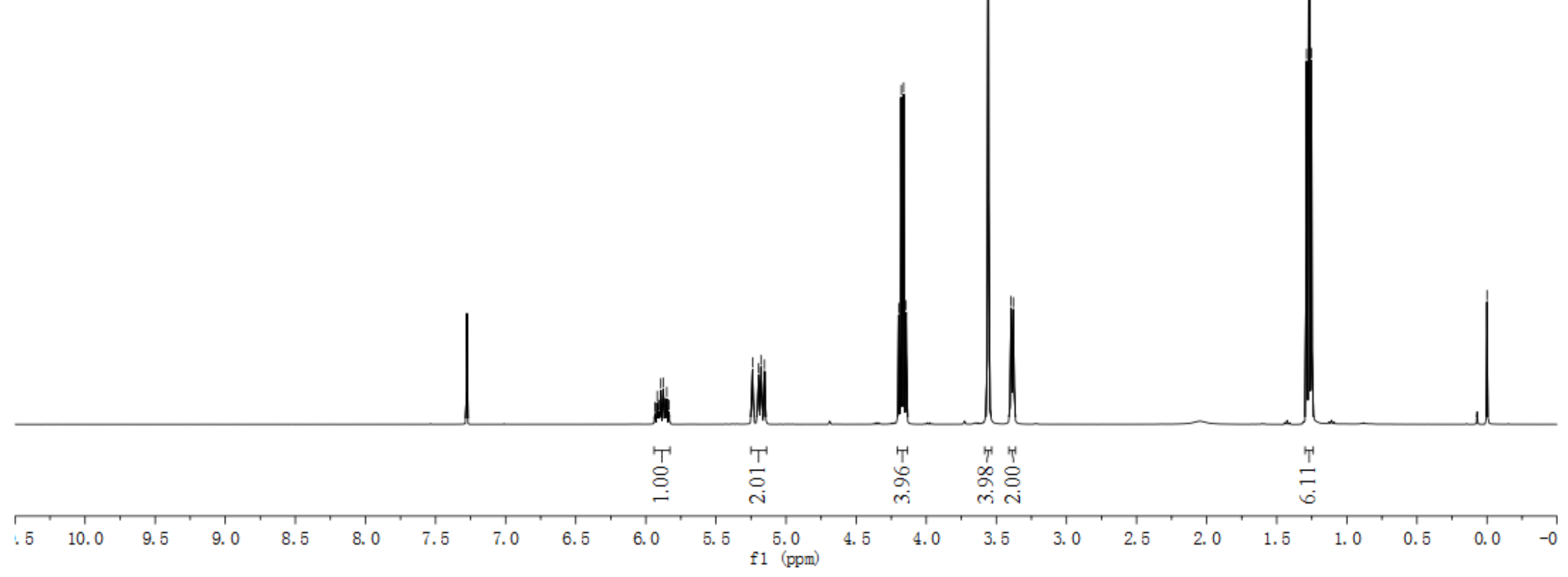




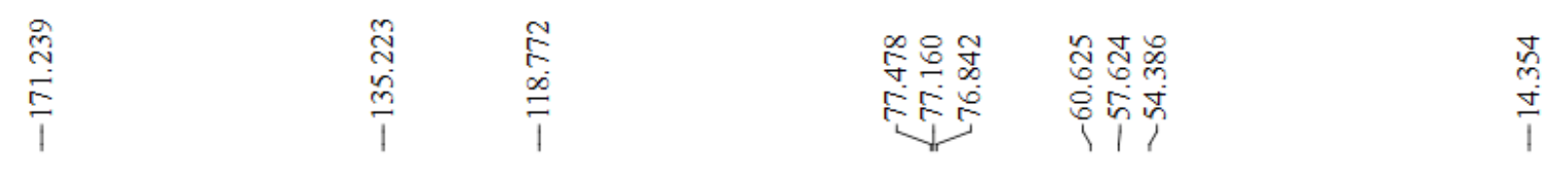

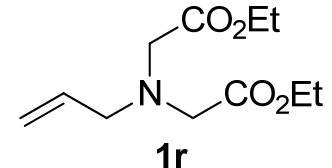

${ }^{13} \mathrm{C}$ NMR (100 MHz, $\left.\mathrm{CDCl}_{3}\right)$

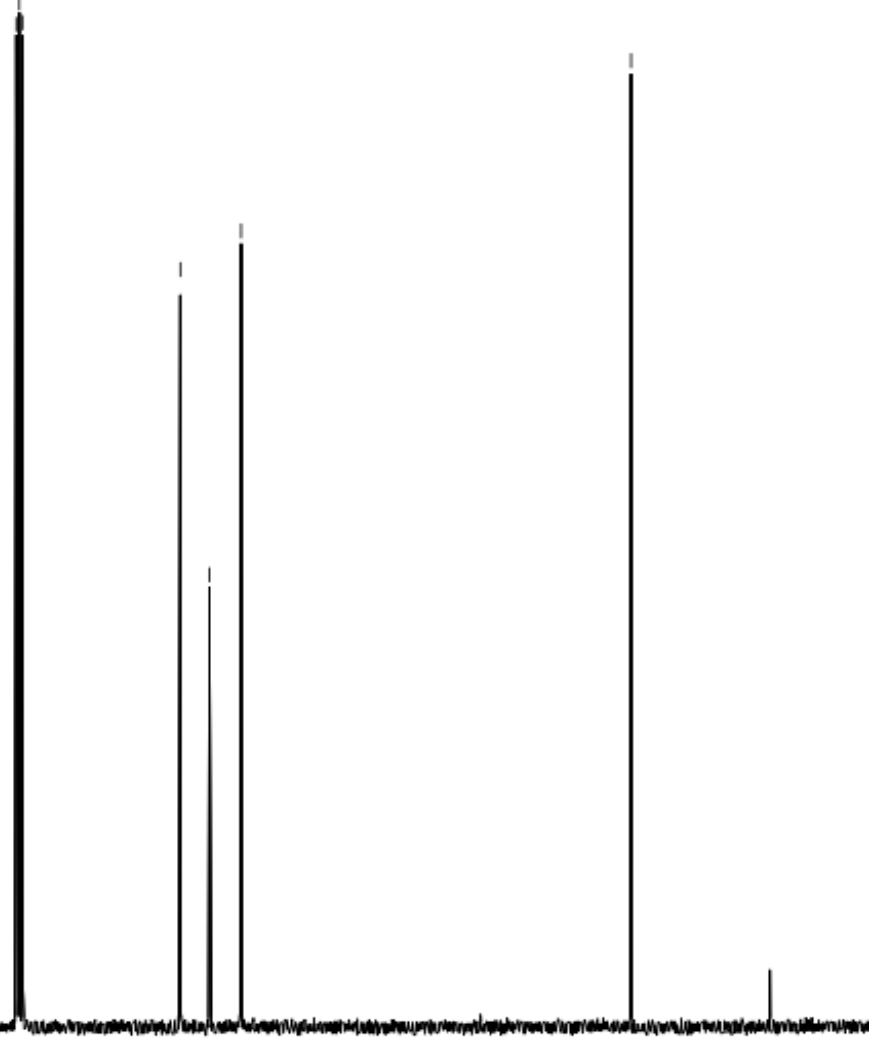

$\begin{array}{llllllllllll}210 & 200 & 190 & 180 & 170 & 160 & 150 & 140 & 130 & 120 & 110 & 100 \\ & & & & & & & & & & & \end{array}$ 


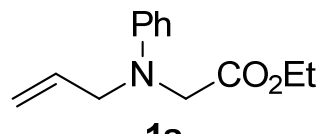

15

${ }^{1} \mathrm{H}$ NMR (400 MHz, $\mathrm{CDCl}_{3}$ )

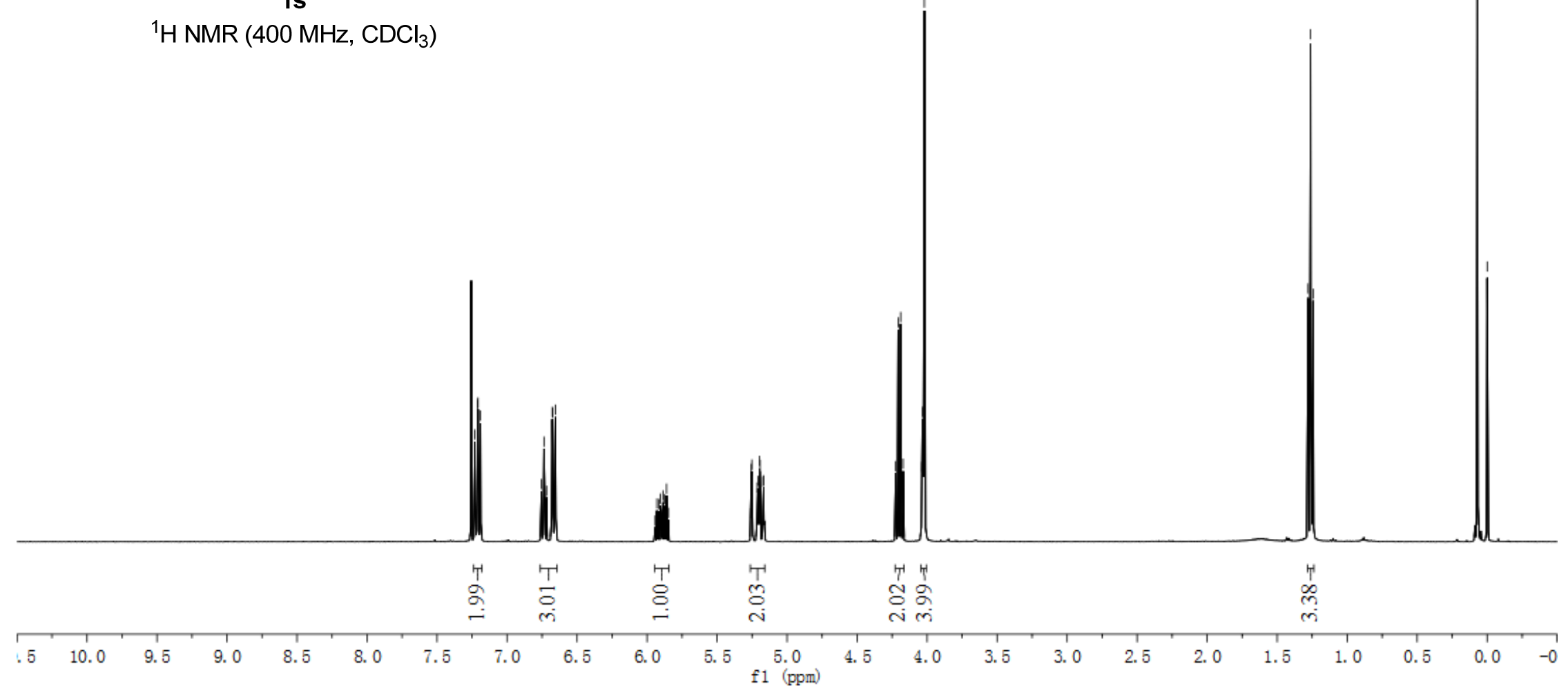




\begin{tabular}{|c|c|c|c|c|}
\hline$\underset{\mathbb{F}}{\stackrel{\widetilde{f}}{\Xi}}$ & 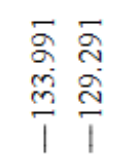 & 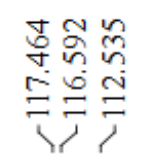 & 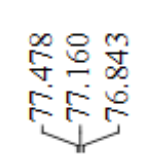 & 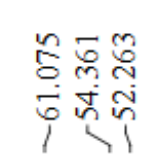 \\
\hline
\end{tabular}

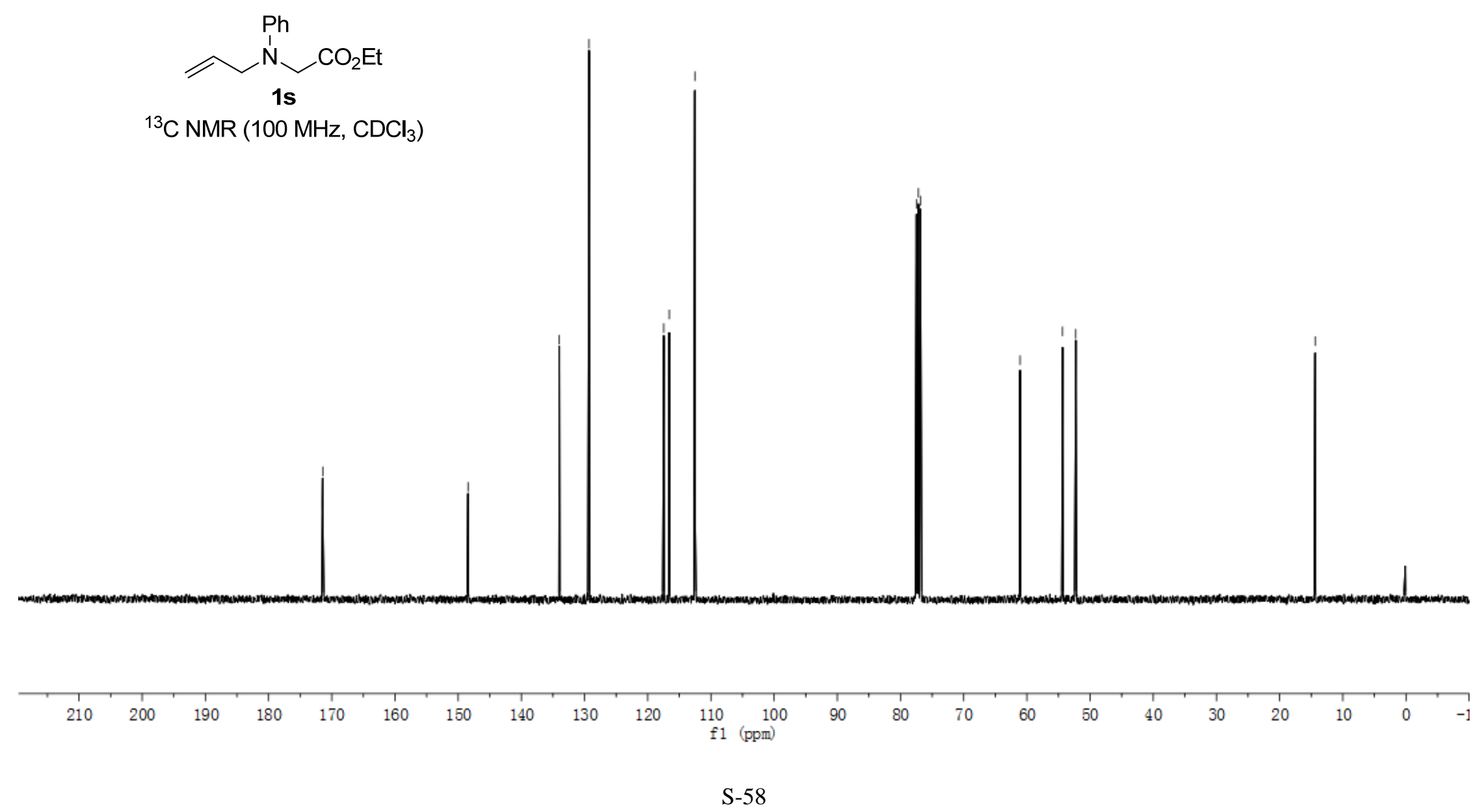




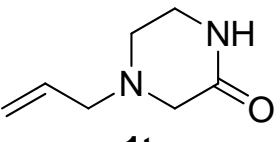

$1 t$

${ }^{1} \mathrm{H}$ NMR $\left(400 \mathrm{MHz}, \mathrm{CDCl}_{3}\right)$

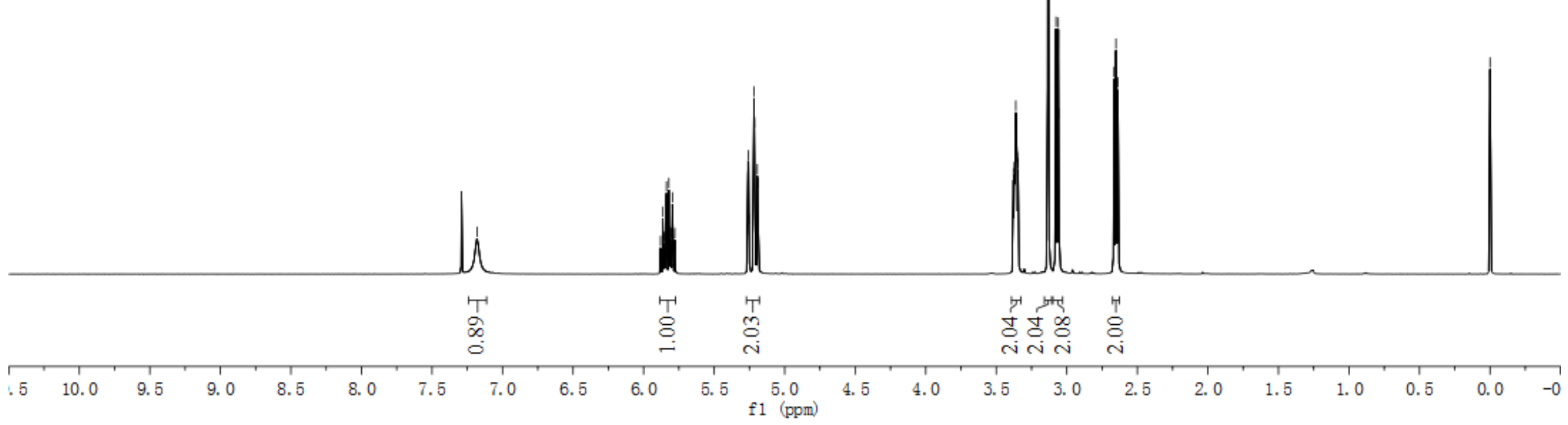




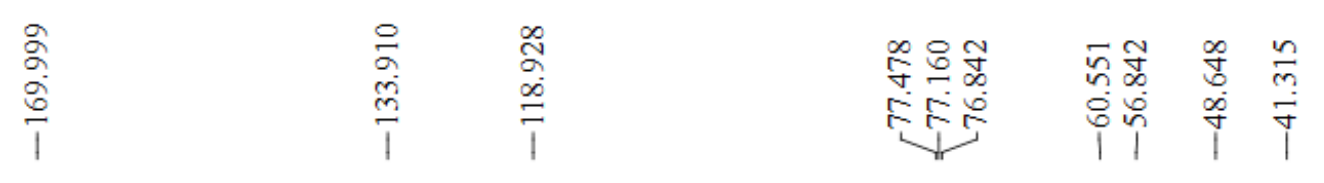

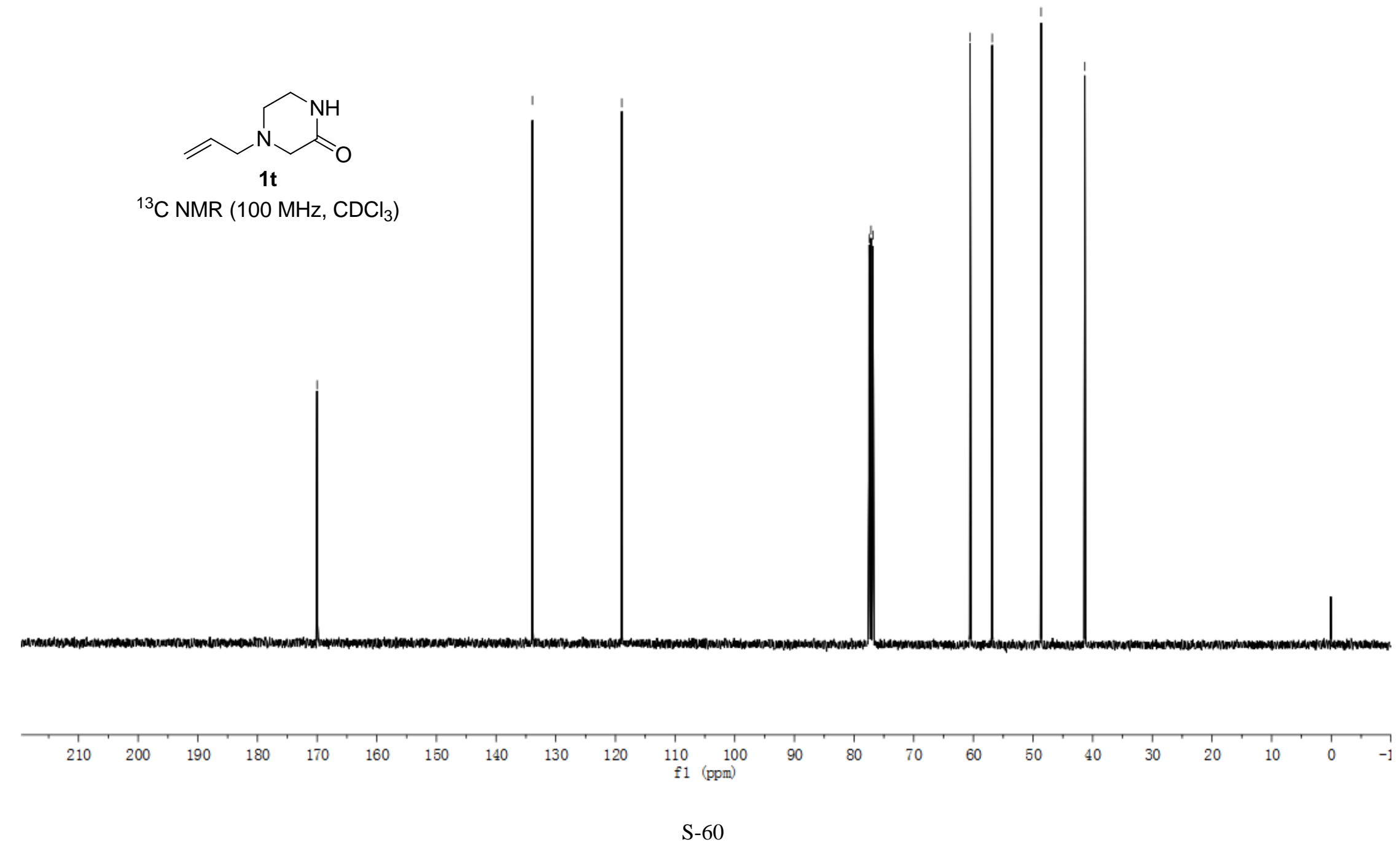




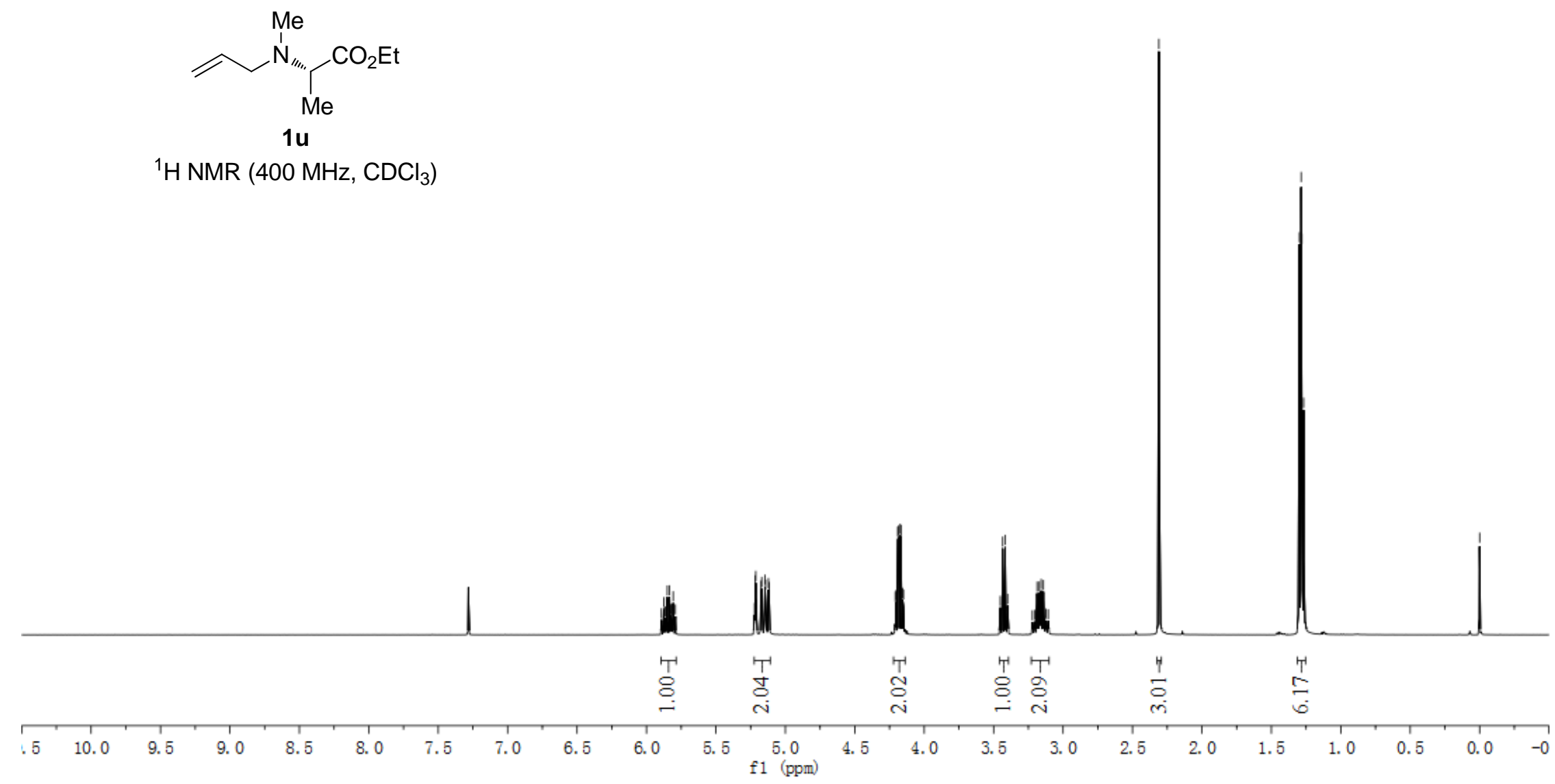




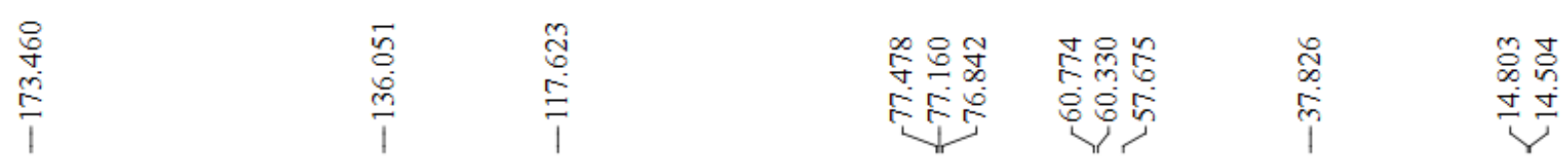

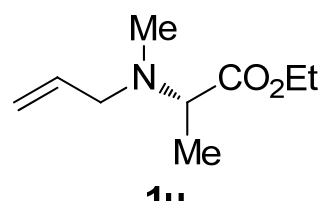

$1 \mathrm{u}$

${ }^{13} \mathrm{C}$ NMR (100 MHz, $\left.\mathrm{CDCl}_{3}\right)$

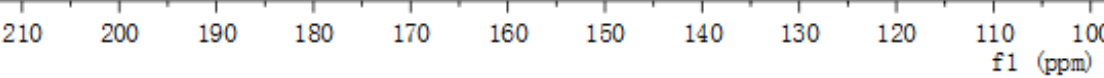




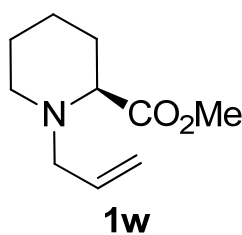

${ }^{1} \mathrm{H} \mathrm{NMR}\left(400 \mathrm{MHz}, \mathrm{CDCl}_{3}\right)$

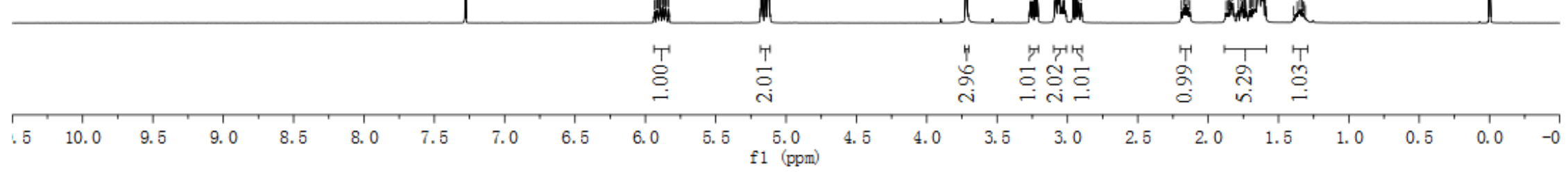



$\underset{\substack{\mathfrak{1} \\ \mathfrak{I}}}{\stackrel{1}{7}}$

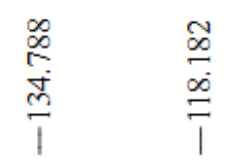

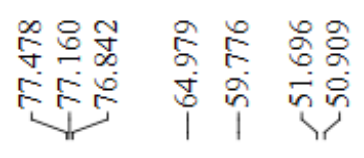
$\infty \bar{D} \bar{\infty}$
จ่ำ

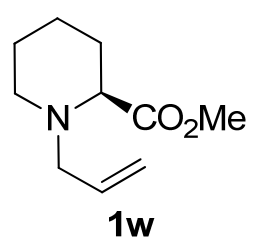

${ }^{13} \mathrm{C} \mathrm{NMR}\left(100 \mathrm{MHz}, \mathrm{CDCl}_{3}\right)$

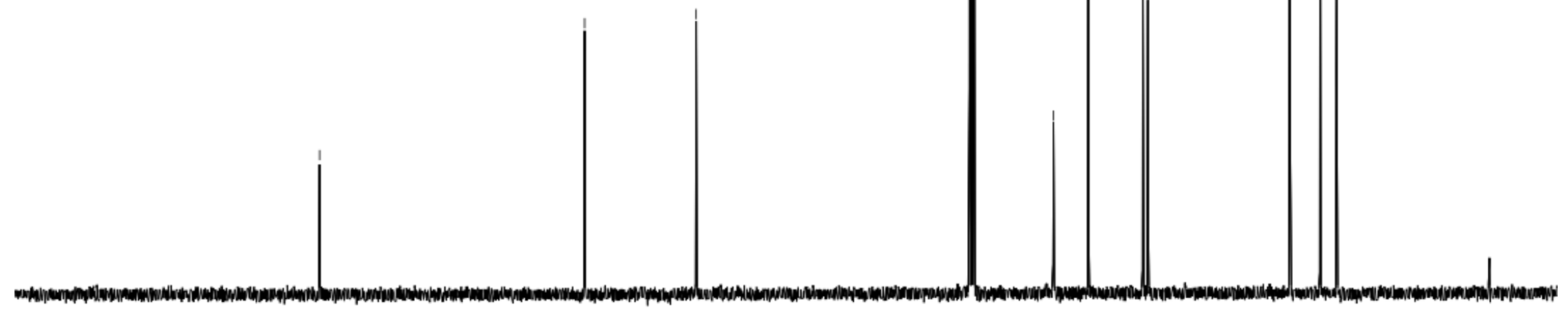

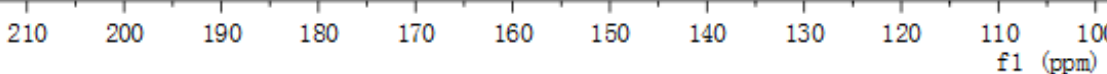




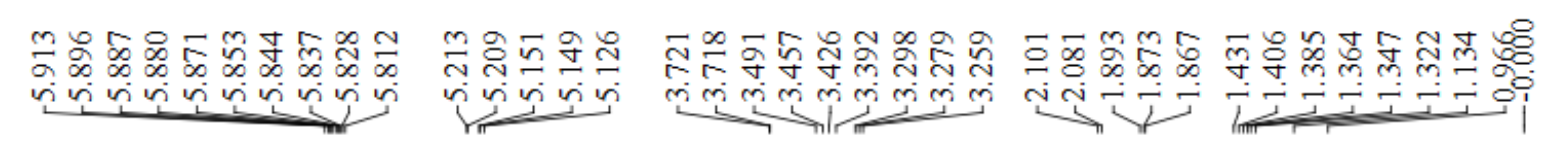

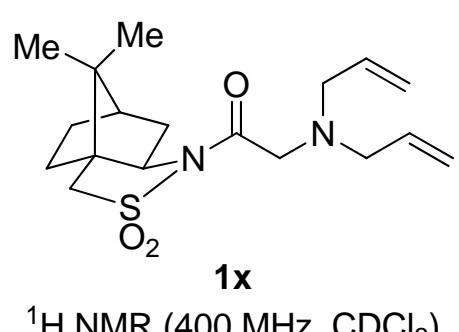

${ }^{1} \mathrm{H}$ NMR $\left(400 \mathrm{MHz}, \mathrm{CDCl}_{3}\right)$

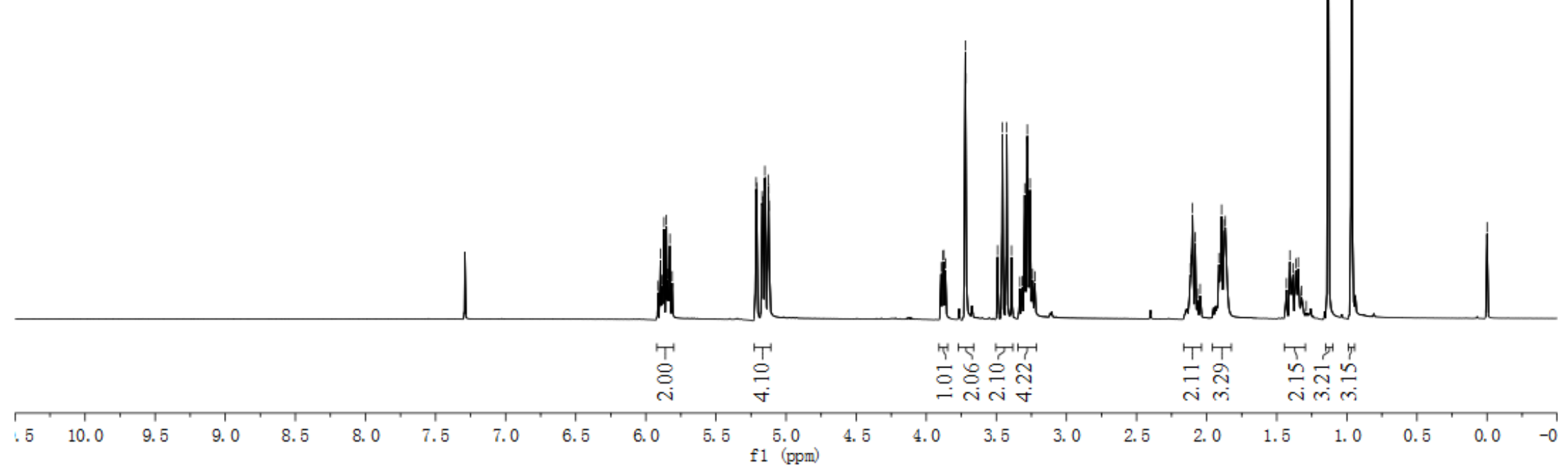



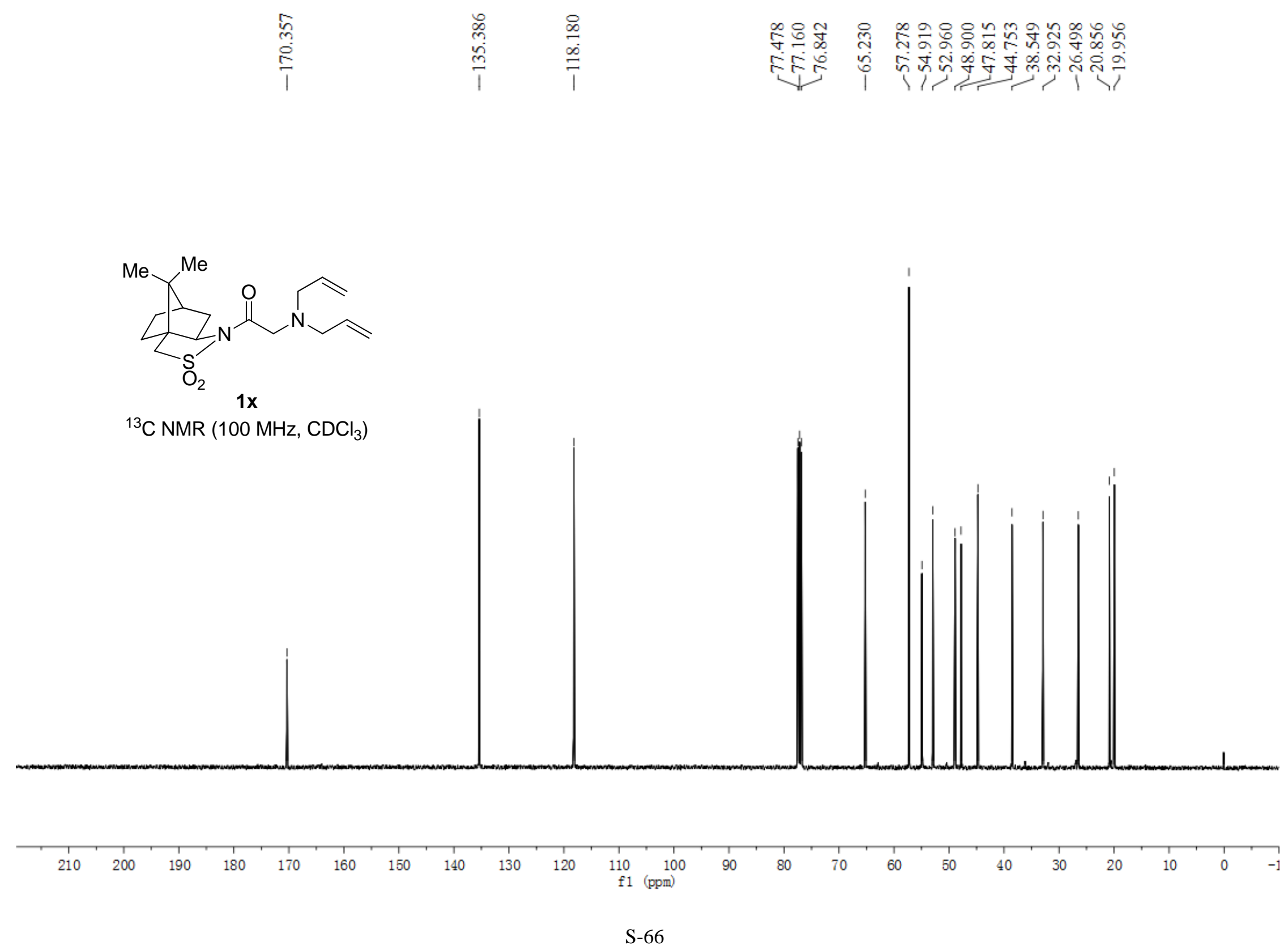


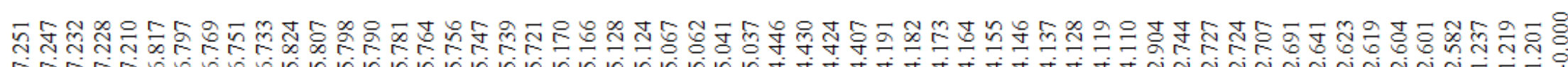

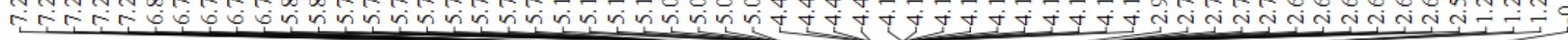

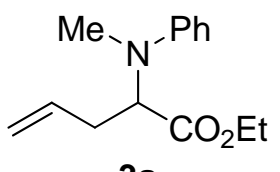

$3 a$

${ }^{1} \mathrm{H} \mathrm{NMR}\left(400 \mathrm{MHz}, \mathrm{CDCl}_{3}\right)$

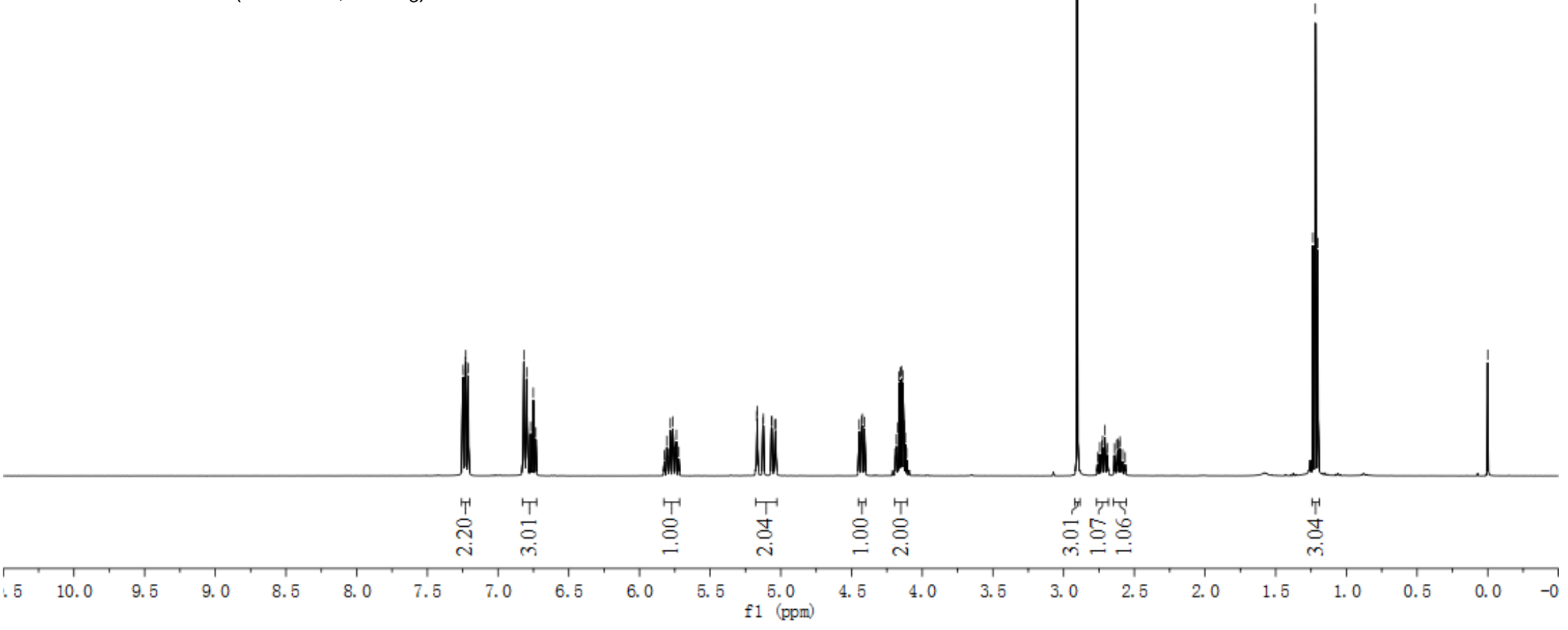




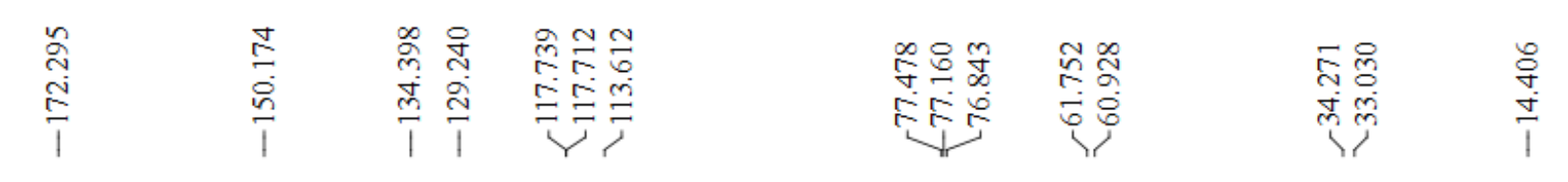

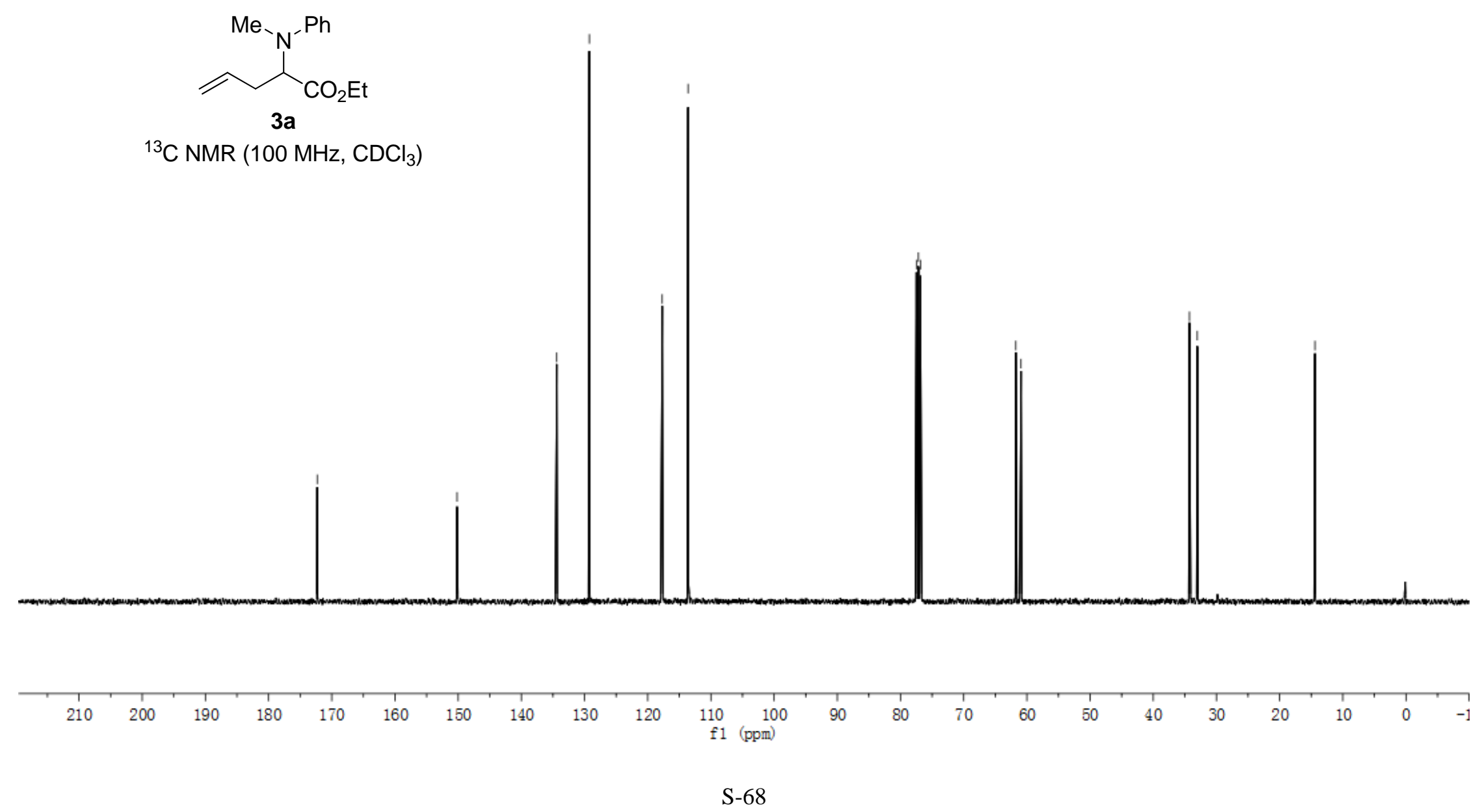




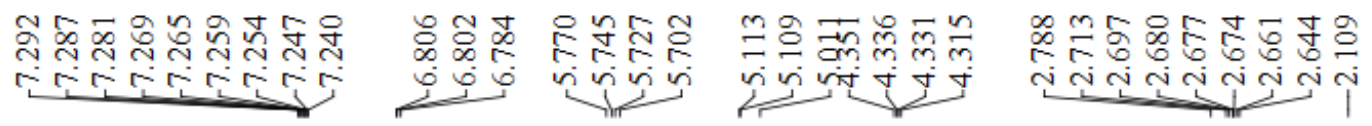

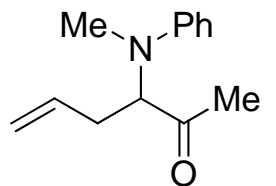

3b

${ }^{1} \mathrm{H}$ NMR $\left(400 \mathrm{MHz}, \mathrm{CDCl}_{3}\right)$

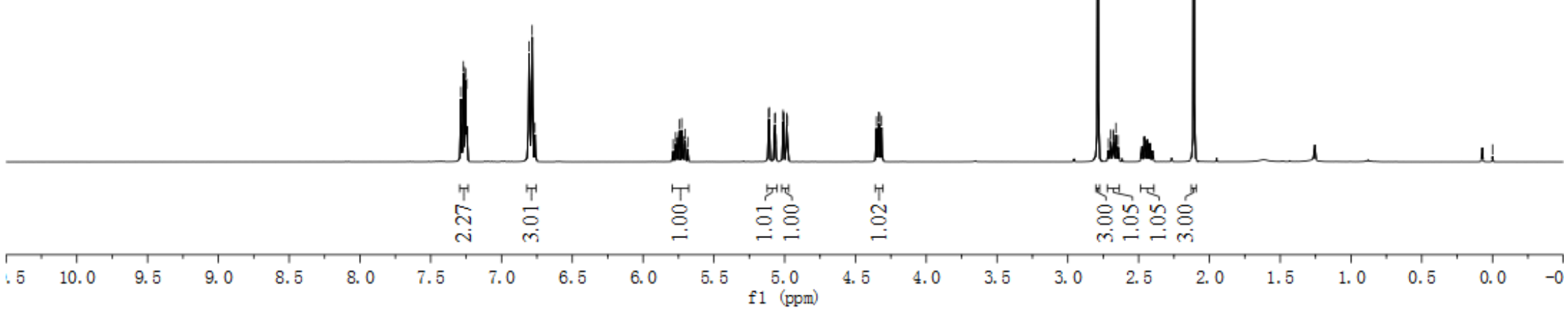




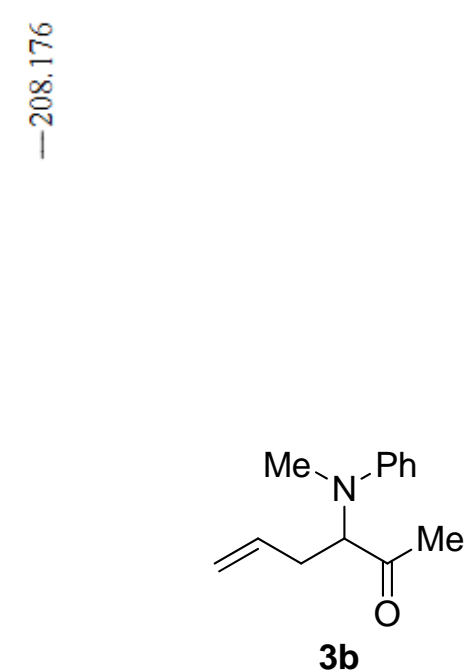

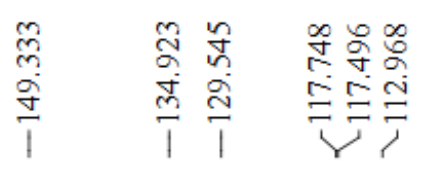

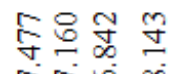

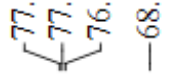

๙⿻心口

लेक

${ }^{3} \mathrm{C}$ NMR $\left(100 \mathrm{MHz}, \mathrm{CDCl}_{3}\right)$

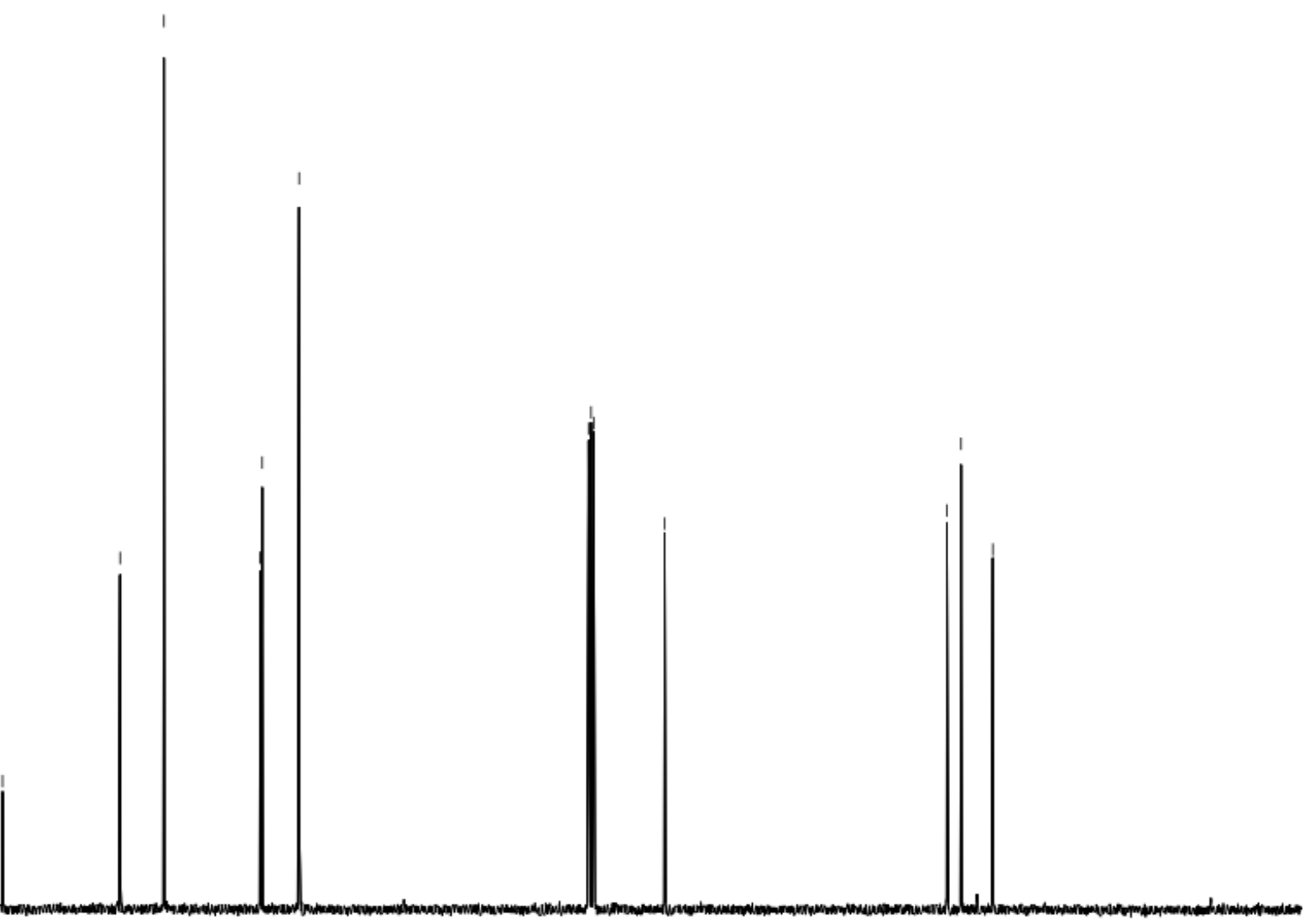

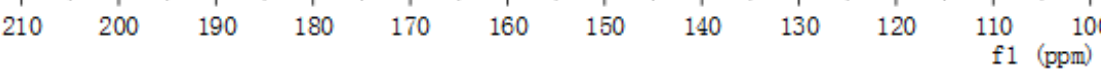




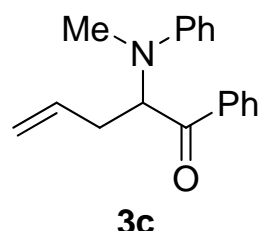

${ }^{1} \mathrm{H}$ NMR $\left(400 \mathrm{MHz}, \mathrm{CDCl}_{3}\right)$

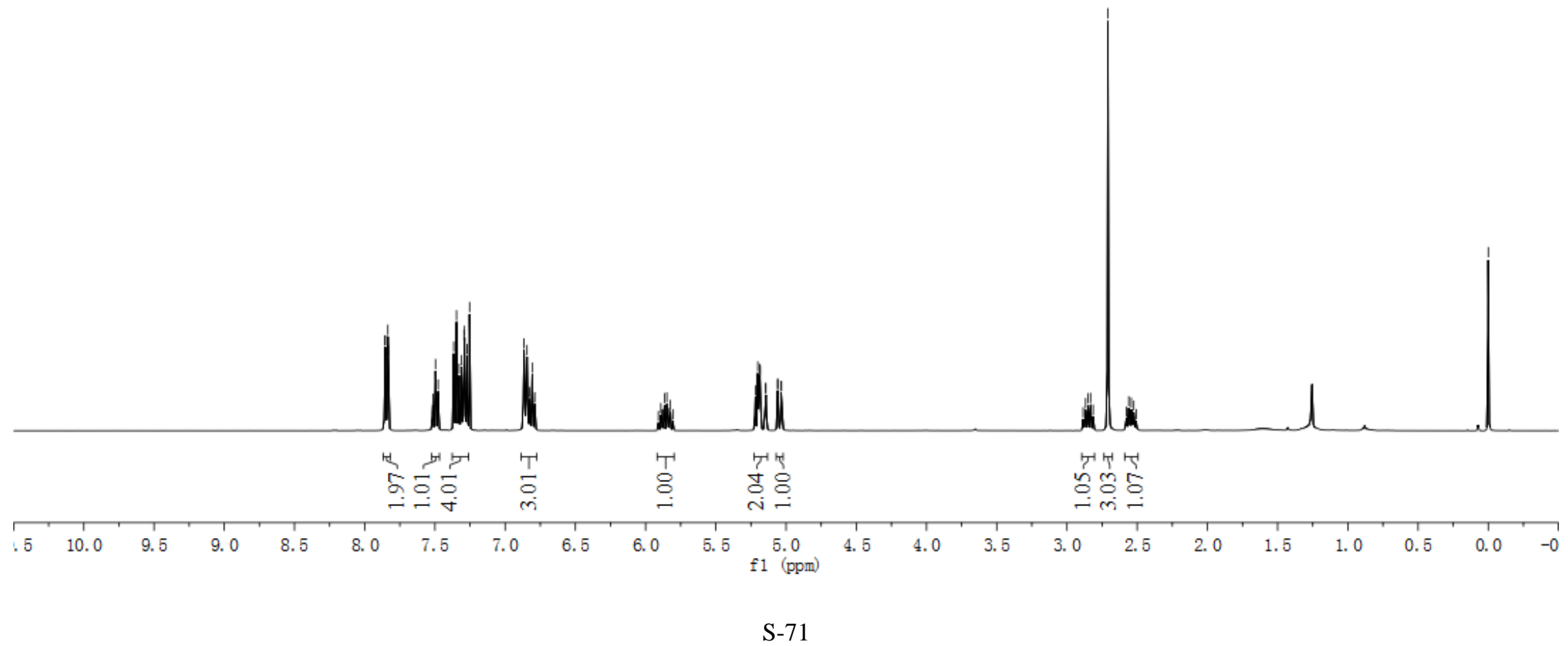




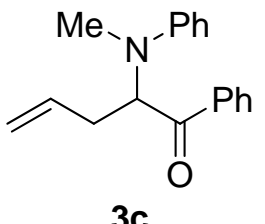

${ }^{13} \mathrm{C}$ NMR $\left(100 \mathrm{MHz}, \mathrm{CDCl}_{3}\right)$
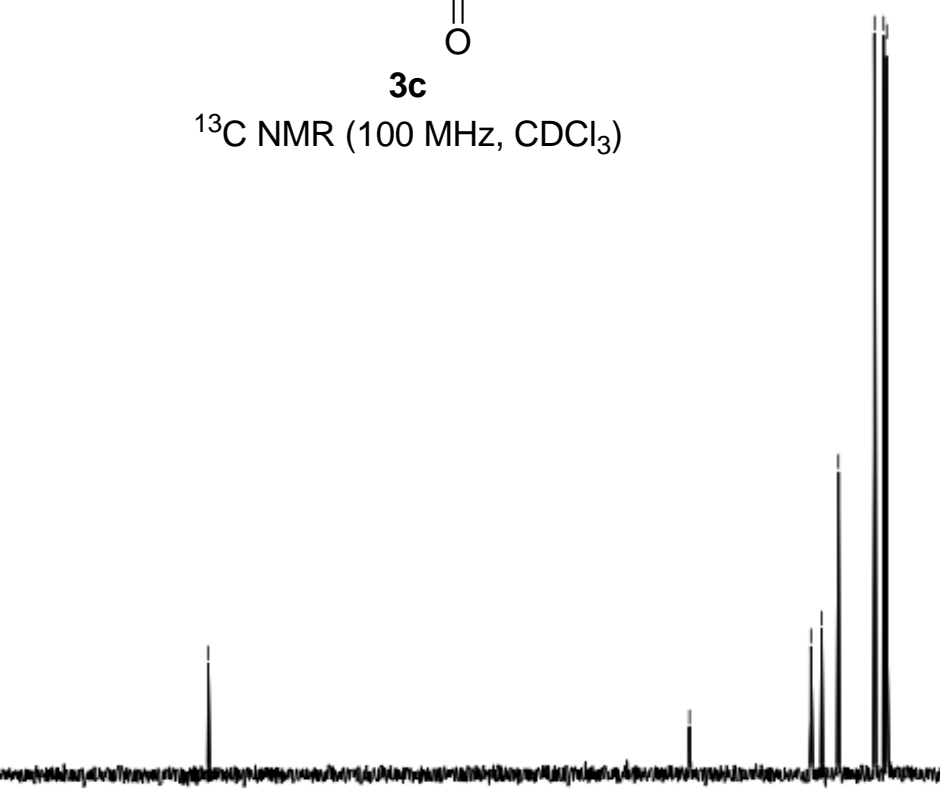

$210 \quad 200$

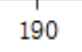

$180 \quad 170$

$160 \quad 150$

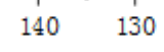

120

$\begin{array}{cc}110 & 100 \\ \mathrm{f} 1 & (\mathrm{ppm})\end{array}$
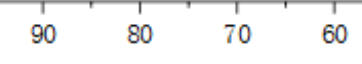

50
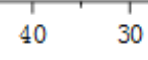


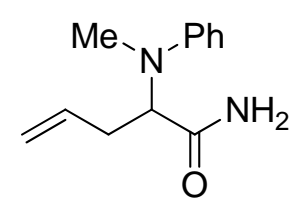

3d

${ }^{1} \mathrm{H}$ NMR $\left(400 \mathrm{MHz}, \mathrm{CDCl}_{3}\right)$

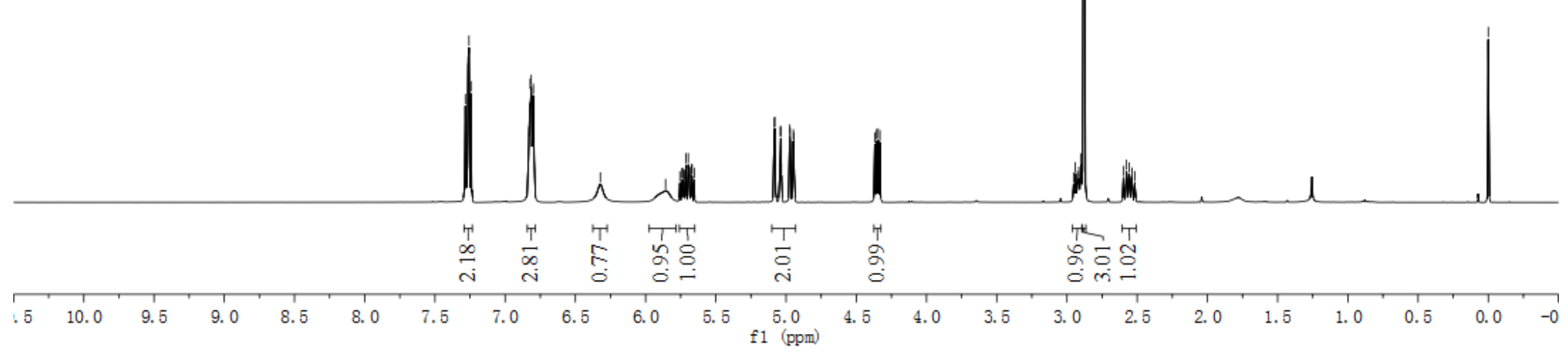




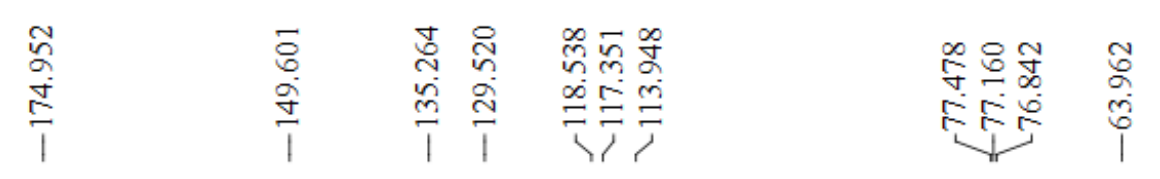

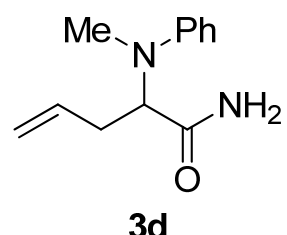

${ }^{13} \mathrm{C} \mathrm{NMR}\left(100 \mathrm{MHz}, \mathrm{CDCl}_{3}\right)$

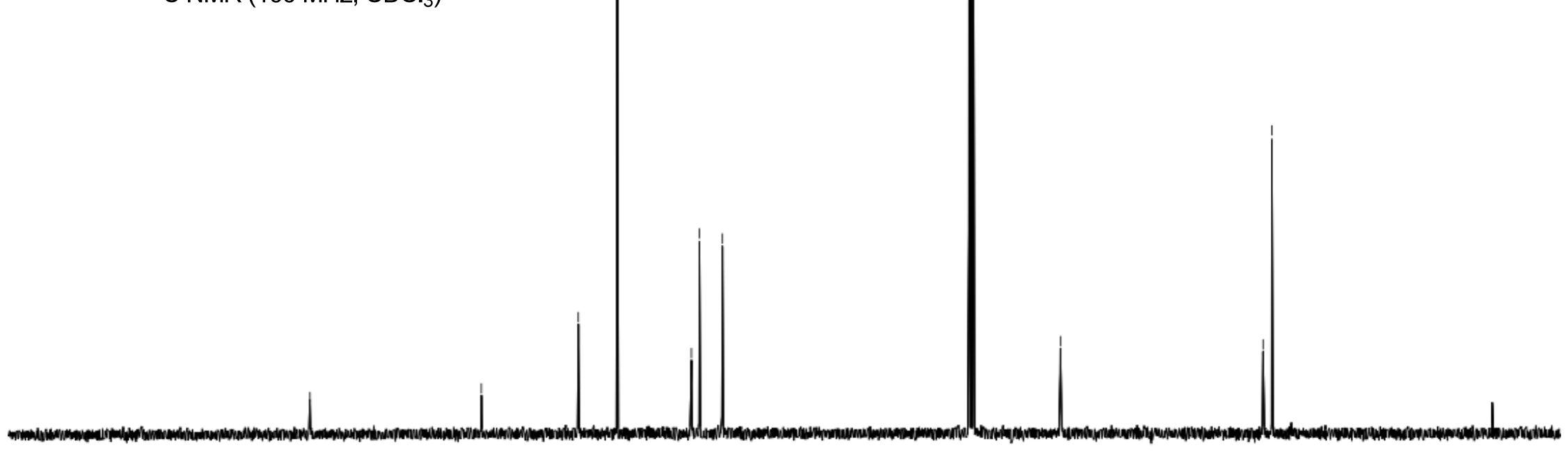

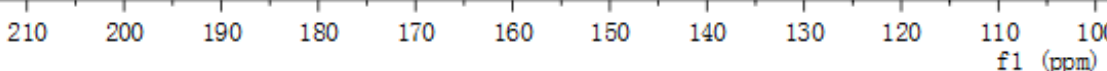




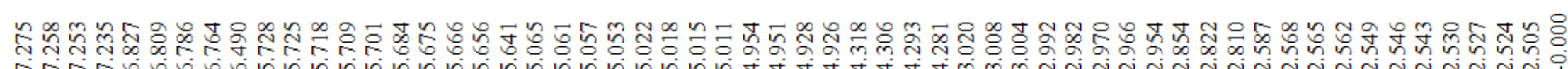

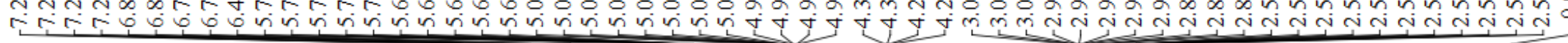

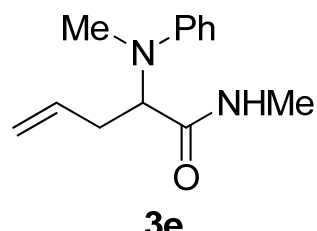

${ }^{1} \mathrm{H} \mathrm{NMR}\left(400 \mathrm{MHz}, \mathrm{CDCl}_{3}\right)$

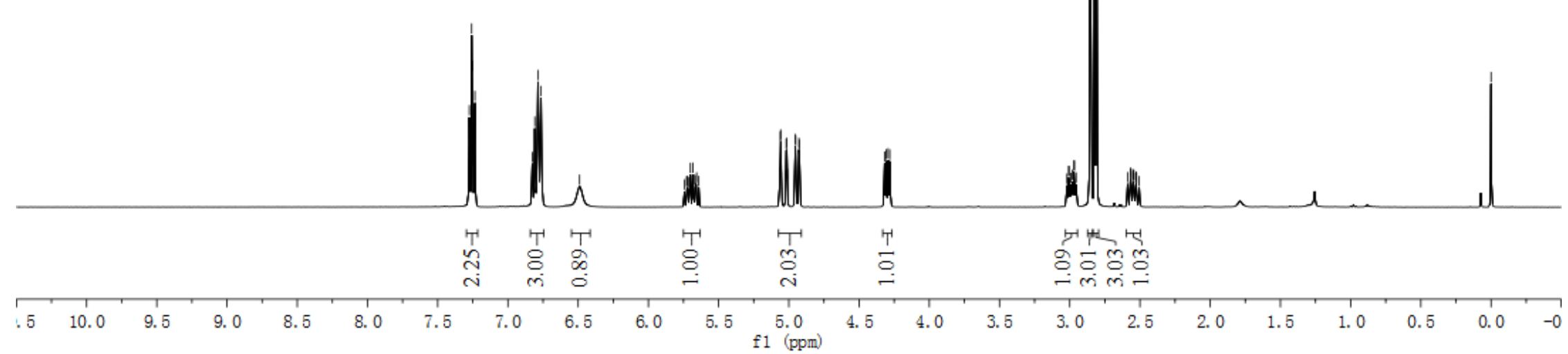




\begin{tabular}{|c|c|c|c|}
\hline $\begin{array}{l}\stackrel{2}{a} \\
\stackrel{\sigma}{\sigma} \\
\frac{\sigma}{1}\end{array}$ & $\begin{array}{ll}n & 8 \\
0 & 0 \\
n & 0 \\
m & 0 \\
1 & 1\end{array}$ & 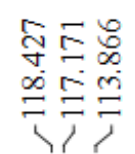 & 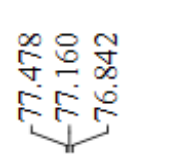 \\
\hline
\end{tabular}

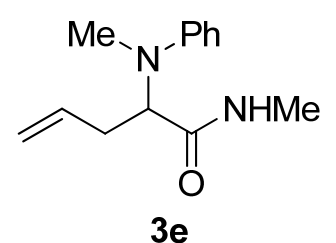

${ }^{13} \mathrm{C}$ NMR (100 MHz, $\left.\mathrm{CDCl}_{3}\right)$

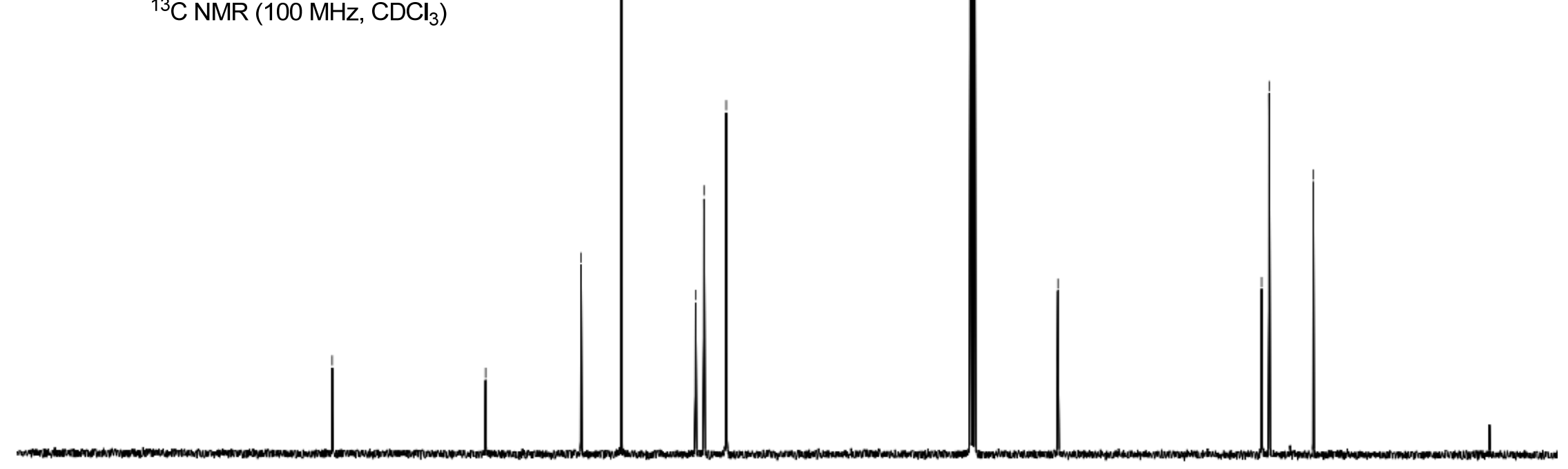

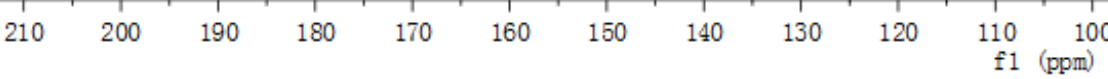




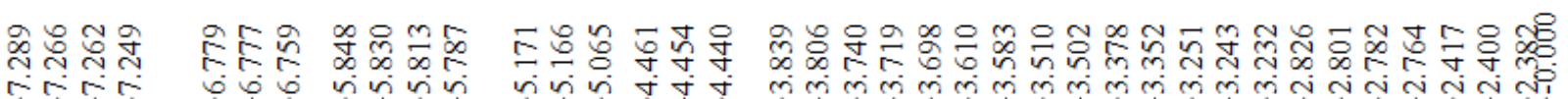

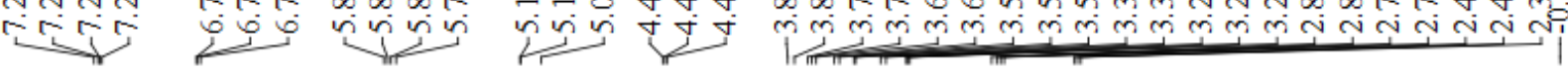

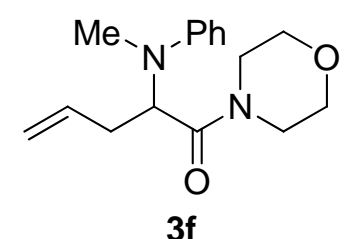

${ }^{1} \mathrm{H}$ NMR $\left(400 \mathrm{MHz}, \mathrm{CDCl}_{3}\right)$

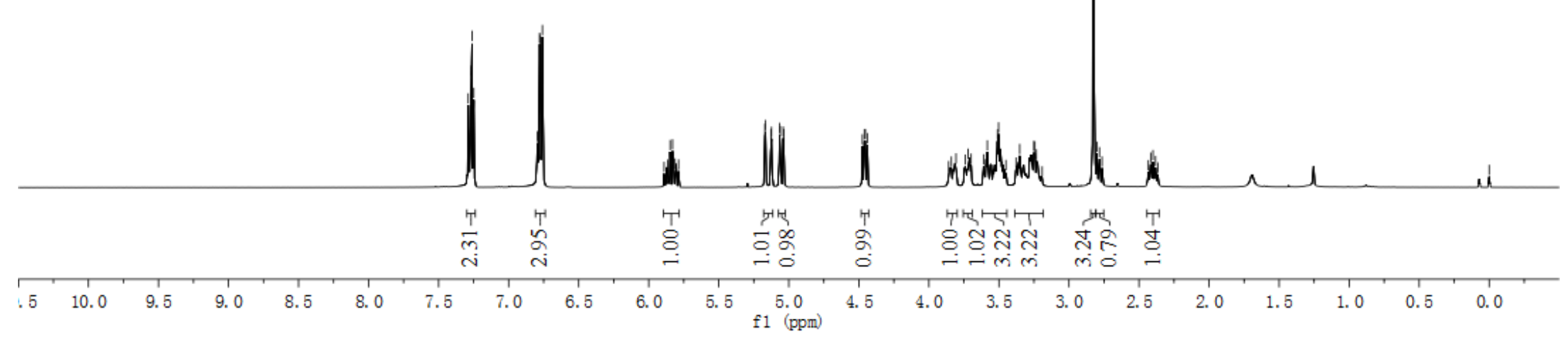




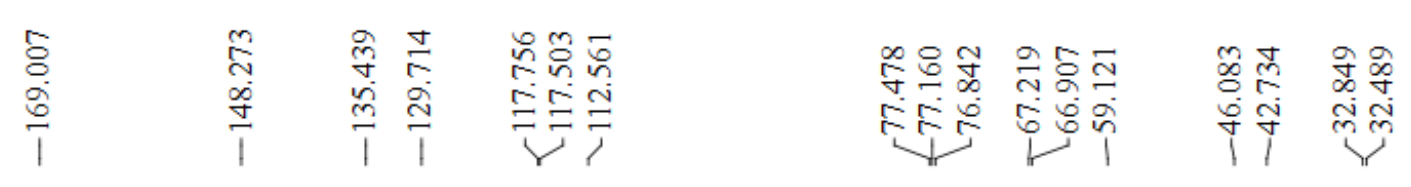

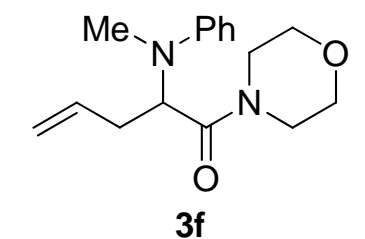

${ }^{13} \mathrm{C}$ NMR $\left(100 \mathrm{MHz}, \mathrm{CDCl}_{3}\right)$

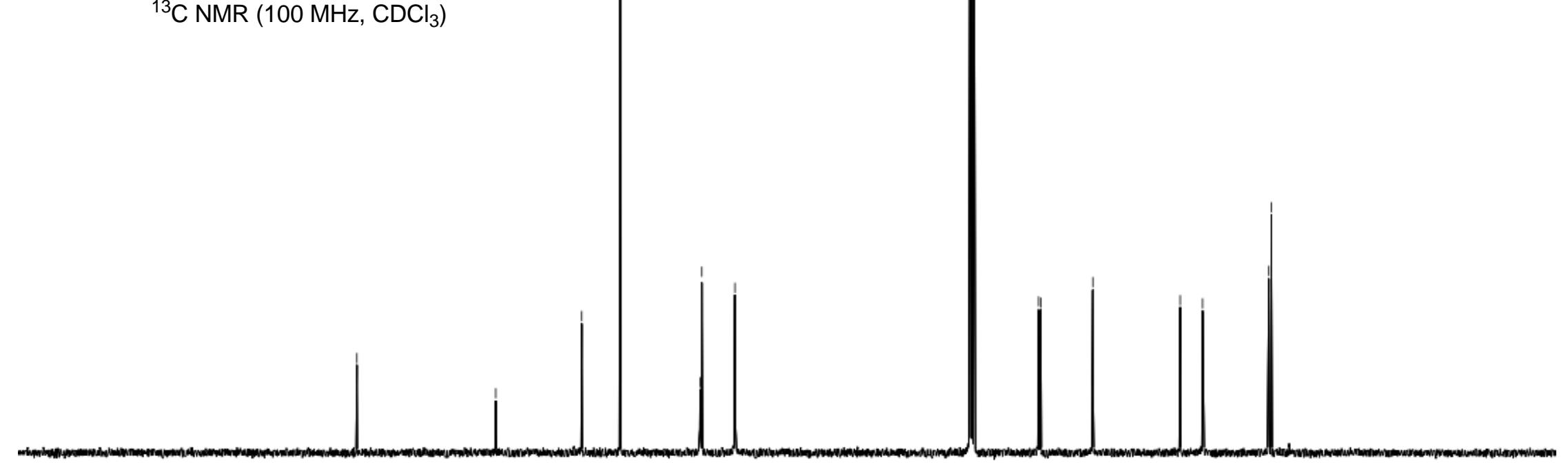

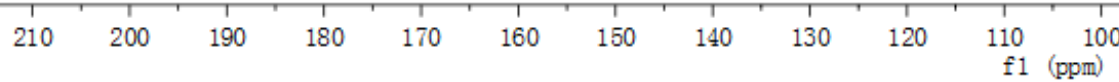




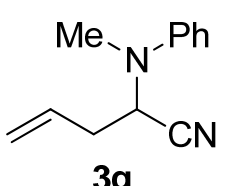

${ }^{1} \mathrm{H}$ NMR (400 MHz, $\mathrm{CDCl}_{3}$ )

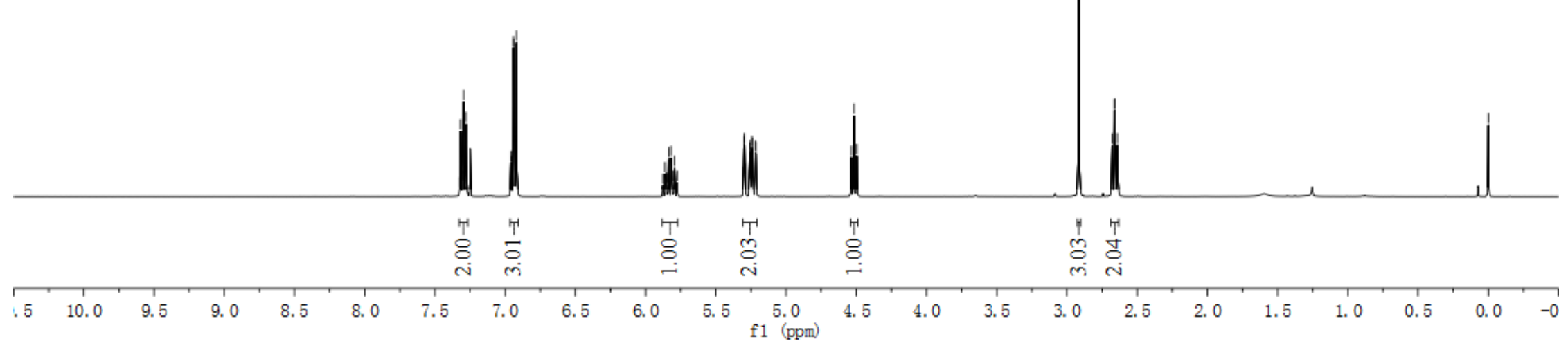




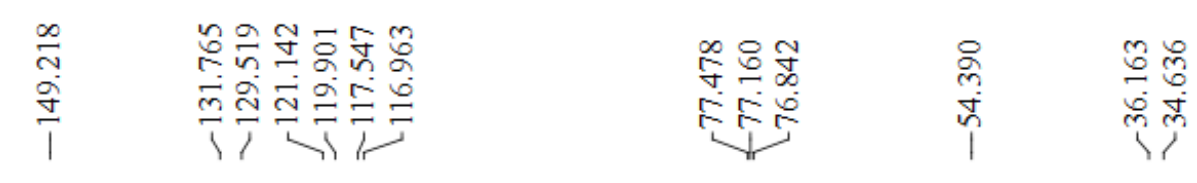

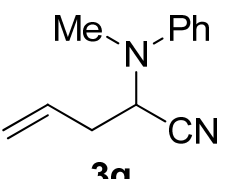

${ }^{13} \mathrm{C} \mathrm{NMR}\left(100 \mathrm{MHz}, \mathrm{CDCl}_{3}\right)$

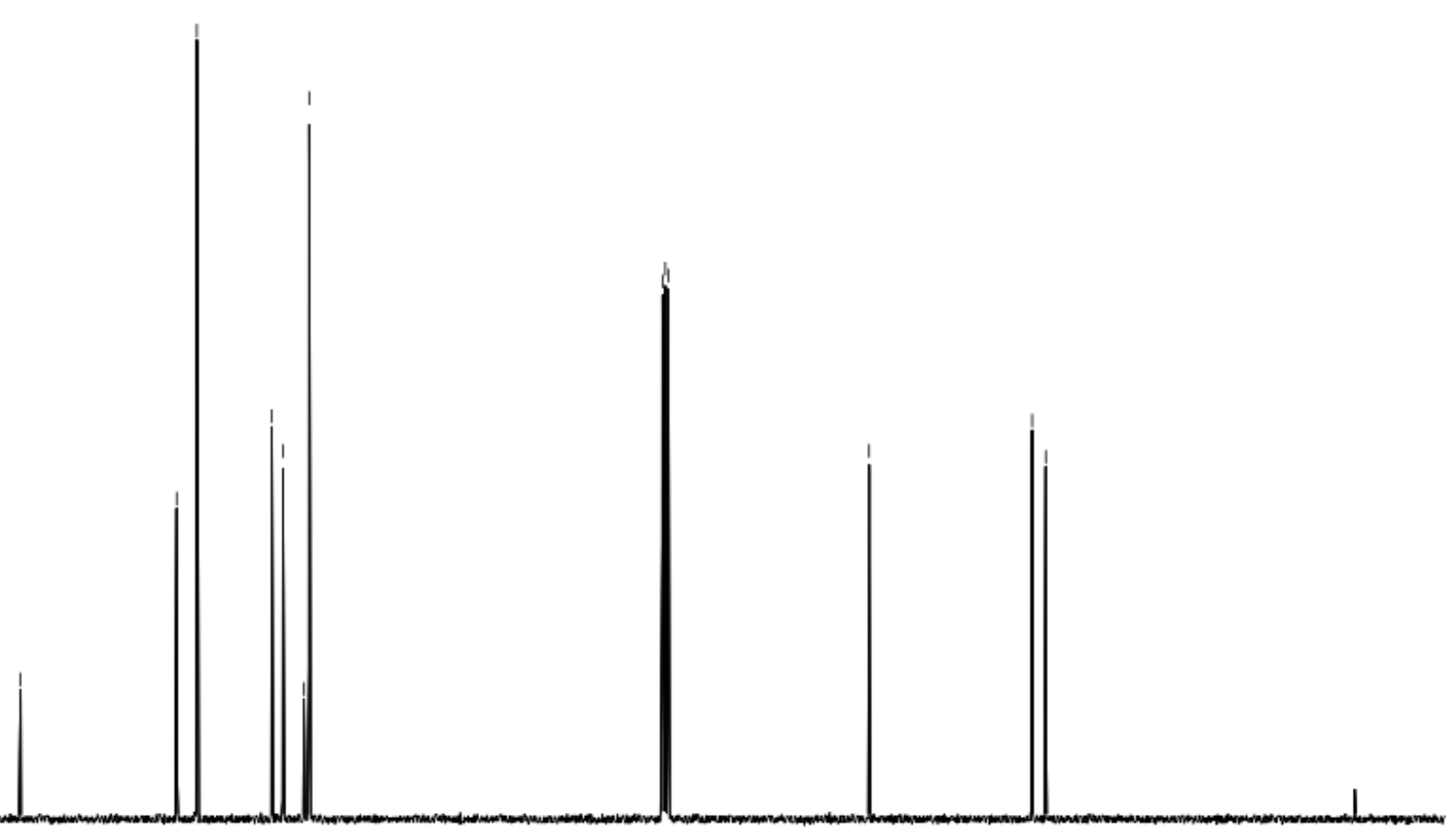

$\begin{array}{lllllllllll}110 & 200 & 190 & 180 & 170 & 160 & 150 & 140 & 130 & 120 & 110 \\ f 1 & (\mathrm{ppm})\end{array}$ 


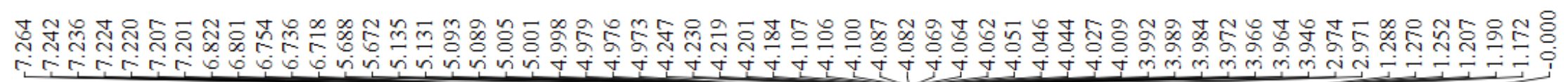

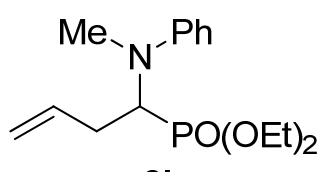

$3 \mathrm{~h}$

${ }^{1} \mathrm{H} \mathrm{NMR}\left(400 \mathrm{MHz}, \mathrm{CDCl}_{3}\right)$

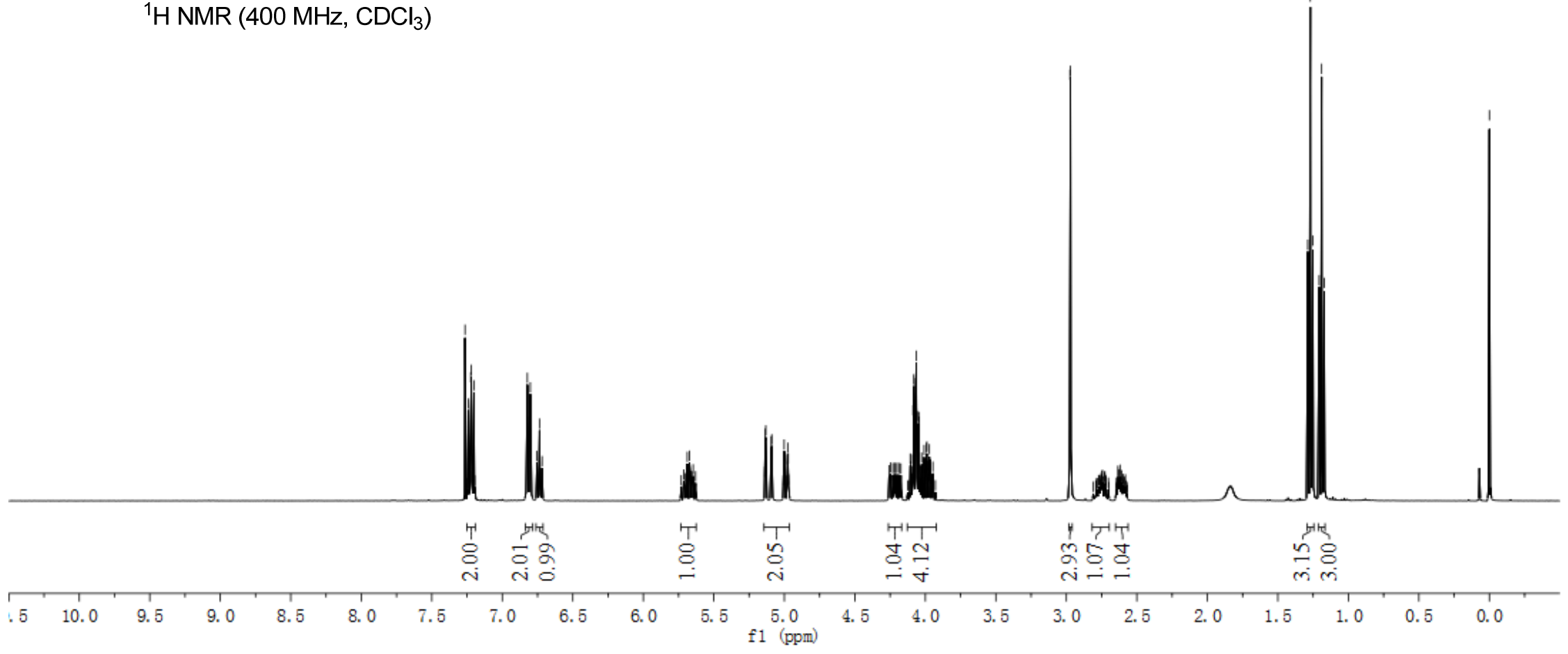




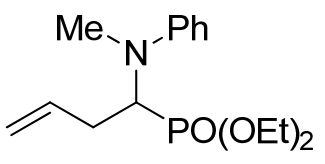

${ }^{13} \mathrm{C}$ NMR (100 MHz, $\left.\mathrm{CDCl}_{3}\right)$

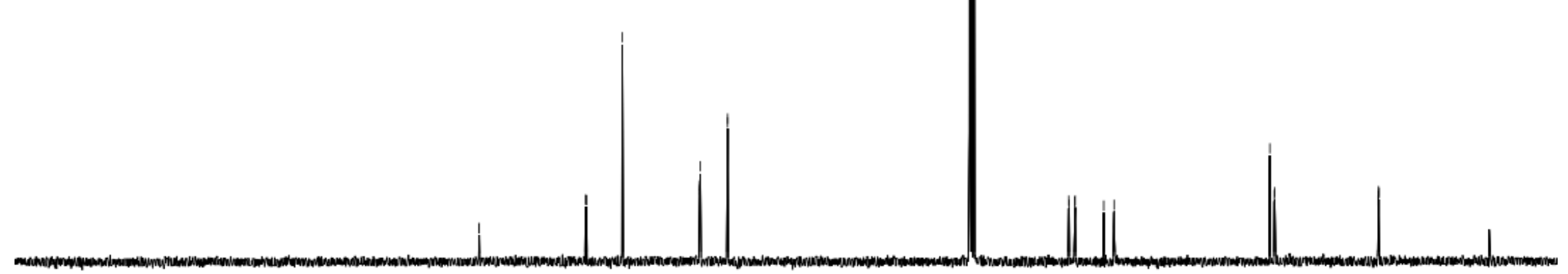

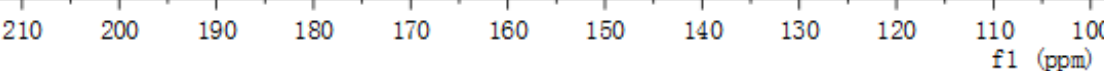




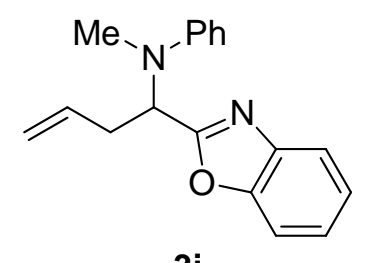

3i

${ }^{1} \mathrm{H} \mathrm{NMR}\left(400 \mathrm{MHz}, \mathrm{CDCl}_{3}\right)$

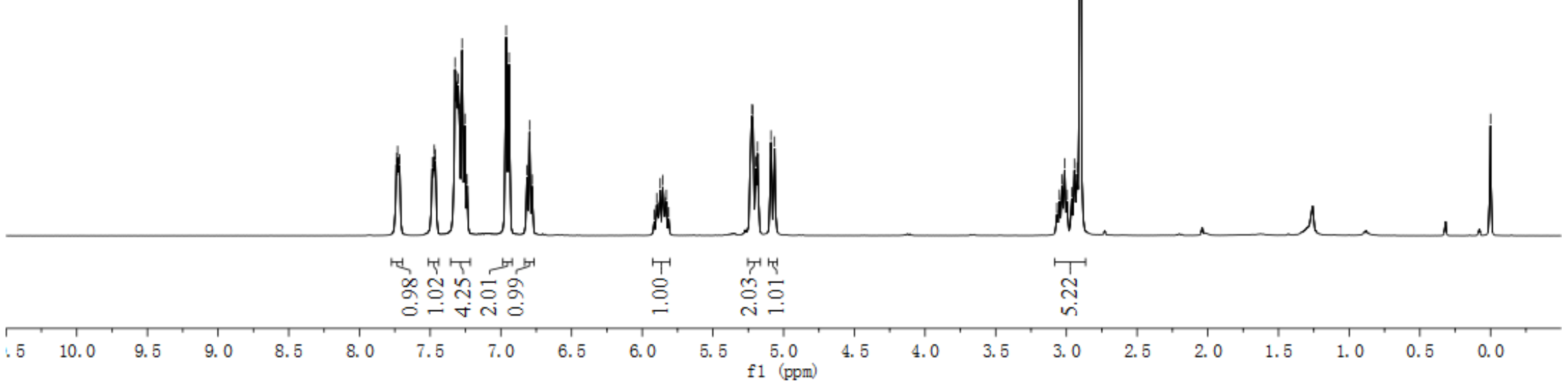




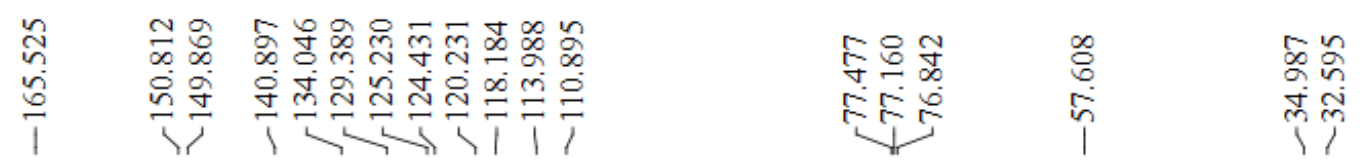

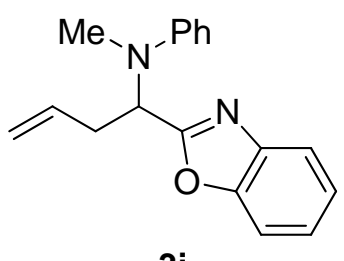

$3 i$

${ }^{13} \mathrm{C}$ NMR $\left(100 \mathrm{MHz}, \mathrm{CDCl}_{3}\right)$

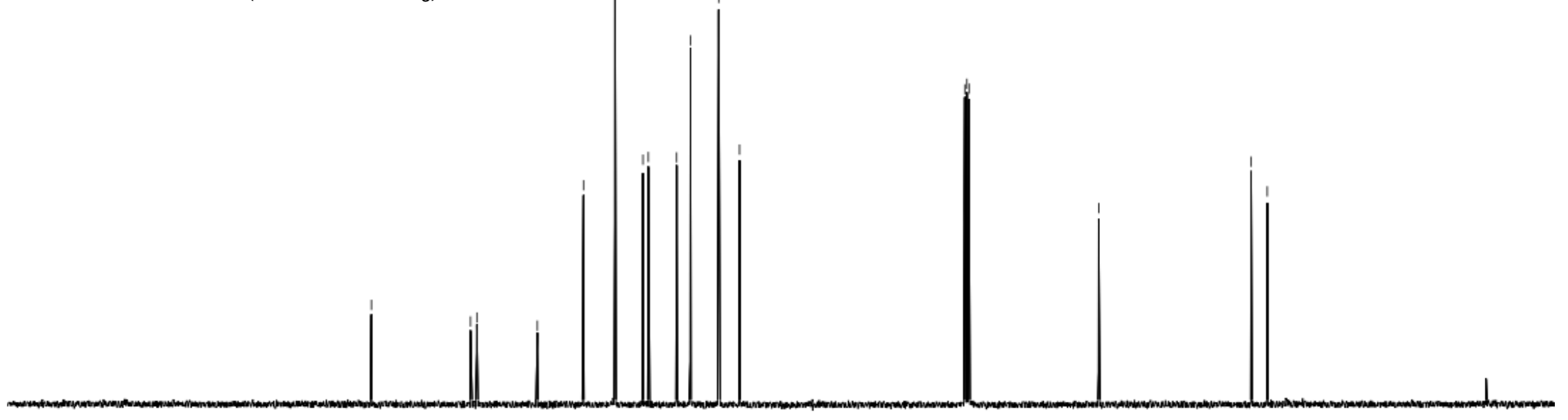

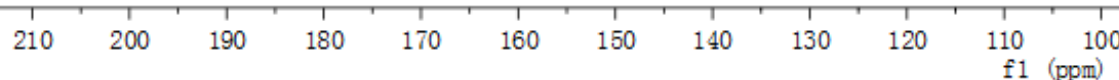




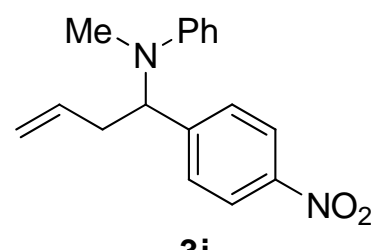

3j

${ }^{1} \mathrm{H}$ NMR (400 MHz, $\mathrm{CDCl}_{3}$ )

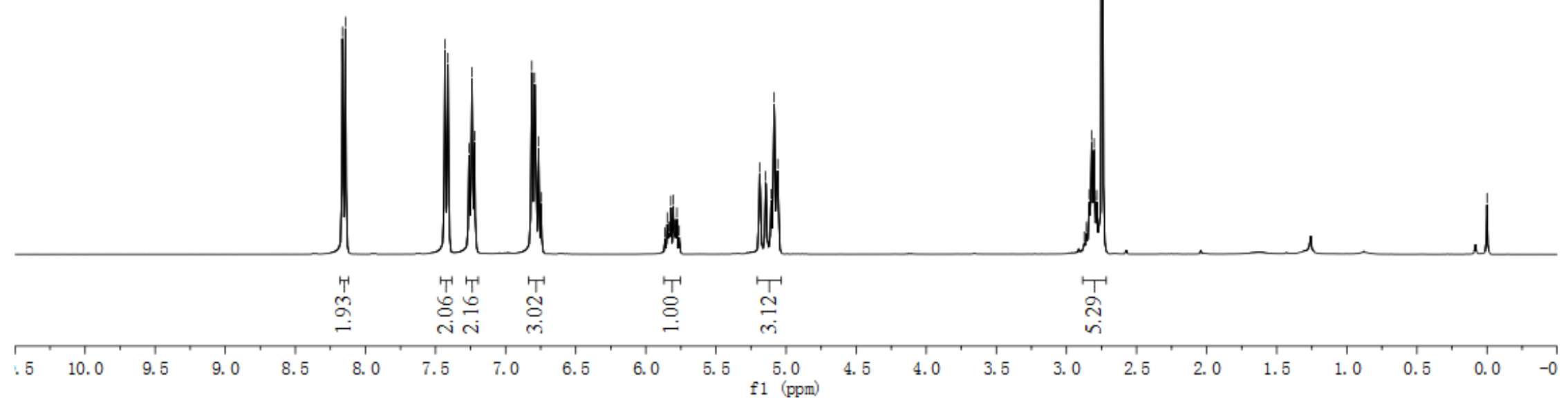




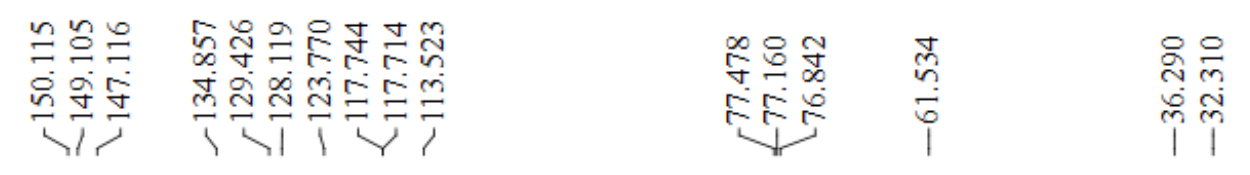

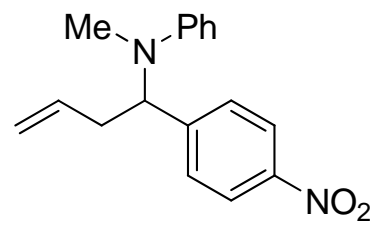

3j

${ }^{13} \mathrm{C}$ NMR $\left(100 \mathrm{MHz}, \mathrm{CDCl}_{3}\right)$

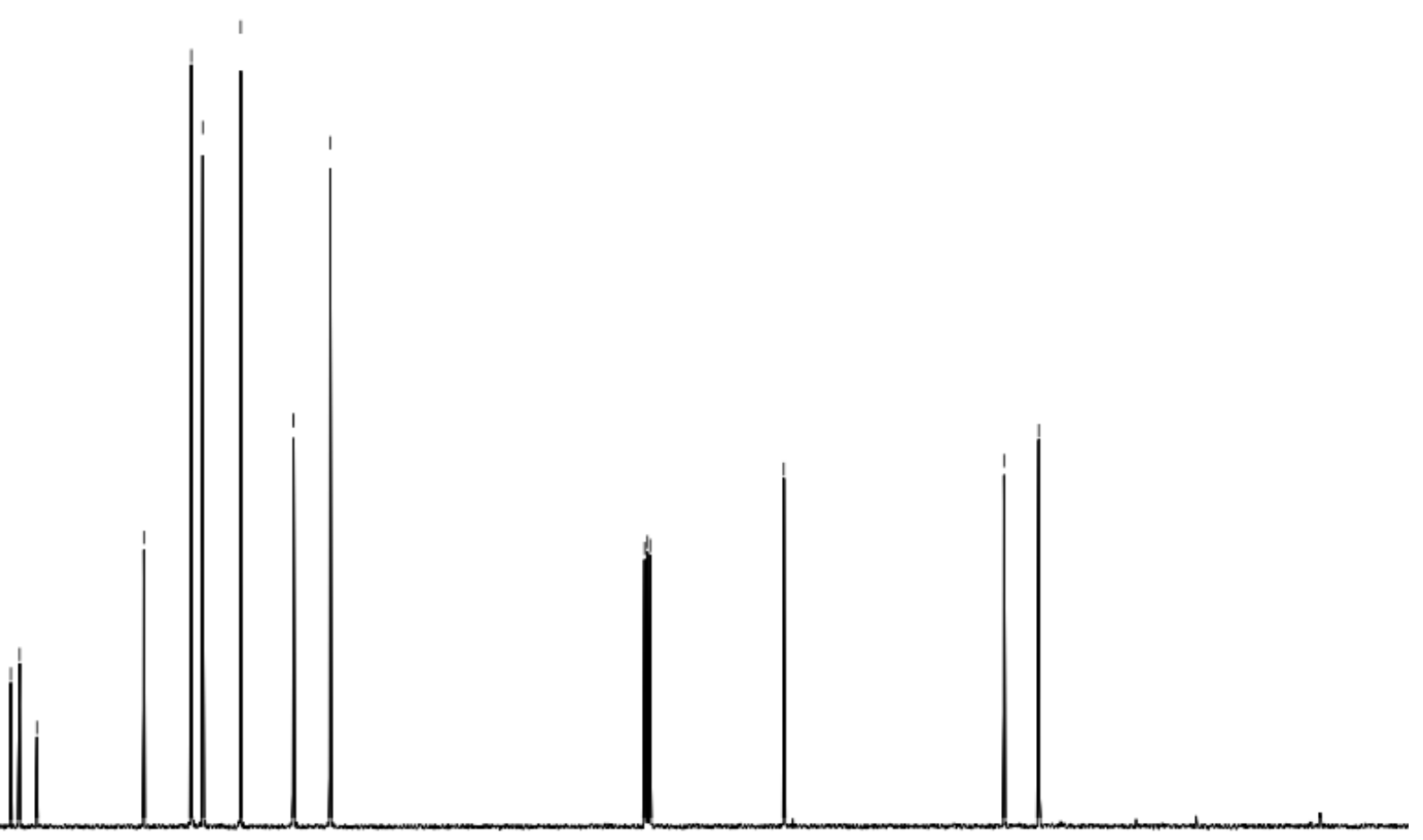

$\begin{array}{llllllllllll}210 & 200 & 190 & 180 & 170 & 160 & 150 & 140 & 130 & 120 & 110 & 100 \\ \mathrm{f} 1 & (\mathrm{ppm})\end{array}$ 


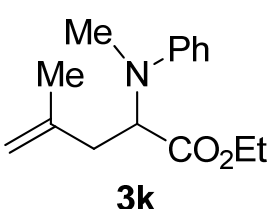

${ }^{1} \mathrm{H} \mathrm{NMR}\left(400 \mathrm{MHz}, \mathrm{CDCl}_{3}\right)$

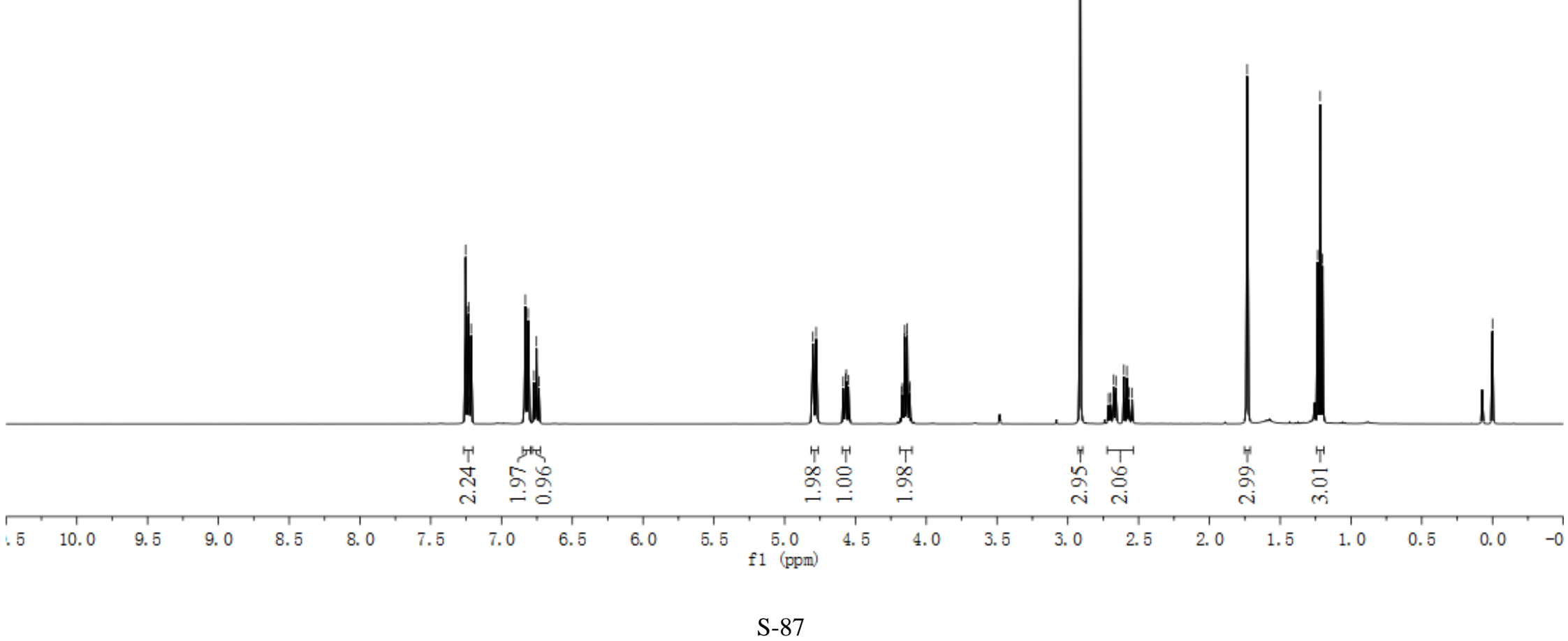




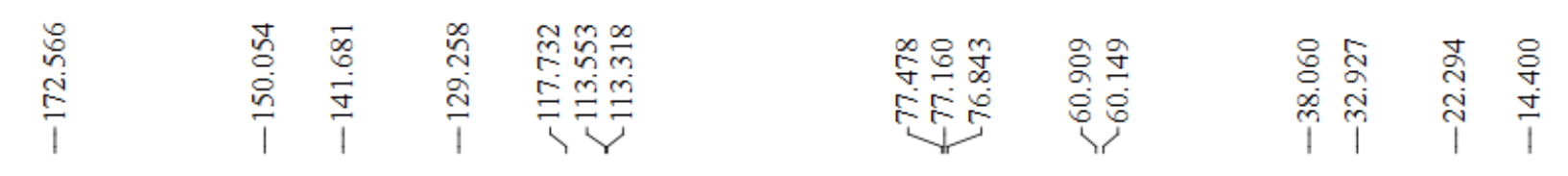

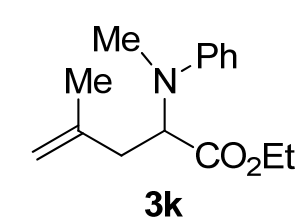

${ }^{13} \mathrm{C} \mathrm{NMR}\left(100 \mathrm{MHz}, \mathrm{CDCl}_{3}\right)$

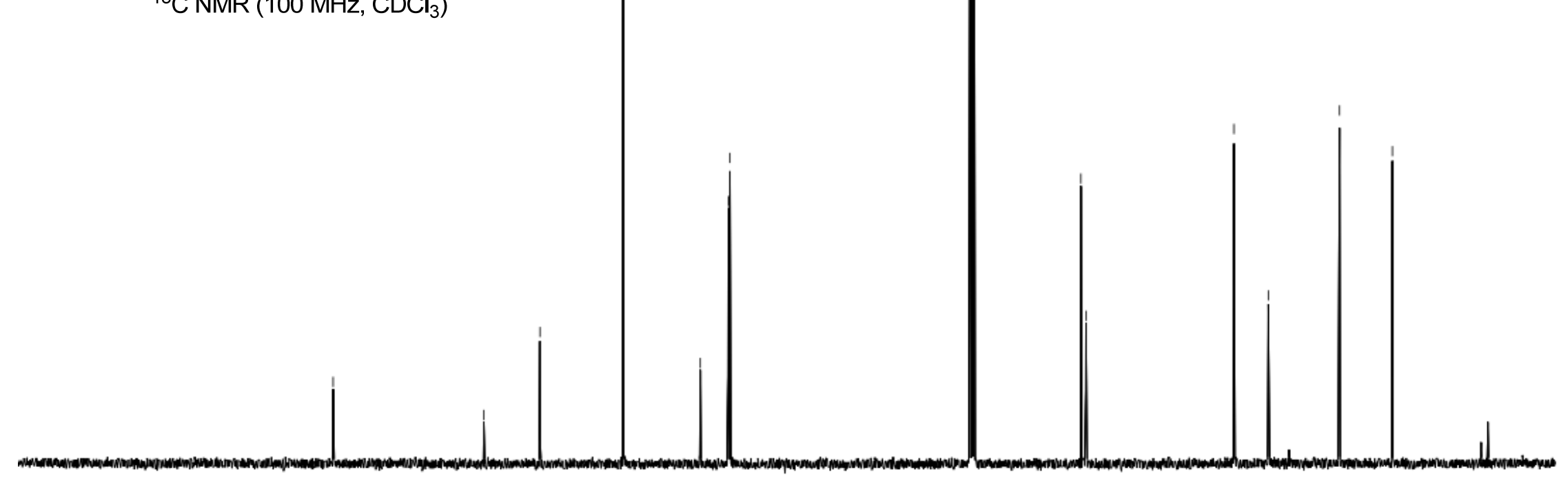

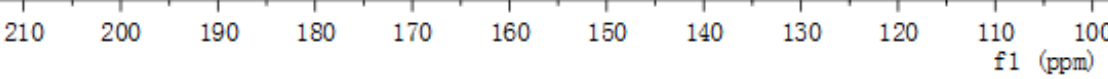




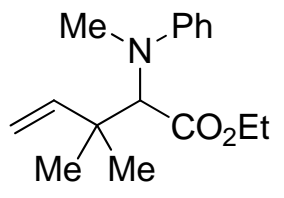

3)

${ }^{1} \mathrm{H}$ NMR $\left(400 \mathrm{MHz}, \mathrm{CDCl}_{3}\right)$

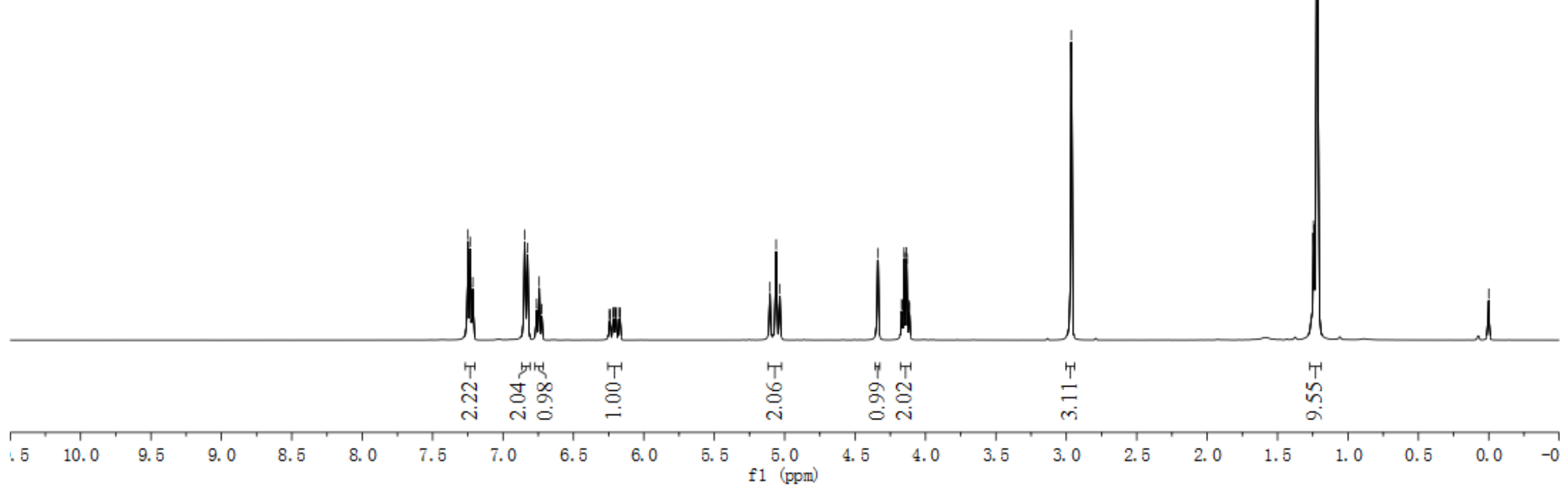




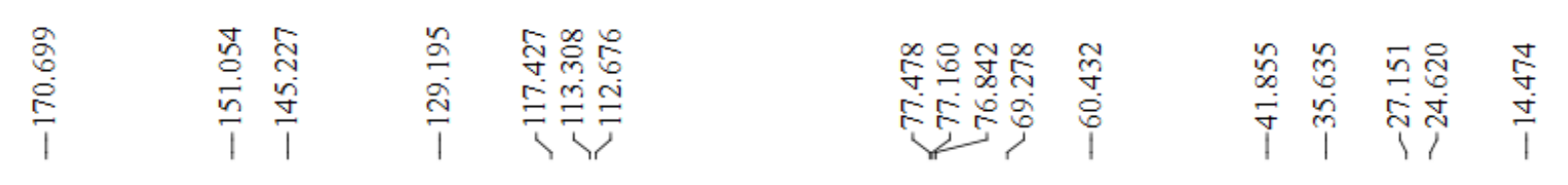

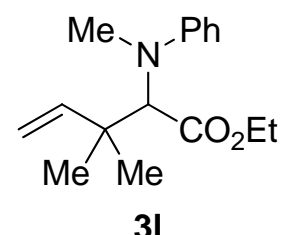

${ }^{13} \mathrm{C}$ NMR $\left(100 \mathrm{MHz}, \mathrm{CDCl}_{3}\right)$

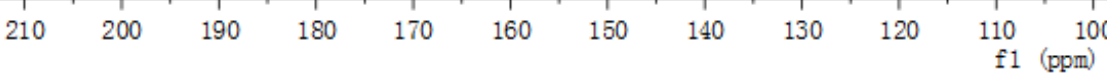




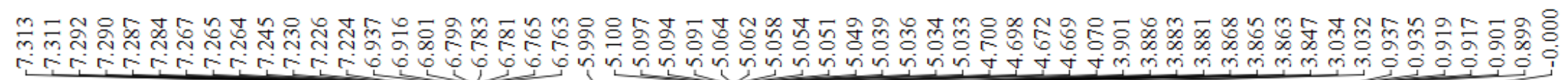

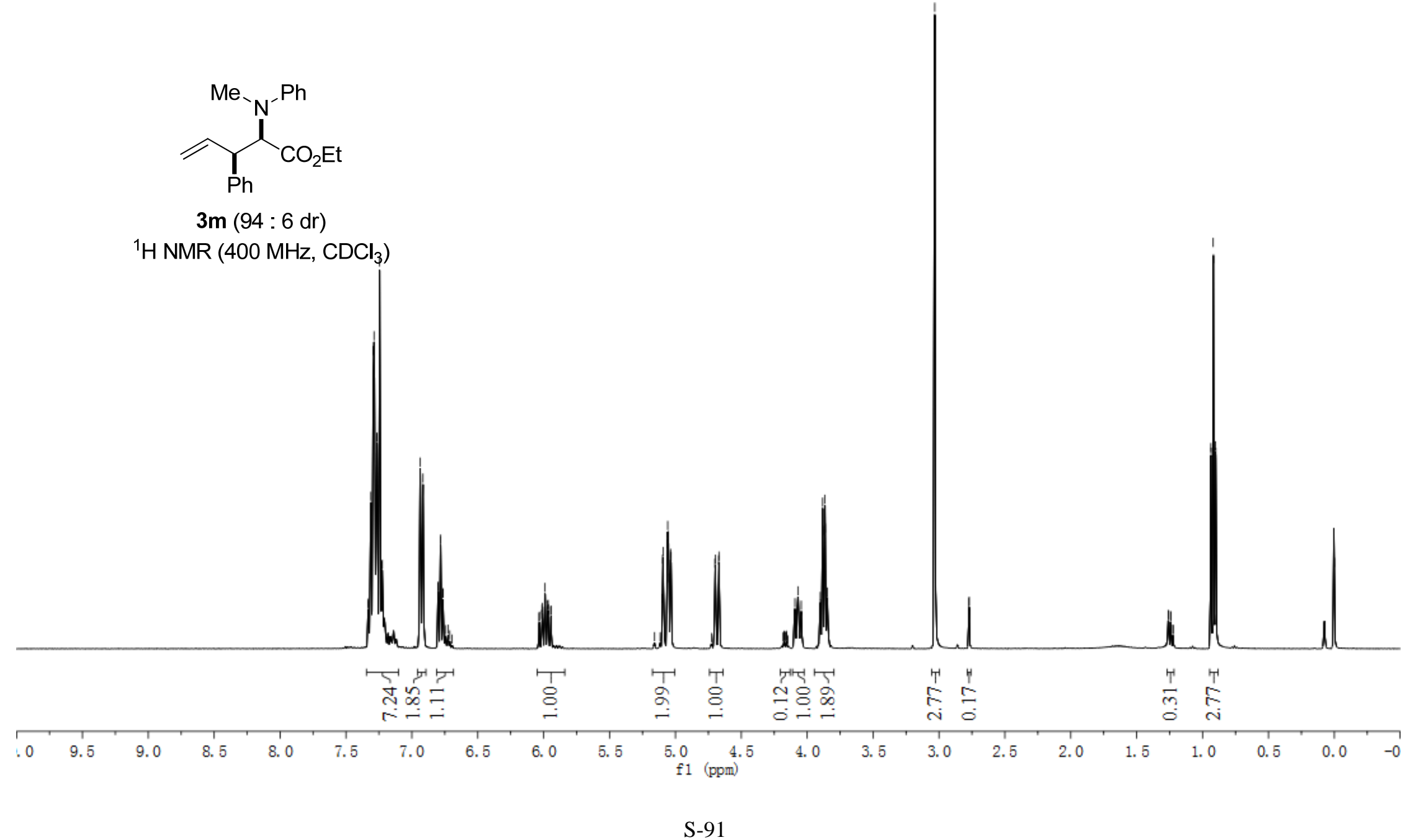




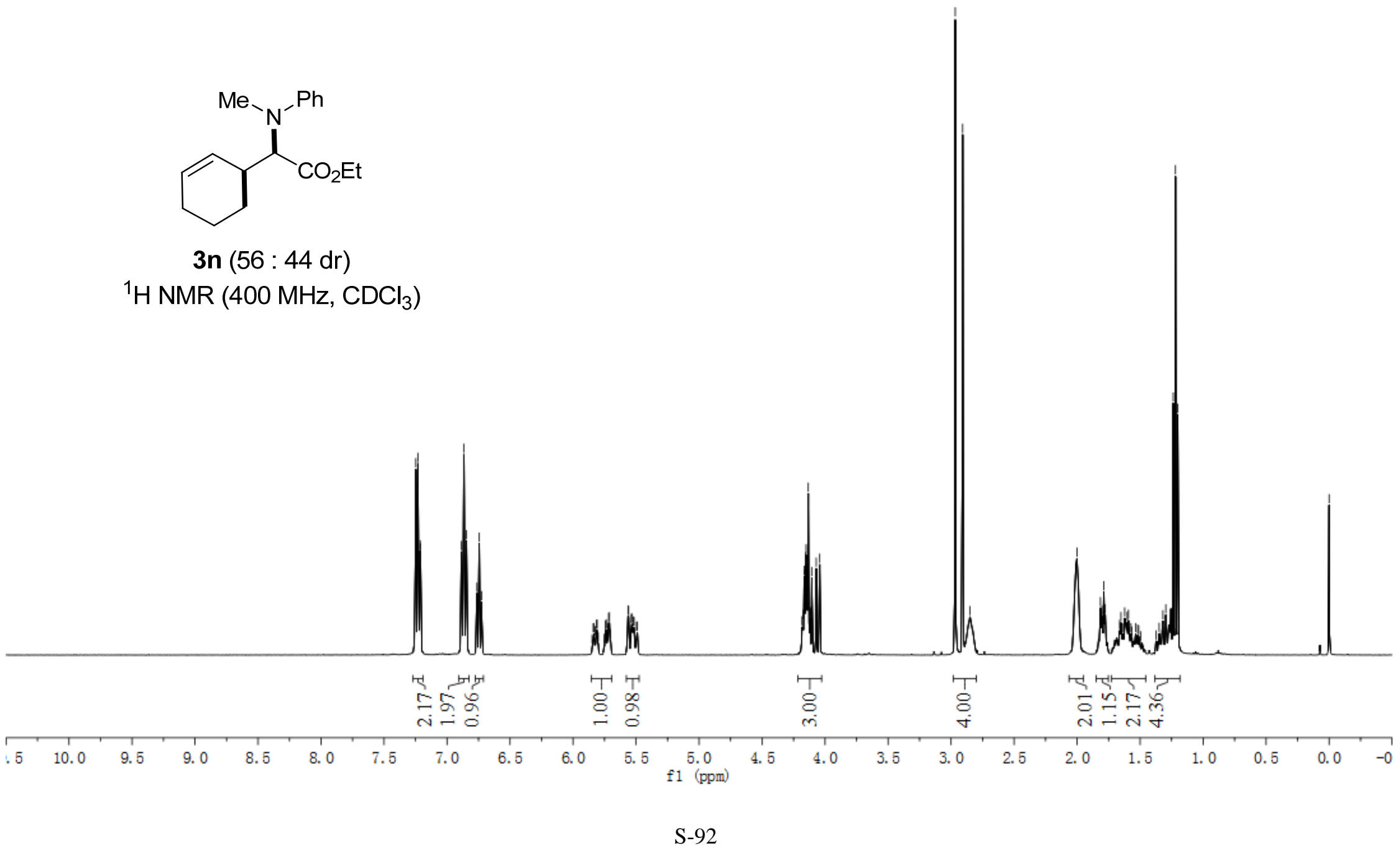




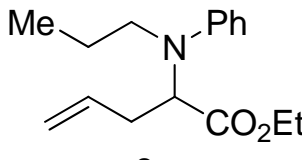

30

${ }^{1} \mathrm{H}$ NMR $\left(400 \mathrm{MHz}, \mathrm{CDCl}_{3}\right)$

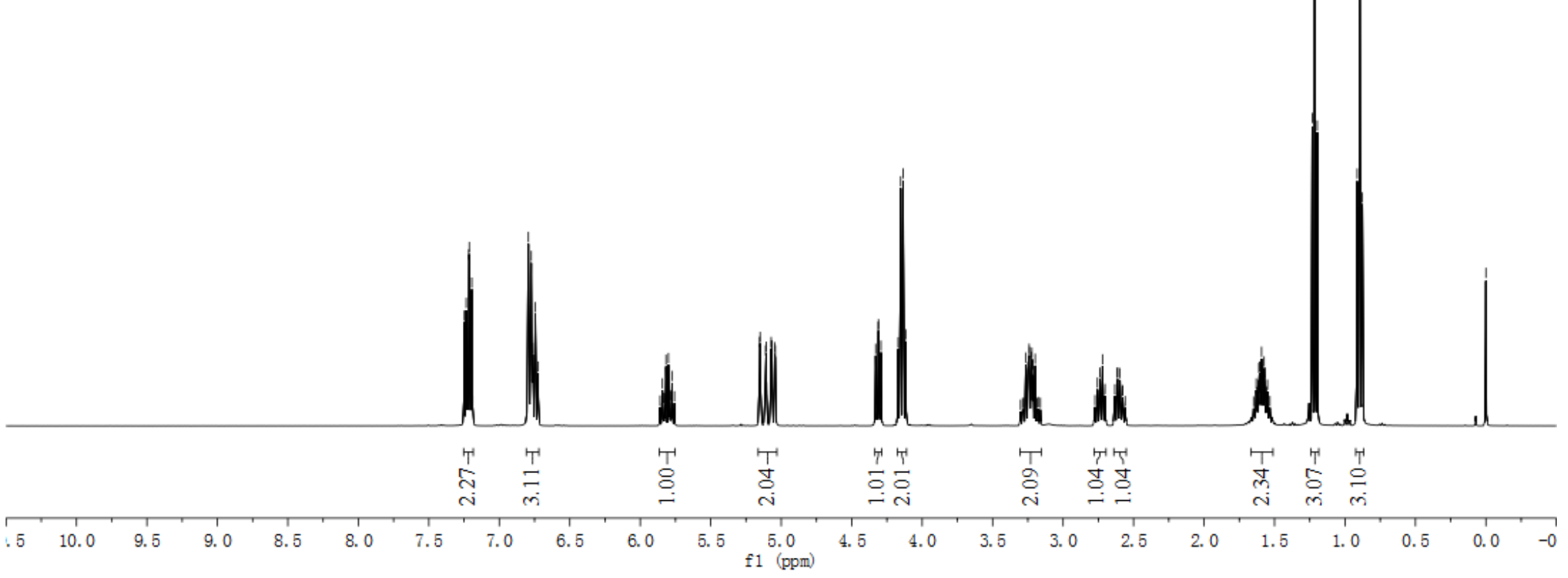




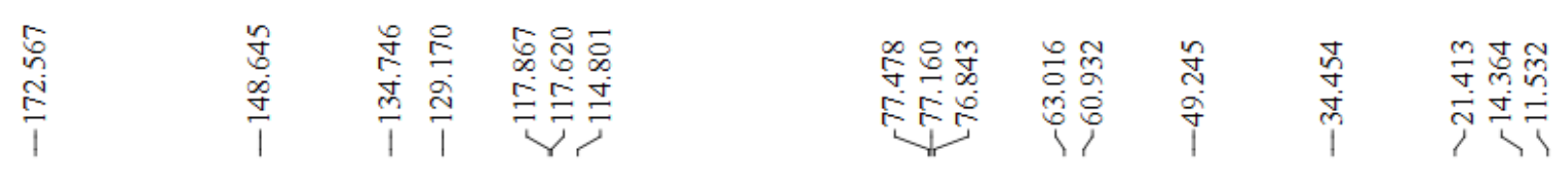

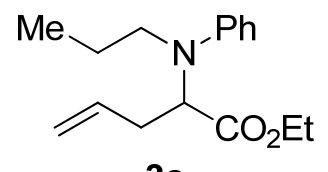

30

${ }^{13} \mathrm{C}$ NMR $\left(100 \mathrm{MHz}, \mathrm{CDCl}_{3}\right)$

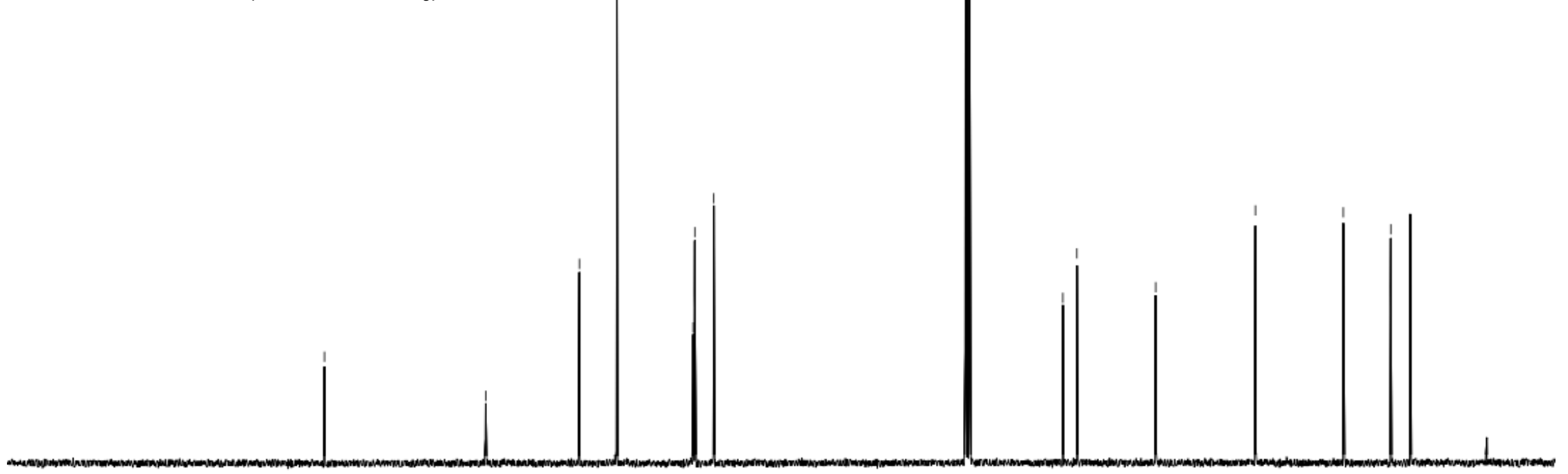

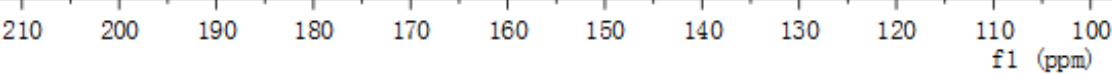




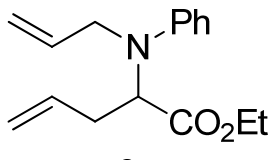

$3 p$

${ }^{1} \mathrm{H}$ NMR $\left(400 \mathrm{MHz}, \mathrm{CDCl}_{3}\right)$

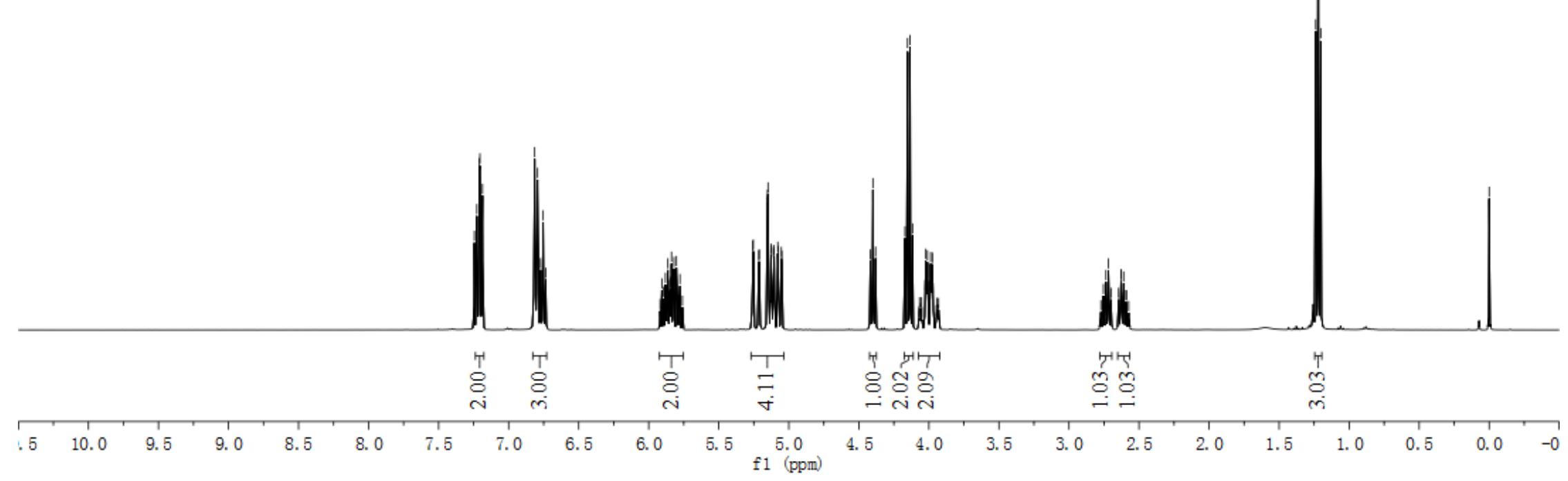




\begin{tabular}{|c|c|c|c|c|}
\hline 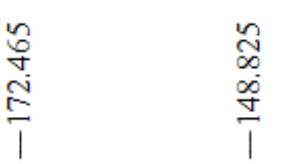 & 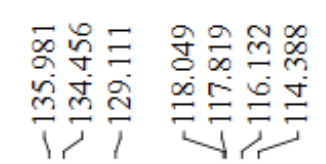 & 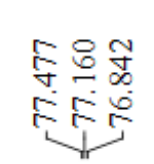 & $\begin{array}{l}\text { हैa } \\
00 \\
00 \\
11\end{array}$ & $\frac{1}{\substack{n \\
0}}$ \\
\hline
\end{tabular}

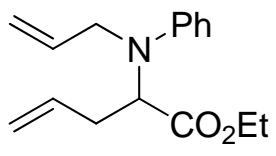

$3 p$

${ }^{13} \mathrm{C}$ NMR $\left(100 \mathrm{MHz}, \mathrm{CDCl}_{3}\right)$

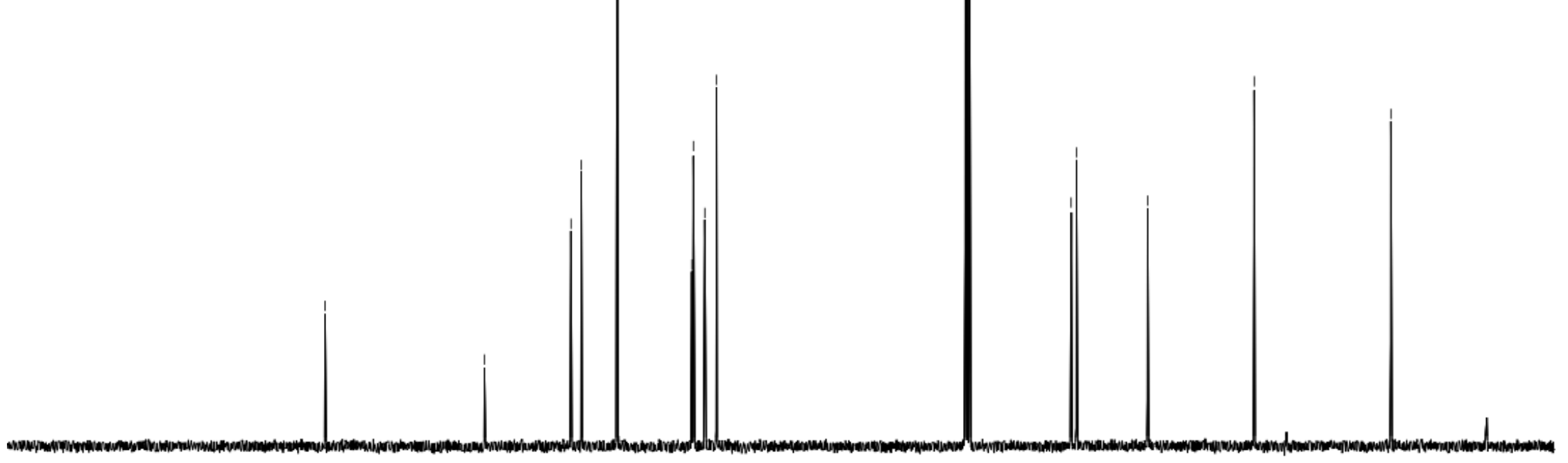

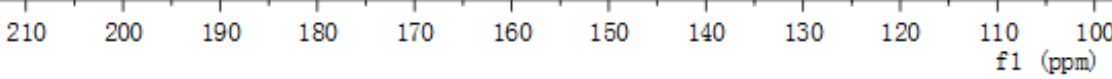




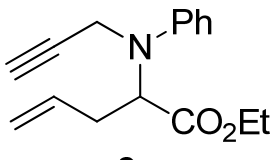

$3 q$

${ }^{1} \mathrm{H}$ NMR $\left(400 \mathrm{MHz}, \mathrm{CDCl}_{3}\right)$

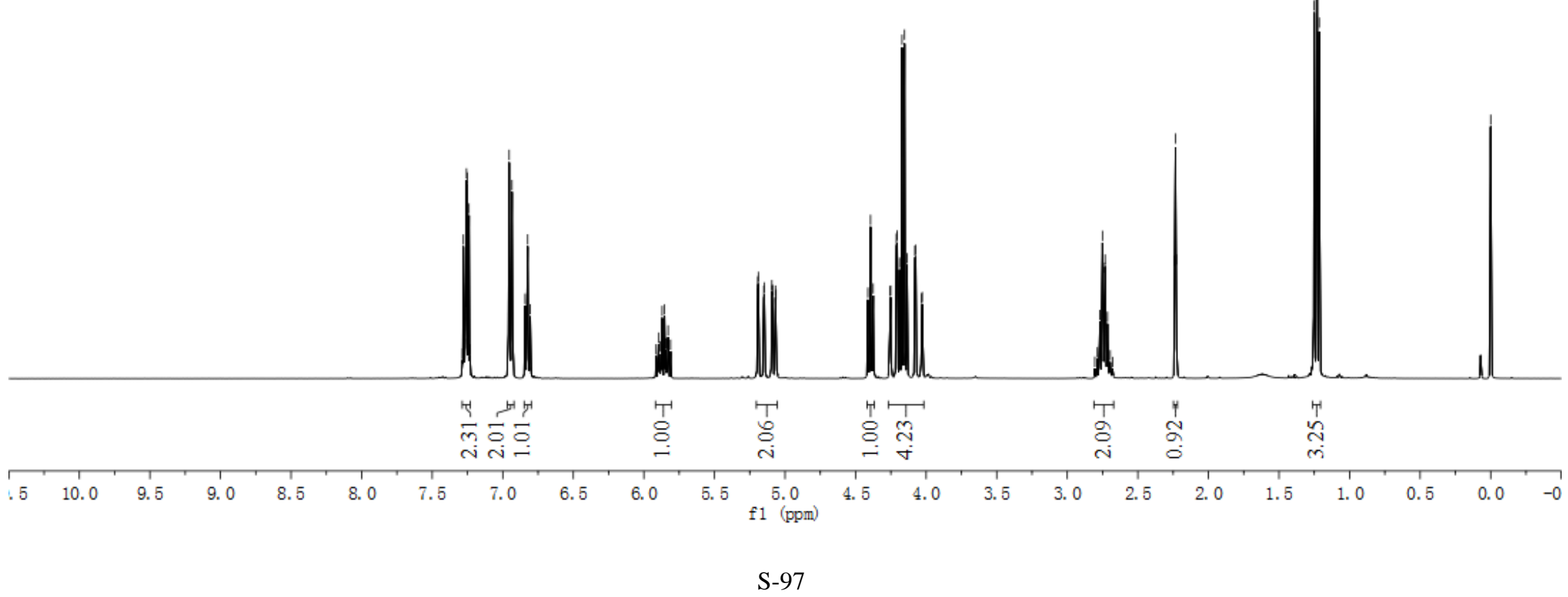




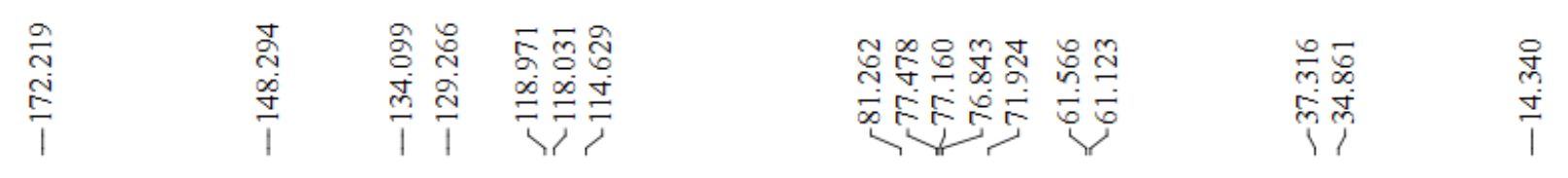

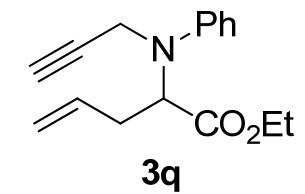

${ }^{13} \mathrm{C}$ NMR $\left(100 \mathrm{MHz}, \mathrm{CDCl}_{3}\right)$

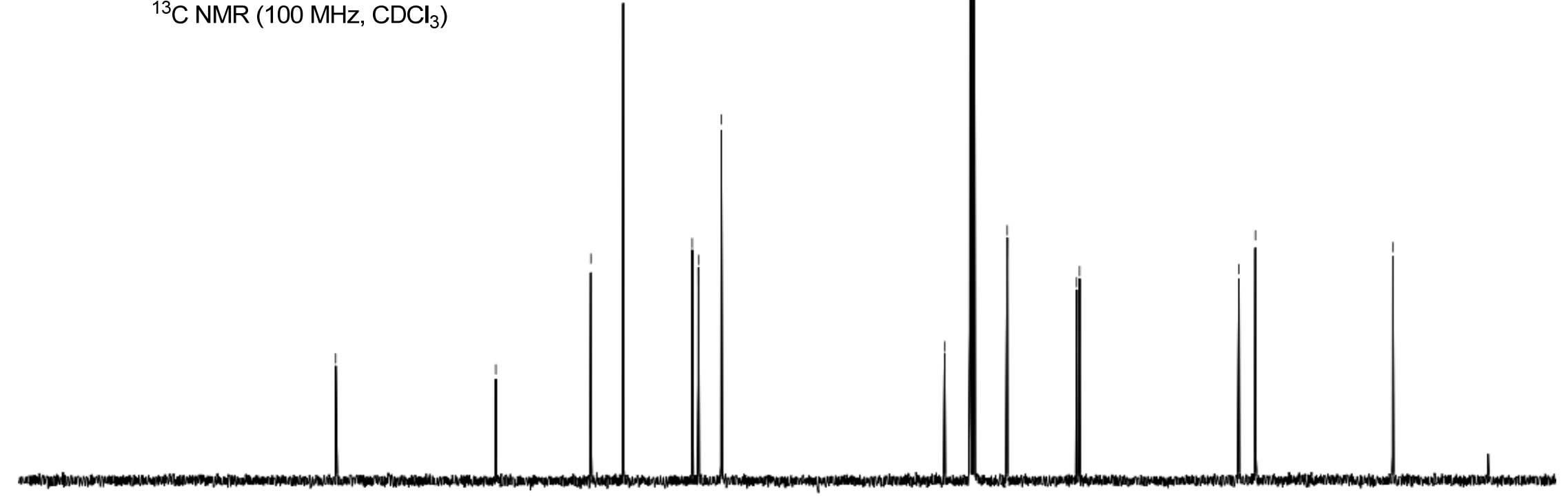

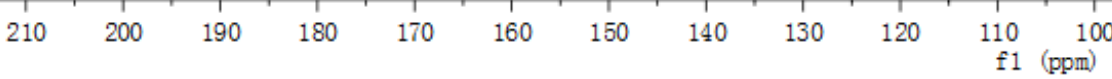




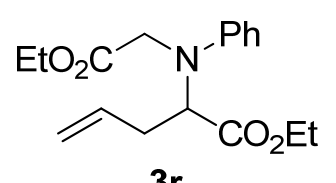

${ }^{1} \mathrm{H} \mathrm{NMR}\left(400 \mathrm{MHz}, \mathrm{CDCl}_{3}\right)$

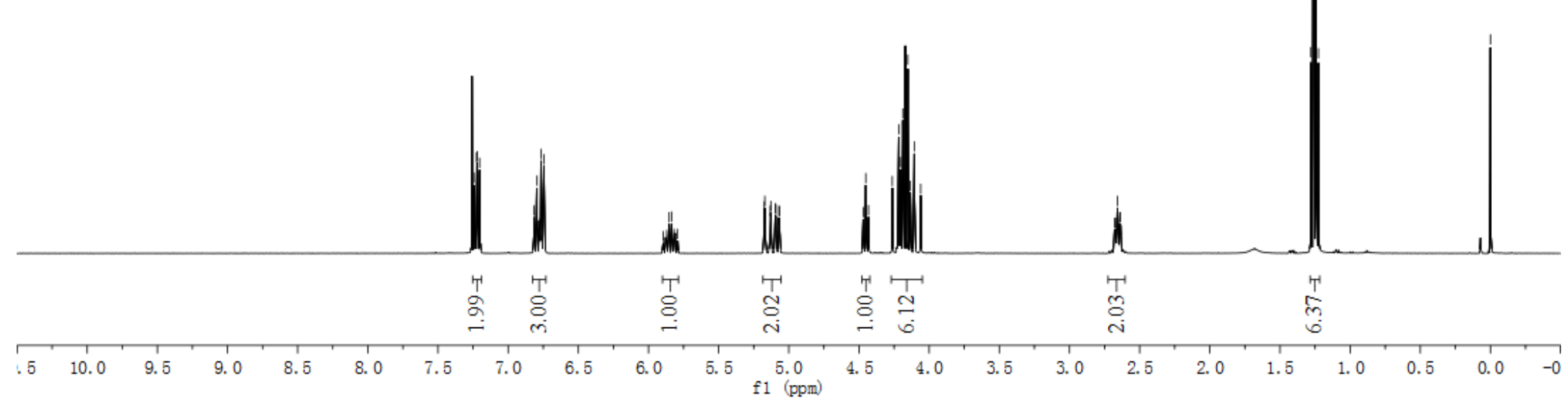




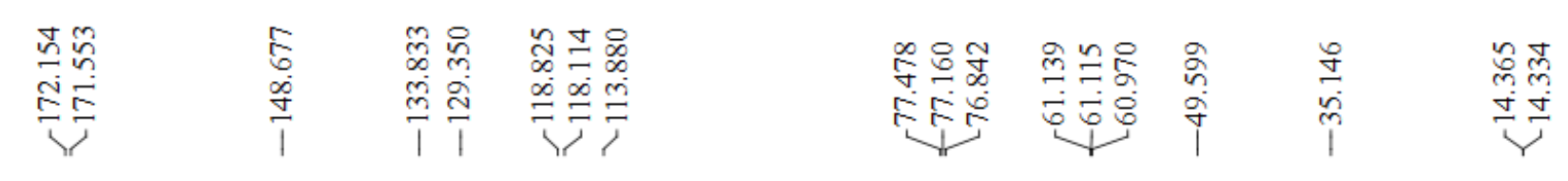

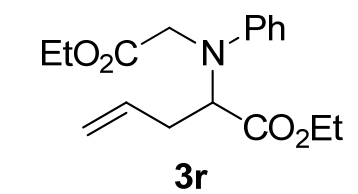

${ }^{13} \mathrm{C}$ NMR (100 MHz, $\mathrm{CDCl}_{3}$ )
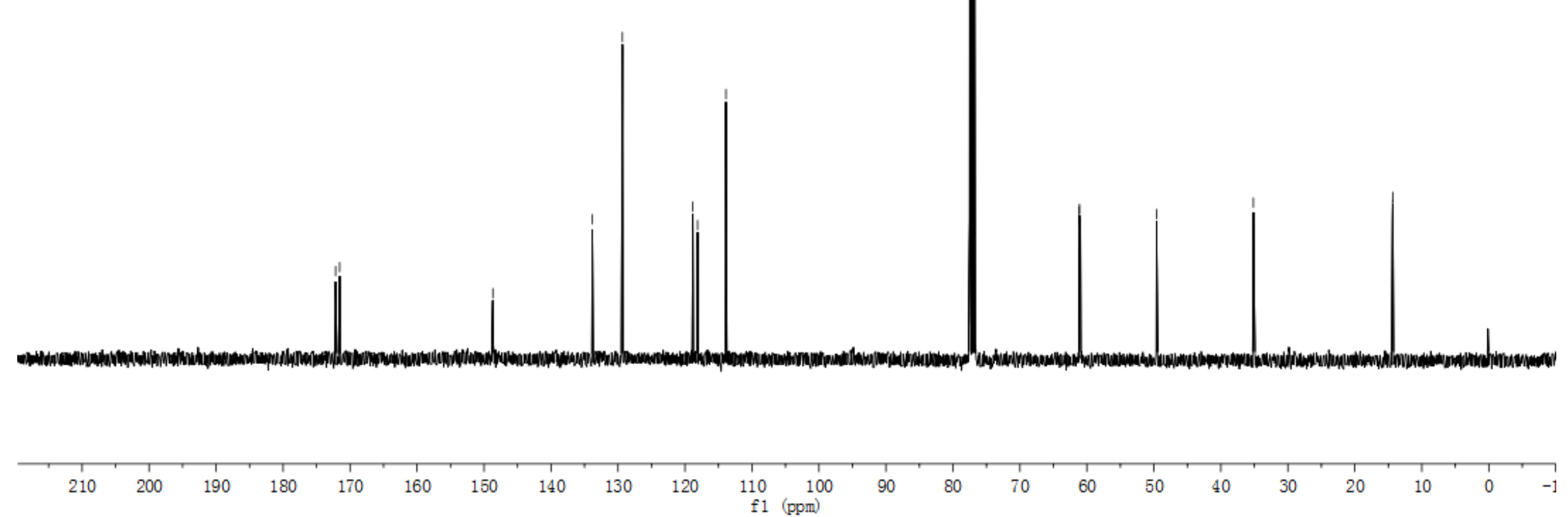

S-100 


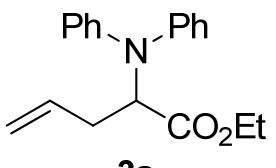

3s

${ }^{1} \mathrm{H}$ NMR $\left(400 \mathrm{MHz}, \mathrm{CDCl}_{3}\right)$

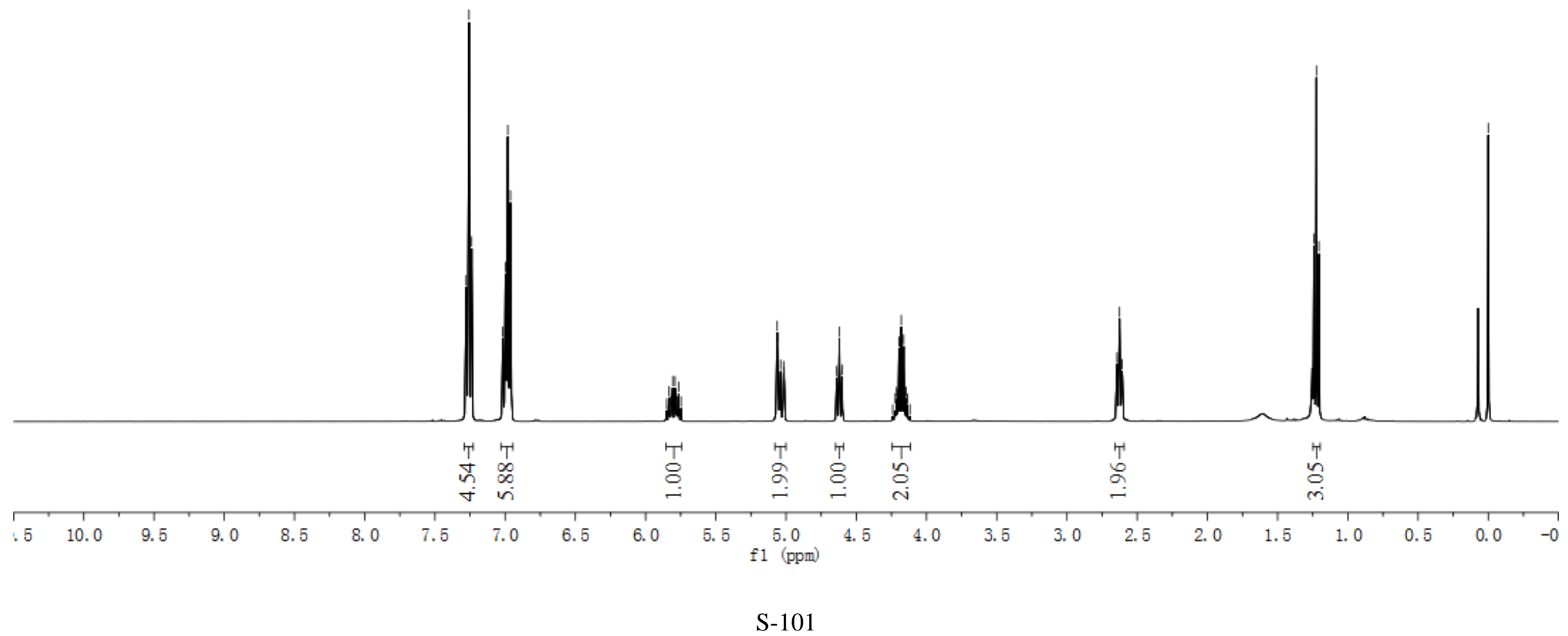




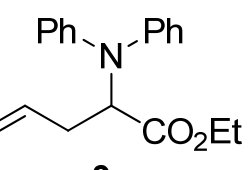

${ }^{13} \mathrm{C}$ NMR (100 MHz, $\mathrm{CDCl}_{3}$ )

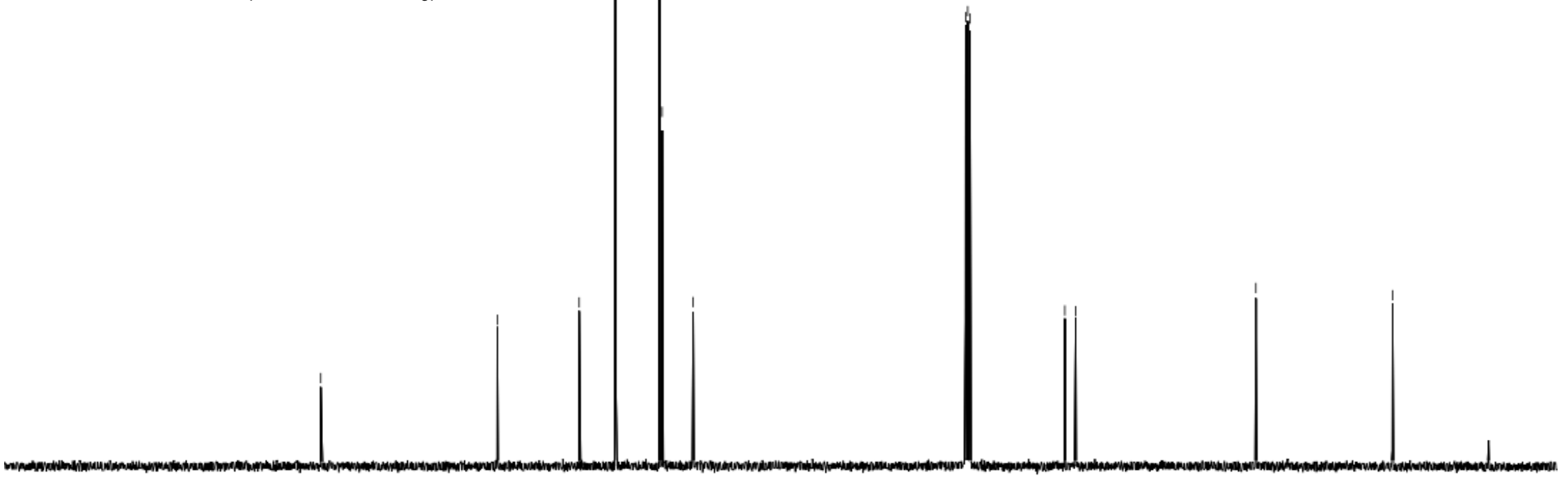




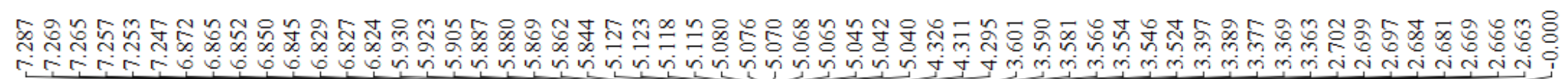

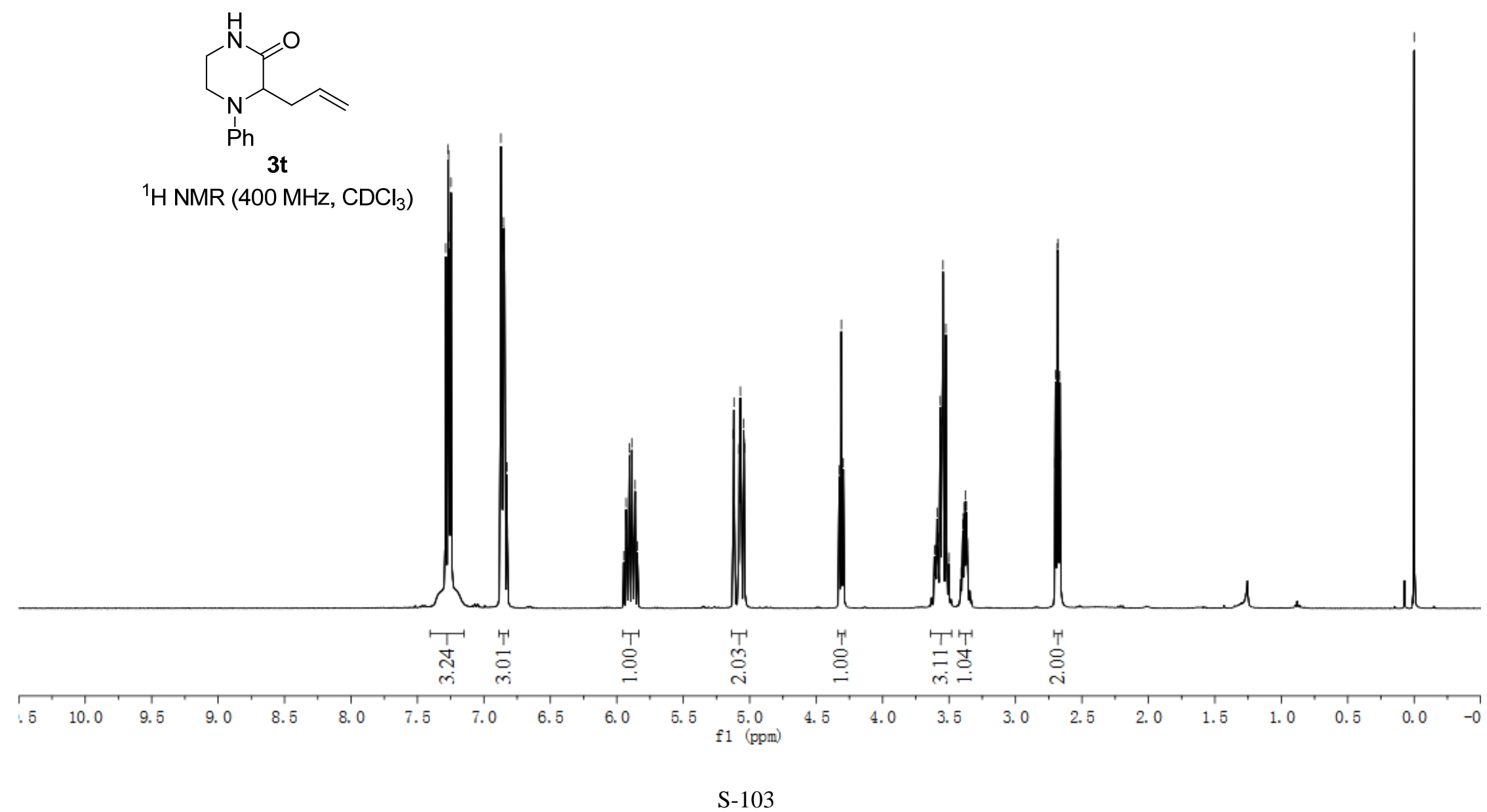




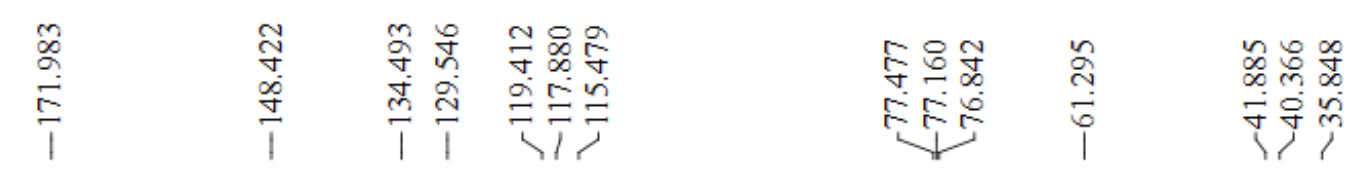

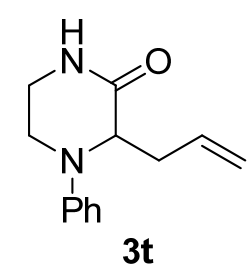

${ }^{13} \mathrm{C} \mathrm{NMR}\left(100 \mathrm{MHz}, \mathrm{CDCl}_{3}\right)$

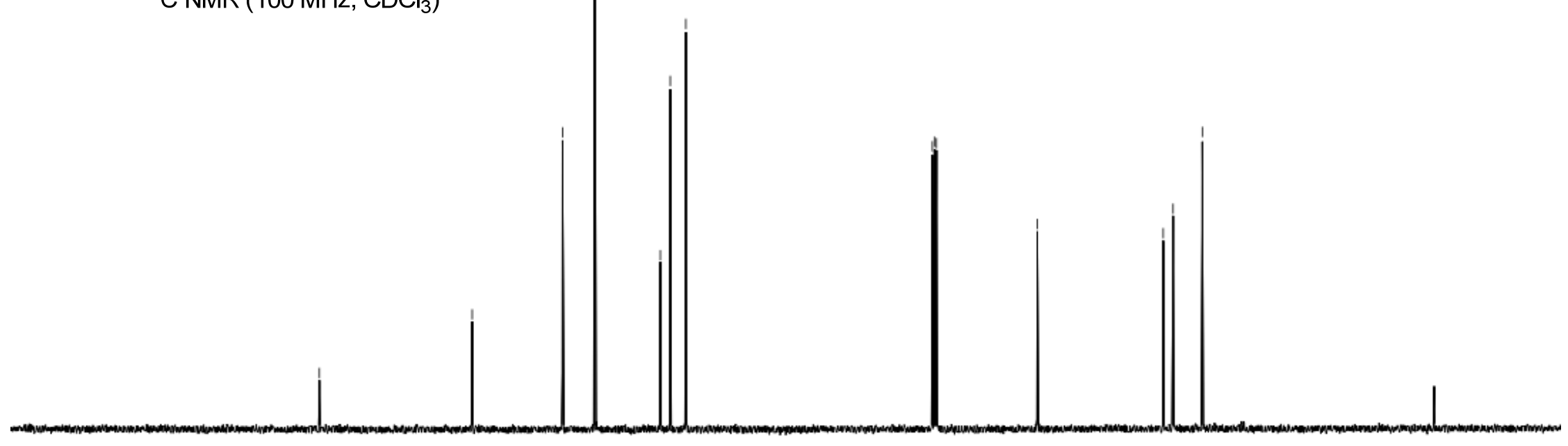

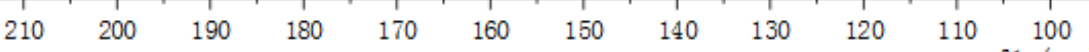

f1 1 (ppm)

S-104 


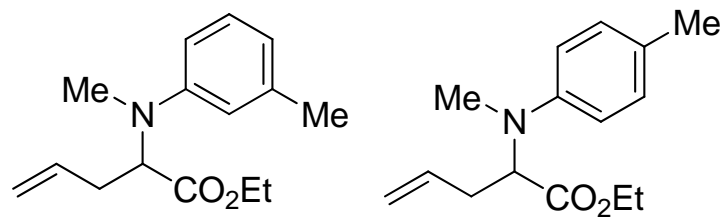

$3 \mathbf{u}$

3u'

${ }^{1} \mathrm{H}$ NMR $\left(400 \mathrm{MHz}, \mathrm{CDCl}_{3}\right)$

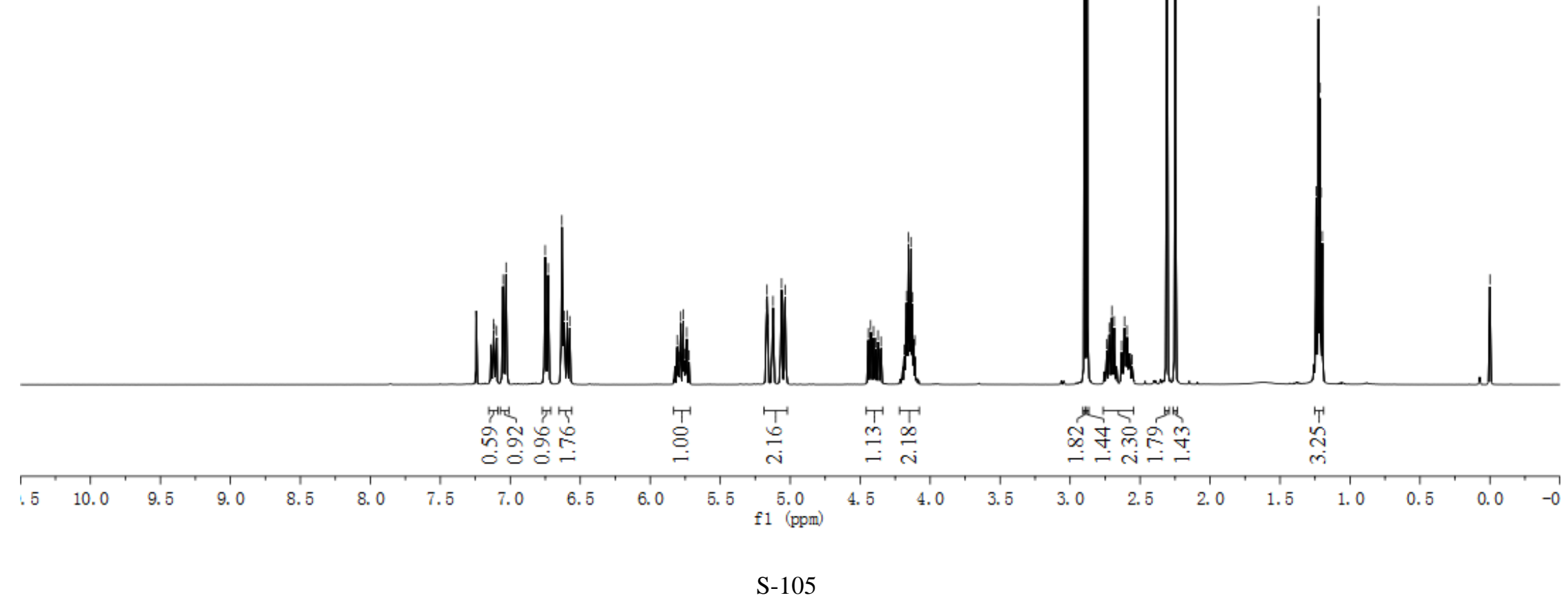




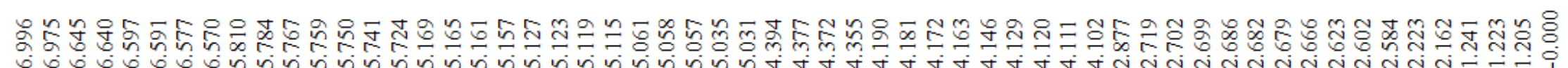

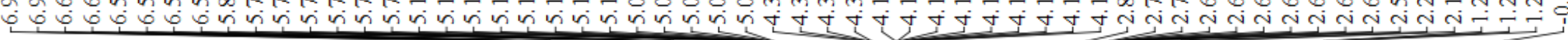

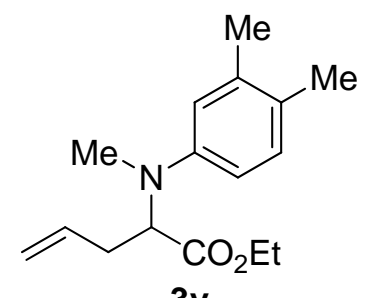

${ }^{1} \mathrm{H} \mathrm{NMR}\left(400 \mathrm{MHz}, \mathrm{CDCl}_{3}\right)$

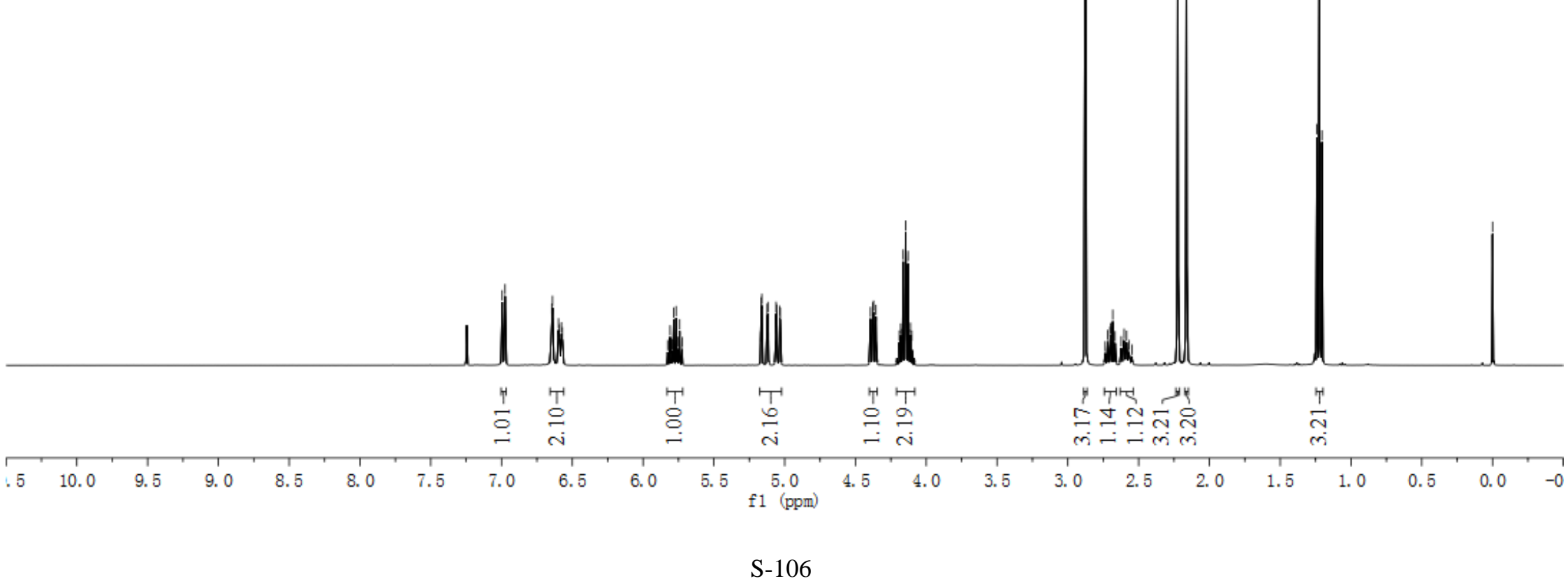




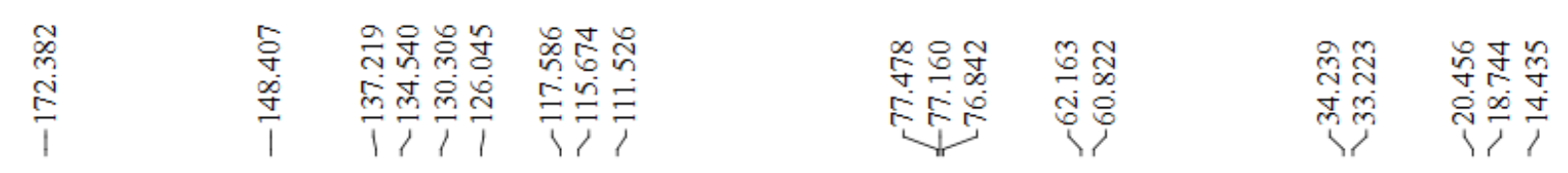

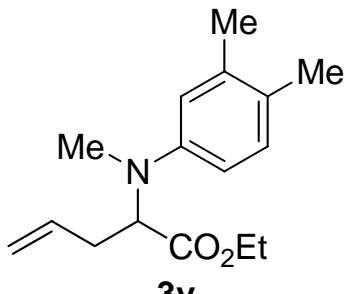

${ }^{13} \mathrm{C}$ NMR $\left(100 \mathrm{MHz}, \mathrm{CDCl}_{3}\right)$

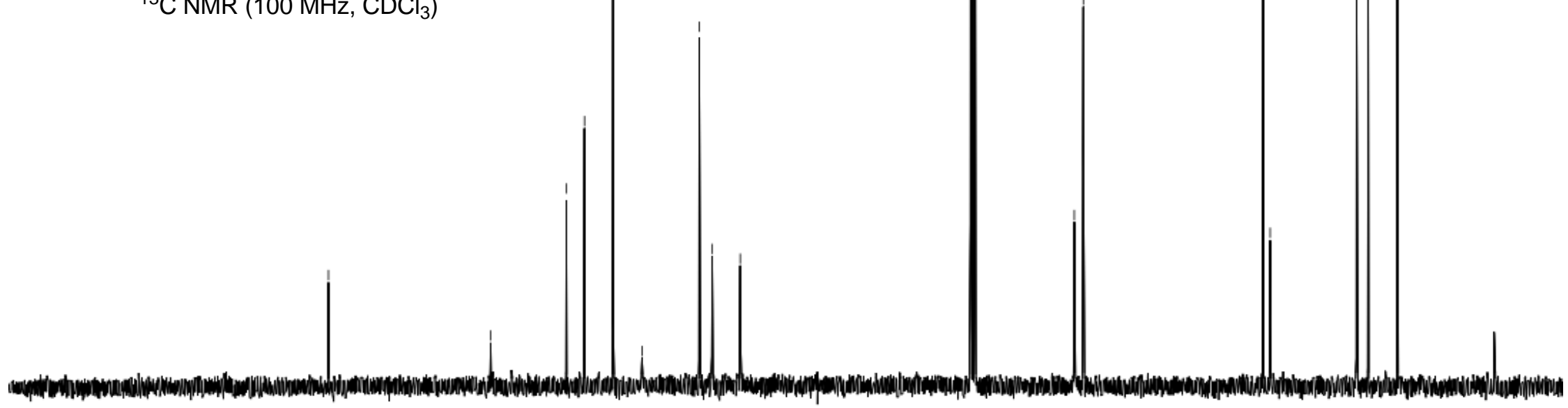

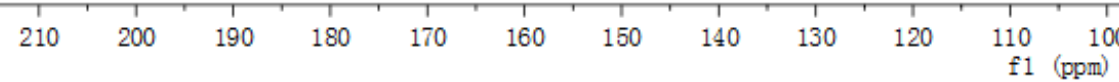




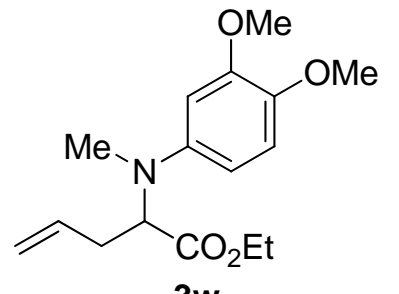

${ }^{1} \mathrm{H} \mathrm{NMR}\left(400 \mathrm{MHz}, \mathrm{CDCl}_{3}\right)$

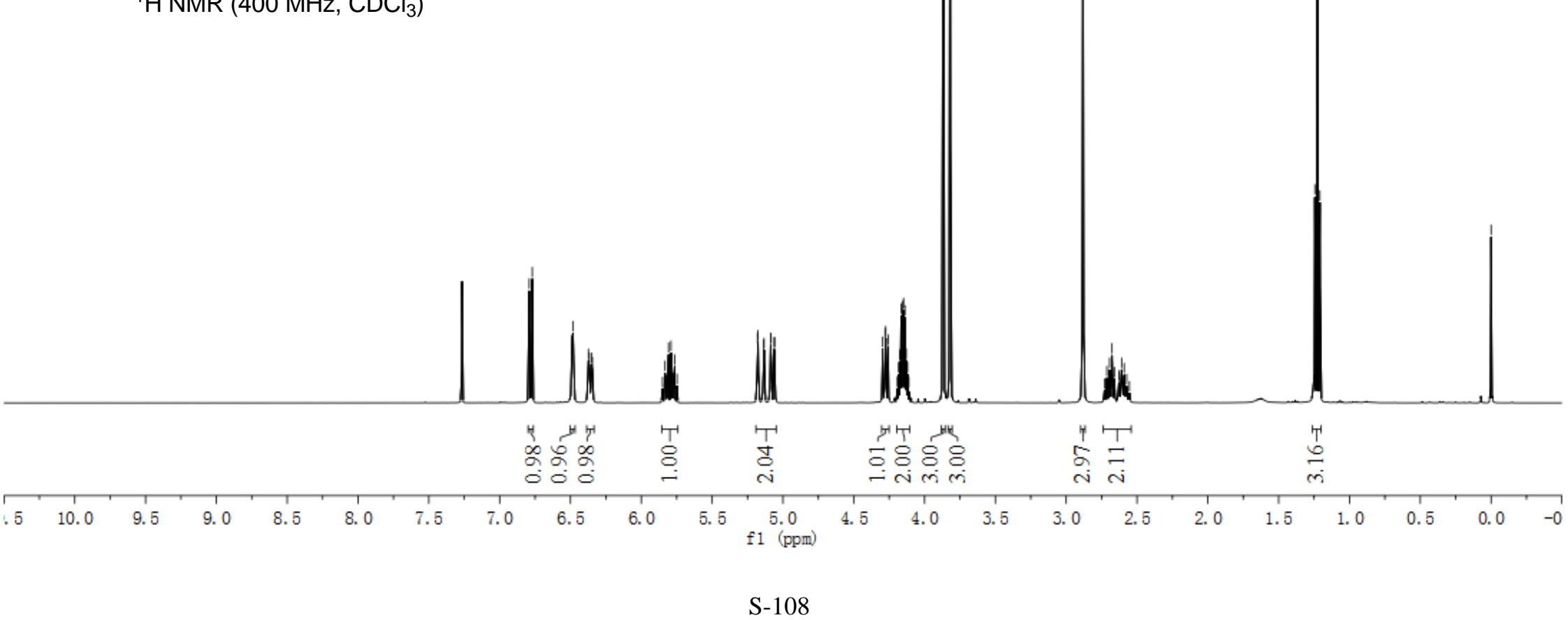




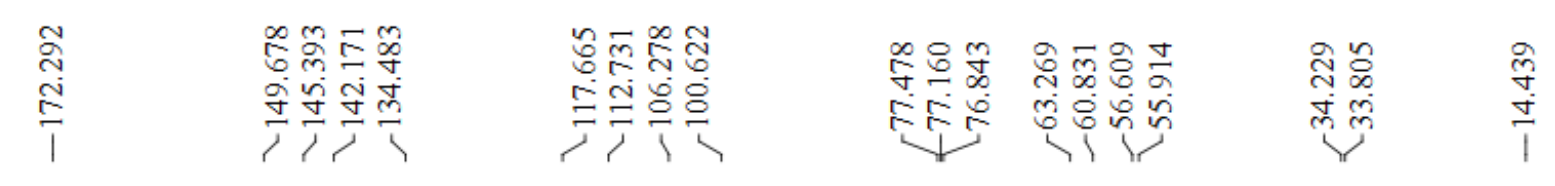

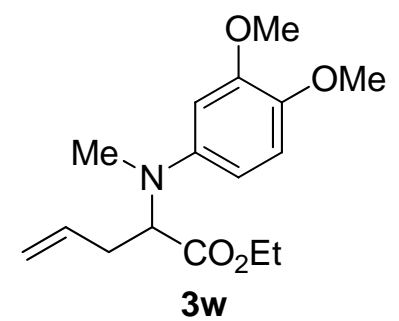

${ }^{13} \mathrm{C} \mathrm{NMR}\left(100 \mathrm{MHz}, \mathrm{CDCl}_{3}\right)$

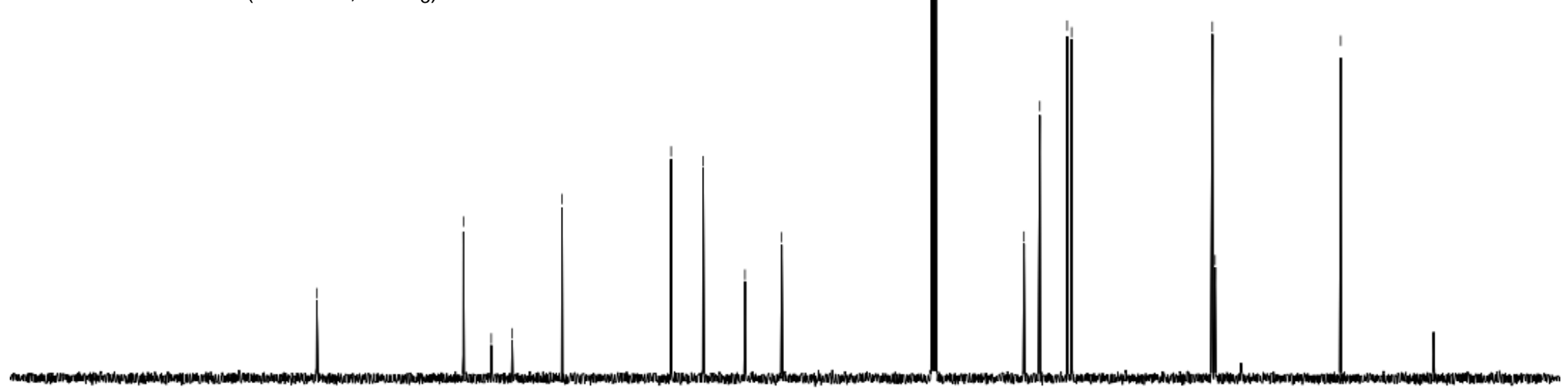

$\begin{array}{llllllllllll}210 & 200 & 190 & 180 & 170 & 160 & 150 & 140 & 130 & 120 & 110 & 100\end{array}$

f1 (ppm)

80

60

50

$40 \quad 30$

20

10

S-109 


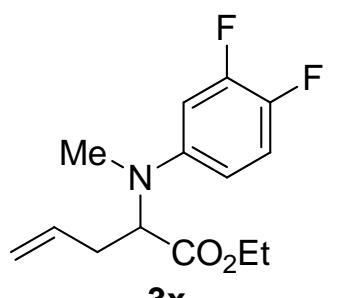

${ }^{1} \mathrm{H}$ NMR $\left(400 \mathrm{MHz}, \mathrm{CDCl}_{3}\right)$

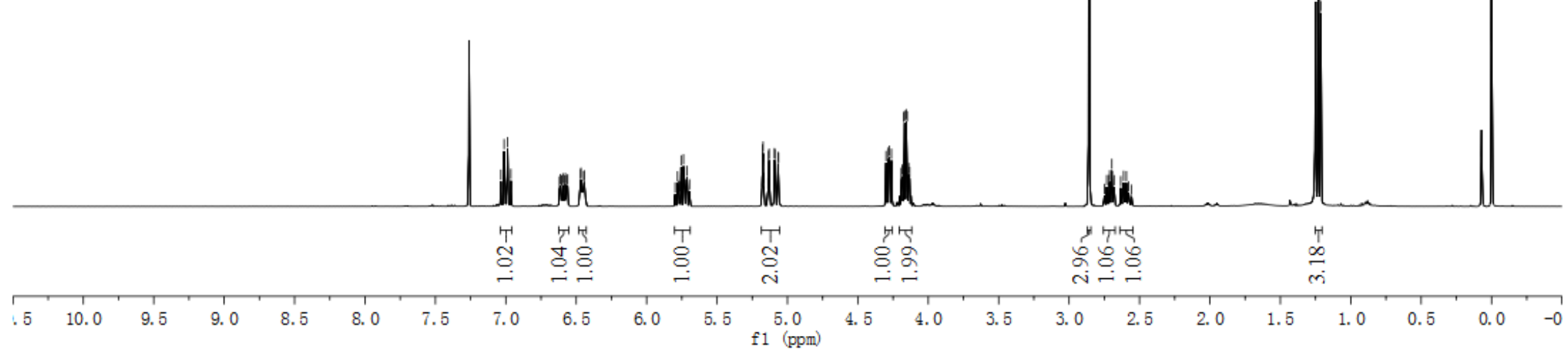

S-110 


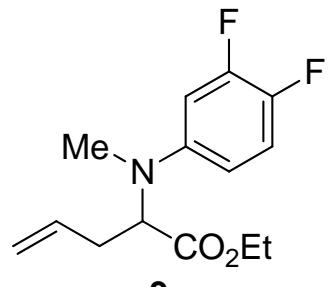

${ }^{13} \mathrm{C} \mathrm{NMR}\left(100 \mathrm{MHz}, \mathrm{CDCl}_{3}\right)$

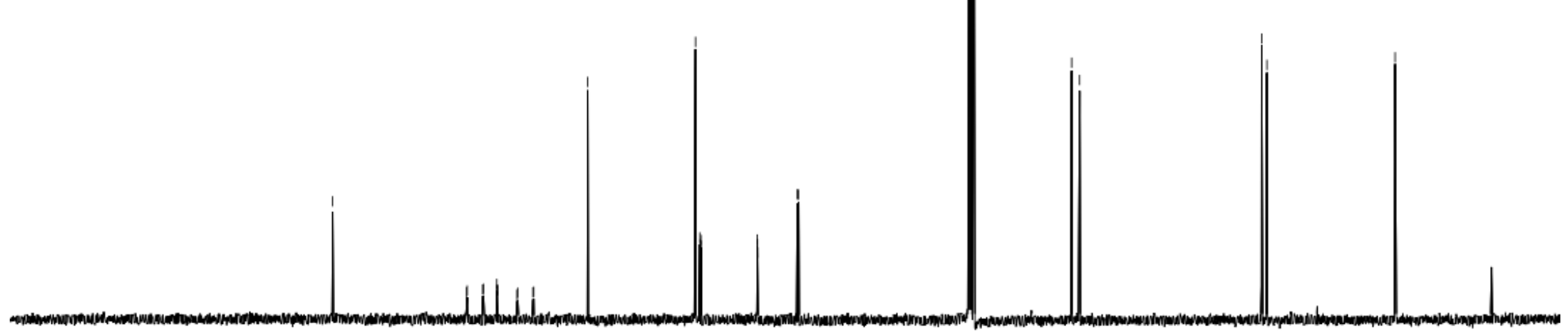

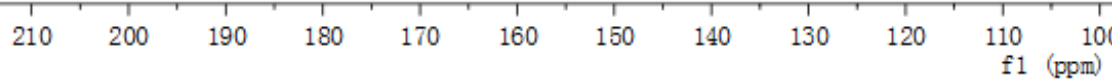

S-111 


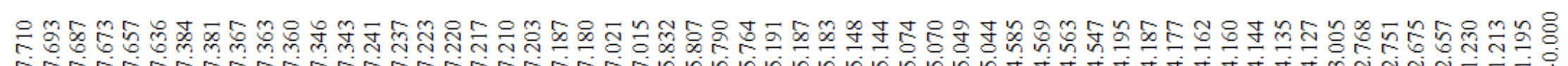

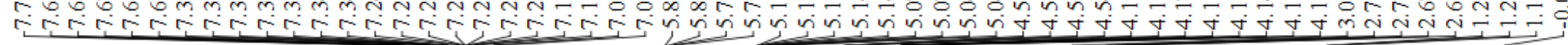

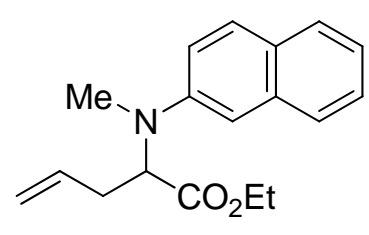

3y

${ }^{1} \mathrm{H} \mathrm{NMR}\left(400 \mathrm{MHz}, \mathrm{CDCl}_{3}\right)$

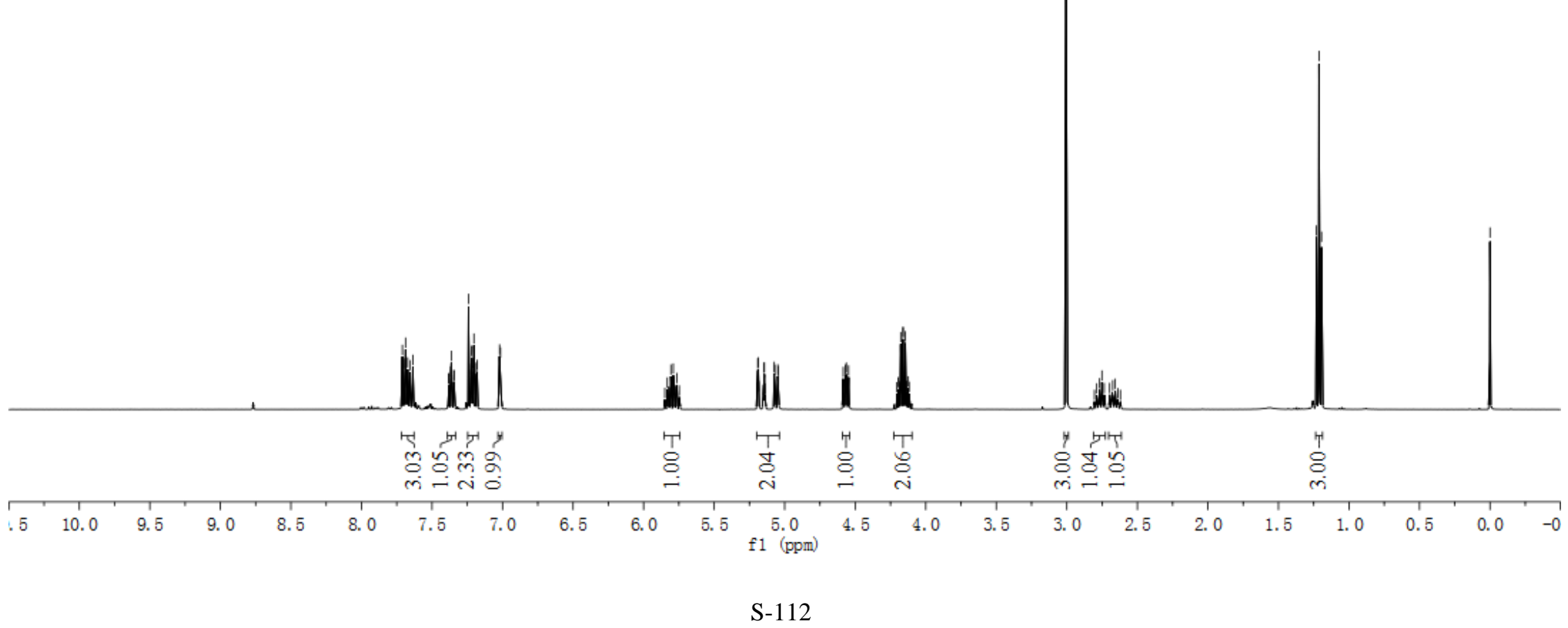



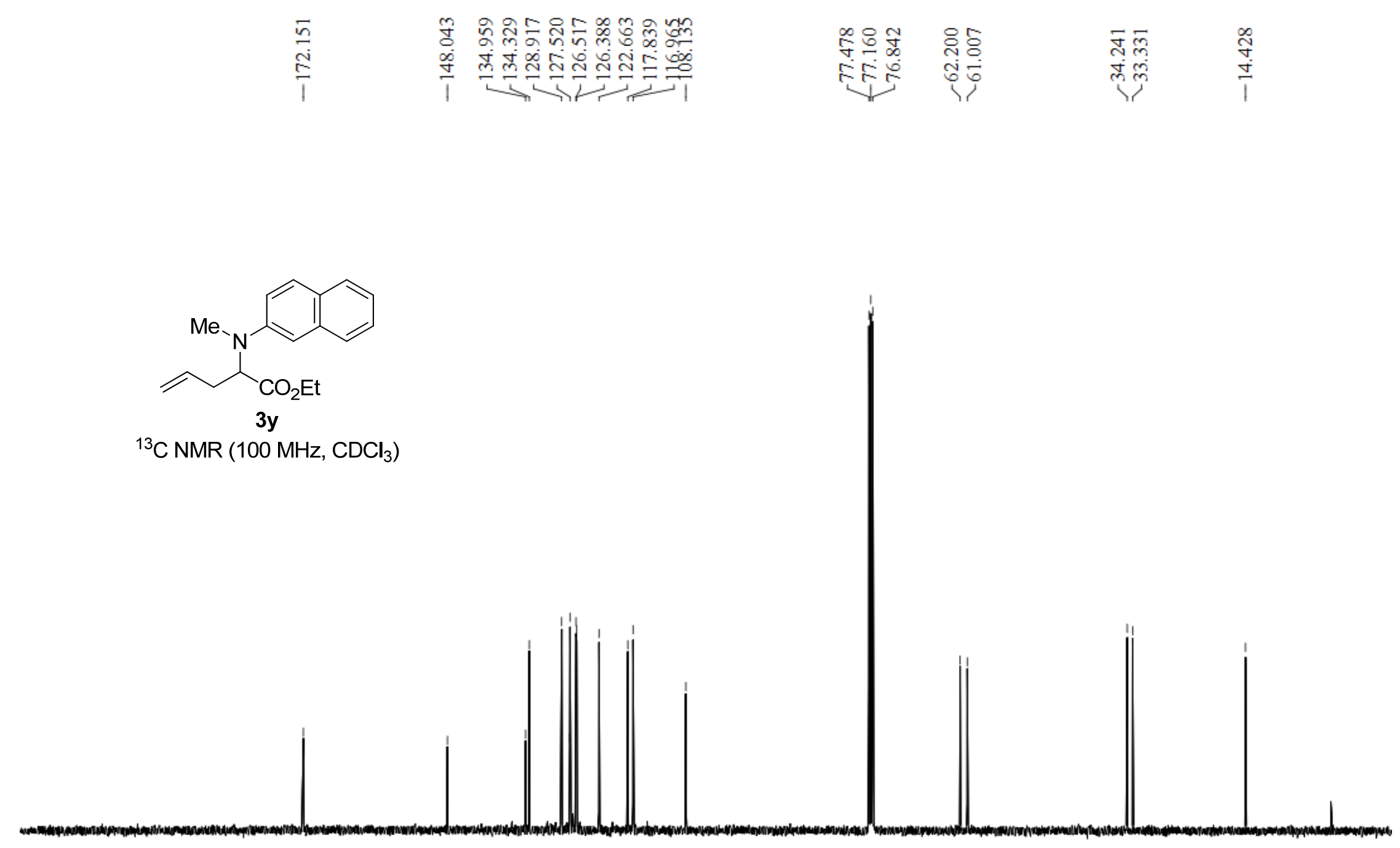

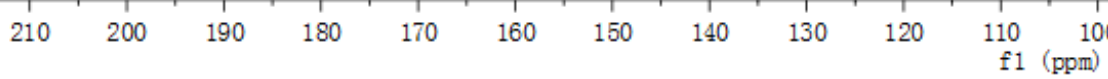

S-113 


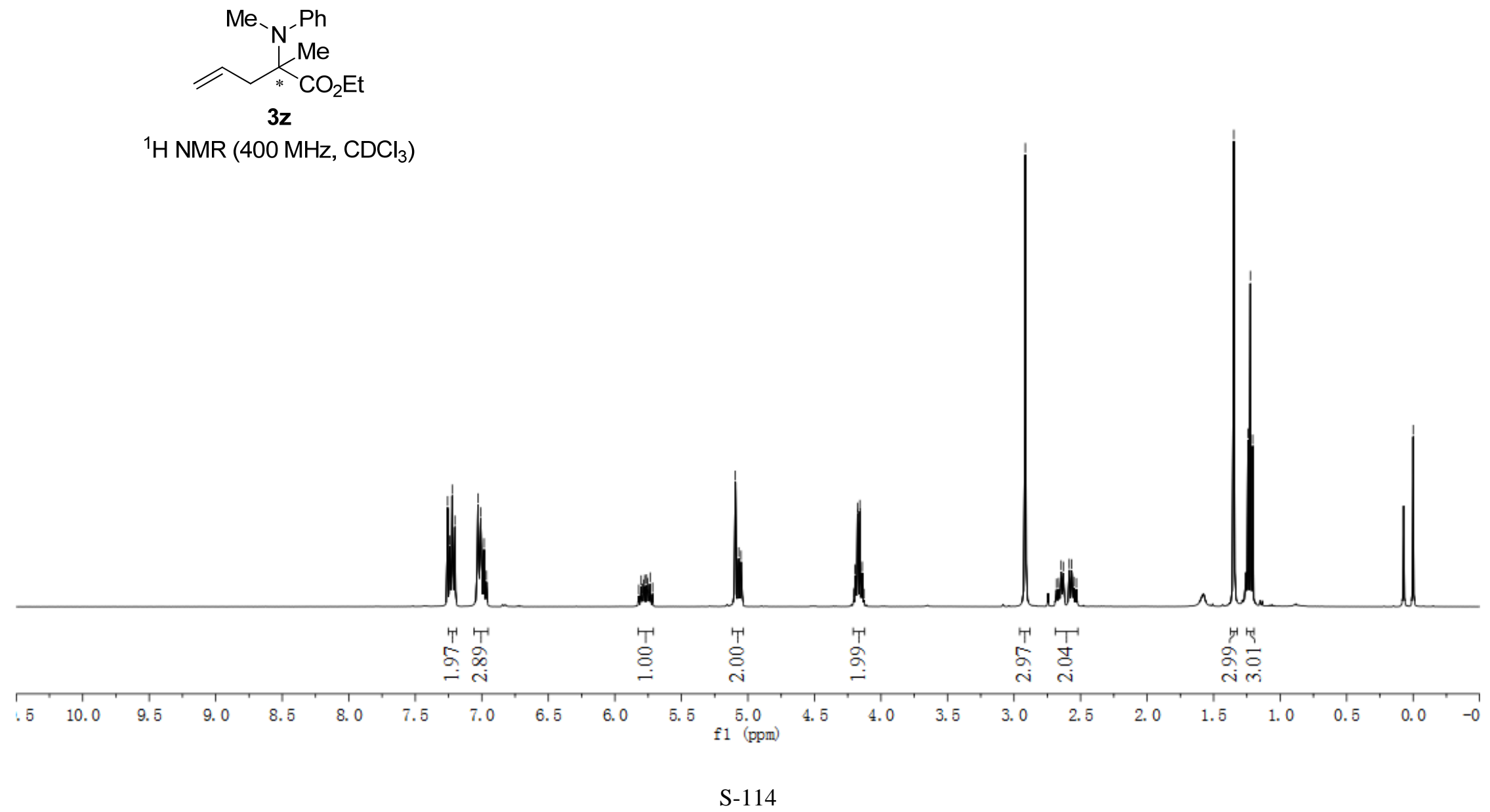




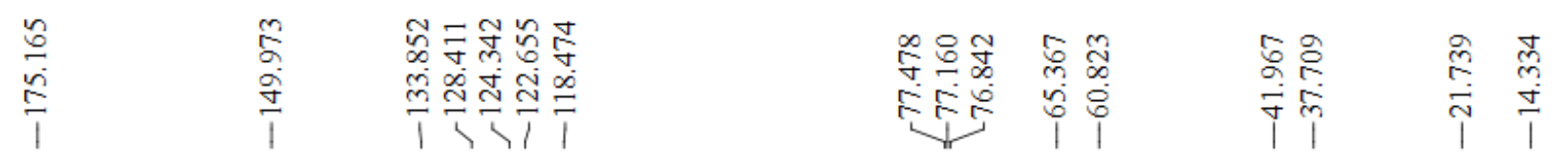

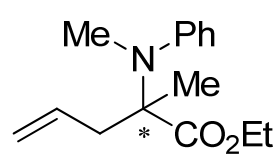

$3 z$

${ }^{13} \mathrm{C} \mathrm{NMR}\left(100 \mathrm{MHz}, \mathrm{CDCl}_{3}\right)$

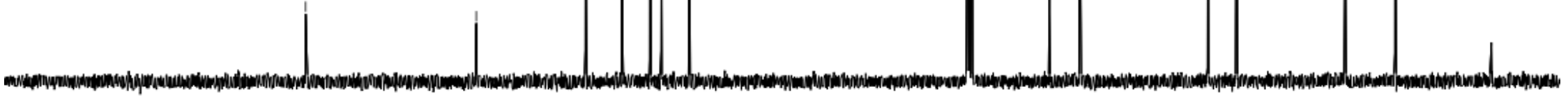

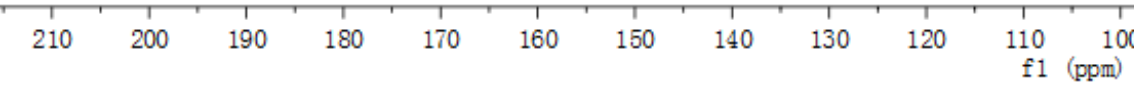

S-115 


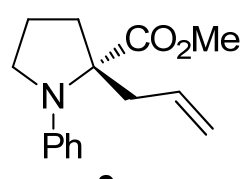

3aa

${ }^{1} \mathrm{H} \mathrm{NMR}\left(400 \mathrm{MHz}, \mathrm{CDCl}_{3}\right)$

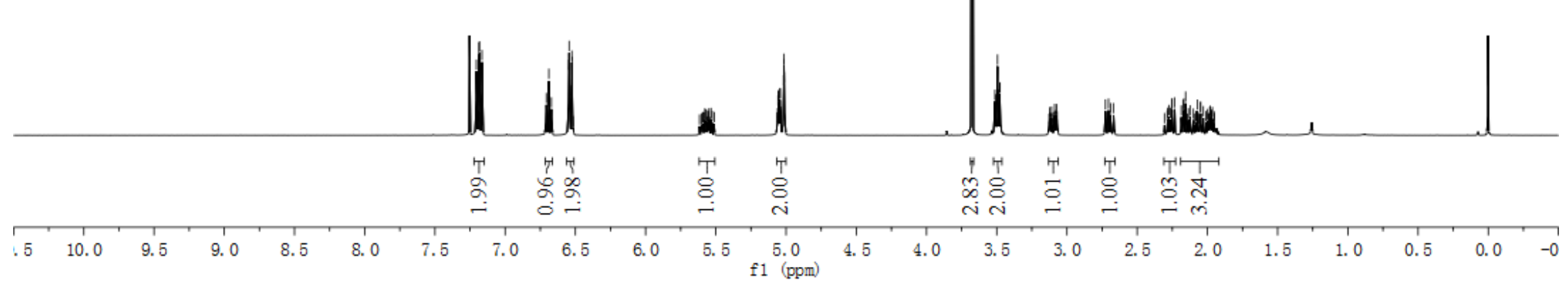

S-116 


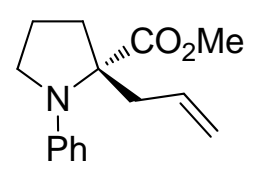

$$
\text { 3aa }
$$

${ }^{13} \mathrm{C}$ NMR $\left(100 \mathrm{MHz}, \mathrm{CDCl}_{3}\right)$

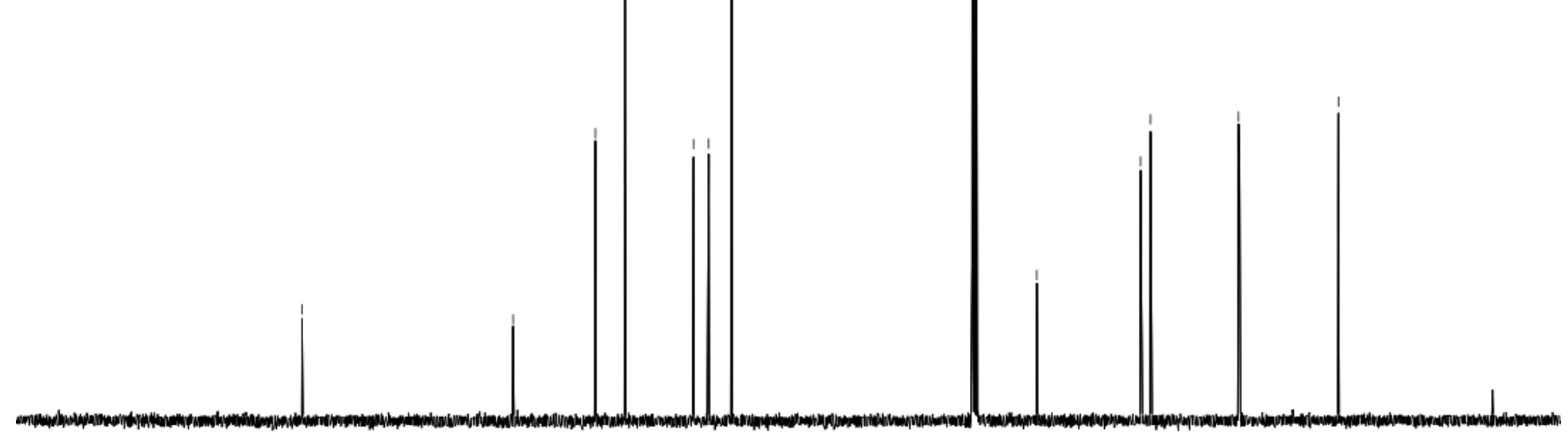

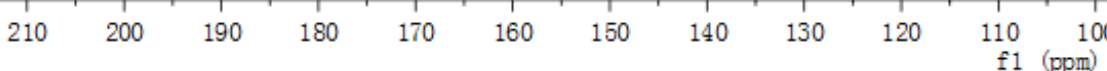




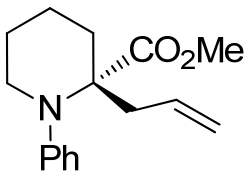

$3 a b$

${ }^{1} \mathrm{H} \mathrm{NMR}\left(400 \mathrm{MHz}, \mathrm{CDCl}_{3}\right)$

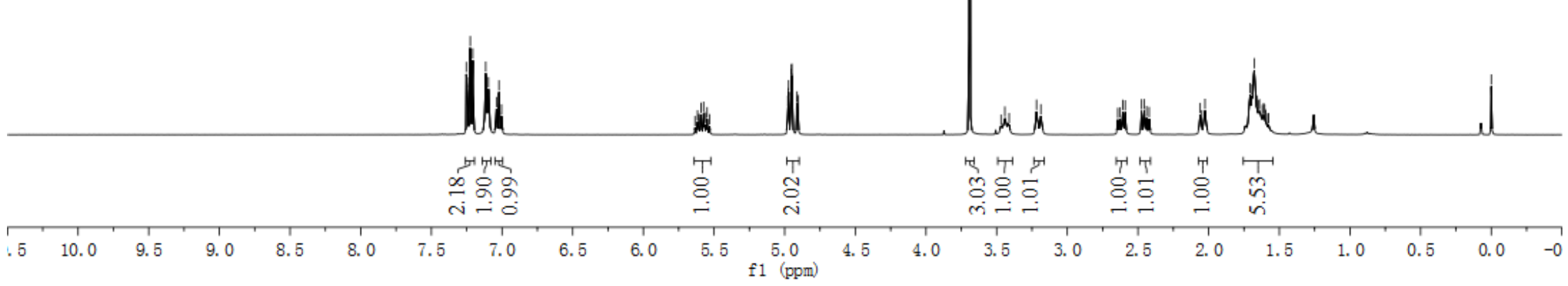

S-118 


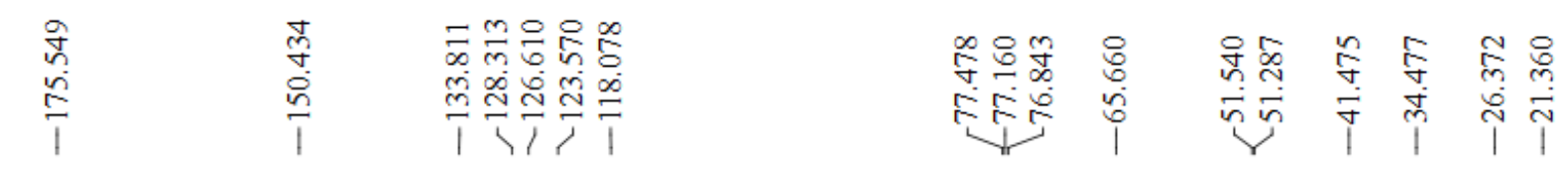

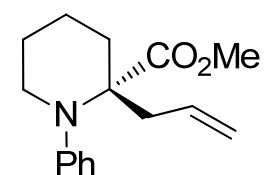

$3 a b$

${ }^{13} \mathrm{C} \mathrm{NMR}\left(100 \mathrm{MHz}, \mathrm{CDCl}_{3}\right)$

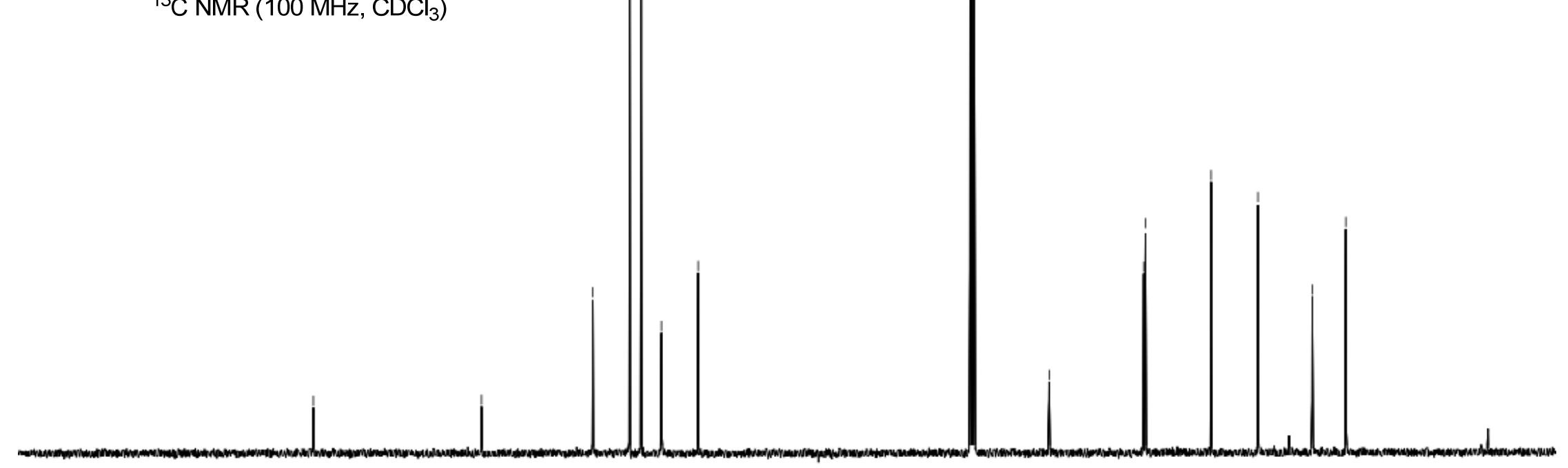

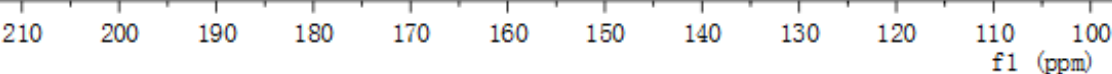

50

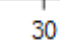

20

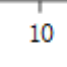

S-119 


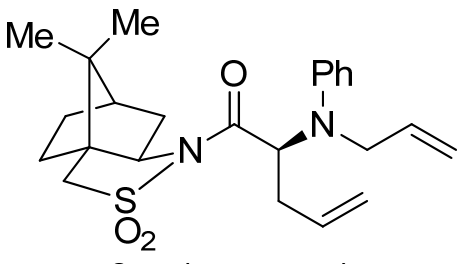

3ac (84: $16 \mathrm{dr})$

${ }^{1} \mathrm{H} \mathrm{NMR}\left(400 \mathrm{MHz}, \mathrm{CDCl}_{3}\right)$

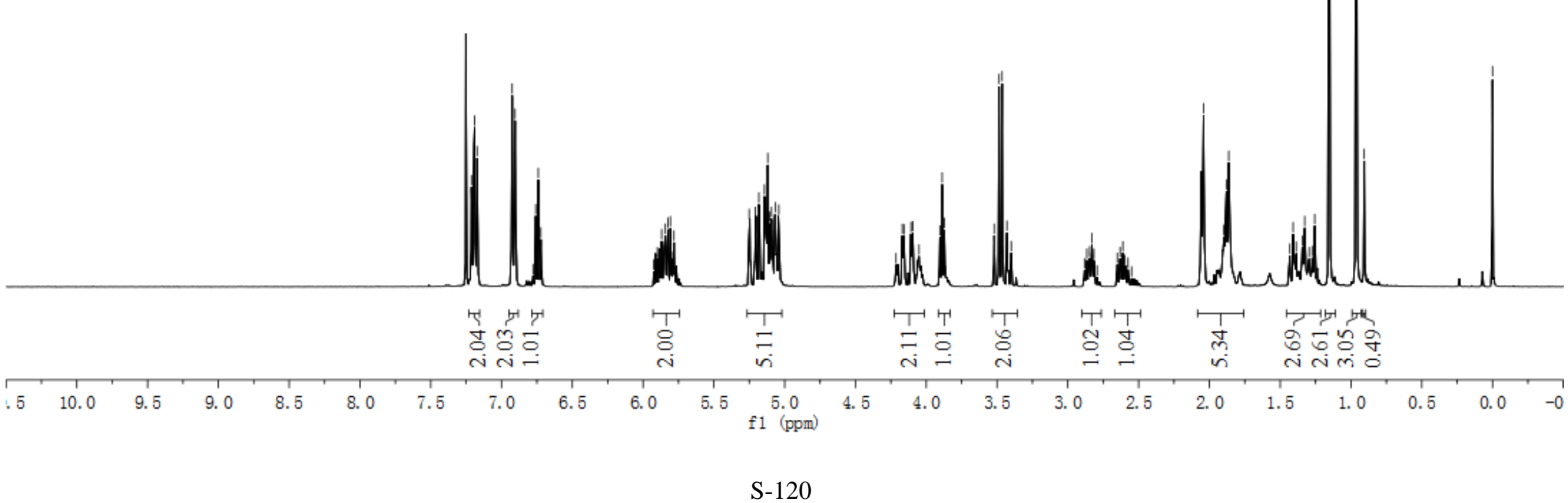




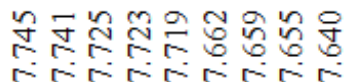

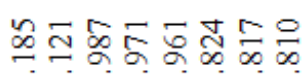

लMNNNलMN

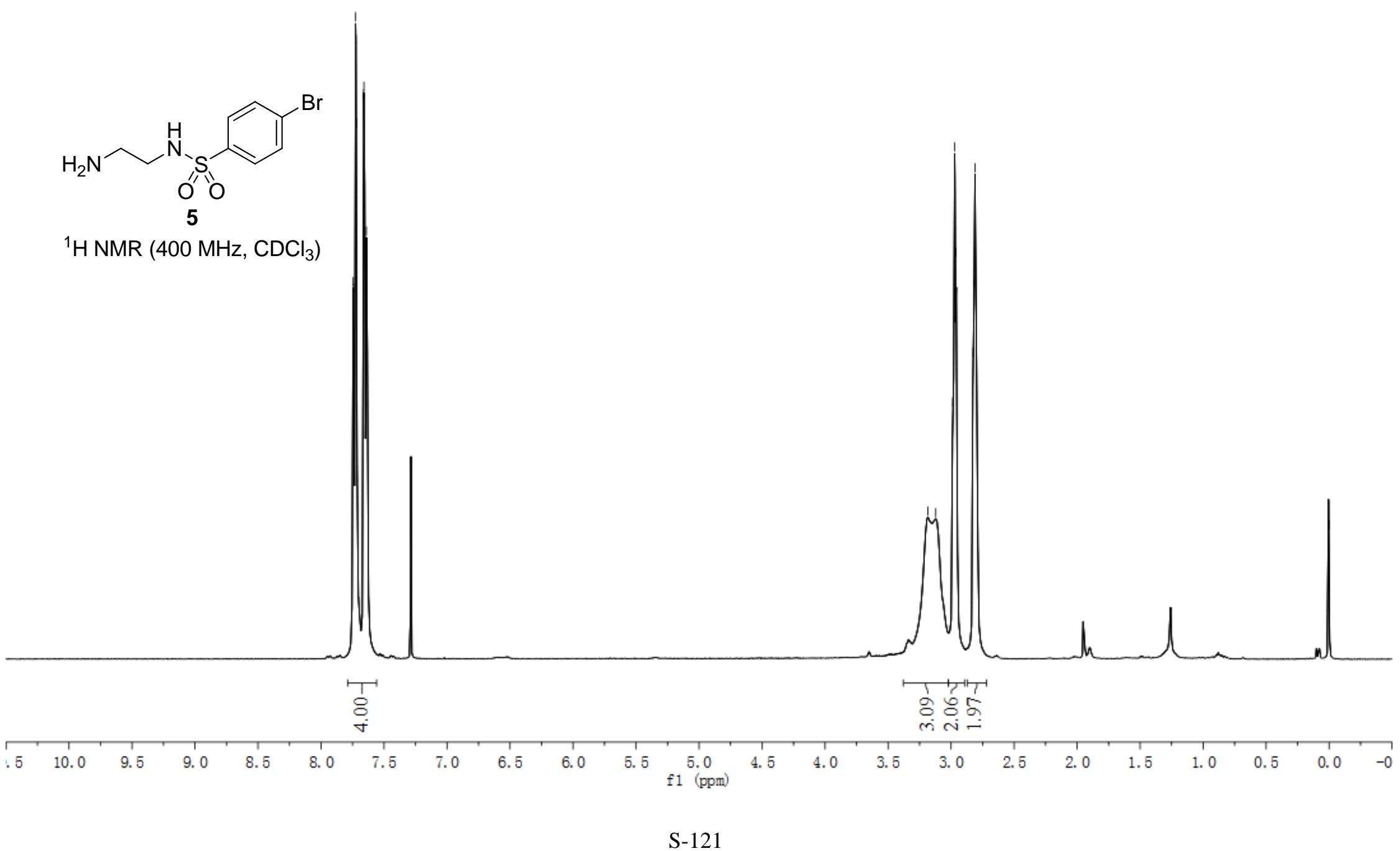




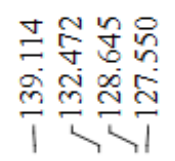

我赵

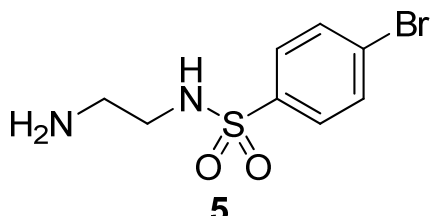

${ }^{13} \mathrm{C}$ NMR $\left(100 \mathrm{MHz}, \mathrm{CDCl}_{3}\right)$
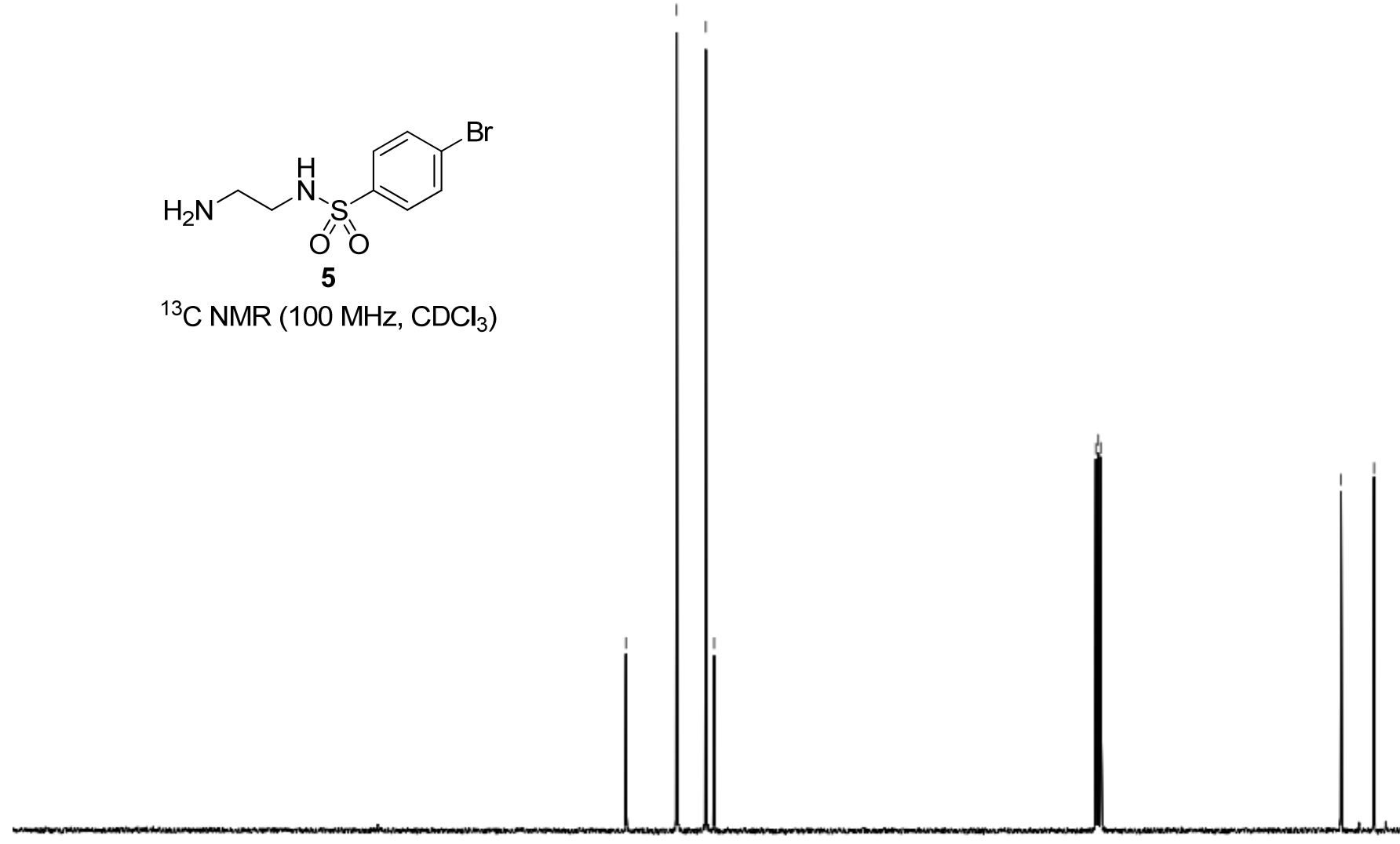

$\begin{array}{lllllll}210 & 200 & 190 & 180 & 170 & 160 & 160\end{array}$

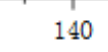

130

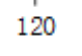

$\begin{array}{cc}110 & 100 \\ \mathrm{f} 1 & (\mathrm{ppm})\end{array}$

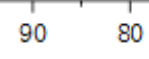

70

60
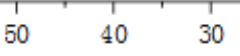


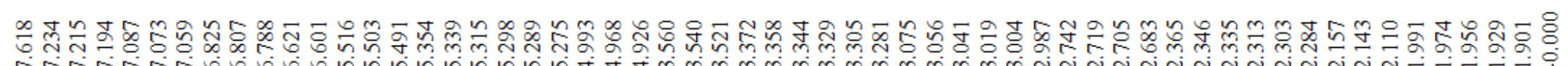

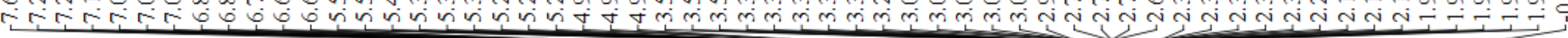
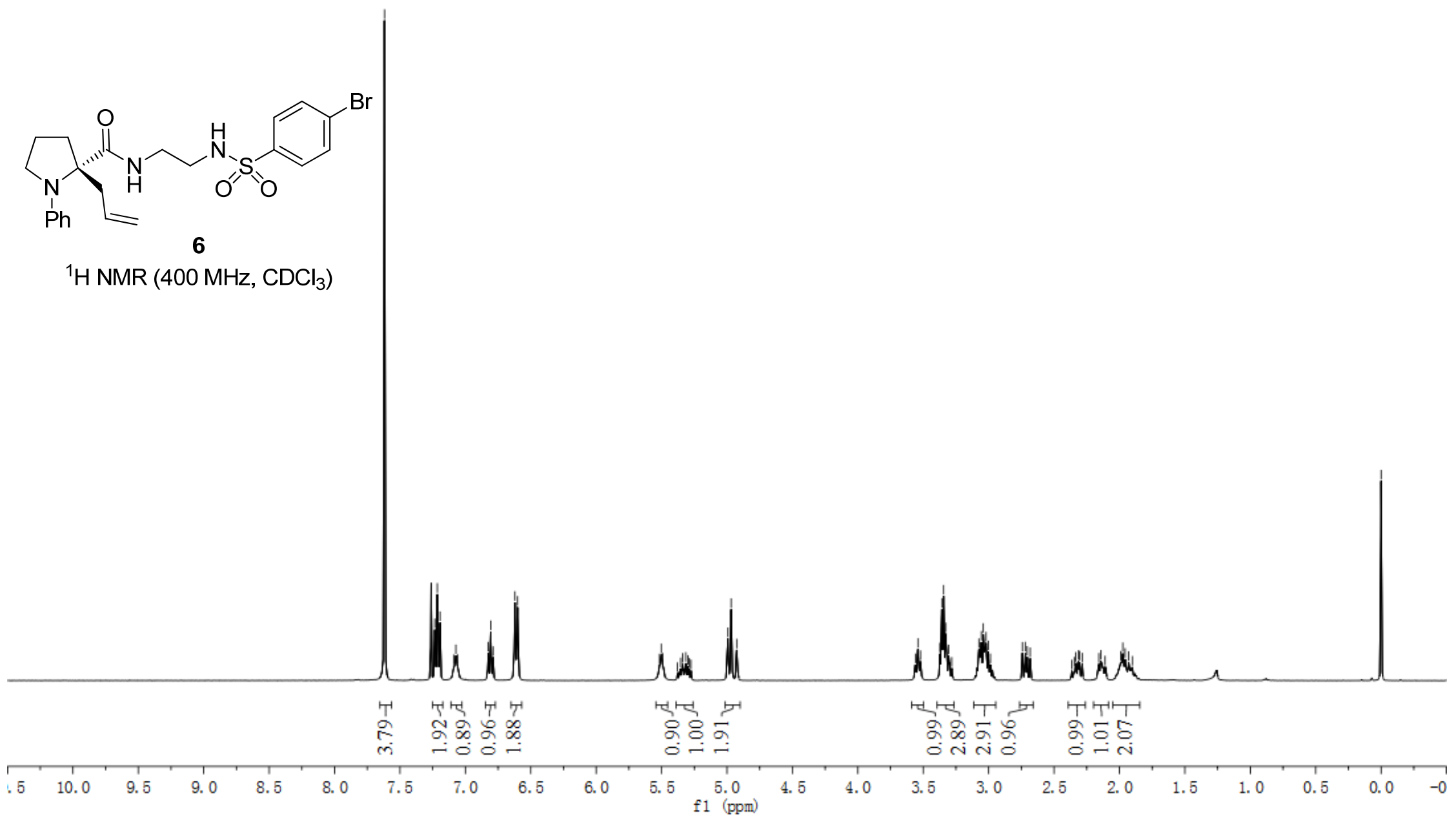

S-123 

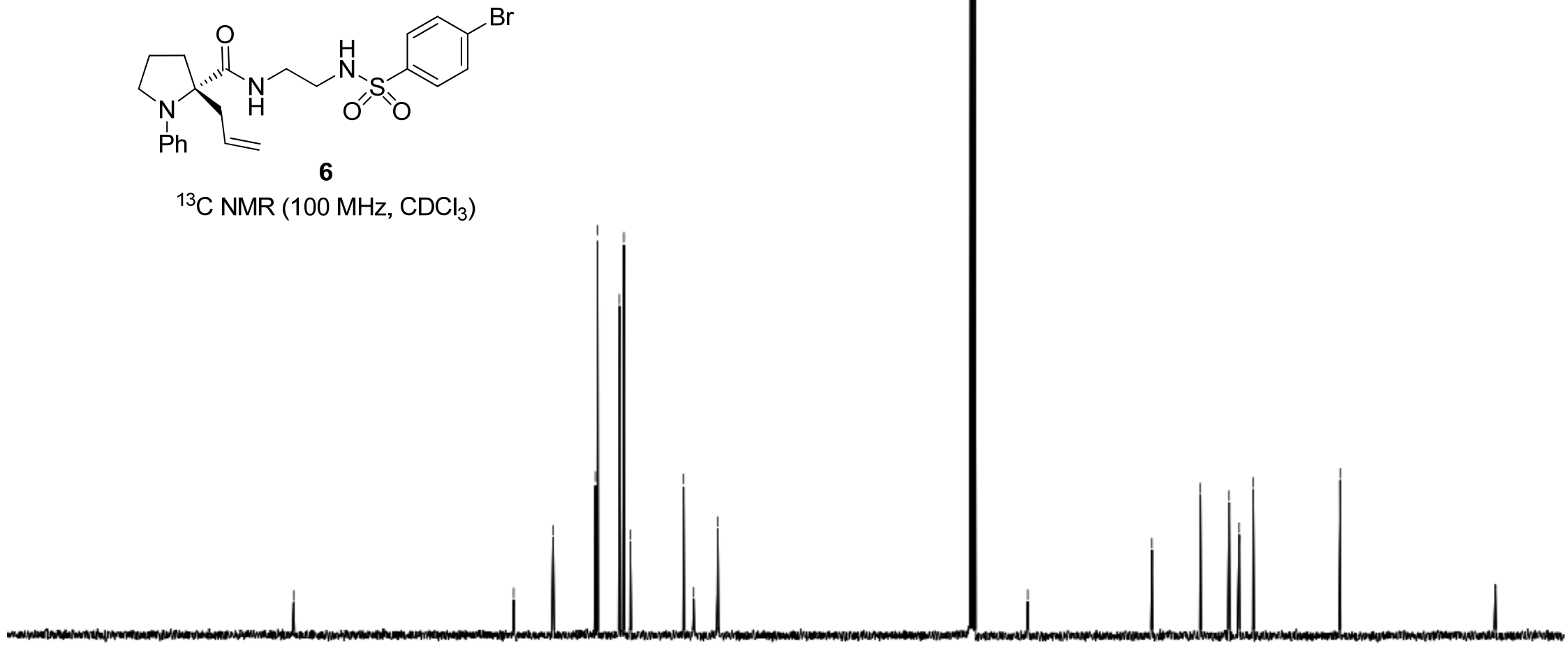


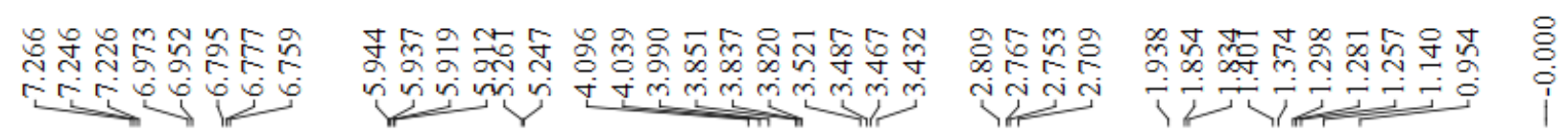

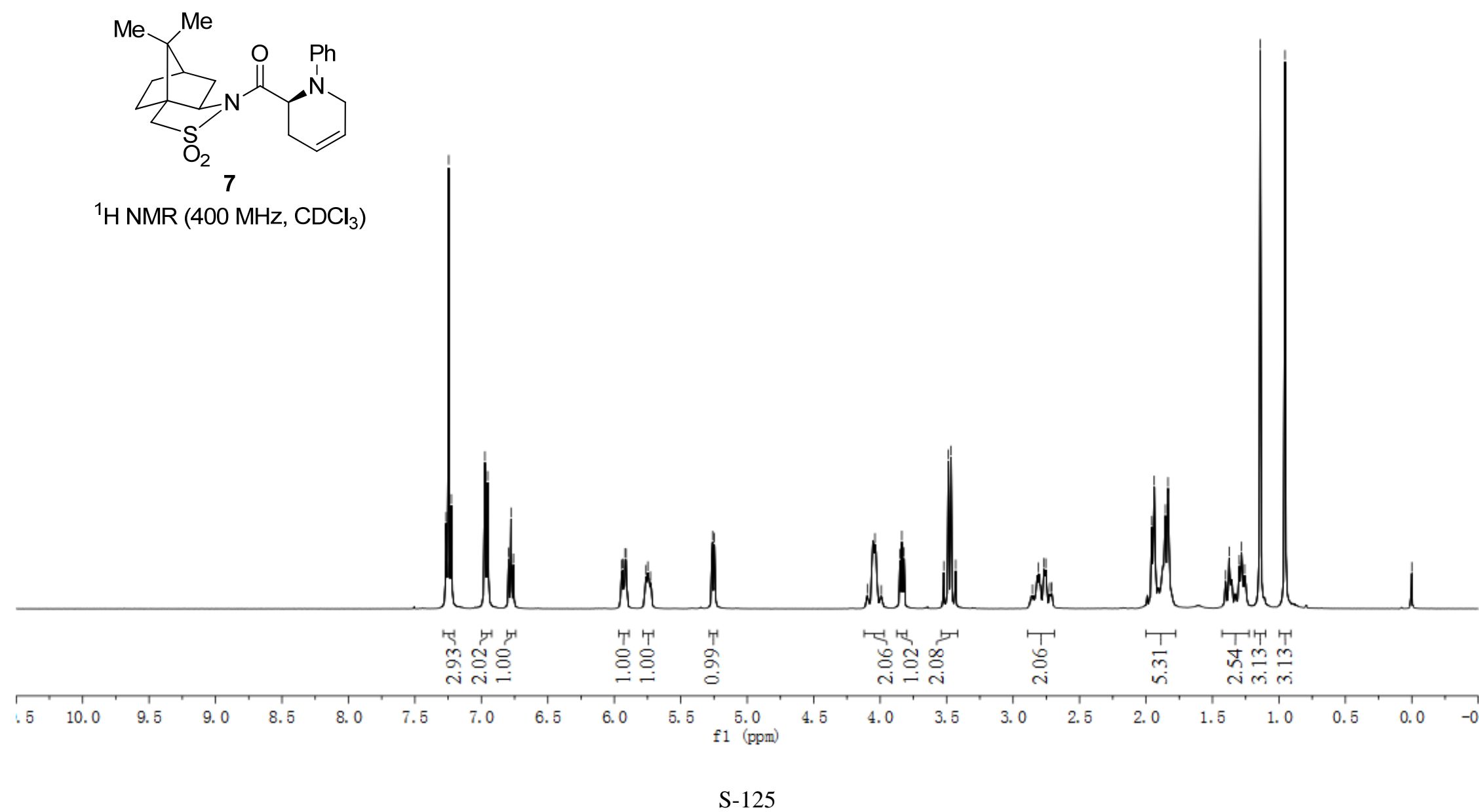



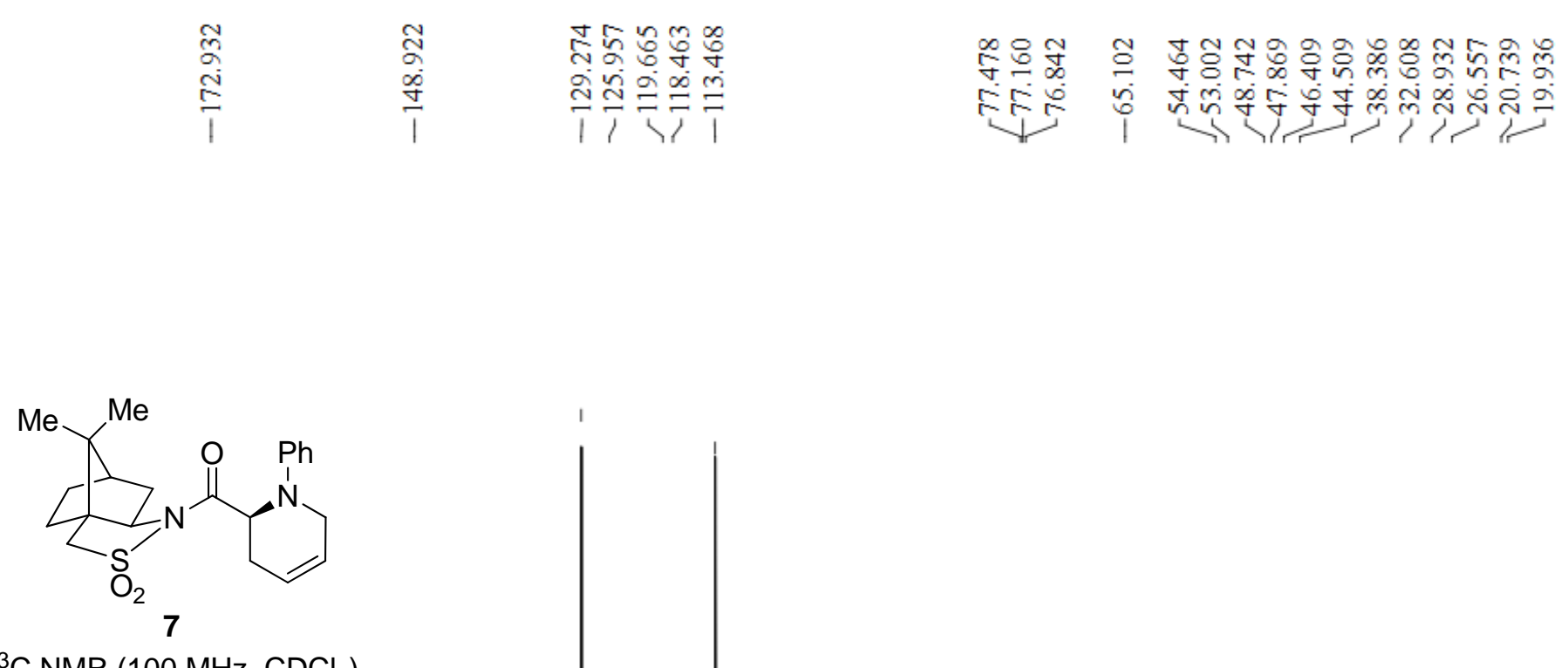

${ }^{13} \mathrm{C}$ NMR $\left(100 \mathrm{MHz}, \mathrm{CDCl}_{3}\right)$

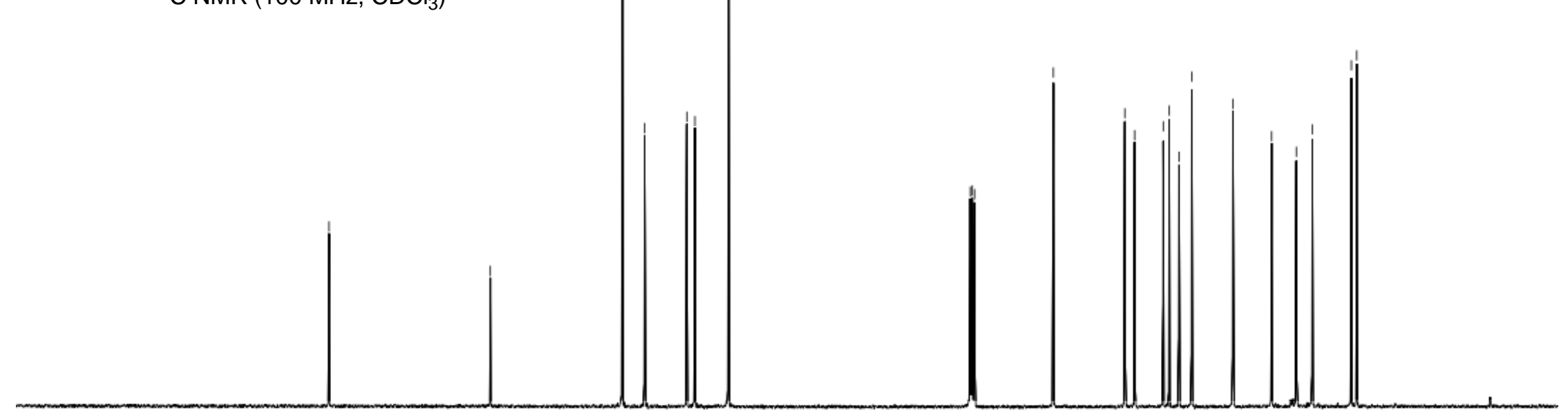




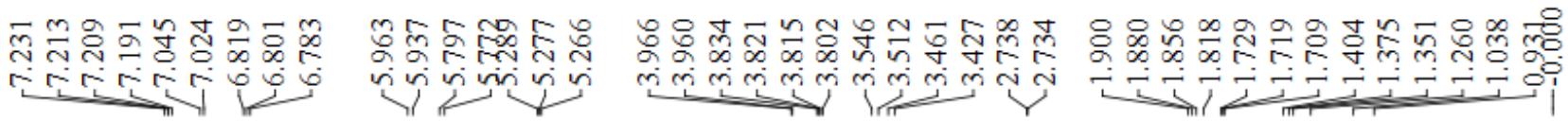

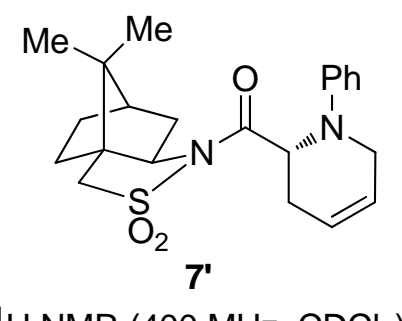

${ }^{1} \mathrm{H}$ NMR $\left(400 \mathrm{MHz}, \mathrm{CDCl}_{3}\right)$

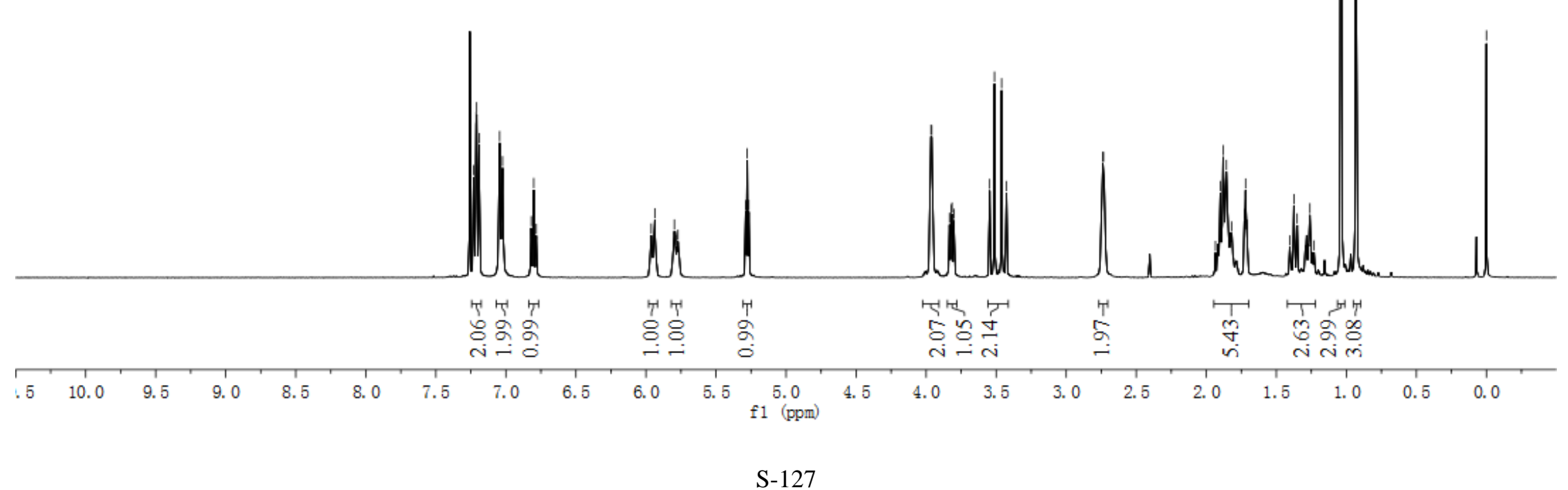



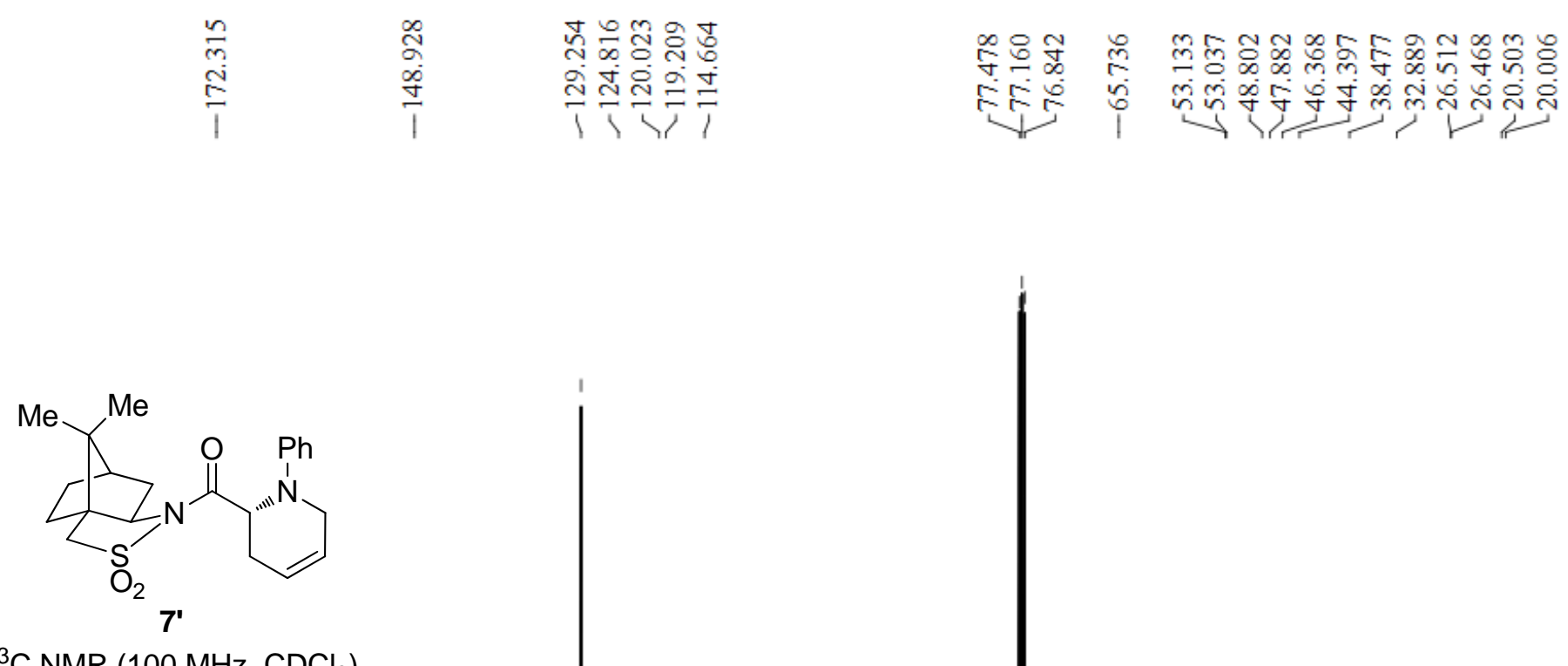

${ }^{13} \mathrm{C}$ NMR $\left(100 \mathrm{MHz}, \mathrm{CDCl}_{3}\right)$

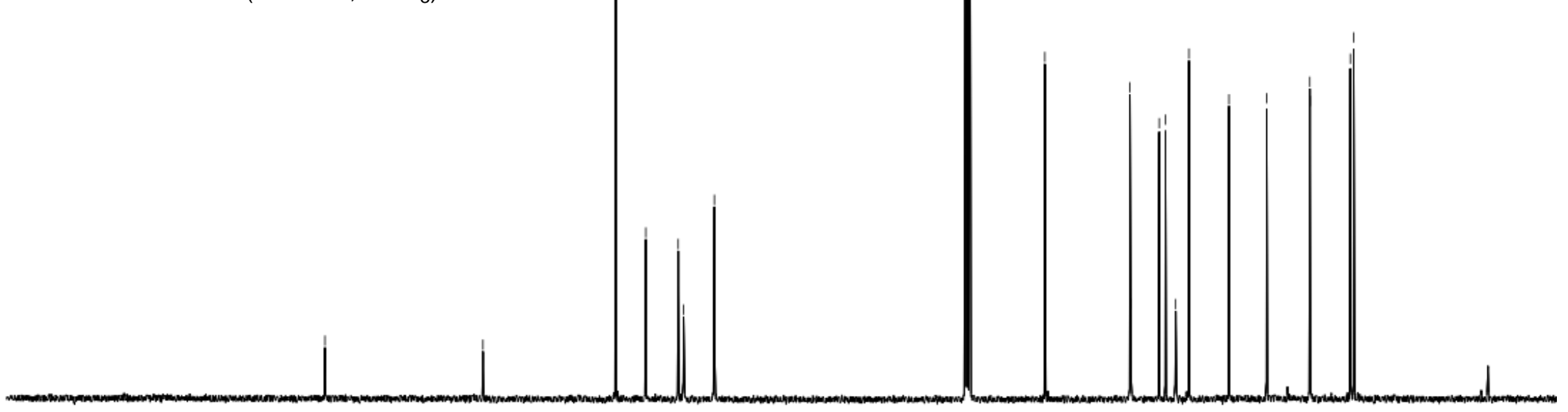

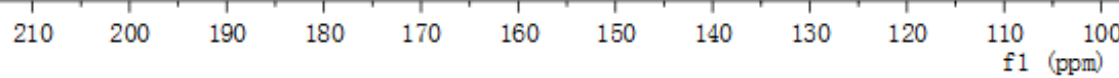

S-128 


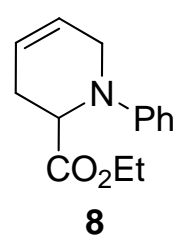

${ }^{1} \mathrm{H} \mathrm{NMR}\left(400 \mathrm{MHz}, \mathrm{CDCl}_{3}\right)$

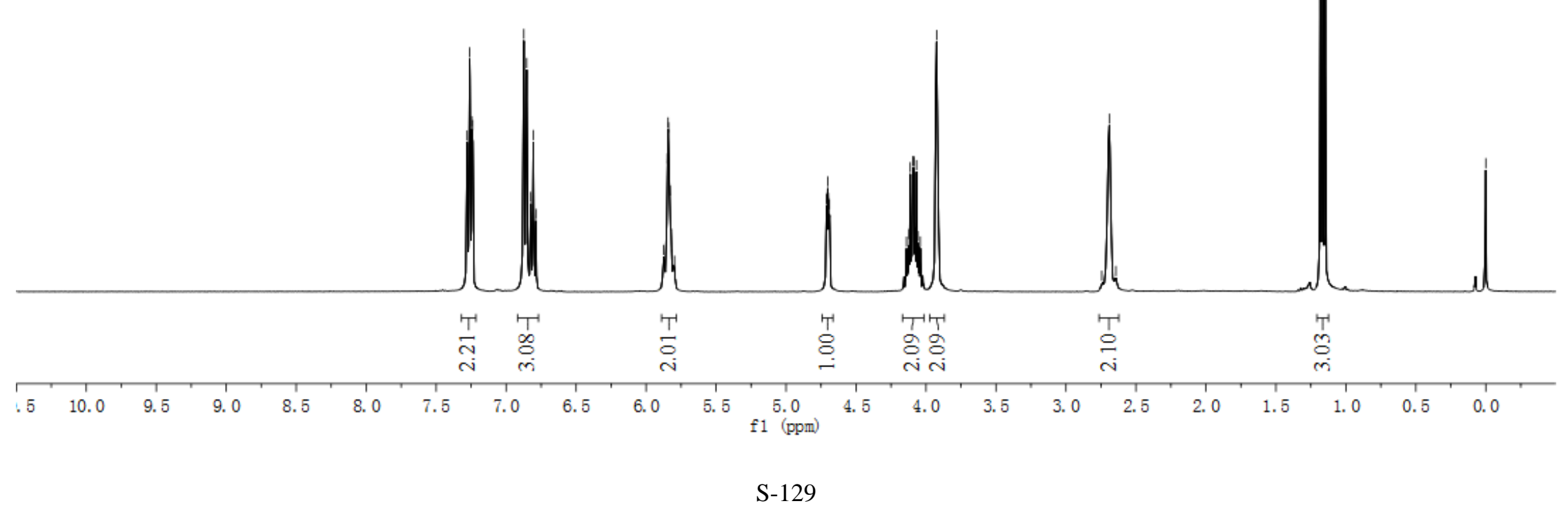




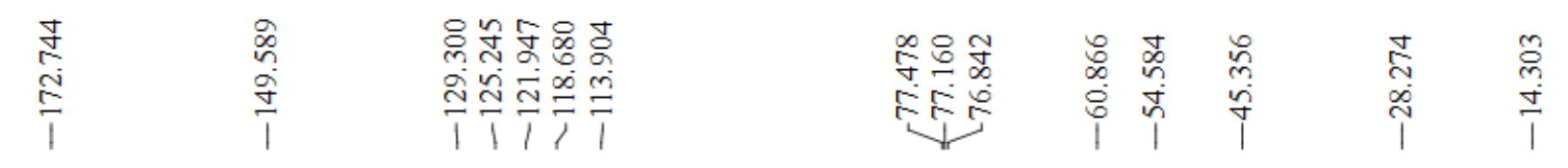

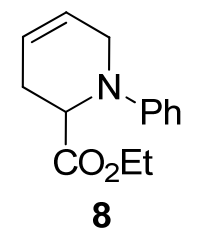

${ }^{13} \mathrm{C}$ NMR $\left(100 \mathrm{MHz}, \mathrm{CDCl}_{3}\right)$

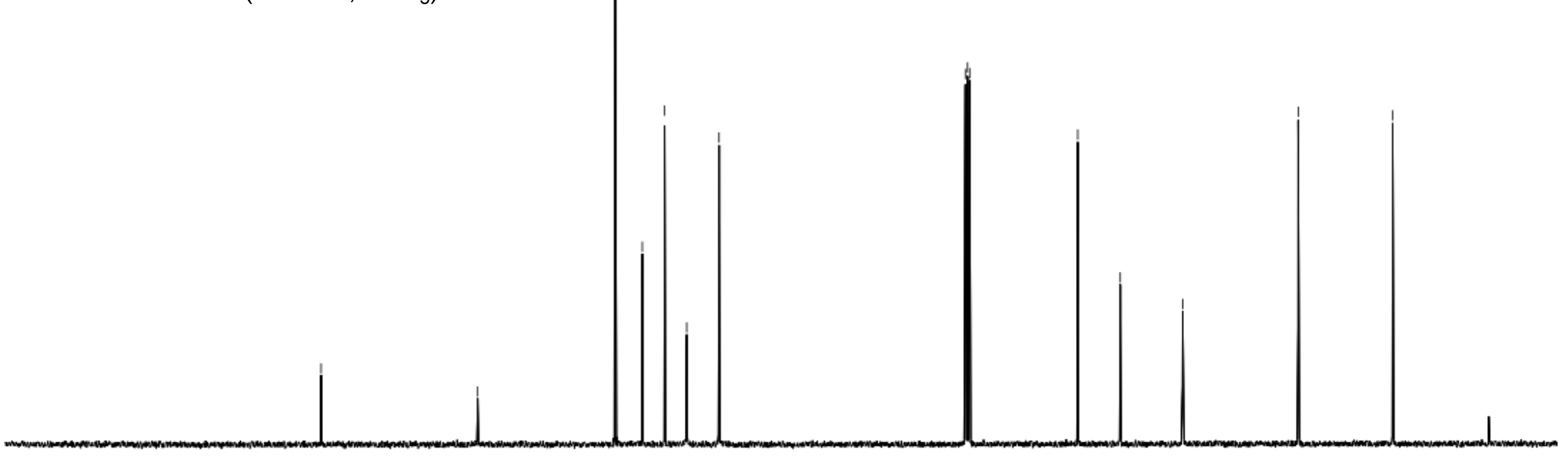

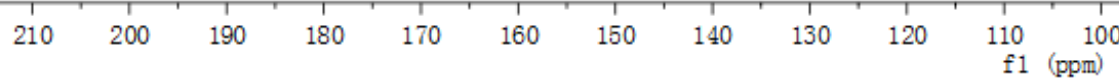

S-130 


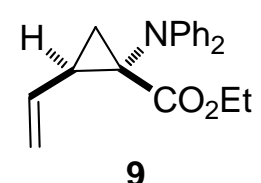

${ }^{1} \mathrm{H}$ NMR $\left(400 \mathrm{MHz}, \mathrm{CDCl}_{3}\right)$

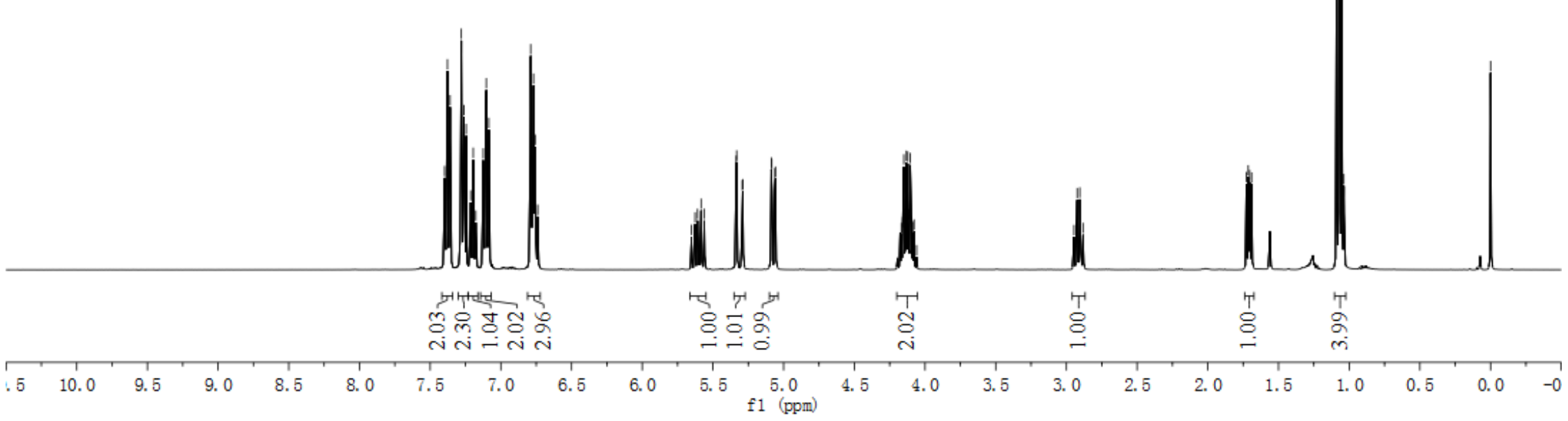




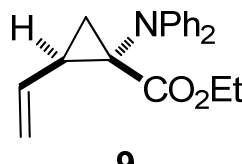

9

${ }^{13} \mathrm{C} \mathrm{NMR}\left(100 \mathrm{MHz}, \mathrm{CDCl}_{3}\right)$
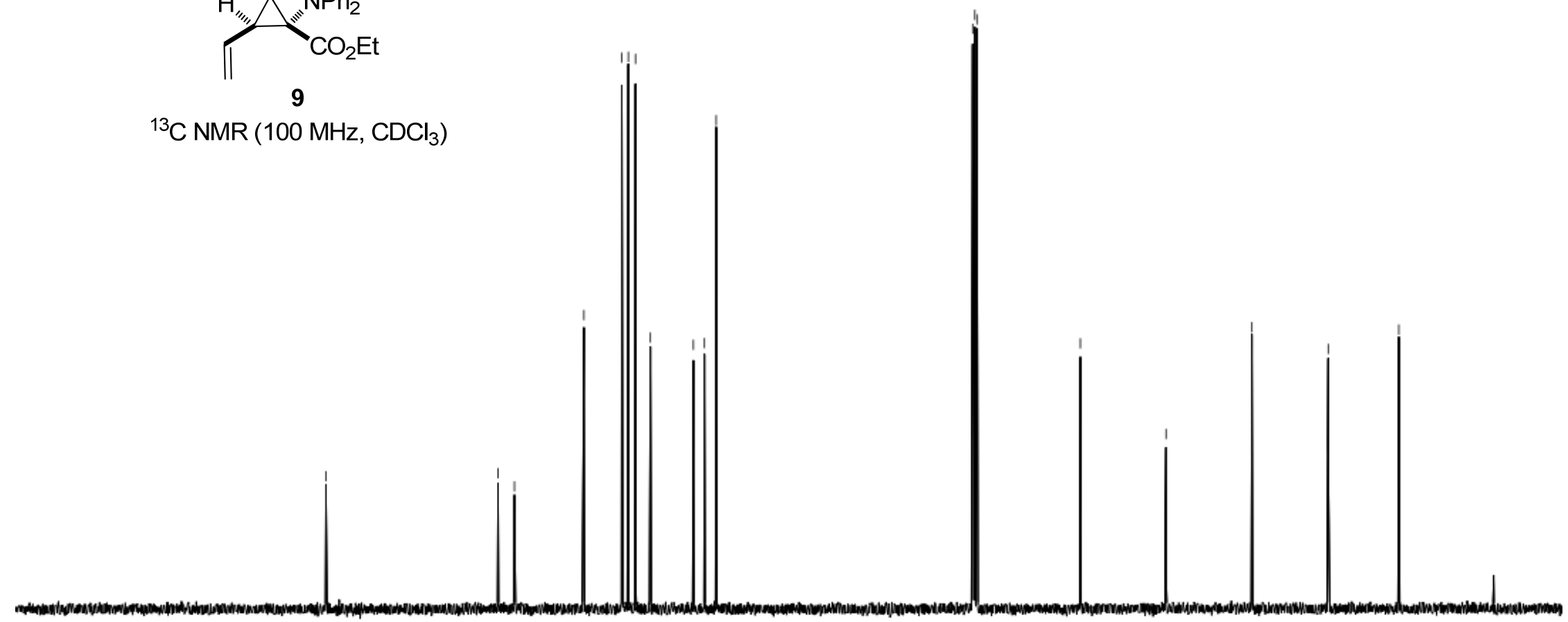

$210 \quad 200 \quad 190$

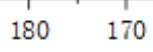

160

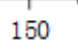

$140 \quad 130$

$120 \quad 110 \quad 100$

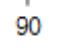

80

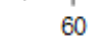

50
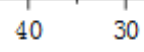


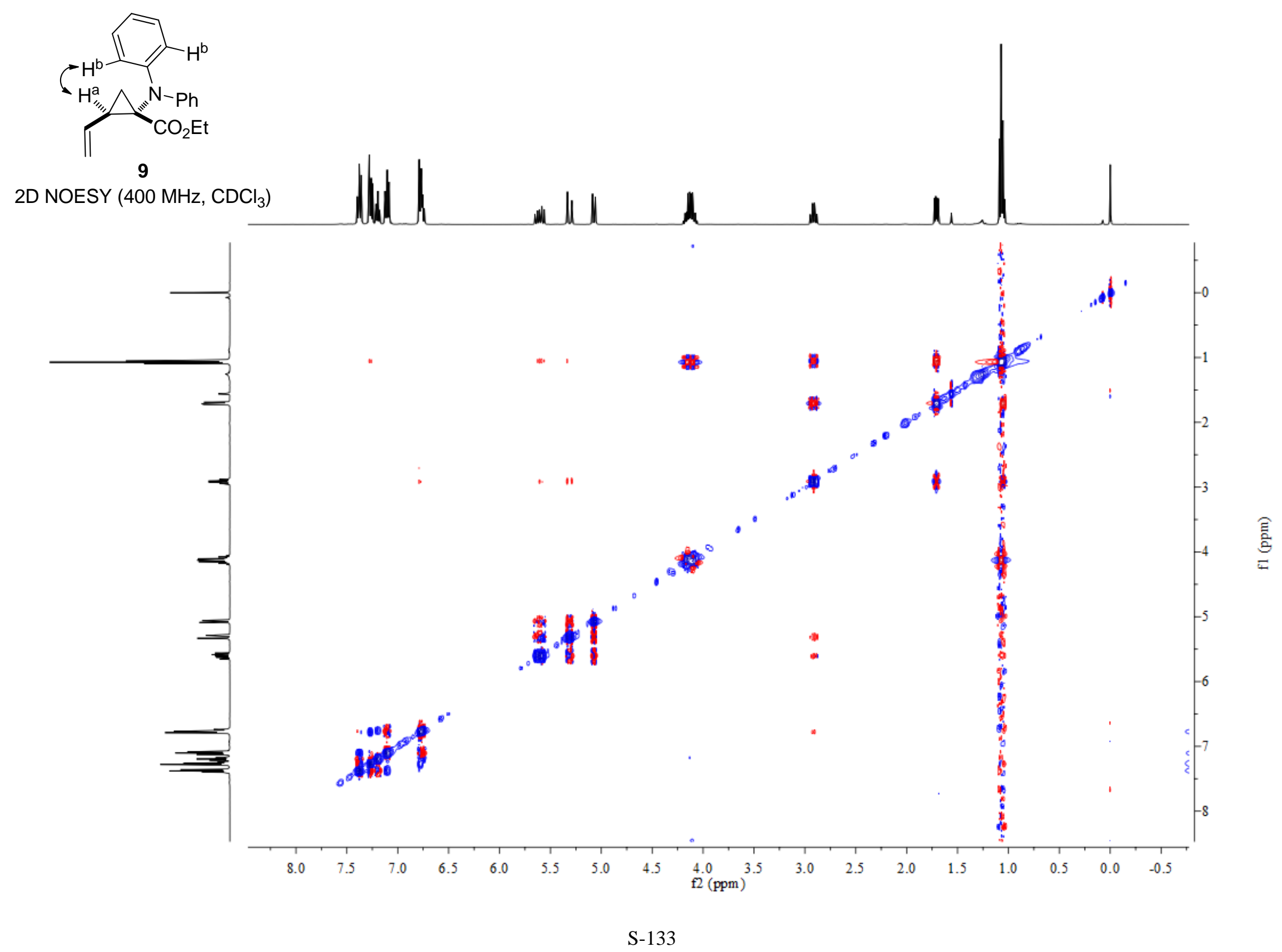




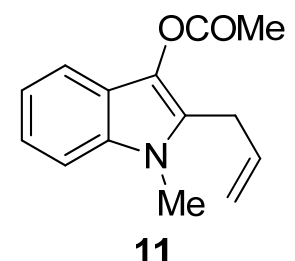

${ }^{1} \mathrm{H} \mathrm{NMR}\left(400 \mathrm{MHz}, \mathrm{CDCl}_{3}\right)$

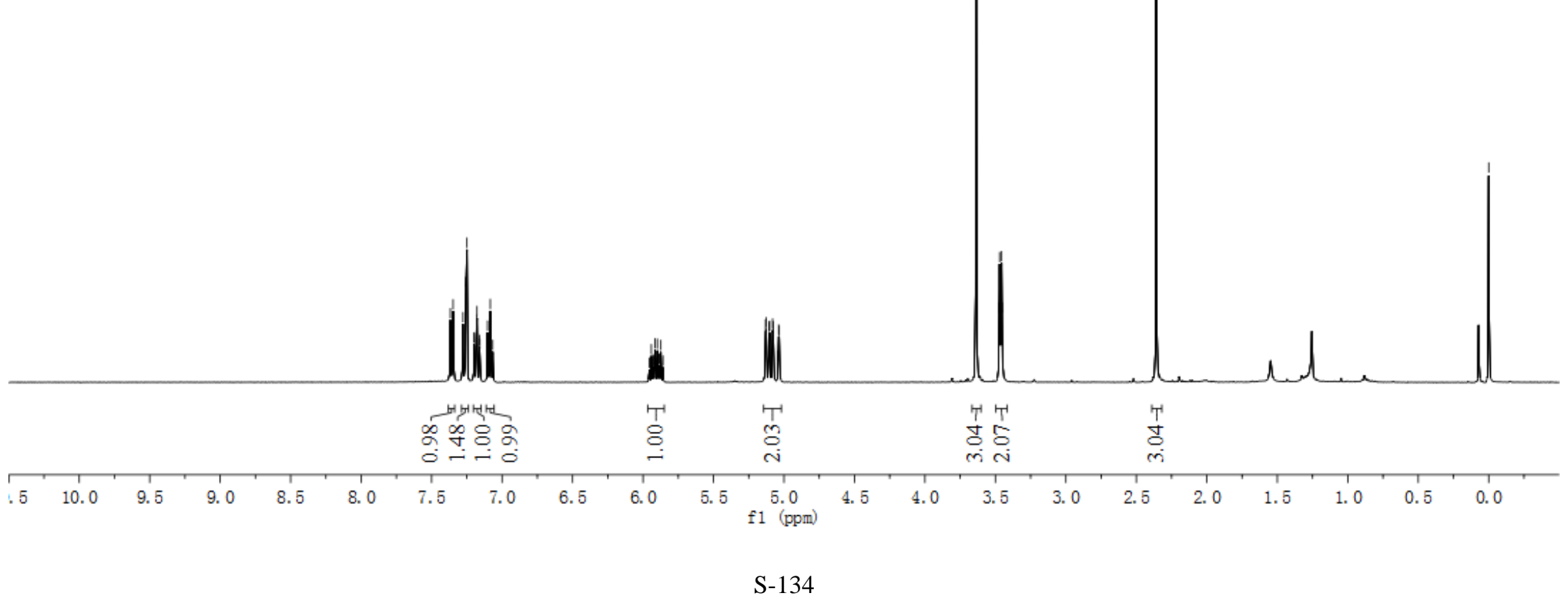



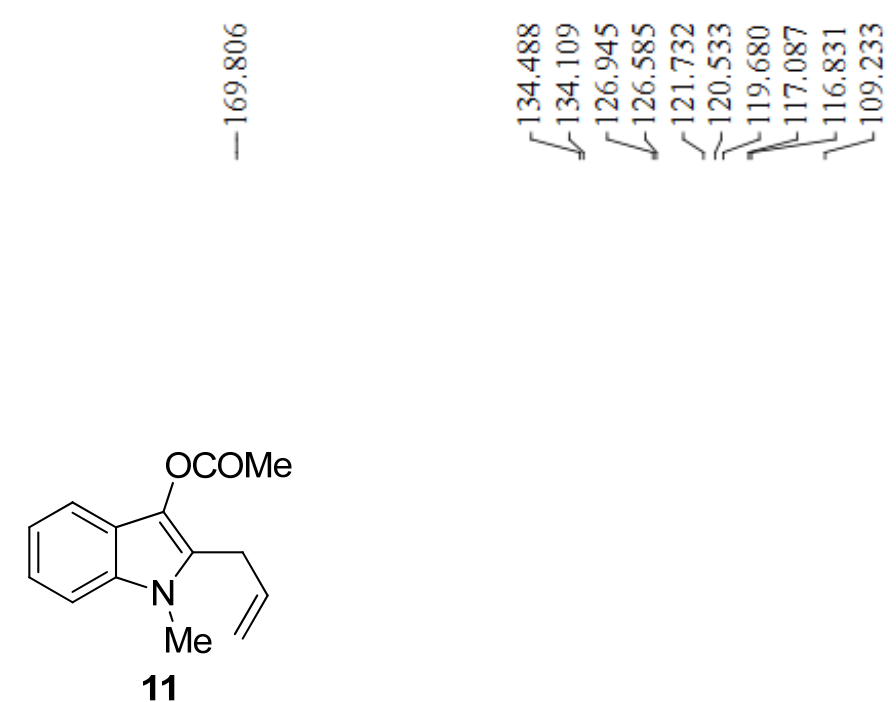

$\stackrel{\infty}{\forall} \underset{\sim}{\infty} \stackrel{m}{\infty}$

के

iิं i্ं

${ }^{13} \mathrm{C} \mathrm{NMR}\left(100 \mathrm{MHz}, \mathrm{CDCl}_{3}\right)$

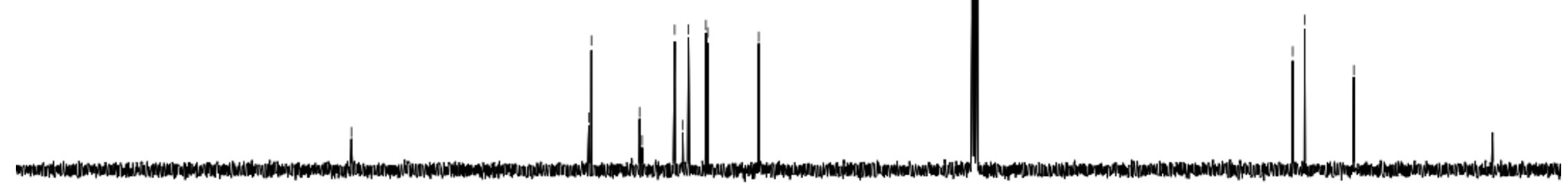

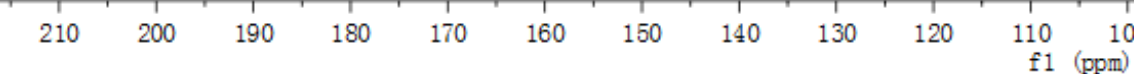

S-135 

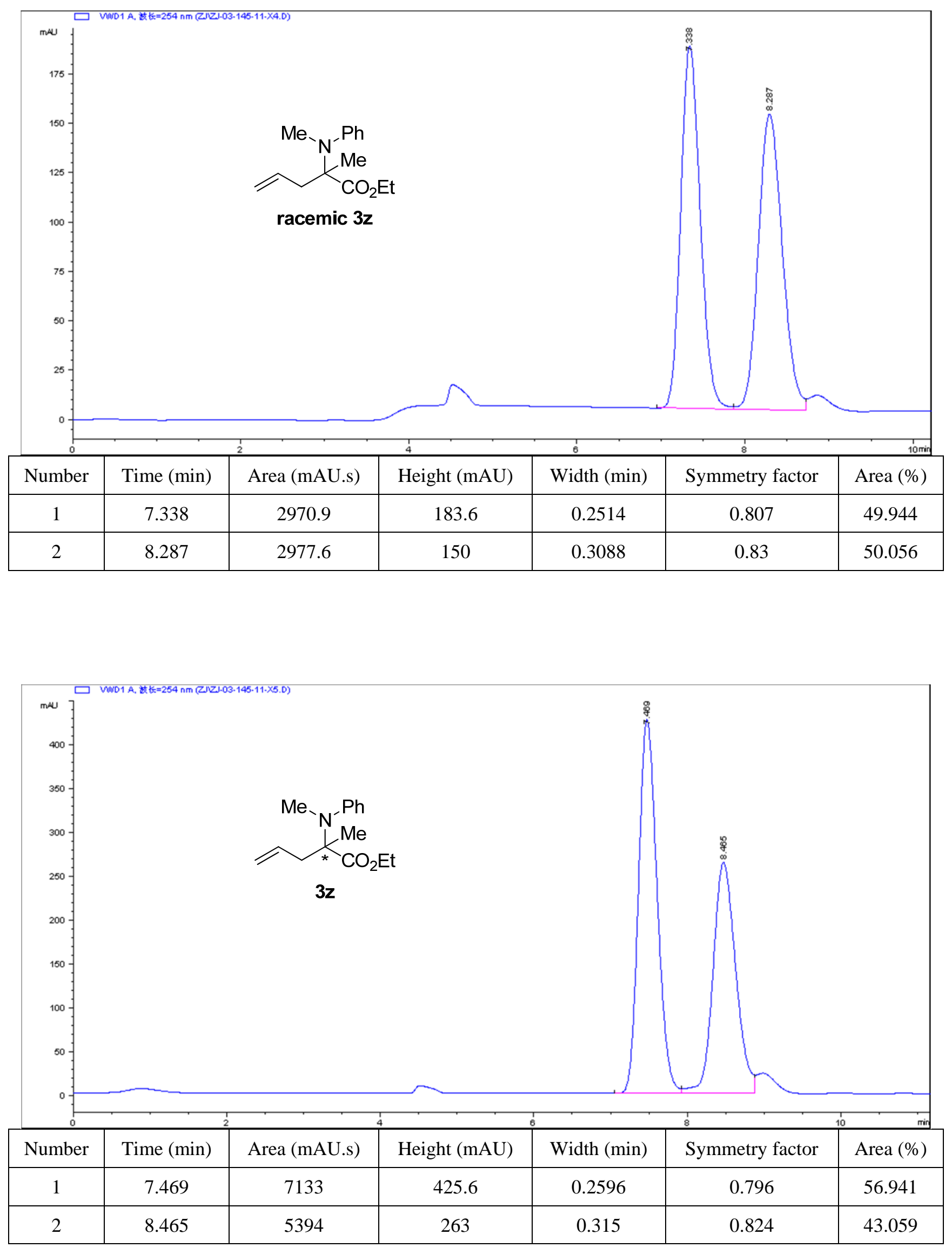

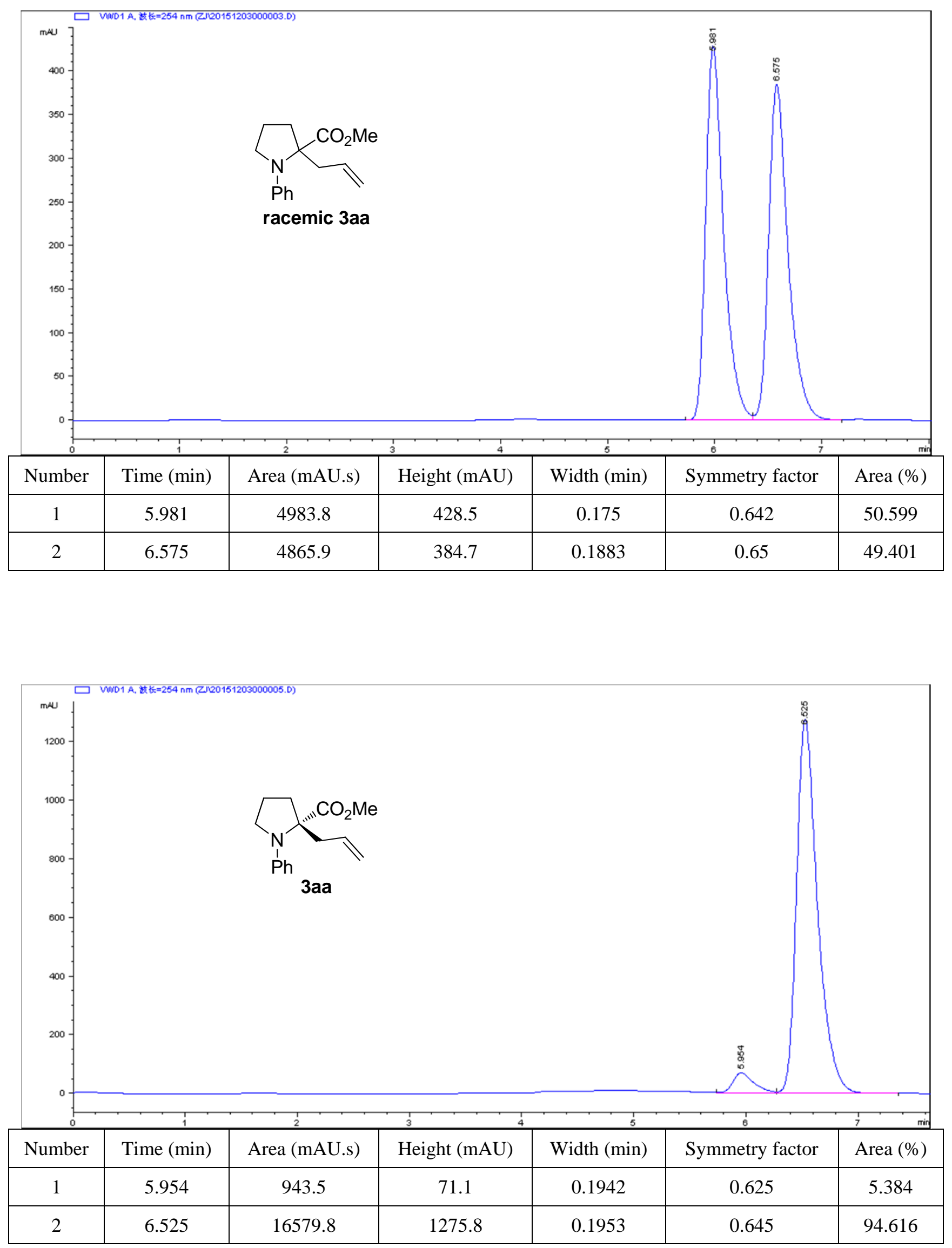

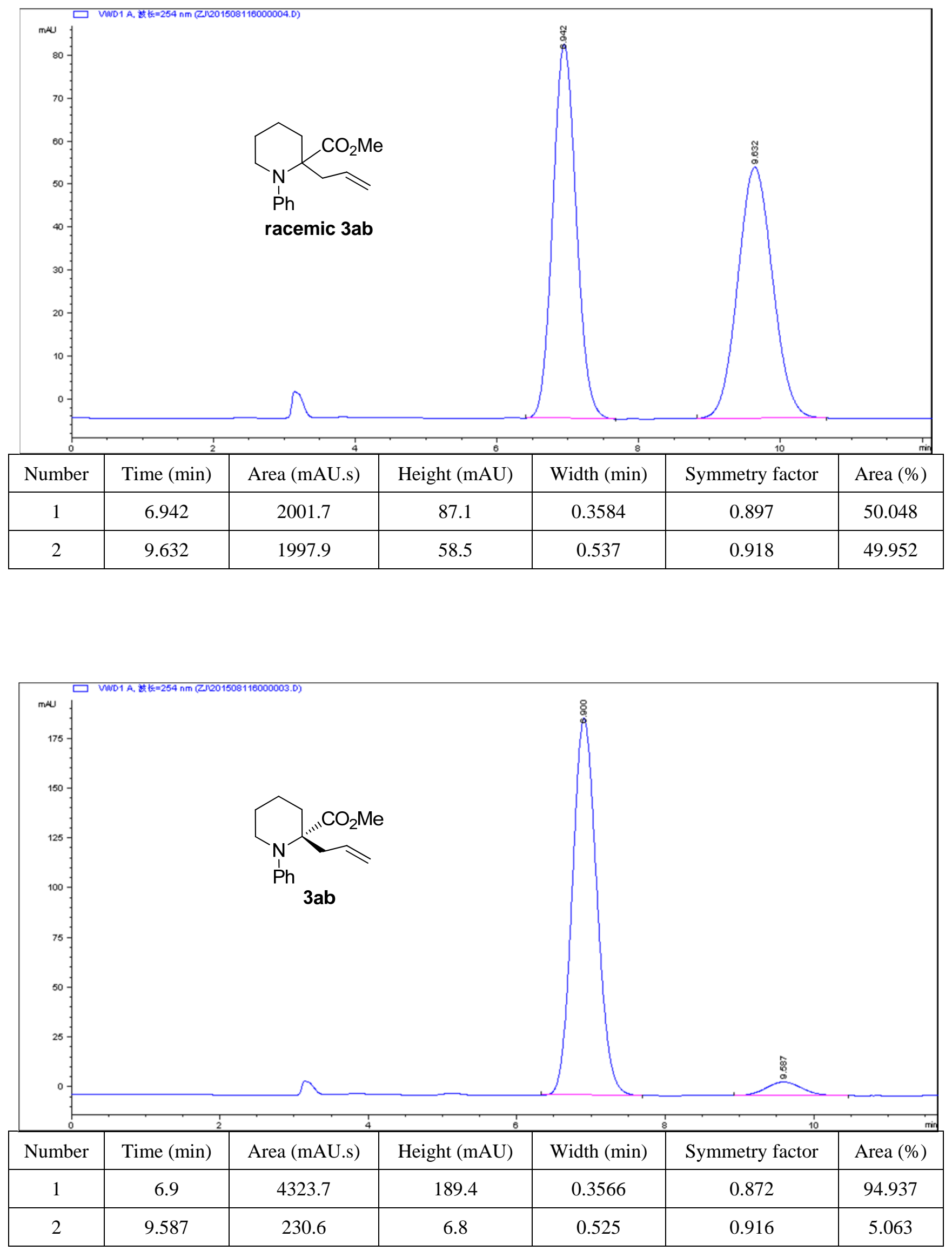


\section{Compound 6}

The crystal of $\mathbf{6}$ was obtained by leaving alone its solution in hexane and ether at room temperature in the open air for five days. The absolute configuration of compound $\mathbf{6}$ was assigned to be $S$ by single crystal X-ray analysis. The crystal data of compound $\mathbf{6}$ have been deposited in CCDC with number 1450238.<smiles>C=CC[C@]1(C(=O)NCCNS(=O)(=O)c2ccc(Br)cc2)CCCN1c1ccccc1</smiles>

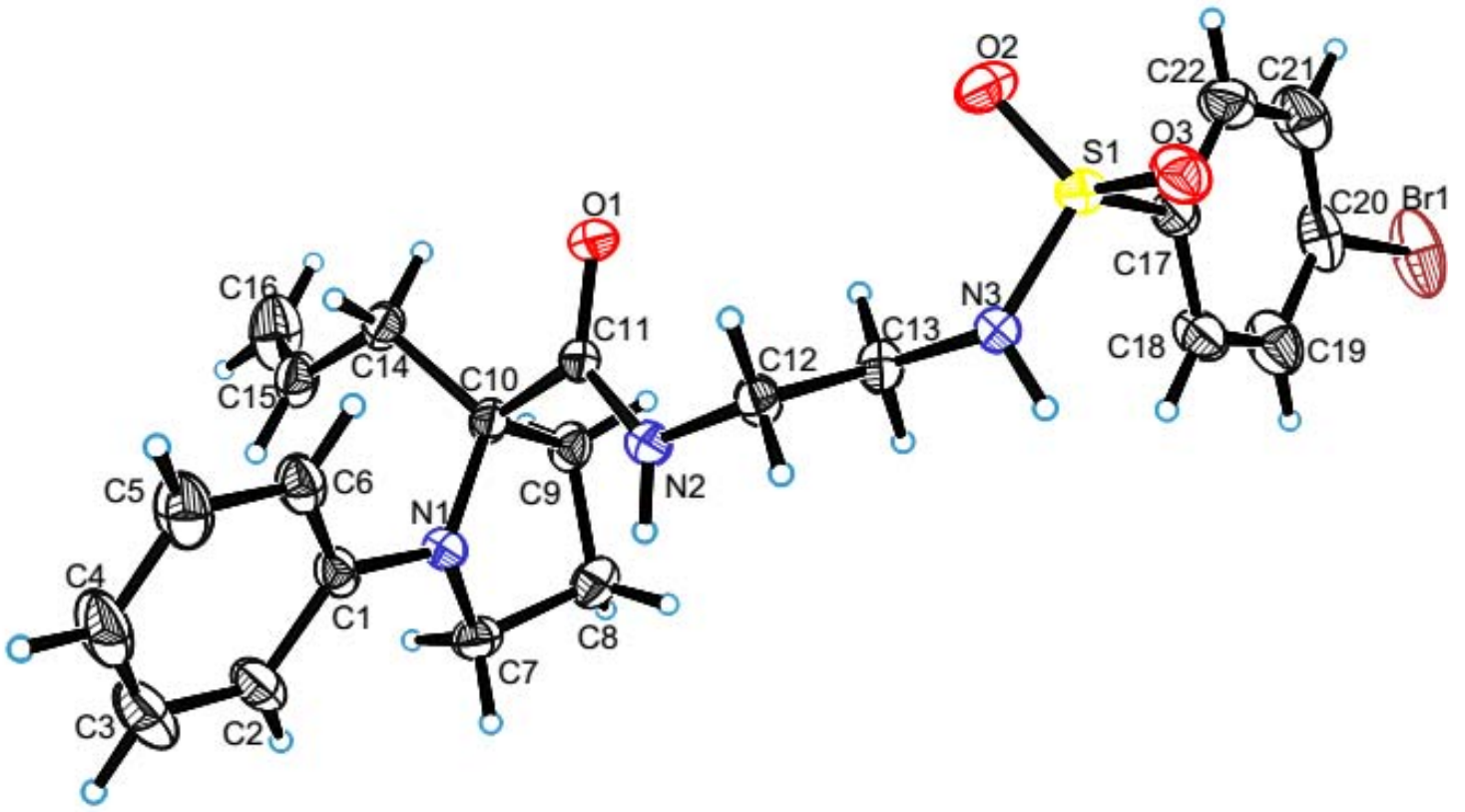

Table 1 Crystal data and structure refinement for ZJ-04-60.

Identification code

Empirical formula

Formula weight

Temperature/K

Crystal system

Space group
$\mathrm{a} / \AA ̊$
$\mathrm{b} / \AA$
$\mathrm{c} / \AA$
$\alpha /^{\circ}$

ZJ-04-60

$\mathrm{C}_{22} \mathrm{H}_{26} \mathrm{BrN}_{3} \mathrm{O}_{3} \mathrm{~S}$

492.43

289(2)

monoclinic

$\mathrm{P} 2{ }_{1}$

9.1897(2)

10.1219(3)

$12.6525(3)$

90 


$\begin{array}{ll}\beta /{ }^{\circ} & 95.598(2) \\ \gamma /{ }^{\circ} & 90 \\ \text { Volume } / \AA^{3} & 1171.29(5) \\ \mathrm{Z} & 2 \\ \rho_{\text {calc }} / \mathrm{cm}^{3} & 1.396 \\ \mu / \mathrm{mm}^{-1} & 3.448 \\ \mathrm{~F}(000) & 508.0 \\ \text { Crystal size } / \mathrm{mm}^{3} & 0.320 \times 0.280 \times 0.260 \\ \text { Radiation } & \mathrm{CuK} \alpha(\lambda=1.54184) \\ 2 \Theta \text { range for data collection/ } & 9.67 \text { to } 140.31 \\ \text { Index ranges } & -11 \leq \mathrm{h} \leq 11,-12 \leq \mathrm{k} \leq 8,-14 \leq 1 \leq 15 \\ \text { Reflections collected } & 4516 \\ \text { Independent reflections } & 2955\left[\mathrm{R}_{\text {int }}=0.0284, \mathrm{R}_{\text {sigma }}=0.0462\right] \\ \text { Data/restraints/parameters } & 2955 / 3 / 278 \\ \text { Goodness-of-fit on } \mathrm{F}^{2} & 1.048 \\ \text { Final R indexes }[\mathrm{I}>=2 \sigma(\mathrm{I})] & \mathrm{R}_{1}=0.0355, \mathrm{wR}_{2}=0.0934 \\ \text { Final R indexes [all data] } & \mathrm{R}_{1}=0.0377, \mathrm{wR}_{2}=0.0967 \\ \text { Largest diff. peak/hole / e } \AA^{-3} 0.24 /-0.23 \\ \text { Flack parameter } & -0.029(15)\end{array}$

Table 2 Fractional Atomic Coordinates $\left(\times 10^{4}\right)$ and Equivalent Isotropic Displacement Parameters $\left(\AA^{2} \times 10^{3}\right)$ for $\mathrm{ZJ-04-60} . \mathrm{U}_{\mathrm{eq}}$ is defined as $1 / 3$ of of the trace of the orthogonalised $U_{I J}$ tensor.

\begin{tabular}{lrrrr} 
Atom & \multicolumn{2}{c}{$\boldsymbol{x}$} & \multicolumn{1}{c}{$\boldsymbol{U}(\mathbf{e q})$} \\
Br1 & $4349.3(6)$ & $8012.5(16)$ & $-6326.6(4)$ & $126.5(4)$ \\
S1 & $2633.1(10)$ & $6650.7(9)$ & $-1675.4(7)$ & $53.3(2)$ \\
O1 & $6912(4)$ & $5361(3)$ & $949(3)$ & $70.1(9)$ \\
N3 & $3404(3)$ & $7656(3)$ & $-823(3)$ & $50.7(7)$ \\
O2 & $5358(3)$ & $-1404(3)$ & $80.6(10)$ \\
N1 & $3142(5)$ & $7760(3)$ & $2302(2)$ & $49.2(6)$ \\
O3 & $9329(3)$ & $6919(3)$ & $-1747(3)$ & $67.9(8)$ \\
N2 & $1102(3)$ & $7517(3)$ & $1224(3)$ & $52.2(7)$ \\
C10 & $6607(3)$ & $6626(4)$ & $1592(3)$ & $46.6(7)$ \\
C12 & $9063(4)$ & $7533(4)$ & $780(3)$ & $53.0(8)$ \\
C14 & $5089(4)$ & $5298(4)$ & $2025(3)$ & $55.7(9)$ \\
C11 & $6444(4)$ & $1245(3)$ & $49.1(8)$ \\
C2 & $9723(5)$ & $8492(5)$ & $4108(4)$ & $74.5(13)$ \\
C6 & $7414(4)$ & $6838(5)$ & $3790(3)$ & $64.5(11)$ \\
C1 & $10022(5)$ & $7686(4)$ & $3386(3)$ & $53.6(8)$ \\
C9 & $8180(5)$ & $7050(5)$ & $591(3)$ & $59.6(10)$ \\
C13 & $9180(4)$ & $7552(4)$ & $-412(3)$ & $55.1(8)$ \\
C4 & $9812(5)$ & $7601(9)$ & $5565(4)$ & $101(2)$
\end{tabular}




$\begin{array}{lrrrr}\text { C8 } & 10058(5) & 8513(5) & 700(4) & 67.9(11) \\ \text { C22 } & 2927(6) & 6104(6) & -3738(4) & 76.6(13) \\ \text { C15 } & 11292(5) & 5418(5) & 2427(4) & 69.6(11) \\ \text { C18 } & 3825(5) & 8233(6) & -3135(4) & 71.7(12) \\ \text { C7 } & 8687(4) & 1896(4) & 61.8(10) \\ \text { C17 } & 10355(4) & 7027(4) & -2937(3) & 54.0(9) \\ \text { C3 } & 3221(4) & 5444(9) & 5177(5) & 100(2) \\ \text { C19 } & 9867(7) & 8513(8) & -4146(4) & 91.5(18) \\ \text { C20 } & 4187(6) & 7592(8) & -4927(3) & 79.8(16) \\ \text { C21 } & 3914(5) & 6391(8) & -4749(5) & 92(2) \\ \text { C16 } & 3282(7) & 4715(9) & 2105(6) & 111(3) \\ \text { C5 } & 12342(7) & 6800(7) & 4874(4) & 87.1(17)\end{array}$

Table 3 Anisotropic Displacement Parameters $\left(\AA^{2} \times 10^{3}\right)$ for ZJ-04-60. The Anisotropic displacement factor exponent takes the form: $-2 \pi^{2}\left[h^{2} a *{ }^{2} U_{11}+2 h k a * b * U_{12}+\ldots\right]$.

\begin{tabular}{lrrrrrr} 
Atom & \multicolumn{1}{c}{$\mathbf{U}_{\mathbf{1 1}}$} & \multicolumn{1}{c}{$\mathbf{U}_{\mathbf{2 2}}$} & \multicolumn{1}{c}{$\mathbf{U}_{\mathbf{3 3}}$} & \multicolumn{1}{c}{$\mathbf{U}_{\mathbf{2 3}}$} & \multicolumn{1}{c}{$\mathbf{U}_{\mathbf{1 3}}$} & \multicolumn{1}{c}{$\mathbf{U}_{\mathbf{1 2}}$} \\
$\mathrm{Br} 1$ & $78.0(3)$ & $247.2(12)$ & $55.0(3)$ & $11.4(5)$ & $10.2(2)$ & $1.7(5)$ \\
$\mathrm{S} 1$ & $61.2(5)$ & $40.9(4)$ & $56.7(5)$ & $1.0(4)$ & $-0.3(3)$ & $-7.2(4)$ \\
O1 & $81.4(19)$ & $38.7(14)$ & $84(2)$ & $1.7(14)$ & $-22.2(16)$ & $-3.2(13)$ \\
$\mathrm{N} 3$ & $55.6(15)$ & $42.9(14)$ & $51.0(15)$ & $-0.8(14)$ & $-7.7(12)$ & $-1.0(13)$ \\
O2 & $109(3)$ & $45.4(16)$ & $85(2)$ & $5.6(16)$ & $1.4(19)$ & $-1.1(16)$ \\
$\mathrm{N} 1$ & $53.0(14)$ & $45.0(15)$ & $48.1(14)$ & $-4.6(13)$ & $-2.9(11)$ & $-0.2(13)$ \\
O3 & $56.2(15)$ & $75(2)$ & $71.6(18)$ & $-1.6(14)$ & $1.3(13)$ & $-15.2(13)$ \\
N2 & $55.1(16)$ & $44.2(16)$ & $54.7(17)$ & $-4.3(12)$ & $-7.9(12)$ & $1.0(13)$ \\
C10 & $56.4(17)$ & $44.3(16)$ & $38.6(15)$ & $2.3(15)$ & $1.6(12)$ & $7.1(16)$ \\
C12 & $50.0(17)$ & $57(2)$ & $50.4(19)$ & $0.7(16)$ & $-2.1(14)$ & $-0.7(16)$ \\
C14 & $70(2)$ & $50(2)$ & $47.1(19)$ & $4.4(16)$ & $4.5(16)$ & $12.7(17)$ \\
C11 & $65(2)$ & $40.8(18)$ & $39.9(16)$ & $1.8(13)$ & $-4.7(14)$ & $1.2(15)$ \\
C2 & $75(2)$ & $82(3)$ & $64(3)$ & $-27(2)$ & $-11(2)$ & $6(2)$ \\
C6 & $69(2)$ & $78(3)$ & $46(2)$ & $-6.2(19)$ & $2.7(17)$ & $11(2)$ \\
C1 & $50.4(16)$ & $59(2)$ & $49.6(18)$ & $-8.3(17)$ & $-4.9(13)$ & $13.3(16)$ \\
C9 & $74(2)$ & $64(2)$ & $42.4(18)$ & $6.8(16)$ & $10.1(17)$ & $4.6(18)$ \\
C13 & $50.5(17)$ & $65(2)$ & $49.1(18)$ & $6.7(17)$ & $0.3(13)$ & $1.1(16)$ \\
C4 & $123(5)$ & $137(6)$ & $43(2)$ & $-26(3)$ & $2(3)$ & $29(5)$ \\
C8 & $70(2)$ & $64(3)$ & $71(3)$ & $16(2)$ & $11.4(19)$ & $1(2)$ \\
C22 & $91(3)$ & $66(3)$ & $71(3)$ & $-14(2)$ & $3(2)$ & $-7(3)$ \\
C15 & $69(2)$ & $75(3)$ & $62(2)$ & $6(2)$ & $-4.2(19)$ & $21(2)$ \\
C18 & $81(3)$ & $78(3)$ & $55(2)$ & $-4(2)$ & $-0.4(18)$ & $-24(2)$ \\
C7 & $59(2)$ & $48(2)$ & $78(3)$ & $0.5(19)$ & $3.1(18)$ & $-4.2(16)$ \\
C17 & $51.2(18)$ & $55(2)$ & $54(2)$ & $-6.1(16)$ & $-0.3(15)$ & $1.2(15)$ \\
C3 & $102(4)$ & $130(6)$ & $65(3)$ & $-43(4)$ & $-7(3)$ & $8(4)$ \\
C19 & $85(3)$ & $125(5)$ & $64(3)$ & $12(3)$ & $3(2)$ & $-29(3)$
\end{tabular}




$\begin{array}{llllrrr}\mathrm{C} 20 & 55(2) & 135(5) & 50(2) & -1(3) & 2.5(16) & 10(3) \\ \mathrm{C} 21 & 89(3) & 119(6) & 68(3) & -32(3) & 1(3) & 9(3) \\ \mathrm{C} 16 & 82(3) & 157(7) & 93(4) & 5(4) & 5(3) & 53(4) \\ \mathrm{C} 5 & 94(3) & 117(5) & 53(2) & -3(3) & 17(2) & 17(3)\end{array}$

Table 4 Bond Lengths for ZJ-04-60.

\begin{tabular}{|c|c|c|c|c|c|}
\hline \multicolumn{2}{|c|}{ Atom Atom } & \multirow{2}{*}{$\begin{array}{r}\text { Length/Å } \\
1.901(5)\end{array}$} & \multicolumn{2}{|c|}{ Atom Atom } & \multirow{2}{*}{$\begin{array}{c}\text { Length/Å } \\
1.487(6)\end{array}$} \\
\hline $\mathrm{Br} 1$ & $\mathrm{C} 20$ & & $\mathrm{C} 14$ & $\mathrm{C} 15$ & \\
\hline S1 & $\mathrm{O} 2$ & $1.420(4)$ & $\mathrm{C} 2$ & $\mathrm{C} 3$ & $1.374(8)$ \\
\hline S1 & $\mathrm{O} 3$ & $1.428(3)$ & $\mathrm{C} 2$ & $\mathrm{C} 1$ & $1.400(6)$ \\
\hline S1 & N3 & $1.597(3)$ & C6 & $\mathrm{C} 1$ & $1.392(6)$ \\
\hline S1 & $\mathrm{C} 17$ & $1.776(4)$ & $\mathrm{C} 6$ & $\mathrm{C} 5$ & $1.392(7)$ \\
\hline $\mathrm{O} 1$ & C11 & $1.234(5)$ & C9 & $\mathrm{C} 8$ & $1.502(6)$ \\
\hline N3 & $\mathrm{C} 13$ & $1.464(4)$ & $\mathrm{C} 4$ & $\mathrm{C} 3$ & $1.370(11)$ \\
\hline N1 & $\mathrm{C} 1$ & $1.393(5)$ & $\mathrm{C} 4$ & $\mathrm{C} 5$ & $1.377(9)$ \\
\hline N1 & $\mathrm{C} 7$ & $1.458(5)$ & $\mathrm{C} 8$ & $\mathrm{C} 7$ & $1.522(7)$ \\
\hline N1 & $\mathrm{C} 10$ & $1.464(5)$ & $\mathrm{C} 22$ & $\mathrm{C} 21$ & $1.382(9)$ \\
\hline $\mathrm{N} 2$ & $\mathrm{C} 11$ & $1.314(5)$ & $\mathrm{C} 22$ & $\mathrm{C} 17$ & $1.385(6)$ \\
\hline $\mathrm{N} 2$ & $\mathrm{C} 12$ & $1.453(4)$ & $\mathrm{C} 15$ & $\mathrm{C} 16$ & $1.296(8)$ \\
\hline $\mathrm{C} 10$ & C11 & $1.547(5)$ & C18 & $\mathrm{C} 17$ & $1.373(7)$ \\
\hline $\mathrm{C} 10$ & $\mathrm{C} 14$ & $1.552(5)$ & $\mathrm{C} 18$ & C19 & $1.382(7)$ \\
\hline $\mathrm{C} 10$ & C9 & $1.559(5)$ & C19 & $\mathrm{C} 20$ & $1.365(9)$ \\
\hline $\mathrm{C} 12$ & $\mathrm{C} 13$ & $1.500(5)$ & $\mathrm{C} 20$ & $\mathrm{C} 21$ & $1.375(10)$ \\
\hline
\end{tabular}

Table 5 Bond Angles for ZJ-04-60.

\begin{tabular}{|c|c|c|c|c|c|c|c|}
\hline \multicolumn{3}{|c|}{ Atom Atom Atom } & \multirow{2}{*}{$\begin{array}{l}\text { Angle }^{\circ} \\
\qquad 119.4(2)\end{array}$} & \multicolumn{3}{|c|}{ Atom Atom Atom } & \multirow{2}{*}{$\begin{array}{l}\text { Angle }^{\circ} \\
\qquad 120.7(5)\end{array}$} \\
\hline $\mathrm{O} 2$ & $\mathrm{~S} 1$ & $\mathrm{O} 3$ & & $\mathrm{C} 1$ & C6 & $\mathrm{C} 5$ & \\
\hline $\mathrm{O} 2$ & $\mathrm{~S} 1$ & N3 & $108.0(2)$ & $\mathrm{C} 6$ & $\mathrm{C} 1$ & N1 & $121.8(3)$ \\
\hline $\mathrm{O} 3$ & S1 & $\mathrm{N} 3$ & $106.92(19)$ & C6 & $\mathrm{C} 1$ & $\mathrm{C} 2$ & $117.5(4)$ \\
\hline $\mathrm{O} 2$ & S1 & $\mathrm{C} 17$ & $107.0(2)$ & $\mathrm{N} 1$ & $\mathrm{C} 1$ & $\mathrm{C} 2$ & $120.7(4)$ \\
\hline $\mathrm{O} 3$ & S1 & $\mathrm{C} 17$ & $106.66(19)$ & $\mathrm{C} 8$ & C9 & $\mathrm{C} 10$ & $105.7(4)$ \\
\hline N3 & $\mathrm{S} 1$ & $\mathrm{C} 17$ & $108.46(18)$ & N3 & $\mathrm{C} 13$ & $\mathrm{C} 12$ & $110.2(3)$ \\
\hline $\mathrm{C} 13$ & N3 & $\mathrm{S} 1$ & $123.0(3)$ & $\mathrm{C} 3$ & $\mathrm{C} 4$ & $\mathrm{C} 5$ & $119.5(5)$ \\
\hline $\mathrm{C} 1$ & N1 & $\mathrm{C} 7$ & $120.7(3)$ & $\mathrm{C} 9$ & $\mathrm{C} 8$ & $\mathrm{C} 7$ & $102.5(4)$ \\
\hline $\mathrm{C} 1$ & N1 & $\mathrm{C} 10$ & $122.4(3)$ & $\mathrm{C} 21$ & $\mathrm{C} 22$ & $\mathrm{C} 17$ & $119.2(6)$ \\
\hline $\mathrm{C} 7$ & N1 & $\mathrm{C} 10$ & $111.3(3)$ & $\mathrm{C} 16$ & $\mathrm{C} 15$ & $\mathrm{C} 14$ & $125.1(6)$ \\
\hline $\mathrm{C} 11$ & $\mathrm{~N} 2$ & $\mathrm{C} 12$ & $122.4(3)$ & $\mathrm{C} 17$ & $\mathrm{C} 18$ & $\mathrm{C} 19$ & $119.3(5)$ \\
\hline N1 & $\mathrm{C} 10$ & $\mathrm{C} 11$ & $111.6(3)$ & $\mathrm{N} 1$ & $\mathrm{C} 7$ & $\mathrm{C} 8$ & $102.5(3)$ \\
\hline N1 & $\mathrm{C} 10$ & $\mathrm{C} 14$ & $115.2(3)$ & C18 & C17 & $\mathrm{C} 22$ & $121.3(4)$ \\
\hline $\mathrm{C} 11$ & $\mathrm{C} 10$ & $\mathrm{C} 14$ & $109.6(3)$ & C18 & C17 & $\mathrm{S} 1$ & $121.2(3)$ \\
\hline N1 & $\mathrm{C} 10$ & C9 & $102.9(3)$ & $\mathrm{C} 22$ & $\mathrm{C} 17$ & $\mathrm{~S} 1$ & $117.2(4)$ \\
\hline C11 & $\mathrm{C} 10$ & C9 & $107.5(3)$ & $\mathrm{C} 4$ & C3 & $\mathrm{C} 2$ & $120.7(5)$ \\
\hline
\end{tabular}




$\begin{array}{llllllll}\mathrm{C} 14 & \mathrm{C} 10 & \mathrm{C} 9 & 109.7(3) & \mathrm{C} 20 & \mathrm{C} 19 & \mathrm{C} 18 & 119.1(6) \\ \mathrm{N} 2 & \mathrm{C} 12 & \mathrm{C} 13 & 112.1(3) & \mathrm{C} 19 & \mathrm{C} 20 & \mathrm{C} 21 & 122.4(5) \\ \mathrm{C} 15 & \mathrm{C} 14 & \mathrm{C} 10 & 112.5(4) & \mathrm{C} 19 & \mathrm{C} 20 & \mathrm{Br} 1 & 118.8(5) \\ \mathrm{O} 1 & \mathrm{C} 11 & \mathrm{~N} 2 & 122.3(4) & \mathrm{C} 21 & \mathrm{C} 20 & \mathrm{Br} 1 & 118.8(4) \\ \mathrm{O} 1 & \mathrm{C} 11 & \mathrm{C} 10 & 121.3(4) & \mathrm{C} 20 & \mathrm{C} 21 & \mathrm{C} 22 & 118.7(5) \\ \mathrm{N} 2 & \mathrm{C} 11 & \mathrm{C} 10 & 116.3(3) & \mathrm{C} 4 & \mathrm{C} 5 & \mathrm{C} 6 & 120.4(6) \\ \mathrm{C} 3 & \mathrm{C} 2 & \mathrm{C} 1 & 121.2(6) & & & & \end{array}$

Table 6 Hydrogen Atom Coordinates $\left(\AA \times 10^{4}\right)$ and Isotropic Displacement Parameters $\left(\AA^{2} \times 10^{3}\right)$ for $\mathrm{ZJ}-04-60$.

\begin{tabular}{|c|c|c|c|c|}
\hline Atom & $x$ & & $\mathbf{z}$ & $\mathbf{U}(\mathbf{e q})$ \\
\hline $\mathrm{H} 12 \mathrm{~A}$ & 4611 & 8307 & 1037 & 64 \\
\hline H12B & 4596 & 6758 & 1021 & 64 \\
\hline H14A & 9176 & 4992 & 2596 & 67 \\
\hline H14B & 9621 & 4642 & 1464 & 67 \\
\hline $\mathrm{H} 2$ & 10697 & 9071 & 3860 & 89 \\
\hline H6 & 7604 & 6290 & 3331 & 77 \\
\hline H9A & 10733 & 6590 & 564 & 72 \\
\hline H9B & 9186 & 6853 & -52 & 72 \\
\hline H13A & 5484 & 8297 & -658 & 66 \\
\hline H13B & 5359 & 6749 & -675 & 66 \\
\hline $\mathrm{H} 4$ & 8788 & 7569 & 6289 & 122 \\
\hline H8A & 9200 & 9005 & 421 & 82 \\
\hline H8B & 10889 & 8792 & 338 & 82 \\
\hline $\mathrm{H} 22$ & 2496 & 5300 & -3596 & 92 \\
\hline H15 & 11540 & 6047 & 2950 & 84 \\
\hline H18 & 3989 & 8853 & -2594 & 86 \\
\hline H7A & 11357 & 8462 & 2141 & 74 \\
\hline H7B & 10159 & 9586 & 2107 & 74 \\
\hline H3 & 10436 & 8990 & 5641 & 120 \\
\hline H19 & 4610 & 9319 & -4291 & 110 \\
\hline $\mathrm{H} 21$ & 3097 & 5785 & -5298 & 111 \\
\hline H16A & 12138 & 4076 & 1584 & 134 \\
\hline H16B & 13299 & 4849 & 2396 & 134 \\
\hline H5 & 7356 & 6232 & 5134 & 105 \\
\hline $\mathrm{H} 2 \mathrm{~A}$ & $6940(70)$ & $8320(30)$ & $1390(50)$ & 105 \\
\hline $\mathrm{H} 3 \mathrm{~A}$ & $3200(70)$ & $8520(30)$ & $-900(60)$ & 105 \\
\hline
\end{tabular}




\section{Compound 7}

The crystal of 7 was obtained by leaving alone its solution in hexane and chloroform at room temperature in the open air for two days. The absolute configuration of compound 7 was assigned to be $S$ by single crystal X-ray analysis. The crystal data of compound 7 have been deposited in CCDC with number 1450237.

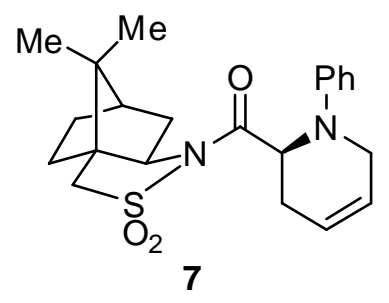

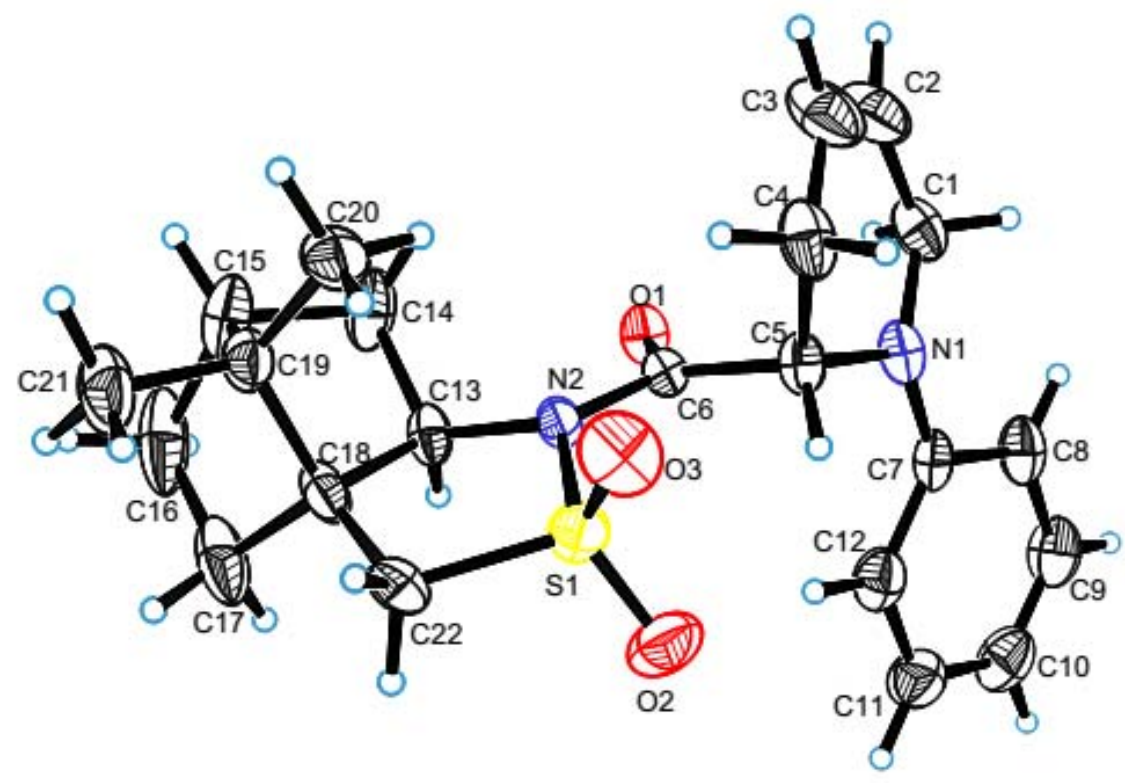

Table 1 Crystal data and structure refinement for ZJ-04-57.

Identification code

Empirical formula

Formula weight

Temperature/K

Crystal system

Space group

$\mathrm{a} / \AA$
$\mathrm{b} / \AA$
$\mathrm{c} / \AA$
$\alpha{ }^{\circ}$
$\beta /{ }^{\circ}$
ZJ-04-57

$\mathrm{C}_{23} \mathrm{H}_{29} \mathrm{Cl}_{3} \mathrm{~N}_{2} \mathrm{O}_{3} \mathrm{~S}$

519.89

288(2)

orthorhombic

$\mathrm{P} 2{ }_{1} 2_{1} 2_{1}$

10.8104(3)

$11.1726(3)$

21.1349(5)

90

90 


$\begin{array}{ll}\gamma^{\circ} & 90 \\ \text { Volume } / \AA^{3} & 2552.68(12) \\ \mathrm{Z} & 4 \\ \rho_{\text {calc }} / \mathrm{cm}^{3} & 1.353 \\ \mu / \mathrm{mm}^{-1} & 4.236 \\ \mathrm{~F}(000) & 1088.0 \\ \text { Crystal size } / \mathrm{mm}^{3} & 0.310 \times 0.300 \times 0.240 \\ \text { Radiation } & \mathrm{CuK} \alpha(\lambda=1.54184) \\ 2 \Theta \text { range for data collection } /{ }^{\circ} 8.952 \text { to } 139.802 \\ \text { Index ranges } & -12 \leq \mathrm{h} \leq 13,-11 \leq \mathrm{k} \leq 13,-15 \leq 1 \leq 25 \\ \text { Reflections collected } & 9540 \\ \text { Independent reflections } & 4721\left[\mathrm{R}_{\text {int }}=0.0274, \mathrm{R}_{\text {sigma }}=0.0343\right] \\ \text { Data/restraints/parameters } & 4721 / 0 / 292 \\ \text { Goodness-of-fit on } \mathrm{F}^{2} & 1.060 \\ \text { Final R indexes [I }>=2 \sigma(\mathrm{I})] & \mathrm{R}_{1}=0.0771, \mathrm{wR}_{2}=0.1938 \\ \text { Final R indexes [all data] } & \mathrm{R}_{1}=0.0815, \mathrm{wR}_{2}=0.1985 \\ \text { Largest diff. peak/hole / e } \AA^{-3} 0.78 /-0.52 \\ \text { Flack parameter } & 0.007(8)\end{array}$

Table 2 Fractional Atomic Coordinates $\left(\times 10^{4}\right)$ and Equivalent Isotropic Displacement Parameters $\left(\AA^{2} \times 10^{3}\right)$ for $\mathrm{ZJ-04-57} . \mathrm{U}_{\text {eq }}$ is defined as $1 / 3$ of of the trace of the orthogonalised $U_{I J}$ tensor.

\begin{tabular}{lrrrr} 
Atom & \multicolumn{1}{r}{$\boldsymbol{x}$} & \multicolumn{1}{c}{$\boldsymbol{U}(\mathbf{e q})$} \\
$\mathrm{S} 1$ & $11219.2(15)$ & $7223.7(12)$ & $3314.2(9)$ & $67.5(5)$ \\
O1 & $11542(3)$ & $10580(3)$ & $3055.9(19)$ & $55.5(9)$ \\
$\mathrm{Cl3}$ & $12015(5)$ & $14008(3)$ & $3781(3)$ & $186.0(19)$ \\
C12 & $9527(4)$ & $13424(4)$ & $3685(3)$ & $189(2)$ \\
C11 & $12487(5)$ & $4728.1(16)$ & $229(3)$ \\
N2 & $8729(3)$ & $3327(2)$ & $45.5(9)$ \\
O2 & $10938(4)$ & $6912(5)$ & $2734(3)$ & $100.4(19)$ \\
O3 & $11810(6)$ & $6886(4)$ & $3888(3)$ & $93.4(17)$ \\
N1 & $11821(6)$ & $10016(5)$ & $3001(3)$ & $59.9(12)$ \\
C13 & $14050(4)$ & $9025(6)$ & $3213(3)$ & $54.8(13)$ \\
C7 & $9627(5)$ & $2348(3)$ & $57.3(14)$ \\
C11 & $9910(5)$ & $1353(4)$ & $84(2)$ \\
C6 & $8945(8)$ & $3210(2)$ & $44.0(11)$ \\
C5 & $14194(5)$ & $9586(4)$ & $3320(3)$ & $54.5(13)$ \\
C8 & $13782(8)$ & $9222(5)$ & $2026(4)$ & $72.2(18)$ \\
C18 & $11844(4)$ & $10650(7)$ & $3409(3)$ & $62.7(15)$ \\
C19 & $13188(5)$ & $7905(6)$ & $4093(3)$ & $62.3(15)$ \\
C1 & $15035(6)$ & $8209(6)$ & $3282(4)$ & $73.2(17)$ \\
C14 & $8861(5)$ & $11192(6)$ & $3606(4)$ & $83(2)$
\end{tabular}




$\begin{array}{lrrrr}\text { C10 } & 14624(8) & 9692(8) & 1047(4) & 92(2) \\ \text { C17 } & 7685(7) & 7962(11) & 3034(4) & 110(4) \\ \text { C9 } & 15230(7) & 10516(8) & 1383(4) & 87(2) \\ \text { C4 } & 9180(8) & 4039(3) & 78(2) \\ \text { C15 } & 73447(6) & 9432(9) & 3888(5) & 94(3) \\ \text { C20 } & 8227(8) & 4596(3) & 78(2) \\ \text { C21 } & 9467(7) & 7365(10) & 4345(4) & 98(3) \\ \text { C16 } & 7431(7) & 9074(12) & 3323(5) & 129(5) \\ \text { C12 } & 7074(7) & 9064(7) & 1990(4) & 72.7(17) \\ \text { C22 } & 13575(6) & 6786(6) & 3303(4) & 82(2) \\ \text { C23 } & 9633(7) & 12925(7) & 3978(5) & 105(3) \\ \text { C2 } & 10947(10) & 11243(9) & 3970(4) & 98(3) \\ \text { C3 } & 14031(9) & 10371(9) & 4314(4) & 102(3)\end{array}$

Table 3 Anisotropic Displacement Parameters $\left(\AA^{2} \times 10^{3}\right)$ for ZJ-04-57. The Anisotropic displacement factor exponent takes the form: $-2 \pi^{2}\left[h^{2} a *{ }^{2} U_{11}+2 h k a * b * U_{12}+\ldots\right]$.

\begin{tabular}{|c|c|c|c|c|c|c|}
\hline Atom & $\mathbf{U}_{11}$ & $\mathbf{U}_{22}$ & $\mathbf{U}_{33}$ & $\mathbf{U}_{23}$ & $\mathbf{U}_{13}$ & $\mathbf{U}_{12}$ \\
\hline S1 & $68.6(9)$ & $41.4(6)$ & $92.5(11)$ & $-7.5(7)$ & $14.2(8)$ & $-4.2(7)$ \\
\hline $\mathrm{O} 1$ & 47.9(19) & $44.6(19)$ & $74(2)$ & $17.1(17)$ & $-2.2(17)$ & $3.1(16)$ \\
\hline $\mathrm{Cl} 3$ & $196(4)$ & 88.1(18) & $274(5)$ & $23(3)$ & $-1(4)$ & $-37(2)$ \\
\hline $\mathrm{Cl} 2$ & $155(3)$ & $127(3)$ & $286(6)$ & $39(3)$ & $-25(3)$ & $13(2)$ \\
\hline $\mathrm{Cl1}$ & $328(7)$ & $258(5)$ & $102(2)$ & $37(3)$ & $69(3)$ & $86(5)$ \\
\hline N2 & $39(2)$ & $42(2)$ & $55(2)$ & $-0.8(18)$ & $-1.5(18)$ & $-2.0(16)$ \\
\hline $\mathrm{O} 2$ & $115(5)$ & $68(3)$ & $118(4)$ & $-27(3)$ & $45(4)$ & $-10(3)$ \\
\hline $\mathrm{O} 3$ & $105(4)$ & $60(3)$ & $115(4)$ & $25(3)$ & $-23(3)$ & $16(3)$ \\
\hline N1 & $40(2)$ & $63(3)$ & $76(3)$ & $15(2)$ & $-1(2)$ & $-6(2)$ \\
\hline C13 & $37(2)$ & $71(4)$ & $56(3)$ & $19(3)$ & $-5(2)$ & $-4(2)$ \\
\hline $\mathrm{C} 7$ & $35(2)$ & $59(3)$ & $77(4)$ & $14(3)$ & $8(2)$ & $5(2)$ \\
\hline $\mathrm{C} 11$ & $84(5)$ & $86(5)$ & $81(5)$ & $-10(4)$ & $17(4)$ & $-7(4)$ \\
\hline C6 & $40(2)$ & $45(3)$ & $47(3)$ & $1(2)$ & $-2(2)$ & $-4.3(19)$ \\
\hline $\mathrm{C} 5$ & $41(2)$ & $56(3)$ & $66(3)$ & $13(3)$ & $-1(2)$ & $2(2)$ \\
\hline $\mathrm{C} 8$ & $49(3)$ & $72(4)$ & $95(5)$ & $22(4)$ & $7(3)$ & $-6(3)$ \\
\hline $\mathrm{C} 18$ & $46(3)$ & $88(4)$ & $54(3)$ & $9(3)$ & $-11(2)$ & $-25(3)$ \\
\hline C19 & $50(3)$ & $78(4)$ & $59(3)$ & $9(3)$ & $5(3)$ & $-12(3)$ \\
\hline $\mathrm{C} 1$ & $61(4)$ & $73(4)$ & $85(4)$ & $12(4)$ & $-9(3)$ & $-17(3)$ \\
\hline $\mathrm{C} 14$ & $53(3)$ & $68(4)$ & $128(6)$ & $30(4)$ & $21(4)$ & $16(3)$ \\
\hline $\mathrm{C} 10$ & $94(6)$ & $95(6)$ & $86(5)$ & $6(4)$ & $34(5)$ & $4(5)$ \\
\hline $\mathrm{C} 17$ & $55(4)$ & $192(11)$ & $82(5)$ & $43(6)$ & $-24(4)$ & $-37(6)$ \\
\hline C9 & $72(5)$ & $94(6)$ & $95(5)$ & $28(5)$ & $27(4)$ & $-6(4)$ \\
\hline $\mathrm{C} 4$ & $54(3)$ & $103(6)$ & $77(4)$ & $39(4)$ & $-13(3)$ & $-2(4)$ \\
\hline C15 & $55(4)$ & $107(6)$ & $120(6)$ & $28(5)$ & $30(4)$ & $15(4)$ \\
\hline $\mathrm{C} 20$ & $76(5)$ & $111(6)$ & $48(3)$ & $5(3)$ & $-7(3)$ & $-17(4)$ \\
\hline
\end{tabular}




$\begin{array}{lrrrrrr}\mathrm{C} 21 & 70(4) & 142(8) & 82(5) & 28(5) & 13(4) & -36(5) \\ \mathrm{C} 16 & 40(3) & 206(12) & 141(8) & 83(9) & -5(5) & 4(5) \\ \mathrm{C} 12 & 63(4) & 72(4) & 84(4) & 7(3) & 14(3) & -1(3) \\ \mathrm{C} 22 & 79(4) & 69(4) & 97(5) & -27(4) & 16(4) & -36(4) \\ \mathrm{C} 23 & 127(7) & 60(4) & 127(7) & -11(4) & 19(6) & 5(5) \\ \mathrm{C} 2 & 105(6) & 113(7) & 75(5) & 0(5) & -25(4) & -40(6) \\ \mathrm{C} 3 & 112(7) & 126(7) & 67(4) & 11(5) & -33(4) & -38(6)\end{array}$

Table 4 Bond Lengths for ZJ-04-57.

\begin{tabular}{|c|c|c|c|c|c|}
\hline \multicolumn{2}{|c|}{ Atom Atom } & \multirow{2}{*}{$\begin{array}{r}\text { Length/ } \AA \AA \\
1.426(5)\end{array}$} & \multicolumn{2}{|c|}{ Atom Atom } & \multirow{2}{*}{ 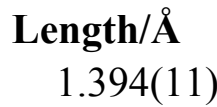 } \\
\hline S1 & $\mathrm{O} 2$ & & $\mathrm{C} 11$ & $\mathrm{C} 10$ & \\
\hline S1 & $\mathrm{O} 3$ & $1.427(6)$ & $\mathrm{C} 6$ & $\mathrm{C} 5$ & $1.527(7)$ \\
\hline S1 & N2 & $1.709(4)$ & $\mathrm{C} 5$ & $\mathrm{C} 4$ & $1.547(9)$ \\
\hline S1 & $\mathrm{C} 22$ & $1.783(7)$ & $\mathrm{C} 8$ & C9 & $1.384(11)$ \\
\hline O1 & C6 & $1.202(6)$ & C18 & $\mathrm{C} 17$ & $1.499(8)$ \\
\hline $\mathrm{Cl3}$ & $\mathrm{C} 23$ & $1.724(10)$ & $\mathrm{C} 18$ & $\mathrm{C} 22$ & $1.520(10)$ \\
\hline $\mathrm{Cl} 2$ & $\mathrm{C} 23$ & $1.747(11)$ & $\mathrm{C} 18$ & C19 & $1.555(9)$ \\
\hline $\mathrm{Cl1}$ & $\mathrm{C} 23$ & $1.660(11)$ & C19 & $\mathrm{C} 21$ & $1.533(9)$ \\
\hline N2 & C6 & $1.393(6)$ & C19 & C15 & $1.534(11)$ \\
\hline N2 & $\mathrm{C} 13$ & $1.474(6)$ & C19 & $\mathrm{C} 20$ & $1.541(8)$ \\
\hline N1 & $\mathrm{C} 7$ & $1.395(8)$ & $\mathrm{C} 1$ & $\mathrm{C} 2$ & $1.484(11)$ \\
\hline N1 & $\mathrm{C} 5$ & $1.452(7)$ & $\mathrm{C} 14$ & $\mathrm{C} 15$ & $1.555(10)$ \\
\hline N1 & $\mathrm{C} 1$ & $1.466(8)$ & $\mathrm{C} 10$ & C9 & $1.334(12)$ \\
\hline $\mathrm{C} 13$ & $\mathrm{C} 14$ & $1.502(10)$ & $\mathrm{C} 17$ & $\mathrm{C} 16$ & $1.533(17)$ \\
\hline $\mathrm{C} 13$ & $\mathrm{C} 18$ & $1.557(8)$ & $\mathrm{C} 4$ & $\mathrm{C} 3$ & $1.471(12)$ \\
\hline $\mathrm{C} 7$ & $\mathrm{C} 12$ & $1.383(10)$ & $\mathrm{C} 15$ & $\mathrm{C} 16$ & $1.562(15)$ \\
\hline $\mathrm{C} 7$ & $\mathrm{C} 8$ & $1.404(8)$ & $\mathrm{C} 2$ & C3 & $1.277(12)$ \\
\hline $\mathrm{C} 11$ & $\mathrm{C} 12$ & $1.371(10)$ & & & \\
\hline
\end{tabular}

Table 5 Bond Angles for ZJ-04-57.

\begin{tabular}{|c|c|c|c|c|c|c|c|}
\hline \multicolumn{3}{|c|}{ Atom Atom Atom } & \multirow{2}{*}{$\begin{array}{l}\text { Angle }^{\circ}{ }^{\circ} \\
\qquad 117.5(4)\end{array}$} & \multicolumn{3}{|c|}{ Atom Atom Atom } & \multirow{2}{*}{$\begin{array}{l}\text { Angle }^{\circ} \\
\qquad 118.6(6)\end{array}$} \\
\hline $\mathrm{O} 2$ & $\mathrm{~S} 1$ & $\mathrm{O} 3$ & & $\mathrm{C} 22$ & $\mathrm{C} 18$ & C19 & \\
\hline $\mathrm{O} 2$ & $\mathrm{~S} 1$ & $\mathrm{~N} 2$ & $109.5(3)$ & $\mathrm{C} 17$ & $\mathrm{C} 18$ & $\mathrm{C} 13$ & $106.1(6)$ \\
\hline $\mathrm{O} 3$ & $\mathrm{~S} 1$ & $\mathrm{~N} 2$ & $109.2(3)$ & $\mathrm{C} 22$ & $\mathrm{C} 18$ & $\mathrm{C} 13$ & $109.2(5)$ \\
\hline $\mathrm{O} 2$ & $\mathrm{~S} 1$ & $\mathrm{C} 22$ & $110.7(4)$ & C19 & $\mathrm{C} 18$ & $\mathrm{C} 13$ & $103.3(5)$ \\
\hline $\mathrm{O} 3$ & $\mathrm{~S} 1$ & $\mathrm{C} 22$ & $112.1(4)$ & $\mathrm{C} 21$ & C19 & $\mathrm{C} 15$ & $113.2(6)$ \\
\hline N2 & $\mathrm{S} 1$ & $\mathrm{C} 22$ & $95.7(3)$ & $\mathrm{C} 21$ & C19 & $\mathrm{C} 20$ & $106.3(5)$ \\
\hline C6 & $\mathrm{N} 2$ & $\mathrm{C} 13$ & $119.5(4)$ & $\mathrm{C} 15$ & C19 & $\mathrm{C} 20$ & $116.2(7)$ \\
\hline C6 & N2 & $\mathrm{S} 1$ & $123.3(3)$ & $\mathrm{C} 21$ & C19 & $\mathrm{C} 18$ & $113.5(6)$ \\
\hline $\mathrm{C} 13$ & $\mathrm{~N} 2$ & $\mathrm{~S} 1$ & $112.9(3)$ & $\mathrm{C} 15$ & C19 & $\mathrm{C} 18$ & $92.1(5)$ \\
\hline $\mathrm{C} 7$ & N1 & $\mathrm{C} 5$ & $118.6(5)$ & $\mathrm{C} 20$ & C19 & $\mathrm{C} 18$ & $115.4(5)$ \\
\hline $\mathrm{C} 7$ & N1 & $\mathrm{C} 1$ & $117.2(5)$ & N1 & $\mathrm{C} 1$ & $\mathrm{C} 2$ & $113.3(6)$ \\
\hline
\end{tabular}




\begin{tabular}{|c|c|c|c|c|c|c|c|}
\hline $\mathrm{C} 5$ & N1 & $\mathrm{C} 1$ & $118.4(5)$ & C13 & C14 & $\mathrm{C} 15$ & $101.6(6)$ \\
\hline N2 & $\mathrm{C} 13$ & $\mathrm{C} 14$ & $115.8(5)$ & C9 & $\mathrm{C} 10$ & $\mathrm{C} 11$ & $119.2(8)$ \\
\hline N2 & $\mathrm{C} 13$ & $\mathrm{C} 18$ & $106.7(4)$ & $\mathrm{C} 18$ & $\mathrm{C} 17$ & $\mathrm{C} 16$ & $100.9(8)$ \\
\hline $\mathrm{C} 14$ & $\mathrm{C} 13$ & C18 & $104.6(5)$ & $\mathrm{C} 10$ & C9 & $\mathrm{C} 8$ & $121.5(7)$ \\
\hline C12 & $\mathrm{C} 7$ & N1 & $123.0(6)$ & C3 & $\mathrm{C} 4$ & C5 & $113.0(6)$ \\
\hline $\mathrm{C} 12$ & $\mathrm{C} 7$ & $\mathrm{C} 8$ & $116.8(7)$ & C19 & $\mathrm{C} 15$ & $\mathrm{C} 14$ & $101.3(5)$ \\
\hline N1 & $\mathrm{C} 7$ & $\mathrm{C} 8$ & $120.1(6)$ & C19 & $\mathrm{C} 15$ & $\mathrm{C} 16$ & $101.4(8)$ \\
\hline $\mathrm{C} 12$ & $\mathrm{C} 11$ & $\mathrm{C} 10$ & $120.3(8)$ & $\mathrm{C} 14$ & $\mathrm{C} 15$ & $\mathrm{C} 16$ & $107.6(7)$ \\
\hline $\mathrm{O} 1$ & C6 & N2 & $119.5(4)$ & C17 & C16 & $\mathrm{C} 15$ & $104.9(7)$ \\
\hline $\mathrm{O} 1$ & C6 & $\mathrm{C} 5$ & $123.1(4)$ & $\mathrm{C} 11$ & $\mathrm{C} 12$ & $\mathrm{C} 7$ & $121.6(7)$ \\
\hline N2 & C6 & $\mathrm{C} 5$ & $117.3(4)$ & $\mathrm{C} 18$ & $\mathrm{C} 22$ & $\mathrm{~S} 1$ & $107.5(4)$ \\
\hline N1 & C5 & C6 & $112.2(4)$ & $\mathrm{Cl1}$ & $\mathrm{C} 23$ & $\mathrm{Cl} 3$ & $117.7(7)$ \\
\hline N1 & $\mathrm{C} 5$ & $\mathrm{C} 4$ & $111.0(5)$ & $\mathrm{Cl1}$ & $\mathrm{C} 23$ & $\mathrm{Cl} 2$ & $113.4(6)$ \\
\hline C6 & $\mathrm{C} 5$ & $\mathrm{C} 4$ & $109.2(5)$ & $\mathrm{Cl} 3$ & $\mathrm{C} 23$ & $\mathrm{Cl} 2$ & $106.2(5)$ \\
\hline C9 & $\mathrm{C} 8$ & $\mathrm{C} 7$ & $120.7(8)$ & $\mathrm{C} 3$ & $\mathrm{C} 2$ & $\mathrm{C} 1$ & $126.0(9)$ \\
\hline $\mathrm{C} 17$ & $\mathrm{C} 18$ & $\mathrm{C} 22$ & $115.1(7)$ & $\mathrm{C} 2$ & $\mathrm{C} 3$ & $\mathrm{C} 4$ & $121.0(8)$ \\
\hline C17 & $\mathrm{C} 18$ & C19 & $103.3(6)$ & & & & \\
\hline
\end{tabular}

Table 6 Torsion Angles for ZJ-04-57.

\begin{tabular}{|c|c|c|c|c|c|}
\hline $\mathbf{A}$ & $\mathbf{C}$ & D & Angle $/^{\circ}$ & A $\mathbf{B}$ & Angle $/^{\circ}$ \\
\hline $\mathrm{O} 2 \mathrm{~S} 1$ & $\mathrm{~N} 2$ & C6 & $-52.2(5)$ & C13C18C19C15 & $51.2(5)$ \\
\hline $\mathrm{O} 3 \mathrm{~S} 1$ & $\mathrm{~N} 2$ & C6 & $77.7(5)$ & $\mathrm{C} 17 \mathrm{C} 18 \mathrm{C} 19 \mathrm{C} 20$ & $-179.6(7)$ \\
\hline C22 S1 & $\mathrm{N} 2$ & C6 & $-166.5(5)$ & $\mathrm{C} 22 \mathrm{C} 18 \mathrm{C} 19 \mathrm{C} 20$ & $51.7(8)$ \\
\hline $\mathrm{O} 2 \mathrm{~S} 1$ & $\mathrm{~N} 2$ & $\mathrm{C} 13$ & $104.3(5)$ & C13 C18C19C20 & $-69.3(7)$ \\
\hline $\mathrm{O} 3 \mathrm{~S} 1$ & $\mathrm{~N} 2$ & $\mathrm{C} 13$ & $-125.8(4)$ & C7 N1 C1 C2 & $175.8(6)$ \\
\hline C22 S1 & $\mathrm{N} 2$ & C13 & $-10.0(5)$ & C5 N1 C1 C2 & $22.9(8)$ \\
\hline C6 N2 & $\mathrm{C} 1$ & C14 & $-62.2(7)$ & N2 C13C14C15 & $-127.9(6)$ \\
\hline $\mathrm{S} 1 \mathrm{~N} 2$ & $\mathrm{C} 1$ & $\mathrm{C} 14$ & $140.3(5)$ & C18C13C14C15 & $-10.8(7)$ \\
\hline $\mathrm{C} 6 \mathrm{~N} 2$ & $\mathrm{C} 1$ & C18 & $-178.1(4)$ & $\mathrm{C} 12 \mathrm{C} 11 \mathrm{C} 10 \mathrm{C} 9$ & $0.5(13)$ \\
\hline $\mathrm{S} 1 \mathrm{~N} 2$ & $\mathrm{C} 1$ & C18 & $24.4(6)$ & $\mathrm{C} 22 \mathrm{C} 18 \mathrm{C} 17 \mathrm{C} 16$ & $172.1(6)$ \\
\hline C5 N1 & $\mathrm{C} 7$ & $\mathrm{C} 12$ & $-1.6(8)$ & C19C18C17C16 & $41.2(8)$ \\
\hline $\mathrm{C} 1 \mathrm{~N} 1$ & $\mathrm{C} 7$ & $\mathrm{C} 12$ & $-154.4(6)$ & $\mathrm{C} 13 \mathrm{C} 18 \mathrm{C} 17 \mathrm{C} 16$ & $-67.0(7)$ \\
\hline C5 N1 & $\mathrm{C} 7$ & $\mathrm{C} 8$ & $-178.9(5)$ & $\mathrm{C} 11 \mathrm{C} 10 \mathrm{C} 9 \mathrm{C} 8$ & $-0.4(13)$ \\
\hline C1 N1 & $\mathrm{C} 7$ & $\mathrm{C} 8$ & $28.3(8)$ & C7 C8 C9 C10 & $0.5(12)$ \\
\hline $\mathrm{C} 13 \mathrm{~N} 2$ & C6 & $\mathrm{O} 1$ & $4.7(8)$ & N1 C5 C4 C3 & $44.4(8)$ \\
\hline $\mathrm{S} 1 \mathrm{~N} 2$ & C6 & $\mathrm{O} 1$ & $159.7(4)$ & C6 C5 C4 C3 & $-79.8(7)$ \\
\hline $\mathrm{C} 13 \mathrm{~N} 2$ & C6 & $\mathrm{C} 5$ & $-178.4(5)$ & C21 C19C15C14 & $-175.2(7)$ \\
\hline $\mathrm{S} 1 \mathrm{~N} 2$ & C6 & C5 & $-23.4(7)$ & C20C19C15C14 & $61.4(8)$ \\
\hline C7 N1 & $\mathrm{C} 5$ & C6 & $-75.6(7)$ & C18C19C15C14 & $-58.4(7)$ \\
\hline $\mathrm{C} 1 \mathrm{~N} 1$ & $\mathrm{C} 5$ & C6 & $76.9(6)$ & C21 C19C15C16 & $-64.4(8)$ \\
\hline C7 N1 & $\mathrm{C} 5$ & $\mathrm{C} 4$ & $161.9(5)$ & C20C19C15C16 & $172.1(6)$ \\
\hline C1 N1 & C5 & $\mathrm{C} 4$ & $-45.7(7)$ & C18C19C15C16 & $52.3(6)$ \\
\hline
\end{tabular}




\begin{tabular}{|c|c|c|}
\hline O1 C6 C5 N1 & $-21.6(8)$ C13C14C15C19 & $45.0(8)$ \\
\hline N2 C6 C5 N1 & 161.6(5) C13 C14C15C16 & $-61.0(8)$ \\
\hline $\mathrm{O} 1 \mathrm{C} 6 \mathrm{C} 5 \mathrm{C} 4$ & 102.0(6) C18C17C16C15 & $-6.2(8)$ \\
\hline $\mathrm{N} 2 \mathrm{C} 6 \mathrm{C} 5 \mathrm{C} 4$ & $-74.9(6) \mathrm{C} 19 \mathrm{C} 15 \mathrm{C} 16 \mathrm{C} 17$ & $-30.8(8)$ \\
\hline $\mathrm{C} 12 \mathrm{C} 7 \mathrm{C} 8 \mathrm{C} 9$ & $-0.6(10) \mathrm{C} 14 \mathrm{C} 15 \mathrm{C} 16 \mathrm{C} 17$ & $75.1(9)$ \\
\hline N1 $\mathrm{C} 7 \quad \mathrm{C} 8$ C9 & 176.9(7) $\mathrm{C} 10 \mathrm{C} 11 \mathrm{C} 12 \mathrm{C} 7$ & $-0.6(12)$ \\
\hline N2 C13C18C17 & $-154.6(6)$ N1 C7 C12C11 & $-176.8(7)$ \\
\hline $\mathrm{C} 14 \mathrm{C} 13 \mathrm{C} 18 \mathrm{C} 17$ & 82.2(7) C8 C7 C12C11 & $0.6(10)$ \\
\hline $\mathrm{N} 2 \mathrm{C} 13 \mathrm{C} 18 \mathrm{C} 22$ & $-30.0(6) \mathrm{C} 17 \mathrm{C} 18 \mathrm{C} 22 \mathrm{~S} 1$ & $142.3(5)$ \\
\hline $\mathrm{C} 14 \mathrm{C} 13 \mathrm{C} 18 \mathrm{C} 22$ & $-153.2(6) \mathrm{C} 19 \mathrm{C} 18 \mathrm{C} 22 \mathrm{~S} 1$ & $-94.7(6)$ \\
\hline N2 $\mathrm{C} 13 \mathrm{C} 18 \mathrm{C} 19$ & 97.1(5) C13 C18C22 S1 & $23.1(7)$ \\
\hline C14C13C18C19 & $-26.1(6) \mathrm{O} 2 \mathrm{~S} 1 \mathrm{C} 22 \mathrm{C} 18$ & $-121.5(5)$ \\
\hline C17C18C19C21 & 57.3(8) O3 S1 C22C18 & $105.1(6)$ \\
\hline C22 C18C19C21 & $-71.4(7)$ N2 S1 C22C18 & $-8.2(6)$ \\
\hline C13C18C19C21 & 167.7(6) N1 C1 C2 C3 & $2.6(13)$ \\
\hline $\mathrm{C} 17 \mathrm{C} 18 \mathrm{C} 19 \mathrm{C} 15$ & $-59.2(7) \mathrm{C} 1 \quad \mathrm{C} 2 \quad \mathrm{C} 3 \mathrm{C} 4$ & $-1.5(16)$ \\
\hline $\mathrm{C} 22 \mathrm{C} 18 \mathrm{C} 19 \mathrm{C} 15$ & 172.1(5) $\mathrm{C} 5 \mathrm{C} 4 \mathrm{C} 3 \mathrm{C} 2$ & $-22.5(12)$ \\
\hline
\end{tabular}

Table 7 Hydrogen Atom Coordinates $\left(\AA \times 10^{4}\right)$ and Isotropic Displacement Parameters $\left(\AA^{2} \times 10^{3}\right)$ for $\mathrm{ZJ-04-57.}$

$\begin{array}{lrrrr}\text { Atom } & \boldsymbol{x} & \boldsymbol{y} & \boldsymbol{z} & \mathbf{U}(\mathbf{e q}) \\ \text { H13 } & 9499 & 9196 & 2763 & 66 \\ \text { H11 } & 13358 & 8364 & 1124 & 100 \\ \text { H5 } & 13303 & 8414 & 3149 & 65 \\ \text { H8 } & 15465 & 11236 & 2247 & 87 \\ \text { H1A } & 11787 & 3067 & 88 \\ \text { H1B } & 13795 & 11397 & 3213 & 88 \\ \text { H14A } & 15157 & 10715 & 3348 & 99 \\ \text { H14B } & 8901 & 10266 & 3936 & 99 \\ \text { H10 } & 9680 & 9617 & 614 & 110 \\ \text { H17A } & 14761 & 7252 & 3096 & 132 \\ \text { H17B } & 7183 & 8068 & 2586 & 132 \\ \text { H9 } & 7847 & 11011 & 1180 & 104 \\ \text { H4A } & 15796 & 8816 & 4251 & 93 \\ \text { H4B } & 12746 & 8679 & 4116 & 113 \\ \text { H15 } & 14164 & 9891 & 4223 & 117 \\ \text { H20A } & 7522 & 7431 & 4655 & 117 \\ \text { H20B } & 9780 & 8521 & 4988 & 117 \\ \text { H20C } & 9140 & 8741 & 4457 & 147 \\ \text { H21A } & 10125 & 7375 & 4064 & 147 \\ \text { H21B } & 6733 & 7625 & 4758 & 147 \\ \text { H21C } & 7176 & 6566 & 4371 & \end{array}$




$\begin{array}{lrrrr}\text { H16A } & 6246 & 8890 & 3470 & 155 \\ \text { H16B } & 7026 & 9716 & 3015 & 155 \\ \text { H12 } & 13005 & 8563 & 2186 & 87 \\ \text { H22A } & 9428 & 6425 & 2900 & 98 \\ \text { H22B } & 9473 & 6206 & 3635 & 98 \\ \text { H23 } & 11164 & 12214 & 3731 & 126 \\ \text { H2 } & 14137 & 11978 & 4169 & 118 \\ \text { H3 } & 13546 & 10489 & 4745 & 122\end{array}$

